\title{
UNDERSTANDING THE INTRINSIC WATER WETTABILITY OF GRAPHITE
}

by

Andrew Kozbial

B.S. in Chemical Engineering, University of Toledo, 2010

Submitted to the Graduate Faculty of

The Swanson School of Engineering in partial fulfillment

of the requirements for the degree of

Doctor of Philosophy in Chemical Engineering

University of Pittsburgh 
UNIVERSITY OF PITTSBURGH

SWANSON SCHOOL OF ENGINEERING

This dissertation was presented

by

Andrew Kozbial

It was defended on

March 31, 2016

and approved by

Sachin Velankar, Ph.D., Associate Professor

Department of Chemical \& Petroleum Engineering

Giannis Mpourmpakis, Ph.D., Assistant Professor

Department of Chemical \& Petroleum Engineering

Haitao Liu, Ph.D., Assistant Professor

Department of Chemistry

Dissertation Director: Lei Li, Ph.D., Assistant Professor

Department of Chemical \& Petroleum Engineering 
Copyright (C) by Andrew Kozbial 2016 


\title{
UNDERSTANDING THE INTRINSIC WATER WETTABILITY OF GRAPHITE
}

\author{
Andrew Kozbial, Ph.D.
}

University of Pittsburgh, 2016

Decades of research since the 1940s has substantiated graphite as a low surface energy material. Its chemical structure led researchers to believe that airborne hydrocarbon contamination was inconsequential and contradictory reports were not convincing. Graphite gained renewed interest when graphene was first isolated in 2004. Being an atomically thin material, the surface properties of graphene are critical to its performance, thus elucidating surface properties of graphene and graphite became important topics in fundamental and applied research.

This work began with the realization that fresh graphene and graphite are mildly hydrophilic and approach their established hydrophobicity upon exposure to ambient air. Hydrocarbons in ambient air adsorb onto the fresh surface and cause it to appear hydrophobic. This work was first published in 2013 (doi: 10.1038/nmat3709) and provided the basis for further exploration of the intrinsic chemical nature of graphene, graphite, and $\mathrm{MoS}_{2}$.

Fresh graphite is shown to be mildly hydrophilic and becomes hydrophobic upon exposure to ambient air. Similar behaviour was observed for graphene and $\mathrm{MoS}_{2}$. Ellipsometry showed growth of an adsorptive layer on the fresh (clean) surface and ATR-FTIR indicated that the adsorptive layer was airborne hydrocarbon. Theoretical calculation further confirmed that adsorption of only a monolayer of hydrocarbon is enough to reproduce the hydrophobic behavior previously observed on HOPG. 
Surface energy of fresh CVD graphene was calculated to be $62.2 \pm 3.1 \mathrm{~mJ} / \mathrm{m}^{2}$ (Fowkes), $53.0 \pm 4.3 \mathrm{~mJ} / \mathrm{m}^{2}$ (Owens-Wendt), and $63.8 \pm 2.0 \mathrm{~mJ} / \mathrm{m}^{2}$ (Neumann), which decreased to $45.6 \pm$ $3.9 \mathrm{~mJ} / \mathrm{m}^{2}, 37.5 \pm 2.3 \mathrm{~mJ} / \mathrm{m}^{2}$, and $57.4 \pm 2.1 \mathrm{~mJ} / \mathrm{m}^{2}$, respectively, after 24 hours of air exposure. Similar behaviour also occurred for HOPG and $\mathrm{MoS}_{2}$. The fresh surface exhibits highest surface energy which decreases upon adsorption of airborne hydrocarbons. Results also indicate that the fresh surface is mildly polar.

Analysis based on defect density and dynamic contact angle measurements determine that the intrinsic WCA of fresh $\mathrm{sp}^{2}$-hybridized carbon is $70.0^{\circ} \pm 1.5^{\circ}$. Current understanding of wetting models show that roughness and chemical heterogeneity do not cause the intrinsic hydrophilicity. This work unequivocally shows that fresh graphitic surfaces are mildly hydrophilic. 
TABLE OF CONTENTS

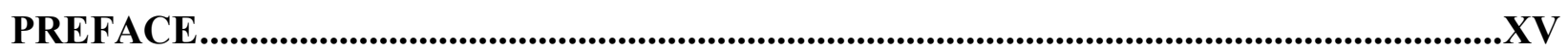

1.0 INTRODUCTION …................................................................................................................. 1

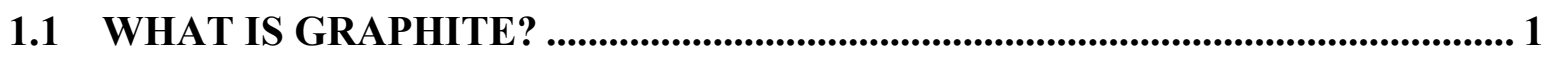

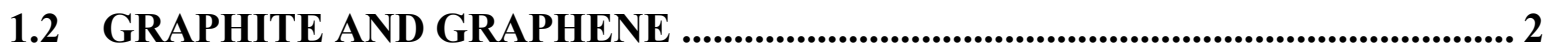

1.3 IMPLICATIONS OF SURFACE CONTAMINATION.......................................... 4

2.0 UNDERSTANDING THE INTRINSIC WATER WETTABILITY OF GRAPHITE 10

2.1 HISTORY OF GRAPHITE HYDROPHOBICITY ….............................................. 10

2.2 TIME EVOLUTION OF WCA AND HYDROCARBON ADSORPTION .......... 12

2.3 REMOVAL OF HYDROCARBON CONTAMINANTS ......................................... 15

2.4 INTRINSIC WCA OF GRAPHITE ................................................................... 18

2.4.1 Theoretical calculation ........................................................................................ 19

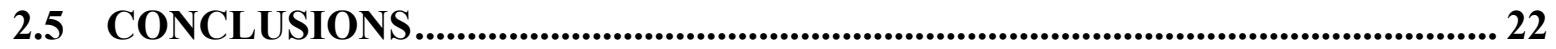

3.0 DETERMINING THE SURFACE ENERGY OF GRAPHENE ................................... 24

3.1 HISTORY OF GRAPHENE HYDROPHOBICITY................................................ 24

3.2 SURFACE FREE ENERGY MODELS ...................................................................... 27

3.2.1 Equation of state................................................................................................. 29

3.2.1.1 Neumann model ........................................................................................... 31

3.2.2 Dissociation into components ............................................................................. 32 
3.2.2.1 Fowkes model .............................................................................................. 33

3.2.2.2 Owens-Wendt model .............................................................................. 34

3.2.3 Remarks on the surface energy models.......................................................... 35

3.3 GRAPHENE CHARACTERIZATION ...............................................................35

3.4 CONTACT ANGLE AND SURFACE ENERGY ...............................................37

3.5 ELLIPSOMETRY CHARACTERIZATION .............................................................. 43

3.6 EFFECT OF SURFACE ROUGHNESS............................................................... 44

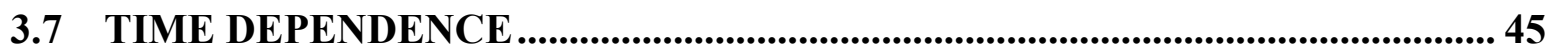

3.8 ORIGIN OF INTRINSIC POLARITY …...................................................................... 47

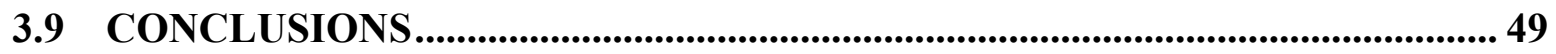

4.0 QUANTIFYING THE DEFECT DENSITY OF GRAPHITE ...................................... 51

4.1 RAMAN SPECTROSCOPY ..................................................................................... 51

4.1.1 Raman data acquisition and analysis ................................................................ 54

4.1.2 Surface sensitivity …...........................................................................................56

4.1.3 Raman spectra peak fitting .................................................................................. 58

4.1.4 Calculating defect density .................................................................................... 60

4.1.5 Number of defects in WCA experiments .......................................................... 66

4.2 AFM: SURFACE TOPOGRAPHY …........................................................................... 68

4.2.1 Step edge density ..................................................................................................... 69

4.2.2 Defects caused by exfoliation .......................................................................... 71

4.2.3 Number of defects to carbon atoms ..................................................................... 73

5.0 INTRINSIC WATER WETTABILITY OF GRAPHITE ................................................. 75

5.1 STATIC WATER CONTACT ANGLE .................................................................. 75 
5.2 DYNAMIC WCA AND HYSTERESIS....................................................................... 80

5.2.1 Theory and background of hysteresis ...................................................... 80

5.2.2 Current understanding of static and dynamic contact angles ................. 87

5.2.3 Dynamic WCA on graphite ..................................................................... 91

5.2.4 Calculating surface free energy from hysteresis .................................. 96

5.3 WENZEL AND CASSIE-BAXTER WETTING MODELS..............................98

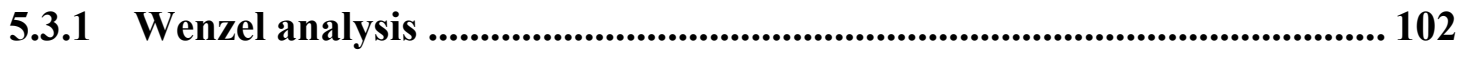

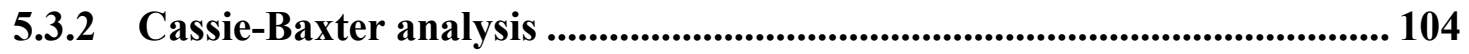

5.3.2.1 Comments on Cassie-Baxter analysis .......................................... 109

5.3.3 Israelachvili equation............................................................................ 110

5.3.4 Line energy of the triple phase contact line ......................................... 112

5.4 HOW METHOD OF EXFOLIATION AFFECTS WETTABILITY ................ 114

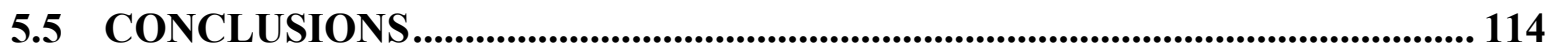

6.0 UNDERSTANDING THE INTRINSIC WATER WETTABILITY OF MoS $2 \ldots . . . . . .116$

6.1 HISTORY OF 2D MATERIAL WETTABILITY ..................................... 116

6.2 WATER WETTABILITY AND SURFACE ENERGY ................................... 119

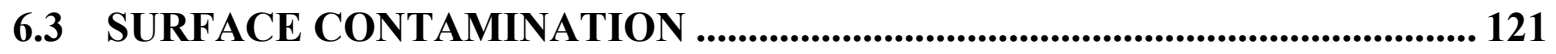

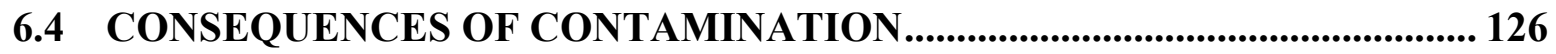

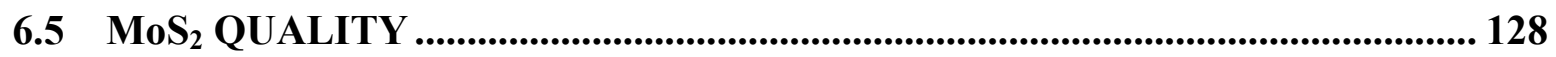

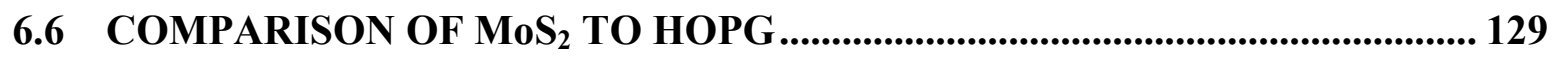

6.7 CONCLUSIONS ............................................................................................................. 130

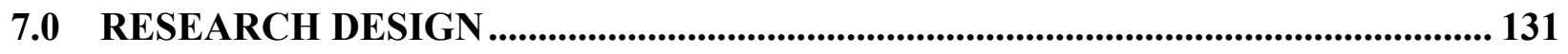

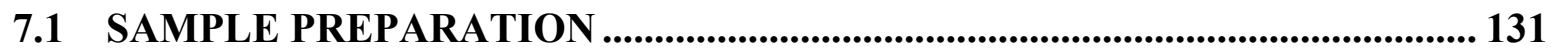


7.1.1 HOPG and pyrolytic graphite........................................................................... 131

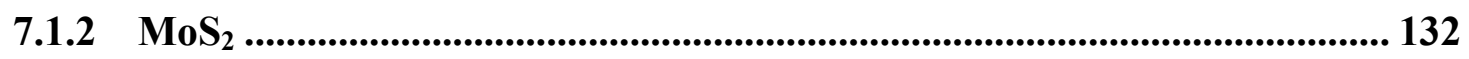

7.1.3 Synthesis of CVD graphene.............................................................................. 132

7.2 WATER CONTACT ANGLE (WCA) .................................................................. 133

7.2.1 Graphene................................................................................................................. 133

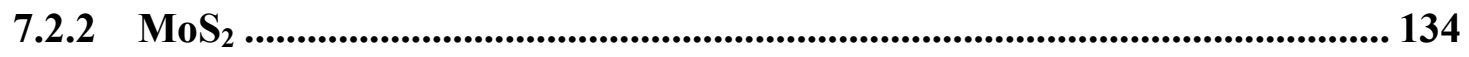

7.2.3 Saturated 1-octadecene vapor bath for HOPG ............................................... 134

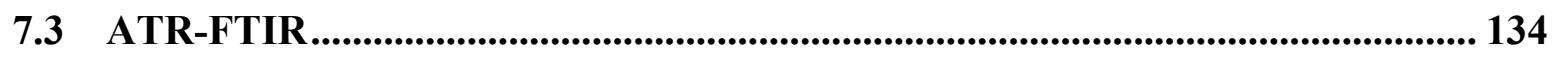

7.4 SPECTROSCOPIC ELLIPSOMETRY ....................................................................... 135

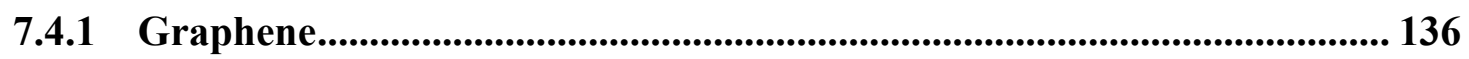

7.5 RAMAN SPECTROSCOPY ................................................................................. 136

7.6 X-RAY PHOTOELECTRON SPECTROSCOPY (XPS)....................................... 137

7.7 ATOMIC FORCE MICROSCOPY (AFM) ............................................................. 137

$7.8 \quad$ X-RAY DIFFRACTION (XRD) ............................................................................. 138

7.9 ULTRA HIGH VACUUM (UHV) TREATMENT OF HOPG ............................. 138

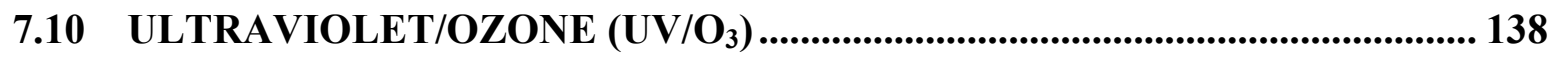

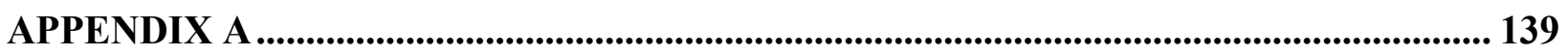

DETAILS OF ELLIPSOMETRY DATA ANALYSIS .................................................... 139

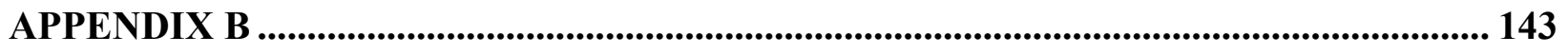

ELUCIDATING THE CRYSTAL STRUCTURE OF GRAPHITE ............................. 143

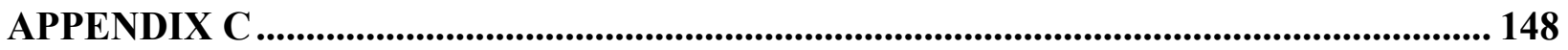

ELUCIDATING SURFACE CHEMISTRY BY XPS....................................................... 148

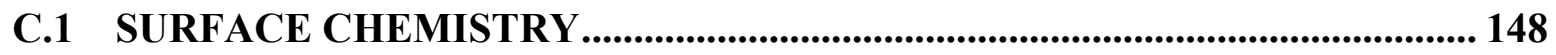


C.2 C1s PEAK FITTING METHODOLOGY ............................................................... 154

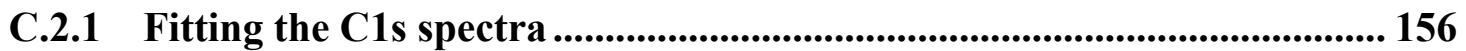

C.3 ANGLE RESOLVED XPS (AR-XPS) ......................................................... 160

C.4 ATTENUATION AND EFFECTS OF CONTAMINATION ............................. 165

C.5 XPS CONCLUSIONS ................................................................................. 167

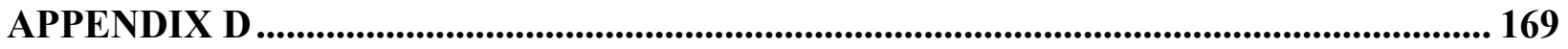

DETAILS OF XPS DATA ACQUISITION AND ANALYSIS .................................... 169

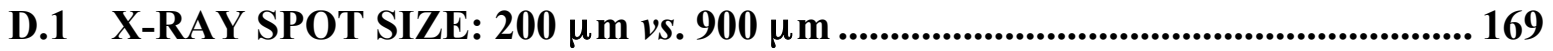

D.2 C1s FITTING PROCEDURE............................................................................... 170

D.3 COMMENTS ON ERROR OF C1s FIT ................................................................ 177

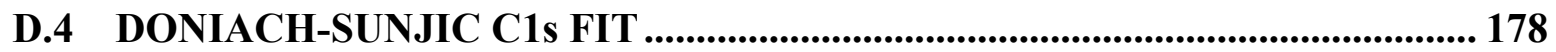

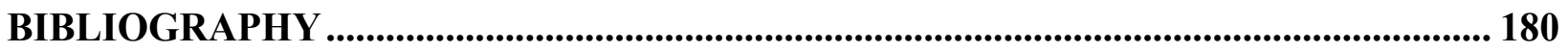




\section{LIST OF TABLES}

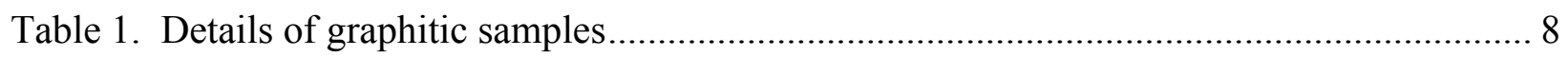

Table 2. Polar and dispersive surface energy components of test liquids ................................... 27

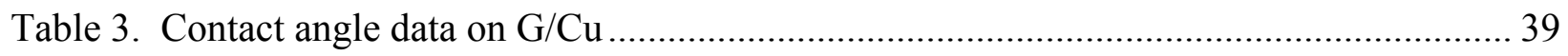

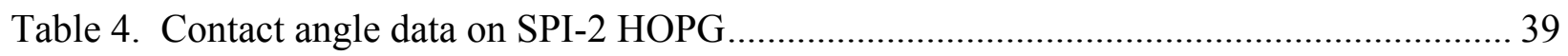

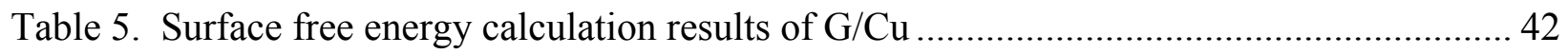

Table 6. Surface free energy calculation results of SPI-2 HOPG ............................................... 42

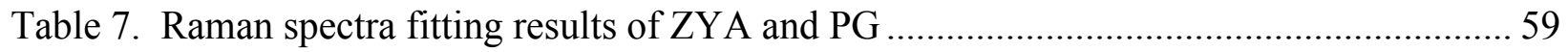

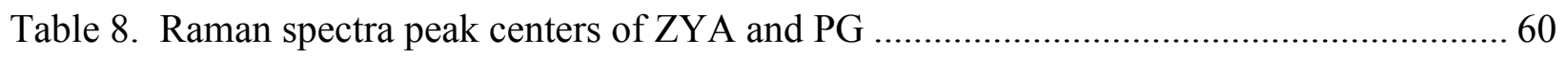

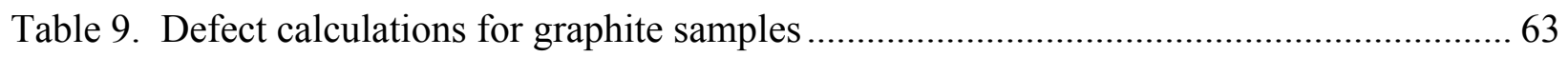

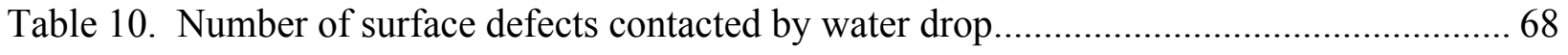

Table 11. Static WCA of fresh graphitic surface exfoliated with tape and razor ........................ 78

Table 12. Literature data on the intrinsic hydrophilicity of graphite .......................................... 79

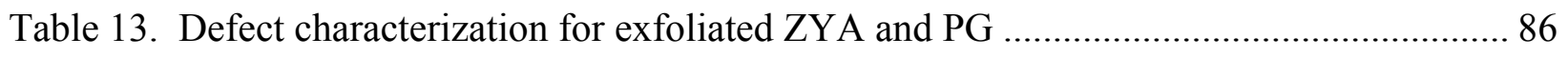

Table 14. Dynamic WCA and hysteresis of graphite samples................................................... 93

Table 15. Surface energy of fresh graphite using various models ............................................... 97

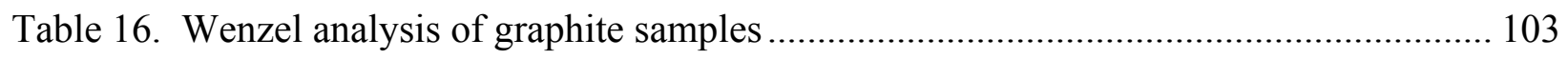

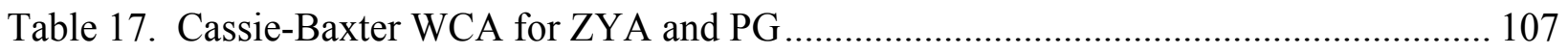

Table 18. Cassie-Baxter and Israelachvili wetting model results ............................................ 111 
Table 19. Equilibrium WCA using the Tadmor equation......................................................... 113

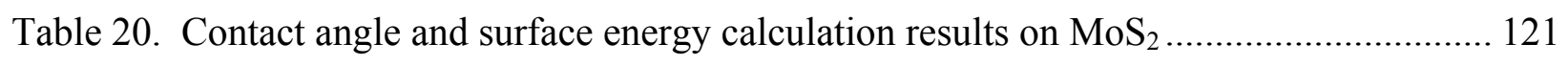

Table 21. Index of refraction (n) and extinction coefficient (k) of SPI-2 HOPG....................... 141

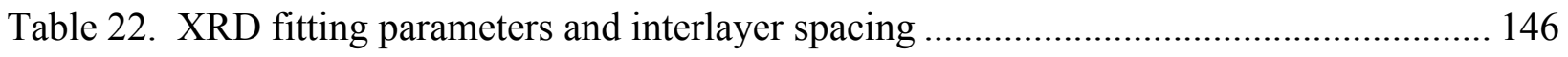

Table 23. Peak attribution and fitting results for the C1s spectra of ZYA and PG .................. 158

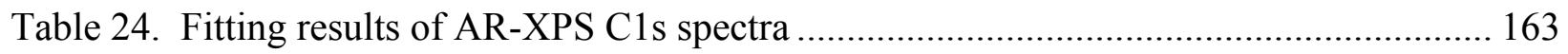

Table 25. Contribution of surface layer to XPS spectrum ...................................................... 166

Table 26. XPS fitting results of ZYA C1s spectra for different x-ray spot sizes ...................... 170

Table 27. Details of functions used for fitting the C1s spectrum ........................................... 173

Table 28. Parameters of functions used for fitting the C1s spectrum ....................................... 174

Table 29. Observations and notes of peak fitting models....................................................... 178 


\section{LIST OF FIGURES}

Figure 1. Optical and AFM images of the graphite samples ............................................ 9

Figure 2. WCA and ATR-FTIR of fresh and aged HOPG ............................................... 13

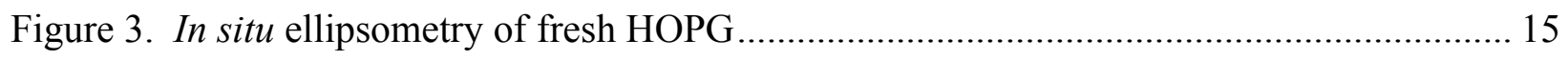

Figure 4. Removal of hydrocarbon contaminants........................................................ 17

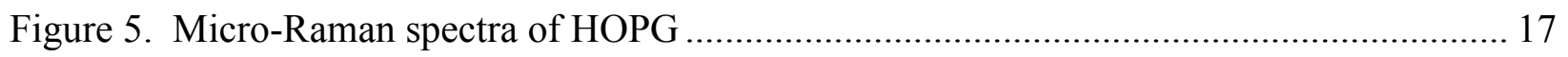

Figure 6. Theoretical model of WCA on polyethylene (PE) ............................................ 22

Figure 7. Schematic depicting the interfaces of a liquid drop on a solid surface ..................... 26

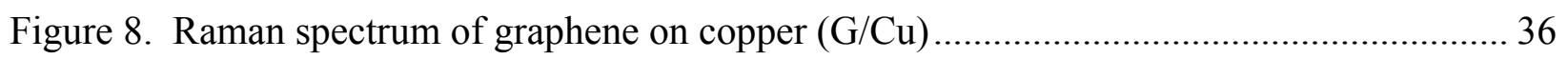

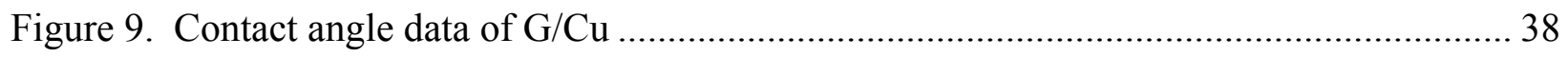

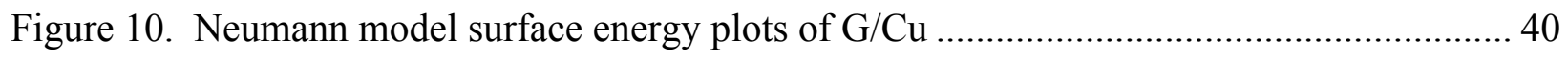

Figure 11. Owens-Wendt (extended Fowkes) surface energy plots of $\mathrm{G} / \mathrm{Cu}$........................ 41

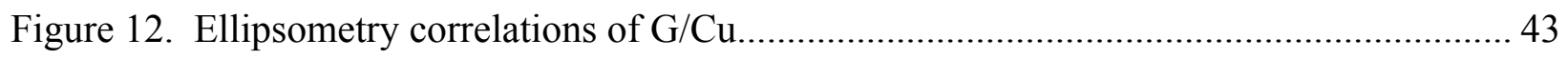

Figure 13. Point defects that occur in a crystal lattice ................................................... 53

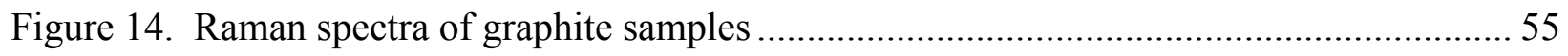

Figure 15. AFM height and phase images of ZYA, PG, SPI-1, and SPI-2 .......................... 70

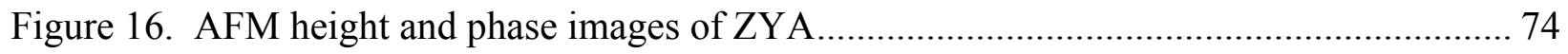

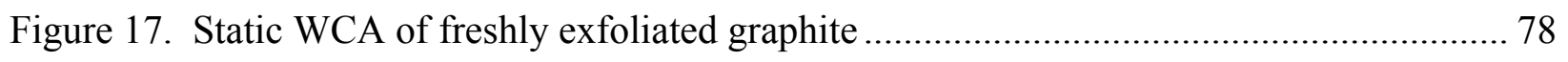

Figure 18. Schematic of contact line pinning for a water drop on a hydrophilic surface........... 83

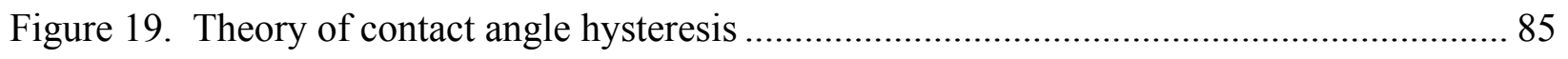

Figure 20. Advancing contact angle mechanism ............................................................. 89 
Figure 21. Receding contact angle mechanism....................................................................... 90

Figure 22. Dynamic WCA of freshly exfoliated graphite......................................................... 92

Figure 23. Hysteresis of freshly exfoliated graphite................................................................. 92

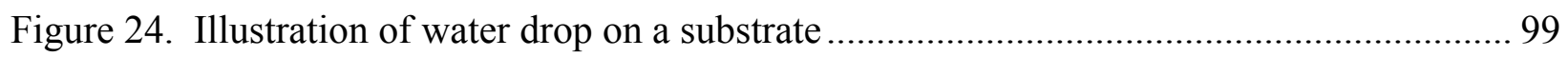

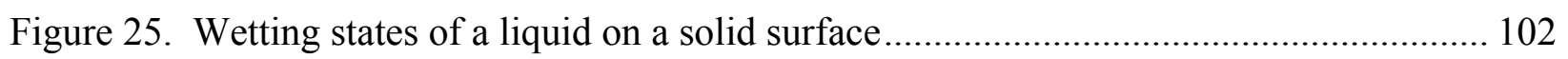

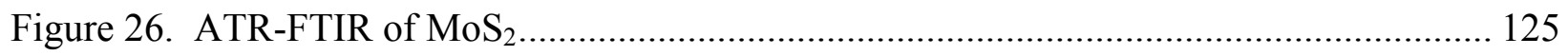

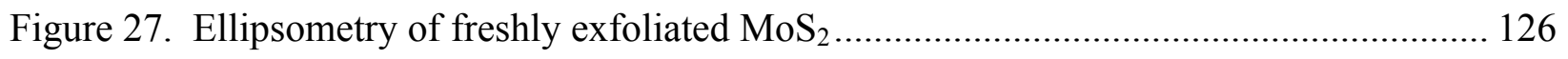

Figure 28. Correlation between surface contamination and WCA/surface energy ................... 128

Figure 29. Temporal monitoring of ellipsometric quantities .................................................. 141

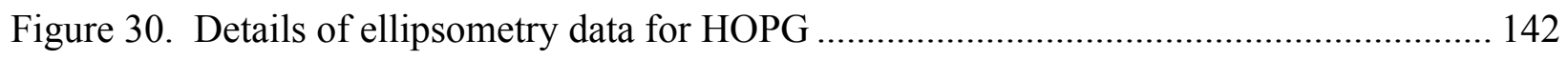

Figure 31. XRD spectra of freshly exfoliated graphite......................................................... 147

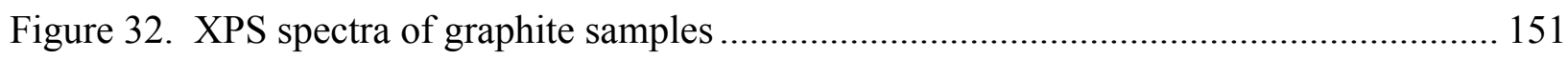

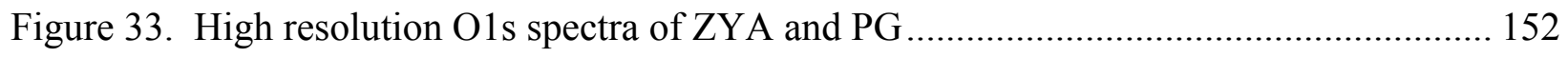

Figure 34. C KLL Auger spectra of graphite samples ............................................................ 153

Figure 35. High resolution C1s spectra of ZYA and PG ..................................................... 159

Figure 36. High resolution C1s spectra where only peaks C1-C3 are fit ................................ 160

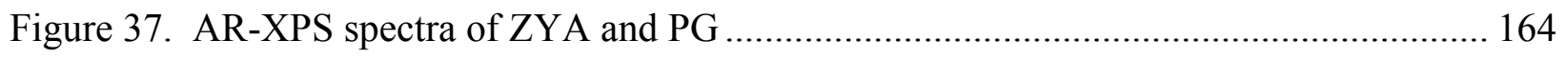

Figure 38. High resolution C1s spectra for different x-ray spot sizes ..................................... 172

Figure 39. Fitting results of C1s spectra by various models................................................. 175

Figure 40. Peak fitting the C1s spectra using various models .................................................. 176

Figure 41. Doniach-Sunjic fitting results of the C1s spectrum................................................ 179 


\section{PREFACE}

\section{Acknowledgements}

I would like to thank my dissertation advisor, Dr. Lei Li, for his support, patience, and guidence during the past five years. My time at Pitt has been very rewearding both personally and professionally and Lei has had a tremendous impact on my success. I would also like to thank Dr. Haitao Liu for his support and guidance over the past few years as well. Our collaborative efforts have been very successful and I am fortunate to have had the opportunity to work with him and several of his students on various projects. I would also like to thank Zhiting Li, Yongjin Wang, Mo Salim, Vahid Vahdat, and Xiao Gong who I have worked closely with on several projects. Our enriching discussions have enabled me to become a better scientist. Additionally, I have been fortunate to work with many wonderful undergraduate students who have unknowingly taught me how to educate younger scientists. Their efforts in the lab are greatly appreciated. Additionally, thanks to Dr. Velankar and Dr. Mpourmpakis for serving on my committee. The past five years have certainly been a collaborative effort of many people who have substantially helped me along the way. 


\section{Remarks}

Throughout my dissertation are footnotes which briefly describe some of the scientists behind the theory and models used in my work. I am very curious about the history of science and learning about the real people who have developed these concepts that scientsts either still use and debate - today or have become the foundation of newer scientific concepts. My interest in the history of science first piqued when listening to a story about Fritz Haber on NPR while driving home to Ohio one afternoon a few years ago. ${ }^{*}$ This made me realize that past scientists were not just people who worked tirelessly in a lab but were also interesting people whose livlihoods were often caught up in politics and wars of their time.

Particularly interesting was the conncection I discovered of Robert Wenzel to the Mellon Institute of Industrial Research. The Mellon Institute is now part of Carnegie Mellon University and is located adjacent to the main campus of the University of Pittsburgh, only a few blocks away from where I have worked the past five years. I find captivating that scientists worked nearly a century ago where I and thousands of other students learn and work today. While Dr. Wenzel performed neither his original work nor formulated the Wenzel model at the current Mellon Institue building (the current building was built in 1937 and Dr. Wenzel's paper was published in 1936), he did work in the original Mellon Institute building, Allen Hall, which is directly across the street from Benedum Hall, the engineering building at the University of Pittsburgh. So cool! Today, Allen Hall is home to Pitt's Department of Physics and Astronomy.

Eventhough most of the scientists we hear of today are ambiguous figures that most people don't care about, I find it important for current and future scientists to be familiar with the

\footnotetext{
* How Do You Solve a Problem Like Fritz Haber? http://www.radiolab.org/story/180132-how-do-yousolve-problem-fritz-haber/ (accessed March 1, 2016).
} 
history of science and the story behind the concepts which we learn today. At the very least, it's important for students to realize that past scientists were actual people. Much of the foundation of scientific knowledge was researched during times of conflict and war such as European conflicts during the 1700 s and 1800 s and the World Wars of the 1900s. This leads to very interesting stories about the struggles of the scientists and the causes for their work, such as the story of Fritz Haber. Therefore, as my dissertation is a medium to disseminate my own research over the past five years, I would like to include a few notes about previous researchers that I find interesting. 


\section{Nomenclature}

\begin{tabular}{|c|c|c|c|}
\hline AFM & atomic force microscopy & PAH & $\begin{array}{l}\text { polycyclic aromatic } \\
\text { hydrocarbons }\end{array}$ \\
\hline AR-XPS & angle resolved-XPS & PG & $\begin{array}{l}\text { pyrolytic graphite; } \\
\text { pyrolytic carbon }\end{array}$ \\
\hline ATR-FTIR & $\begin{array}{l}\text { attenuated total reflectance-Fourier } \\
\text { transform infrared spectroscopy }\end{array}$ & $\mathrm{r}$ & Wenzel roughness parameter \\
\hline at $\%$ & atomic percent & $\mathrm{R}_{\mathrm{a}}$ & surface roughness \\
\hline CVD & chemical vapour deposition & $r_{d}$ & defect radius \\
\hline $\mathrm{D}$ & diameter of defects & REO & rare earth oxide \\
\hline DCA & diiodomethane contact angle & SEM & scanning electron microscopy \\
\hline $\mathrm{E}_{\mathrm{L}}$ & Raman laser energy & sf & area fraction of defects \\
\hline $\mathrm{f}$ & $\begin{array}{l}\text { fraction of defects along } \\
\text { the contact line }\end{array}$ & $\mathrm{SiO}_{2}$ & silicon dioxide \\
\hline $\mathrm{f}_{1}$ & fraction of defects & SPI-1 & $\begin{array}{l}\text { high grade HOPG } \\
\text { (ZYA grade) }\end{array}$ \\
\hline $\mathrm{f}_{2}$ & fraction of basal plane & SPI-2 & $\begin{array}{l}\text { intermediate grade } \mathrm{HOPG} \\
\text { (ZYB grade) }\end{array}$ \\
\hline FWHM & full width at half maximum & TMDC & transition metal dichalcogenide \\
\hline $\mathrm{G} / \mathrm{Cu}$ & graphene/copper & UHV & ultra-high vacuum \\
\hline $\mathrm{G} / \mathrm{Ni}$ & graphene/nickel & $\mathrm{W}$ & interfacial work of adhesion \\
\hline HOPG & highly ordered pyrolytic graphite & WCA & water contact angle \\
\hline $\mathrm{I}_{\mathrm{D}}$ & intensity of the Raman D peak & $\mathrm{WCA}_{\mathrm{a}}$ & advancing WCA \\
\hline $\mathrm{I}_{\mathrm{G}}$ & intensity of the Raman G peak & $\mathrm{WCA}_{\mathrm{r}}$ & receding $\mathrm{WCA}$ \\
\hline
\end{tabular}




\begin{tabular}{|c|c|c|c|}
\hline $\mathrm{I}_{\mathrm{S}}$ & $\begin{array}{l}\text { intensity of XPS signal due } \\
\text { to surface layer }\end{array}$ & $\mathrm{x}_{\mathrm{c}}$ & peak center \\
\hline $\mathrm{L}$ & pitch of defects & XPS & $\begin{array}{l}\text { x-ray photoelectron } \\
\text { spectroscopy }\end{array}$ \\
\hline $\mathrm{L}_{\mathrm{a}}$ & crystallite domain size & $\mathrm{XRD}$ & $\mathrm{x}$-ray diffraction \\
\hline $\mathrm{L}_{\mathrm{d}}$ & distance between defects & ZYA & high grade HOPG \\
\hline $\mathrm{L}_{\mathrm{L}}$ & Raman laser spot size & ZYB & intermediate grade HOPG \\
\hline $\mathrm{MoS}_{2}$ & molybdenum disulfide & $\mathrm{ZYH}$ & low grade HOPG \\
\hline $\mathrm{n}_{\mathrm{d}}$ & defect density & & \\
\hline$\gamma$ & $\begin{array}{l}\text { liquid surface tension; } \\
\text { solid surface free energy }\end{array}$ & $\theta$ & contact angle $\left(\right.$ degrees; $\left.{ }^{\circ}\right)$ \\
\hline$\gamma_{1}$ & liquid surface tension & $\theta_{\mathrm{a}}$ & advancing contact angle \\
\hline$\gamma_{\mathrm{lv}}$ & $\begin{array}{l}\text { liquid-vapour surface tension } \\
\text { (equivalent to } \gamma_{1} \text { ) }\end{array}$ & $\theta_{\mathrm{CB}}$ & Cassie-Baxter contact angle \\
\hline$\gamma_{\mathrm{s}}$ & solid surface energy & $\theta_{\mathrm{H}}$ & hysteresis \\
\hline$\gamma_{\mathrm{s}}^{\mathrm{d}}$ & $\begin{array}{l}\text { dispersive component of } \\
\text { solid surface energy }\end{array}$ & $\theta_{\mathrm{Is}}$ & Israelachvili contact angle \\
\hline$\gamma_{\mathrm{s}}^{\mathrm{p}}$ & $\begin{array}{l}\text { polar component of } \\
\text { solid surface energy }\end{array}$ & $\theta_{\mathrm{r}}$ & receding contact angle \\
\hline$\gamma_{\mathrm{sl}}$ & $\begin{array}{l}\text { solid-liquid interfacial } \\
\text { surface energy }\end{array}$ & $\theta_{\mathrm{Y}}$ & Young's contact angle \\
\hline$\gamma_{\mathrm{sv}}$ & $\begin{array}{l}\text { solid-vapour surface energy } \\
\text { (equivalent to } \gamma_{s} \text { ) }\end{array}$ & $\theta_{1}$ & contact angle of defects \\
\hline$\Phi$ & interaction potential & $\theta_{2}$ & contact angle of basal plane \\
\hline$\lambda$ & $\begin{array}{l}\text { Raman laser wavelength; } \\
\text { inelastic mean free path }\end{array}$ & $\theta_{0}$ & $\begin{array}{l}\text { thermodynamic equilibrium } \\
\text { contact angle }\end{array}$ \\
\hline
\end{tabular}




\subsection{INTRODUCTION}

\subsection{WHAT IS GRAPHITE?}

Many people know graphite as being the black stuff in pencils that allow us to write, typically learning in grade school that "lead pencils" are actually made of graphite and use no actual lead $(\mathrm{Pb})$. Then most people never think of graphite again without realizing how prevalent its use is in our world with applications ranging from a simple writing utensil to being a very good solid lubricant to integration into battery electrodes. ${ }^{1,2}$ As the most stable allotrope of carbon, graphite forms naturally in the Earth and is considered to be the highest grade of coal. Placed under high temperature and extreme pressure $\left(900-1300^{\circ} \mathrm{C}\right.$ and $\left.4.5-6 \mathrm{GPa}\right)$, the crystallographic structure of graphite can rearrange to form diamond. ${ }^{3}$ Graphite and diamond are both carbon allotropes: graphite is $\mathrm{sp}^{2}$-hybridized and diamond is $\mathrm{sp}^{3}$-hybridized.

High quality natural graphite can be found in the Earth and Sri Lanka is the only place in the world where a unique vein-type of natural graphite is found, called Ceylon graphite. The metamorphic terrain of Sri Lanka (under British rule and known as Ceylon until 1972) was particularly ideal for forming the high quality graphite that was required during worldwide industrialization and demand peaked during the World Wars. Ceylon graphite is mined at the Bogala mine in Sri Lanka and was used in many early investigations on the properties of graphitic materials. ${ }^{4}$ 
One of the first synthetic forms of graphite was Kish graphite which is made from molten waste produced during steelmaking. ${ }^{5}$ Over a half century ago, research and production demands led researchers to synthetically fabricate high quality graphitic samples. This lead to a variety of $\mathrm{sp}^{2}$ carbon based materials of which the highest quality is known as highly oriented pyrolytic graphite (HOPG). Pyrolysis $\left(1000-2000^{\circ} \mathrm{C}\right)$ of precursor hydrocarbon gases produce a form of graphite known as pyrolytic carbon or pyrolytic graphite (PG), which is analogous to graphite aside from small amounts of covalent bonding between layers and often times larger interlayer distance due to the absence of high pressure during synthesis. ${ }^{6,7}$ PG is also called turbostratic carbon. Running the pyrolysis procedure under high tensile stress along the c-axis (0.05 $\mathrm{GPa} / 494 \mathrm{~atm}$ ) will force alignment of crystallites forming HOPG through the "stress recrystallization" process described by Blackman and Ubbelohde in $1962 .{ }^{8}$

\subsection{GRAPHITE AND GRAPHENE}

Individual atomically thin layers of graphite are called graphene and graphite can be thought of as being many layers of stacked graphene. In other words, imagine a stack of printer paper. Each piece of paper represents graphene; whereas, the entire stack of paper represents graphite. The amount of pressure applied during synthesis dictates the sample quality yet graphite is always $\mathrm{sp}^{2}$-hybridized carbon. Each graphene layer maintains a six carbon hexagonal lattice, hence it is denoted as h-graphite, and has an $\mathrm{ABAB}$ stacking structure with interlayer spacing of ca. $3.35 \AA$ which changes slightly with temperature. ${ }^{2,9-11}$ Notably, graphite is a lamellar material with weak interlayer bonding due to van der Waals forces and strong intralayer bonding due to non-polar $\mathrm{C}-\mathrm{C}$ bonds. This classifies graphite as part of a larger class of materials known as 
lamellar or van der Waals materials that have strong intralayer bonding and weak interlayer bonding where the uppermost surface can be removed through facile methods such as applying tape and pulling away, thus exposing a fresh or pristine surface. ${ }^{12,13}$

Mosaic spread (mosaicity) of the graphite crystallites is used to quantify the sample quality. ${ }^{14, \ddagger}$ There are three common grades of HOPG commercially available. ZYA is the highest quality and has a mosaic spread of $0.4^{\circ} \pm 0.1^{\circ} .{ }^{17} \mathrm{ZYB}$ and $\mathrm{ZYH}$ are less ordered (i.e., lower quality) HOPG fabricated by applying less pressure during pyrolysis. The lowest quality $\mathrm{sp}^{2}$-hybridzed carbon used in this study is PG which has mosaicity of $30^{\circ}-60^{\circ} .^{2}$ Table 1 shows graphite samples used in this study. Optical and AFM scans of the samples are shown in Figure 1. Salient topographical differences between the samples indicate differences in quality with the highest grade (ZYA) having mostly basal plane surface with few step edges and PG taking a substantially bubbly appearance. The number of defects typically correlates proportionally to the mosaicity of a sample, i.e., a low mosaicity sample will have fewer defects and larger grain sizes than a high mosaicity sample.,

It is worth noting that the optical and AFM images of SPI-1 were taken on a sample that had been cleaved numerous times, thus the surface shows substantially more "damage" than the surface of a brand new sample. Images of the other samples were taken when the sample was relatively new. An important observation made during the writing of this dissertation is that the wettability (fresh and aged surface) is similar on both the brand new surface (new from

\footnotetext{
Interesting science history: Charles Galton (C.G.) Darwin worked alongside Henry Moseley, both English physicists, and wrote two seminal papers on the diffraction characteristics of x-rays in perfect crystals. C.G. Darwin also introduced the concept of mosaicity in crystals. He worked under the great physicists Ernest Rutherford and Niels Bohr at Victoria University of Manchester, which is now the University of Manchester, where Andre Geim and Konstantin Novoselov first isolated graphene nearly a century later. C.G. Darwin is the grandson of the infamous naturalist Charles Darwin and wrote extensively on the concepts of genetics and eugenics (a popular British topic of the time) later in his life. ${ }^{15,16}$
} 
manufacturer) and the surface which has been exfoliated numerous times (see Section 4.2.2 on page 71). During experiments over the past several years, I did not have the foresight to count the number of times each sample was exfoliated and characterize the surface damage along the way. This would have been an interesting contribution when comparing performance of the different samples, especially differences between HOPG samples made by SPI Supplies and Momentive Performance Materials (both brands are purchased through SPI Supplies). The SPI Supplies website states, "It is not a matter that one is better than the other, the quality of both is excellent. However, the two HOPG Brands are made using different processes, and therefore their physical characteristics are a bit different." ${ }^{\text {18 }}$ There is a substantial cost difference between the two manufacturers: ZYA HOPG manufactured by SPI Supplies and Momentive Performance Materials cost $\$ 350(10 \times 10 \times 2 \mathrm{~mm})$ and $\$ 994(12 \times 12 \times 2 \mathrm{~mm})$, respectively, for the same quality sample. Some of this price difference can be due to sample size; however, the substantial price difference leads one to wonder if there are quality differences and, if so, how they affect sample performance.

\subsection{IMPLICATIONS OF SURFACE CONTAMINATION}

Atomically thin materials have inspired many new fields of research within the past decade, ranging from fundamental investigations into material properties to de novo research on ultrathin 2D semiconductors, fabrication of ultrasensitive medical diagnostic devices, and low energy light emitting diodes (LEDs). Graphene has championed research in this field since it was first fabricated; however, its zero bandgap creates many challenges. Transition metal dichalcogenides (TMDCs), e.g., $\mathrm{MoS}_{2}$ and $\mathrm{WS}_{2}$, have a direct bandgap which alleviates the 
challenge of creating a bandgap in graphene-based devices. Graphite, graphene, TMDCs, and rare earth oxides (REOs) are well established as low energy materials that suffer little consequence from hydrocarbon contamination, i.e., the low energy surface has little tendency to adsorb hydrocarbon contaminants. Decades of research on graphite since the $1940 \mathrm{~s}^{19-31}$ has substantiated this belief and only a few researchers ${ }^{32-34}$ - until recently - have provided contradictory evidence. Consequentially, research on 2D and bulk lamellar materials predominately excludes considerations based on hydrocarbon contamination although spontaneous hydrocarbon adsorption is well known to occur on high surface energy metals. ${ }^{35-40}$

Taking into account a thin hydrocarbon layer may help explain prior results on carbon surfaces $^{41}$ and contradictory electrochemical results on the basal plan of HOPG. ${ }^{42}$ McCreery et al. reported that electron transfer on the basal surface of HOPG is 1-3 orders of magnitude slower than electron transfer on glassy carbon. ${ }^{43-47}$ This difference was attributed to variations in the edge plane defect density. ${ }^{48}$ Within the past several years, work by Patel and Unwin et al. has shown that the basal surface of graphite is electrochemically active with electron transfer rates being independent of step edge density. ${ }^{49-55}$ Moreover, they showed that electron transfer rates significantly deteriorate when the graphitic surface is exposed to air; ${ }^{50}$ this has been corroborated through work by Velický and Dryfe et al., ${ }^{56-58}$ Nioradze and Amemiya et al., ${ }^{59-61}$ and $\mathrm{Li}$ and Liu et al. ${ }^{62}$ These results have salient implications for graphene and graphite electrodes and batteries. Taking into account a thin hydrocarbon layer, or controlling to restrict hydrocarbon adsorption, can substantially improve device performance.

Water wettability of a material is an important parameter that describes the degree to which the surface interacts with water. Wettability can then be used to estimate the surface energy of a material along with elucidating how different materials will interact with each other. 
2D materials (i.e., a surface that is one atomic layer thick) have a very high surface-to-volume ratio and any surface changes can drastically affect intrinsic performance of the material, thus wettability of a $2 \mathrm{D}$ material is particularly critical. This behaviour is becoming well established as several research groups have recently shown that contaminant-free surfaces behave fundamentally different than surfaces covered in (aqueous and carbonaceous) contaminants. ${ }^{50,54,}$ 59, 61-65 Recent simulation studies corroborate experimental evidence. ${ }^{22,} 66-69$ Logically, this applies to many other materials such as REOs, TMDCs, and black phosphorous in both their bulk and 2D phase..$^{70-74}$

The following dissertation contains experiments and analysis of several years of work pertaining to the surface properties of graphite and graphene. Specifically, my research group has sought to elucidate the effects of hydrocarbon contamination onto fresh graphitic surfaces and estimate the intrinsic water wettability of $\mathrm{sp}^{2}$-hybridized carbon. To summarize, substantial evidence suggests that hydrocarbons significantly affect the surface properties of graphitic materials and the surface energy of fresh graphite and graphene is consequently reduced due to adsorption of contaminants. ${ }^{75,76}$ This work was inspired by our report (Li et al., Nature Materials 2013, 12, 925-931; doi: 10.1038/nmat3709) which was the first to show that graphene and graphite are mildly hydrophilic and become hydrophobic upon exposure to ambient air and adsorption of airborne hydrocarbons. ${ }^{77}$

Section 2 provides a thorough historical perspective of graphite hydrophobicity (low surface energy) followed by evidence from the past several years indicating that graphitic surfaces may not truly be so hydrophobic. Section 3 expands the investigation to graphene and reviews contradictory experimental and theoretical reports on its hydrophobicity. Then using 
freshly fabricated graphene on copper, contact angle of four liquids was tested and surface free energy was calculated.

The next two sections are new work that has not yet been published in which we seek to determine the influence defects have on wettability. Section 4 investigates the defect density of high and low quality graphite which is then used to help elucidate the intrinsic WCA of fresh $\mathrm{sp}^{2}$-hybridized carbon. Various experimental methods were used to compare graphite samples of varying quality and ultimately Raman spectra of the freshly exfoliated samples were used to calculate their defect density. Section 5 reports our most recent contact angle data and analysis which describes the influence that defects have on wettability. Moreover, the intrinsic water contact angle of fresh (clean) $\mathrm{sp}^{2}$-hybridized carbon is determined.

Lastly, Section 6 reports experimental results on $\mathrm{MoS}_{2}$ which is an important TMDC with properties similar to graphite. The surface energy of fresh and aged graphene, $\mathrm{HOPG}$, and $\mathrm{MoS}_{2}$ was calculated from contact angle data and shows that the fresh surface has higher surface energy and is more hydrophilic than the aged surface. This shows that our findings on graphene and graphite may also be relevant for the myriad of other 2D and bulk materials.

The four appendices detail supplemental experiments which are relevant to the conclusions in the main text of this dissertation. Appendix A discusses details of ellipsometry and Appendix B reports x-ray diffraction data for the graphite samples. Extensive x-ray photoelectron spectroscopy data is shown in Appendix C where the surface chemistry of graphite samples is investigated. The original intention was to use XPS data to determine defect density; however, contamination and lack of surface sensitivity complicated analysis. Additional XPS details for data acquisition and peak fitting are then shown in Appendix D. 
Sections 2,3 , and 6 are taken from previously published material with minor modifications:

- Section 2 and Appendix A is taken from Kozbial et al. Carbon 2014, 74, 218-225

doi: $10.1016 /$ j.carbon.2014.03.025

- Section 3 is taken from Kozbial et al. Langmuir 2014, 30, 8598-8606

doi: $10.1021 / 1 a 5018328$

- Section 6 is taken from Kozbial et al. Langmuir 2015, 31, 8429-8435

doi: 10.1021/acs.langmuir.5b02057

Table 1. Details of graphitic samples. The SPI-1 HOPG sample used for experiments was 10x10x1 mm; whereas, the thicker sample is cited above for a more direct cost comparison. Momentive Performance Materials was previously GE Advanced Ceramics and the samples are analogous.

\begin{tabular}{|c|c|c|c|c|c|}
\hline Sample & Vendor & Size $(\mathbf{m m})$ & Mosaic Spread & Exfoliation & Cost \\
\hline ZYA & Momentive & $12 \times 12 \times 2$ & $0.4^{\circ} \pm 0.1^{\circ}$ & tape & $\$ 994$ \\
\hline ZYH & Momentive & $12 \times 12 \times 2$ & $3.5^{\circ} \pm 1.5^{\circ}$ & tape & $\$ 119$ \\
\hline SPI-1 (ZYA) & SPI & $10 \times 10 \times 1$ & $0.4^{\circ} \pm 0.1^{\circ}$ & tape & $\$ 256$ \\
\hline SPI-2 (ZYB) & SPI & $20 \times 20 \times 2$ & $0.8^{\circ} \pm 0.2^{\circ}$ & tape & $\$ 680$ \\
\hline Pyrolytic (PG) & Graphite Store & $25.4 \times 25.4 \times 3.2$ & $\mathrm{~N} / \mathrm{A}$ & razor & $\$ 109$ \\
\hline
\end{tabular}



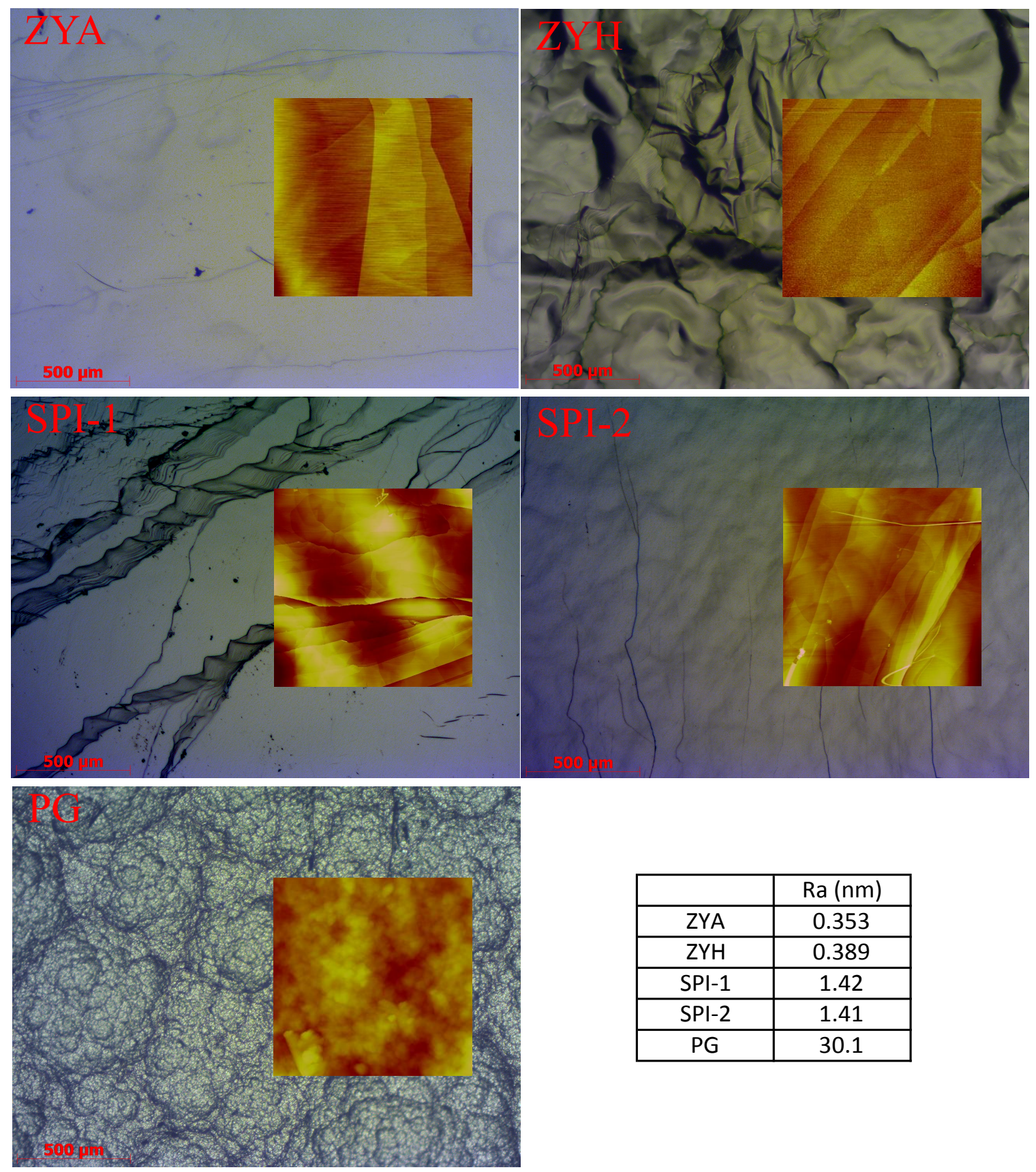

\begin{tabular}{|c|c|}
\hline & Ra $(\mathrm{nm})$ \\
\hline ZYA & 0.353 \\
\hline ZYH & 0.389 \\
\hline SPI-1 & 1.42 \\
\hline SPI-2 & 1.41 \\
\hline PG & 30.1 \\
\hline
\end{tabular}

Figure 1. Optical and AFM images of the graphite samples. Optical images are taken at 5x magnification and the scale bar is $500 \mu \mathrm{m}$. AFM images are $5 \times 5 \mu \mathrm{m}$ with color scale of $10 \mathrm{~nm}$ for HOPG (ZYA, ZYH, SPI-1, and SPI-2) and $500 \mathrm{~nm}$ for PG. 


\subsection{UNDERSTANDING THE INTRINSIC WATER WETTABILITY OF GRAPHITE}

\subsection{HISTORY OF GRAPHITE HYDROPHOBICITY}

Graphite is an important material in the chemical industry with applications in catalyst support and catalysis, ${ }^{78,79}$ gas adsorption, ${ }^{80-82}$ AFM tip calibration, ${ }^{83}$ electrochemistry, ${ }^{48,50,84}$ and battery technology ${ }^{85-88}$ among many others. ${ }^{12,89,90}$ Graphite is also one of the most often used models for studying wetting, interfacial structure of water, and related phenomena. ${ }^{91,92}$ Controlling water wettability is important for product design because changes in water wettability can have significant consequences on adhesion, electrochemical activity, conductivity, and permeation. ${ }^{33}$, 50, 93, 94 Understanding the water wettability of graphite is key for understanding surface properties of other graphitic materials, such as graphene, since it has important implications on applications in tribology ${ }^{95}$ and biodevices. ${ }^{96}$

Since experiments by Fowkes and Harkins in $1940,{ }^{21}$ extensive studies have concluded that graphitic surfaces are hydrophobic with WCA within the $75^{\circ}-95^{\circ}$ range. ${ }^{19-31}$ Early experiments were conducted on natural graphite and later experiments utilized HOPG. Fowkes and Harkins tested Ceylon graphite and reported WCA of $85.3^{\circ}-85.9^{\circ}$ using the tilting plate method. ${ }^{21}$ Morcos reported WCA of $84.2^{\circ}$ on exfoliated graphite determined by the indirect

\footnotetext{
$\S$ Published previously: Kozbial, A.; Li, Z.; Sun, J.; Gong, X.; Zhou, F.; Wang, Y.; Xu, H.; Liu, H.; Li, L. Understanding the Intrinsic Water Wettability of Graphite. Carbon 2014, 74, 218-225. ${ }^{76}$
} 
meniscus height method where the sample was partially immersed in water. ${ }^{24}$ Morcos also reported WCA of $83.9^{\circ}$ on highly oriented graphite by advancing meniscus method. ${ }^{25}$ More recently, WCAs determined by static sessile drop were reported as $91^{\circ},{ }^{28} 79^{\circ},{ }^{30}$ and $75^{\circ 31}$ on exfoliated HOPG, $98.3^{\circ}$ on graphite, ${ }^{23}$ and $91.0^{\circ}$ on HOPG aged in air for days. ${ }^{26}$ Adamson and Gast reported advancing WCA of $86^{\circ}$ on graphite. ${ }^{29}$ Raj et al. recently reported advancing WCA on HOPG to be $c a \cdot 91^{\circ} \cdot{ }^{27}$ Hydrophobicity has also been observed on other graphitic materials. Aligned carbon nanotubes have shown superhydrophobic behavior where WCAs of $163.4^{\circ 97}$ and $158.5^{\circ 98}$ were observed. Additionally, as-grown carbon nanotube forests exhibit WCA of $161^{\circ} .^{20}$ Raj et al. reported advancing WCA of monolayer graphene on $\mathrm{SiO}_{2}$ substrate of $c a .90^{\circ}$, similar to that of graphite. ${ }^{27}$

Despite the dominant view that graphite is hydrophobic, a few studies have reported evidence of a much more hydrophilic surface with WCA of $35^{\circ}-65^{\circ} .{ }^{32-34,}, 77,99,100$ In 1975 , Schrader reported that WCA of exfoliated graphite was $35^{\circ} \pm 4^{\circ}$ under an ultrahigh vacuum (UHV) environment and ion bombardment of the surface decreased WCA to $0^{\circ} .{ }^{33}$ Subsequent experiments indicated that ion bombardment damaged the graphite surface; however, the $35^{\circ}$ WCA was determined on a surface exposed only to bakeout temperature up to $750^{\circ} \mathrm{C}$ under UHV and the surface was verified by low energy electron diffraction to be clean and not damaged. ${ }^{33,}{ }^{34}$ Schrader proposed that the "hydrophobicity" reported is due to the airborne hydrocarbon contamination. ${ }^{33}$ Additionally, Tadros et al. used the captive bubble method and reported advancing WCA of $63^{\circ}-65^{\circ}$ on pyrolytic high-density isotropic carbon. ${ }^{32}$ Luna et al. also found WCA of $\mathrm{ca} .30^{\circ}$ on graphite utilizing scanning force microscopy techniques. ${ }^{100}$ Cao et al. discovered that water on HOPG adsorbs as nanodrops with WCA of less than $10^{\circ}$ under 
microscopic conditions. ${ }^{99}$ Very recently, three reports also suggested contamination of graphitic surfaces by airborne hydrocarbons. ${ }^{66,77,101} \mathrm{Li}$ et al. reported WCA of HOPG to be $64.4^{\circ}$ immediately after exfoliation, ${ }^{77}$ which again suggests that graphite is intrinsically more hydrophilic than previously believed. Using Kelvin probe microscopy and mass spectroscopy, Gomez-Herrero et al. showed that airborne hydrocarbons adsorb onto graphitic surfaces. ${ }^{101}$ Furthermore, $\mathrm{Wu}$ and Aluru used MD simulations to model hydrocarbon contamination by ethane molecules and showed that graphitic surfaces are intrinsically hydrophilic and become hydrophobic after hydrocarbon contamination ${ }^{66}$.

The concept that graphite is (mildly) hydrophilic has not been accepted by the scientific community; one of the reasons is likely due to the issues of earlier experimental procedures. ${ }^{33}$ For example, a report has directly challenged the validity of Schrader's results ${ }^{92}$ since Schrader determined WCA in an UHV environment, ${ }^{33}$ which is known to result in lower WCA due to rapid water evaporation. ${ }^{102,103}$ To address this issue, here we present a study focusing on temporal monitoring of exfoliated HOPG in ambient air by WCA and attenuated total reflectance-Fourier transform infrared spectroscopy (ATR-FTIR), following the experimental procedure established in our previous work. ${ }^{77}$

\subsection{TIME EVOLUTION OF WCA AND HYDROCARBON ADSORPTION}

Static WCA measured on a freshly exfoliated (SPI-2) HOPG is shown in Figure 2a. Before exfoliation the WCA on HOPG exposed to air was $92.8^{\circ} \pm 2.7^{\circ}$. Within 10 seconds after exfoliation the static WCA was measured to be $64.4^{\circ} \pm 2.9^{\circ}$ and increased within the next $\sim 15$ min to $c a .90^{\circ}$ which remained constant for $60 \mathrm{~min}$. After 7 days of exposure to air, the static 
WCA recovered to $97.0^{\circ} \pm 1.8^{\circ}$. This trend was consistently observed although the rate of WCA increase would change with lab location, HOPG sample, and time of year.

ATR-FTIR was used to characterize the HOPG surface in order to identify the cause of WCA change. The spectra indicate that hydrocarbon adsorption occurs when fresh HOPG is exposed to air. Shown in Figure $2 \mathrm{~b}$, peaks at $2850 \mathrm{~cm}^{-1}$ and $2930 \mathrm{~cm}^{-1}$ are observed on aged HOPG and are absent on freshly exfoliated HOPG. These peaks correspond to symmetric and asymmetric stretching of the methylene group, respectively. ${ }^{104,}{ }^{105}$ Presence of the methylene stretching peaks indicate hydrocarbon contamination of the surface. Since the initially fresh (clean) sample was exposed only to air during aging, the source of hydrocarbon contamination is expected to be airborne. ${ }^{33,77,106}$
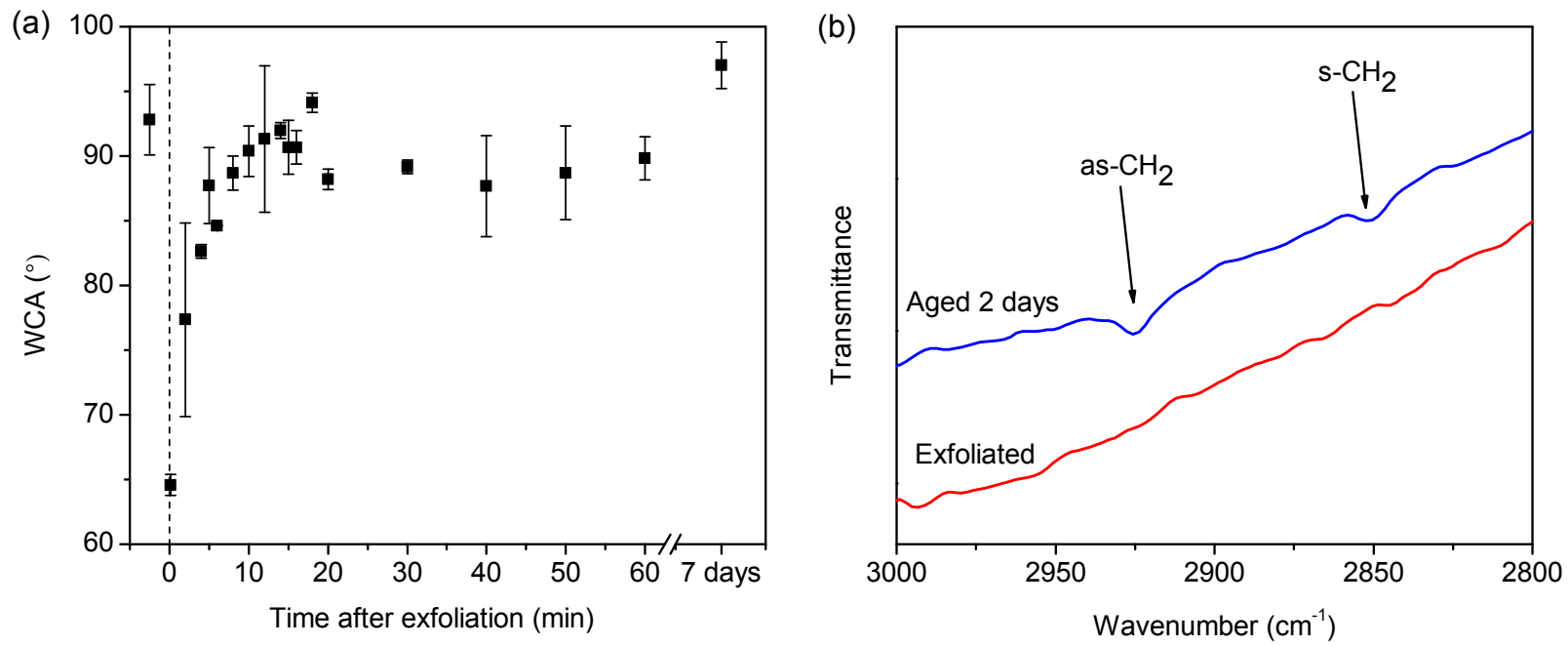

Figure 2. WCA and ATR-FTIR of fresh and aged HOPG. (a) Static WCA of exfoliated HOPG in air where the dotted line indicates exfoliation. (b) ATR-FTIR of aged and freshly exfoliated HOPG. s- $\mathrm{CH}_{2}$ at $2850 \mathrm{~cm}^{-1}$ indicates symmetric methylene stretching and as- $\mathrm{CH}_{2}$ at $2930 \mathrm{~cm}^{-1}$ indicates asymmetric methylene stretching. 
Ellipsometry was used to study the kinetics of hydrocarbon adsorption (details presented in Appendix A). Temporal in situ monitoring of exfoliated HOPG is shown in Figure 3a. In ambient air, linear growth of an adsorbed hydrocarbon layer occurs within the first 30 minutes reaching a thickness $c a .0 .45 \mathrm{~nm}$ and continues to slowly increase approaching $0.50 \mathrm{~nm}$ after 120 minutes. A previous report of organic deposition on $\mathrm{SiO}_{2}$ monitored by ellipsometry shows initial linear thickness increase followed by a significant decrease in adsorption kinetics, similar to our results on HOPG. ${ }^{105}$ Similar to WCA increase, this trend was consistently observed although the rate of increase could change with lab location, HOPG sample and time. When the WCA and ellipsometry tests were conducted on the same HOPG sample, in the same lab (room) and in the same day, the kinetics is quite similar, as shown in Figure 3b, indicating the WCA increase results from the airborne hydrocarbon contamination.

To show that hydrocarbons do adsorb onto the graphite surface, freshly exfoliated HOPG was intentionally exposed to 1-octadecene vapor. Within 20 minutes, a hydrocarbon layer of $c a$. $0.50 \mathrm{~nm}$ adsorbed onto the HOPG surface (Figure 3a). The film thickness continues to increase to $c a .0 .90 \mathrm{~nm}$ and remains constant after 80 minutes, indicating possible saturation of the exposed HOPG surface at the current conditions. WCA of exfoliated HOPG exposed to a saturated 1-octadecene vapor exhibits kinetics similar to HOPG exposed to ambient air within the first 15 minutes and continues to increase approaching $104^{\circ}$ after 24 hours. In comparison, HOPG aged in air for 7 days showed a WCA of $97.0^{\circ} \pm 1.8^{\circ}$. These experiments provide further evidence that freshly exfoliated HOPG becomes contaminated by airborne hydrocarbons and the hydrophobic properties of HOPG are a consequence of adsorbed hydrocarbons. 

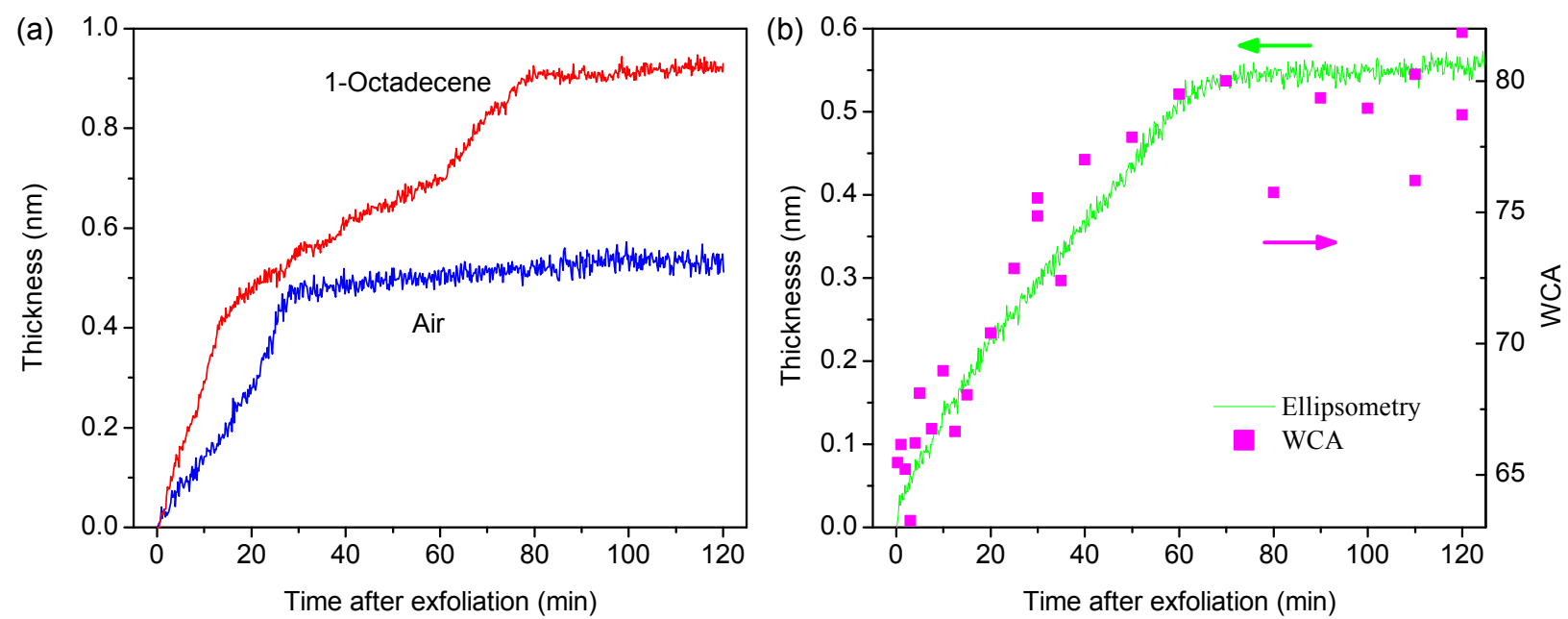

Figure 3. In situ ellipsometry of fresh HOPG. (a) Ellipsometry of adsorbed hydrocarbon layer thickness on exfoliated HOPG after exposure to ambient air (blue) and 1-octadecene reservoir open to ambient air (red). (b) WCA and ellipsometry measurements of HOPG taken on the same sample, on the same day, and in the same lab. The HOPG was exfoliated at time 0 before the

first measurement.

\subsection{REMOVAL OF HYDROCARBON CONTAMINANTS}

Adsorbed hydrocarbons can be removed by ultra-high vacuum (UHV) treatment. To show this was the case, HOPG was exfoliated and exposed to ambient air for 2 weeks. The sample was then placed into a UHV chamber (less than $1 \times 10^{-9}$ torr) for 15 hours, after which the WCA decreased to $59^{\circ}$ and recovered to $c a .85^{\circ}$ after 10 minutes of exposure to ambient air (Figure 4a). The adsorbed hydrocarbons can also be removed by a brief $\mathrm{UV} / \mathrm{O}_{3}$ treatment to recover the hydrophilicity of HOPG. In one experiment, HOPG was exfoliated and placed in a saturated 1octadecene environment for 1 hour before it was treated with $\mathrm{UV} / \mathrm{O}_{3}$ for 5 minutes (Figure $4 \mathrm{~b}$ ). The WCA after 1 -octadecene exposure was $101.2^{\circ} \pm 0.5^{\circ}$. WCA measured about 10 seconds after $\mathrm{UV} / \mathrm{O}_{3}$ was $32^{\circ}$ and increased to $\mathrm{ca}$. $80^{\circ}$ after 60 minutes. These results indicate that 
$\mathrm{UV} / \mathrm{O}_{3}$ removes the original 1-octadecene hydrocarbon on $\mathrm{HOPG}$ and adsorption is reproducible when the $\mathrm{UV} / \mathrm{O}_{3}$ cleaned $\mathrm{HOPG}$ is exposed to airborne hydrocarbons.

We note that defects could form on the HOPG surface after $\mathrm{UV} / \mathrm{O}_{3}$ exposure and may account for the much lower WCA observed here and also enhance hydrocarbon adsorption. ${ }^{107}$ To evaluate this possibility, micro-Raman spectra were taken to determine the effect of exfoliation and $\mathrm{UV} / \mathrm{O}_{3}$ treatment on the surface quality of our HOPG. Spectra shown in Figure 5 indicate that high surface quality HOPG was maintained throughout our experiments. The G and 2D peak intensity remain similar after $\mathrm{UV} / \mathrm{O}_{3}$ treatment and the $\mathrm{D}$ peak at $1350 \mathrm{~cm}^{-1}$ is inconspicuous. Intensity ratio of the D peak to the G peak is 0.010 . It is important to note that Raman spectroscopy on HOPG is not surface sensitive: while the D peak presumably comes from only the top layer of graphene, the G peak will have contribution from many more sublayers. After correcting for this effect, the estimated surface defect density is less than $(5.1 \pm$ 1.4) $\times 10^{10} \mathrm{~cm}^{-2}$, or less than $(1.3 \pm 0.4) \times 10^{-5}$ defect per carbon atom. These results indicate that surface disorder was negligible ${ }^{108}$ and that hydrocarbon adsorption can be reversed by $\mathrm{UV} / \mathrm{O}_{3}$ treatment. 

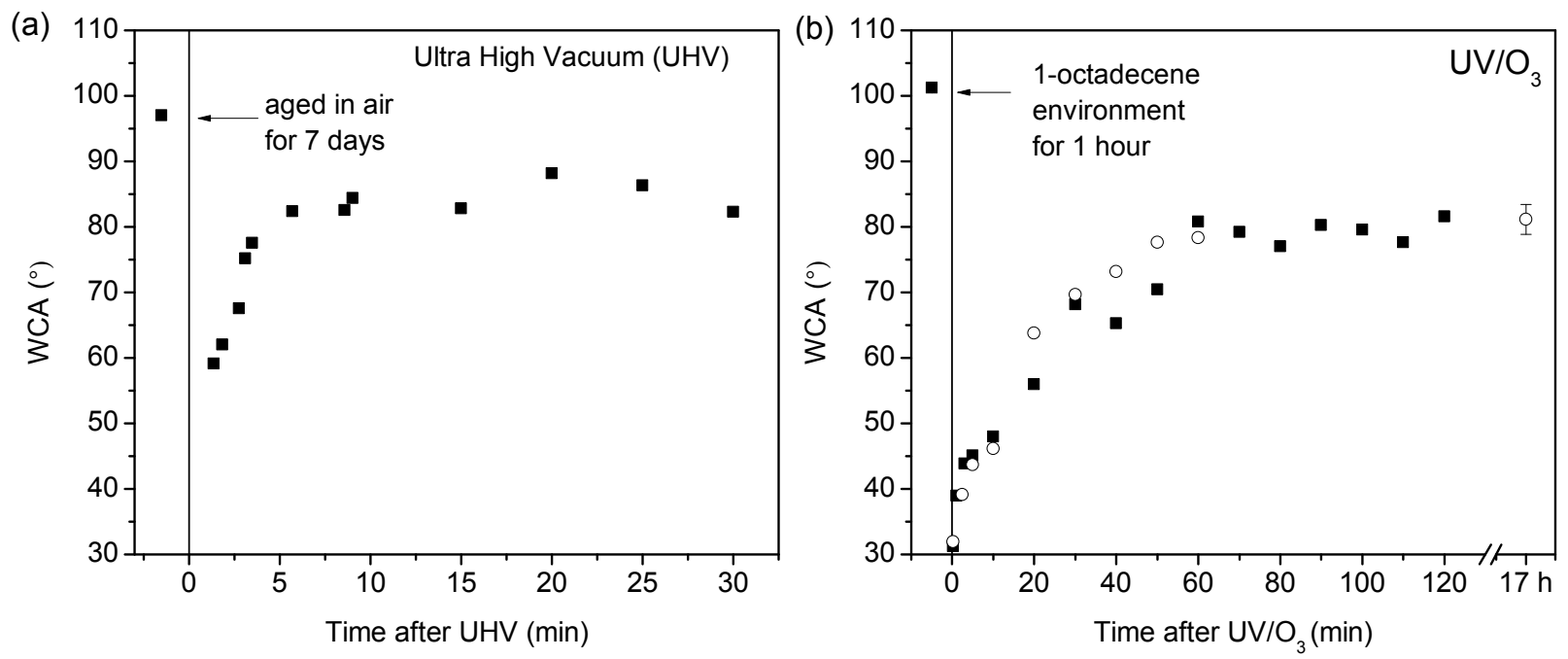

Figure 4. Removal of hydrocarbon contaminants. (a) Static WCA of exfoliated HOPG exposed to ambient air for 2 weeks then ultra high vacuum (UHV) for 15 hours. (b) Static WCA of exfoliated $\mathrm{HOPG}$ exposed to $\mathrm{UV} / \mathrm{O}_{3}$ for $5 \mathrm{~min}$. Solid squares: exfoliated $\mathrm{HOPG}$ exposed to saturated 1-octadecene vapor for 1 hour then $5 \mathrm{~min} \mathrm{UV} / \mathrm{O}_{3}$ treatment. Open circles: exfoliated HOPG exposed to $5 \mathrm{~min} \mathrm{UV} / \mathrm{O}_{3}$ treatment.

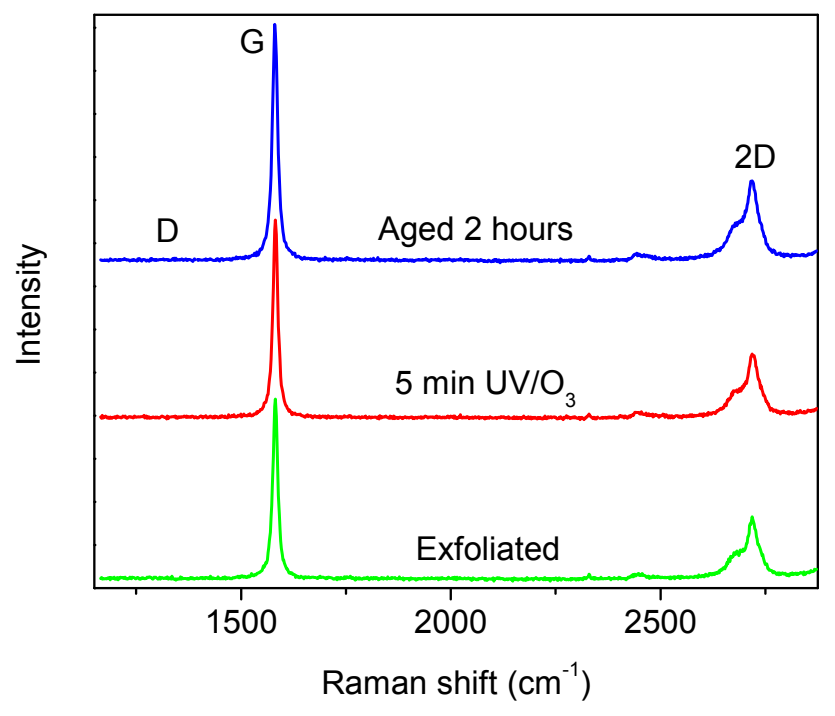

Figure 5. Micro-Raman spectra of HOPG aged in ambient air for 2 hours, after 5 min $\mathrm{UV} / \mathrm{O}_{3}$ exposure, and after exfoliation. Data collected with a 30 second acquisition time. 


\subsection{INTRINSIC WCA OF GRAPHITE}

Our results show that the surface of freshly exfoliated HOPG is intrinsically more hydrophilic than previously believed and hydrocarbon adsorption results in surface contamination and hydrophobicity of HOPG. We found that the WCA of HOPG was $64.4^{\circ} \pm 2.9^{\circ}$ within 10 seconds of exfoliation and increased to $c a .90^{\circ}$ with aging time. Although previous work has suggested that hydrocarbon contamination could affect wettability of $\mathrm{HOPG},{ }^{33,34,77}$ our work is the first to provide direct evidence for the presence of such contamination and to correlate between wetting behavior and surface contamination of graphite. Our reported initial WCA on exfoliated HOPG of $64.4^{\circ}$ is greater than the $35^{\circ}$ value reported by Schrader, ${ }^{33}$ possibly caused by differences in experimental procedure. Schrader measured WCA by introducing water vapor into an UHV chamber and using a cold finger to condense water and deliver it to the testing surface. ${ }^{33}$ It is known that WCA should be lower in UHV due to water evaporation. ${ }^{102,} 103$

In contrast, our HOPG was exfoliated in ambient air and the first test was taken within 10 seconds of exfoliation. Our ellipsometry data shows that the surface becomes saturated with a hydrocarbon film after $c a$. 30-90 min of air exposure. Assuming adsorption kinetics is linear immediately after exfoliation, we estimate the surface coverage of hydrocarbon was less than $0.6 \%$ of the saturation coverage at 10 seconds after exfoliation, when the first WCA was taken. Therefore, our WCA data could reflect the intrinsic wettability of HOPG if the following two conditions can be excluded: (1) Within the first 10 seconds, the adsorption kinetics is nonlinear. (2) The $0.6 \%$ of the saturation coverage of adsorbed hydrocarbons could strongly affect WCA.

Our results show that both $\mathrm{UV} / \mathrm{O}_{3}$ and $\mathrm{UHV}$ remove hydrocarbon contaminants resulting in a hydrophilic HOPG surface. UHV causes desorption of hydrocarbons from the HOPG 
surface; whereas, $\mathrm{UV} / \mathrm{O}_{3}$ treatment cleaves the $\mathrm{C}-\mathrm{C}$ and $\mathrm{C}-\mathrm{H}$ bonds of hydrocarbon into volatile

components which are removed without further processing. ${ }^{33,107}$ Both methods provide a more hydrophilic HOPG surface by removing hydrocarbons, yet $\mathrm{UV} / \mathrm{O}_{3}$ treatment resulted in a WCA of $c a .30^{\circ}$ (Figure $4 \mathrm{~b}$ ) while the WCA after UHV treatment was $59^{\circ}$ (Figure $4 \mathrm{a}$ ). This difference could be explained by the hydrophilic moiety on the HOPG surface, which results from the chemical reaction between adsorbed hydrocarbons induced by $\mathrm{UV} / \mathrm{O}_{3}$ treatment.

\subsubsection{Theoretical calculation}

Theoretical calculations were carried out to estimate the effect of a sub-nanometer thick hydrocarbon film on the wettability of HOPG. Our calculations assume that interactions between water and all substrates are van der Waals in nature and additive. We modeled graphite with a slab of 100 layers of graphene. Based on the WCA of clean graphite, $64.4^{\circ}$, and using the modified Young-Dupré equation, ${ }^{109,} 110$ we can calculate the interaction potential per unit area between a water drop and graphite following a previously reported procedure. ${ }^{111}$ From this potential, we can extract the van der Waals interaction parameter between one water molecule and one graphene layer. The calculation details can be found elsewhere. ${ }^{77}$ We carried out similar calculations to extract the van der Waals interaction parameter between one water molecule and one $-\mathrm{CH}_{2}$ - using the experimental WCA on polyethylene $\left(96^{\circ}-105^{\circ}\right),{ }^{112-115}$ and crystal structure of polyethylene. $^{116,117}$

The interaction potential per unit between one water molecule and one flat polyethylene film, $w_{C L}$, is given by: 


$$
w_{C L}(z)=\frac{-\pi \sigma A}{2 z^{4}} \quad \text { Equation } 1
$$

where (i) $\sigma$ is the surface density of $-\mathrm{CH}_{2}$ - units of single-crystal polyethylene with $\sigma=2 /(a *$ $b$ ), where $a=4.93 \AA$ and $b=2.55 \AA$ are the lattice constants of polyethylene, ${ }^{117}$ (ii) $z$ is the shortest distance between water and polyethylene surface, and (iii) $A$ is the van der Waals interaction parameter between one water molecule and one $-\mathrm{CH}_{2}$ - group.

For a water drop placed on an infinitely large polyethylene film, the total interaction potential per unit area between bulk water and N-layer polyethylene film, $\Phi_{N C L}$, is given by:

$$
\Phi_{N C L}=\sum_{n=1}^{N} \int_{\delta+(n-1) d}^{\infty} \rho(z) w_{C L}(z) d z \quad \text { Equation } 2
$$

where $\rho(z)$ is the water molecule number density and satisfies Boltzmann distribution, $\delta$ is the shortest distance between water and polyethylene film $(3.25 \AA),{ }^{118}$ and $d$ is the interlayer distance between stacked polyethylene layers $(3.7 \AA) .{ }^{117}$ Using the literature reported WCA of polyethylene film $\left(96^{\circ}-105^{\circ}\right),{ }^{112-115}$ we calculated $\Phi_{N C L}$ from the modified Young Dupré equation:

$$
\gamma(1+\cos \theta)=-\Phi_{N C L} \quad \text { Equation } 3
$$

where $\gamma$ is the surface tension of water $\left(72.8 \mathrm{~mJ} / \mathrm{m}^{2}\right)$, and $\theta$ is the WCA. By fitting the calculated $\Phi_{N C L}$ to Equation 2, the related van der Waals interaction parameter (A) can be determined to be $18.96,18.39,17.80,17.18 \mathrm{eV} / \AA^{6}$, corresponding to WCA of $96^{\circ},{ }^{112} 99^{\circ},{ }^{113} 102^{\circ},{ }^{115} 105^{\circ},{ }^{114}$ respectively. 
With these two van der Waals interaction parameters, we calculated the interaction potential per unit area between a water drop and graphite contaminated by a thin layer of $-\mathrm{CH}_{2}-$. We introduced N-layer stacked polyethylene chains between the HOPG surface and water drop. To simplify the calculation, we define the layers of HOPG to be 100 and consider the total interaction potential between bulk water and the entire polyethylene covered HOPG surface, $\Phi_{\text {total }}$, to be given by:

$$
\begin{gathered}
\Phi_{\text {total }}=\Phi_{H O P G L}+\Phi_{N C L}= \\
\sum_{n=1}^{100} \int_{\delta H L+(n-1) * d H}^{\infty} \rho(z) w_{H L}(z) d z+\sum_{n=1}^{N} \int_{\delta C L+(n-1) * d N C}^{\infty} \rho(z) w_{N C L}(z) d z \quad \text { Equation } 4
\end{gathered}
$$

where (i) $\rho(z)=\rho_{\mathrm{L} 0} \exp \left[-w_{\text {total }}(z) / \mathrm{k}_{\mathrm{B}} \mathrm{T}\right]$, (ii) $w_{\text {total }}(z)=w_{H L}(z)+w_{N C L}(z)$, (iii) $\delta_{\mathrm{HL}}=\delta_{\mathrm{CL}}+$ $(\mathrm{N}-1) * \mathrm{~d}_{\mathrm{NC}}+\delta_{\mathrm{HC}}$, (iv) $\delta_{\mathrm{HC}}(3.7 \AA)$ is the equilibrium contact separation between top layer of HOPG and bottom layer of polyethylene film, ${ }^{119}$ and (v) $\delta_{\mathrm{CL}}(3.25 \AA),{ }^{118} \mathrm{~d}_{\mathrm{H}}(3.35 \AA)$, and $\mathrm{d}_{\mathrm{NC}}$ $(3.7 \AA)^{117}$ is the shortest distance between water and polyethylene film, the interlayer distance between HOPG layers, and crystalline polyethylene layers, respectively. Combining with the modified Young-Dupré equation, the water contact angle $(\theta)$ on polyethylene covered HOPG surface was calculated as a function of number of polyethylene layers. Finally, we converted the calculated potential to WCA using the modified Young-Dupré equation.

The calculation results are shown in Figure 6. Theoretical model of WCA on polyethylene (PE) where the WCA on HOPG increases from $64.4^{\circ}$ to $96-105^{\circ}$ as the graphite surface becomes covered with an increasingly thick film of $-\mathrm{CH}_{2-}$. Interestingly, only 2 monolayers $(\sim 7 \AA)$ of $-\mathrm{CH}_{2}$ - are enough to induce this substantial WCA increase. Our experimental data shows a WCA of $c a .90^{\circ}$ for a HOPG surface covered with a $0.45 \mathrm{~nm}$ thick of 
airborne hydrocarbon contamination layer; in the case of 1-octadecene contaminated HOPG, a $0.9 \mathrm{~nm}$ thick hydrocarbon layer results in WCA of $101^{\circ}$. Both results are in excellent agreement with our theoretical estimation (see Figure 2a and Figure 3a).
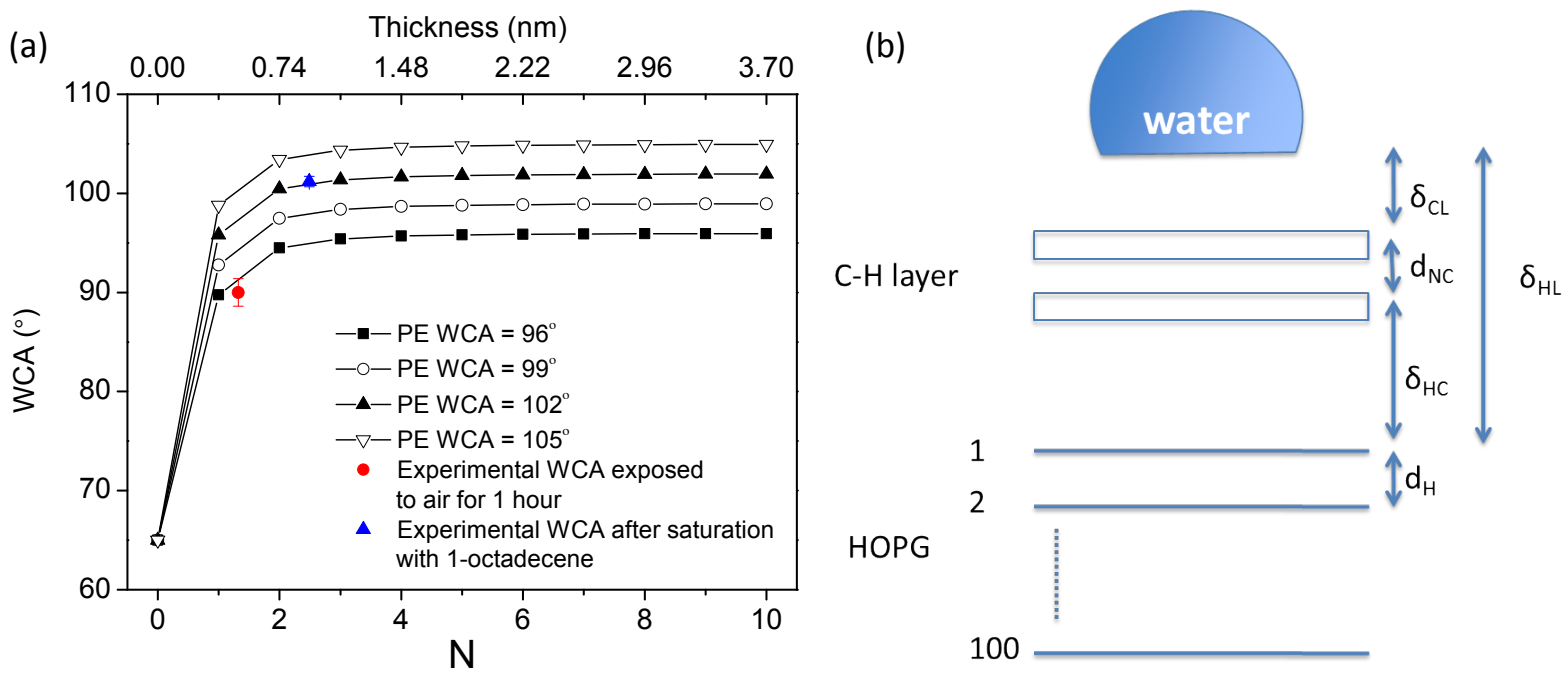

Figure 6. Theoretical model of WCA on polyethylene (PE). (a) Calculated WCA as a function of $\mathrm{N}$ layers of $-\mathrm{CH}_{2}-$ on HOPG surface at different polyethylene (PE) WCAs. The red circle is the experimental WCA of HOPG after 1 hour exposure to ambient air. The blue triangle is the experimental WCA of HOPG at saturated conditions after 1-octadecene exposure. (b) Schematic of model used to determine the interactions between a water drop and HOPG. "C-H layer" refers to $-\mathrm{CH}_{2}$ - hydrocarbon contamination.

\subsection{CONCLUSIONS}

In conclusion, we presented a detailed study on the wetting properties of HOPG exfoliated in air.

Our results show that adsorbed airborne hydrocarbon has a profound impact on the wettability of graphite and indicate that a clean HOPG surface is intrinsically hydrophilic with a WCA of $64.4^{\circ}$ $\pm 2.9^{\circ}$. We provide direct experimental evidence and theoretical justification that the previously 
reported hydrophobic behavior of HOPG is due to hydrocarbon contamination. Our finding is in line with recent reports ${ }^{49,50,52,62}$ showing that electron transfer kinetics on the HOPG surface changes dramatically after exposure to the ambient environment, which is possibly due to the adsorption of the airborne contaminants. Given their widespread use in research and development, our results call for a reevaluation of the wetting and other fundamental surface properties of graphitic materials. 


\subsection{DETERMINING THE SURFACE ENERGY OF GRAPHENE ${ }^{* *}$}

\subsection{HISTORY OF GRAPHENE HYDROPHOBICITY}

Graphene exhibits interesting electrical, optical, mechanical, and chemical properties which make it a candidate material for many important applications, including transistors, sensors, transparent conductors, and clean energy devices. ${ }^{95,120-124}$ Due to its atomic thinness, graphene must be bound to at least one substrate for most applications. As a result, the adhesion between graphene and other materials is critical for device fabrication. ${ }^{23,28,125,126}$ Since adhesion is largely dependent on surface energy, it is important to determine the surface energy of graphene. Moreover, surface energy is also critical to the adsorption process, which has been shown to affect the properties of graphene. ${ }^{127}$

Although several research groups have conducted contact angle measurements on graphene, analysis was seldom extended to surface energy calculations. Shin et al. studied epitaxial graphene grown on $\mathrm{SiC}$ and reported WCA on the graphene as $92^{\circ} \cdot{ }^{28} \mathrm{Kim}$ et al. reported WCA of CVD-grown graphene transferred to $\mathrm{SiO}_{2}$ as $90.4^{\circ}$ and $93.8^{\circ}$ for Ni-grown and $\mathrm{Cu}$-grown graphene, respectively. ${ }^{128}$ Moreover, Rafiee et al. reported WCA of G/Cu to be $86^{\circ} .^{123}$ These results on single- and multi-layer graphene are generally consistent with

*** Reprinted with permission from Kozbial, A.; Li, Z.; Conaway, C.; McGinley, R.; Dhingra, S.; Vahdat, V.; Zhou, F.; D’Urso, B.; Liu, H.; Li, L. Study on the Surface Energy of Graphene by Contact Angle Measurements. Langmuir 2014, 30, 8598-8606. ${ }^{75}$ Copyright 2014 American Chemical Society. 
hydrophobic WCA values of graphite..$^{21,23,28,29,77}$ In 2013, Li et al. showed that WCA of CVDgrown $\mathrm{G} / \mathrm{Cu}$ tested within 10 seconds after synthesis was $44^{\circ}$ and increased to $80^{\circ}$ after 1 day exposure in ambient air. The increase of hydrophobicity was attributed to the adsorption of airborne hydrocarbon contaminants onto the initially clean surface and this conclusion was supported by ATR-FTIR and x-ray photoelectron spectroscopy (XPS) experiments. ${ }^{77}$ A parallel study by Gomez-Herrero et al. also concluded that graphite is contaminated by hydrocarbons using Kelvin probe force microscopy and mass spectrometry techniques. ${ }^{101}$ Accordino et al. used molecular dynamics simulations to show that the water density profile for graphene is consistent with a hydrophilic system. ${ }^{67}$

Although WCA data provides valuable information on the wettability of a surface, many other surface properties are related to the surface free energy. Surface energy $(\gamma)$ is a key parameter characterizing a solid surface and its interaction with other materials. It has been well recognized that this parameter is closely related to not only wettability but also other important properties at the surface interface, e.g., adhesion and friction. ${ }^{129,130}$ Therefore, measuring the surface energy of graphene is important for both fundamental study and applications. ${ }^{131}$ Although numerous studies on the electrical and optical properties of graphene have been reported in literature, to date only a few published reports have characterized the surface free energy of graphene. Wang et al. produced graphene sheets through chemical exfoliation of a natural graphite flake and hydrazine conversion and then assembled graphene sheets into a film. They determined the surface energy of the graphene film by contact angle measurements using the Neumann model and concluded that surface energy of the graphene film and graphite was $46.7 \mathrm{~mJ} / \mathrm{m}^{2}$ and $54.8 \mathrm{~mJ} / \mathrm{m}^{2}$, respectively. ${ }^{23}$ Based on the exfoliation experiments of graphite in 
various solvents, Coleman et al. reported surface energy values of $65-90 \mathrm{~mJ} / \mathrm{m}^{2}$ for graphene and graphite. $^{132,133}$

Contact angle testing, schematically shown in Figure 7, is experimentally simpler than most other characterization methods. ${ }^{134,}{ }^{135}$ Usually, several testing liquids with different polar and dispersive components are used for testing. ${ }^{136}$ Table 2 lists the polar and dispersive surface energy components of the four test liquids used in this study: water (W), diiodomethane (DIM), ethylene glycol (EG), and glycerol $(\mathrm{G}) .{ }^{125}$ Several models have been proposed to translate contact angle data to surface energy and the validity of these models is still a matter of debate. ${ }^{125,}$ 137 In this study, the surface free energy has been evaluated from contact angle data using the Neumann, ${ }^{138}$ Fowkes, ${ }^{139}$ and Owens-Wendt (extended Fowkes) ${ }^{129}$ models.

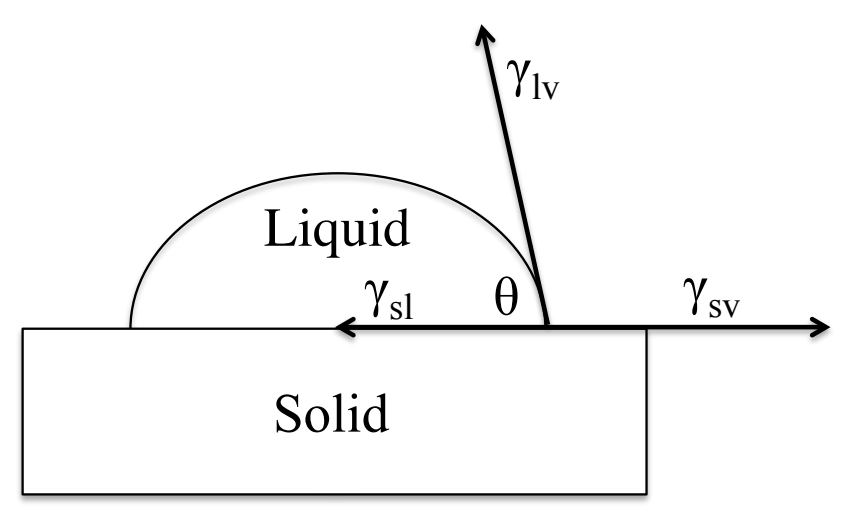

Figure 7. Schematic depicting the interfaces of a liquid drop on a solid surface. ${ }^{140}$ 
Table 2. Polar and dispersive surface energy components of test liquids.

\begin{tabular}{|c|c|c|c|}
\hline & $\begin{array}{c}\gamma_{1} \\
\left(\mathrm{~mJ} / \mathrm{m}^{2}\right)\end{array}$ & $\begin{array}{c}\gamma_{1}^{\mathrm{p}} \\
\left(\mathrm{mJ} / \mathrm{m}^{2}\right)\end{array}$ & $\begin{array}{c}\gamma_{1}^{\mathrm{d}} \\
\left(\mathrm{mJ} / \mathrm{m}^{2}\right)\end{array}$ \\
\hline Water & 72.8 & 51.0 & 21.8 \\
\hline Diiodomethane & 50.8 & 0.0 & 50.8 \\
\hline Ethylene glycol & 48.0 & 19.0 & 29.0 \\
\hline Glycerol & 64.0 & 30.0 & 34.0 \\
\hline
\end{tabular}

\subsection{SURFACE FREE ENERGY MODELS}

Calculating the surface free energy from contact angle measurements is a powerful technique for elucidating interfacial surface properties. Thomas Young published An Essay on the Cohesion of Fluids in 1805 which related wettability to solid surface tension. ${ }^{141}$ Young's equation states that solid surface energy $\left(\gamma_{\mathrm{s}}\right.$ or $\left.\gamma_{\mathrm{sv}}\right)$ is related to the solid-liquid surface energy $\left(\gamma_{\mathrm{sl}}\right)$, liquid surface energy $\left(\gamma_{1}\right.$ or $\left.\gamma_{1 v}\right)$, and contact angle $(\theta)$ :

$$
\gamma_{s}=\gamma_{s l}+\gamma_{l} \cos \theta \quad \text { Equation } 5
$$

Liquid surface tension $\left(\gamma_{1}\right)$ is easily evaluated using a tensiometer and reliable data can be found in literature for nearly every test liquid. The contact angle $(\theta)$ is evaluated as shown in Figure 7. This leaves two unknown quantities: $\gamma_{s}$ and $\gamma_{s l}$. There is no experimental method to directly evaluate $\gamma_{\mathrm{s}}$ and $\gamma_{\mathrm{sl}}$, thus several models have been developed which solve for $\gamma_{\mathrm{sl}}$ then 
allow $\gamma_{\mathrm{s}}$ to be calculated by Equation 5. Further details can be found in several literature reviews. $^{137,142-145}$

Kwok and Neumann presented general guidelines for calculating surface energy from contact angle data: ${ }^{146}$

1. Assume Young's equation is valid.

2. Only pure liquids are to be used, no mixtures or surfactants.

3. No chemical or physical reactions occurring. $\gamma_{\mathrm{sv}}, \gamma_{\mathrm{sl}}$, and $\gamma_{\mathrm{lv}}$ are assumed to be constant during measurement.

4. Surface tension of test liquid must be greater than surface energy of solid, else liquid will completely spread.

5. $\gamma_{s}$ is assumed to remain constant and independent of test liquid.

The solid surface must be inert to the test liquids and not absorptive. A variety of test liquids with a wide range of surface tension values should be used to obtain robust results; calculation results can be influenced by the choice of test liquids. ${ }^{137}$ This issue can be mitigated by directly comparing only data collected using (a) the same test liquids and (b) calculated using the same model, thus illustrating the importance of considering multiple models and a breadth of liquids when calculating surface energy. The objective of this study was not to determine validity of the models, which has been a matter of debate for decades. Instead, the intention is to utilize commonly accepted models, estimate surface energy of graphitic samples, and understand key factors controlling surface energy.

In the current investigation, three models - Neumann, ${ }^{138}$ Fowkes, ${ }^{139}$ and Owens-Wendt (extended Fowkes), ${ }^{129}$ - were used to calculate surface energy of graphene from contact angle data. The Fowkes and Owen-Wendt models allow for dissociation of the total surface energy into polar and non-polar (dispersive) components which provides further insight into wetting 
behaviour. $^{129,139}$ There is no scientific preference of one model over another as each is based on different underlying assumptions.

\subsubsection{Equation of state}

The equation of state approach was conceptualized by the French chemist Marcellin Berthelot ${ }^{\dagger \dagger}$ in the late 1800s and describes $\gamma_{\mathrm{sl}}$ as being dependent on properties of both the solid and liquid. ${ }^{148}$ A consequence of this approach is that surface tension is evaluated as a single parameter and can not be dissociated into individual components:

$$
F=\left(\gamma_{s}, \gamma_{l}, \gamma_{s l}\right)=0 \quad \text { Equation } 6
$$

Berthelot postulated that the work of interfacial adhesion $\left(\mathrm{W}_{\mathrm{sl}}\right)$ is equal to the geometric mean of the work of solid cohesion $\left(\mathrm{W}_{\mathrm{s}}\right)$ and the work of liquid cohesion $\left(\mathrm{W}_{\mathrm{l}}\right)$ :

$$
W_{s l}=\sqrt{W_{s} W_{l}} \quad \text { Equation } 7
$$

French chemists Anthanase Dupré and Paul Dupré introduced the Dupré equation in 1869 which relates $\mathrm{W}_{\mathrm{sl}}$ and surface tension: ${ }^{149}$

"T Marcellin Berthelot was a prominent French chemist during the middle and late 1800s. He conducted many experiments investigating the concepts of chemistry and the relationships between work, heat, and chemical reactions. He developed many theories explaining the state of chemical reactions during a time when not much about chemistry was properly understood. He was awarded the Copley Medal in 1900 "for his brilliant services to chemical science" and is considered to be one of the greatest chemists of all time. ${ }^{147}$ 


$$
W_{s l}=\gamma_{s}+\gamma_{l}-\gamma_{s l} \quad \text { Equation } 8
$$

Berthelot then combined his equation (Equation 7) with the Dupré equation (Equation 8) to obtain what became known as the Berthelot hypothesis:

$$
\gamma_{s l}=\gamma_{s}+\gamma_{l}-2 \sqrt{\gamma_{s} \gamma_{l}} \quad \text { Equation } 9
$$

The Berthelot hypothesis is the basis for equation of state models. Agnes Pockels ${ }^{\star}$ deduced the Young-Dupré equation in 1914 by combining the Young and Dupré equations: ${ }^{153-155}$

$$
W=\gamma_{l}(1+\cos \theta) \quad \text { Equation } 10
$$

In 1957, Girifalco and Good redeveloped the Dupré equation by including a parameter describing interfacial interactions $(\Phi):^{156}$

* Agnes Pockels was born in Venice, Italy in 1862 to native German parents. The family moved to Brunswick, Germany in 1871 where Agnes would live, take care of her parents, study alongside her younger brother, Fritz, and conduct experiments in her home. Pockels is notable for her contributions to surface science when the field was just emerging in the last 1800s. Agnes had an "enthusiastic interest in the natural sciences, especially physics" in high school but was unable to formally continue her education because she was female. Fortunately, her brother Fritz also enjoyed physics and became a professor of theoretical physics at the University of Heidelberg. He provided Agnes with German literature on surface science and she conducted her own experiments at home. "What millions of women see every day without pleasure and are anxious to clean away, i.e., the greasy washing-up water, encouraged this girl to make observations and eventually to...scientific investigation," wrote her sister-in-law. ${ }^{150,151}$

Agnes Pockels wrote about her observations and quantitative analysis to Lord Rayleigh, a prominent English physicist. Rayleigh then wrote Nature and Pockel's work was first published in 1891 . $^{152}$ She is ultimately credited with 15 publications from 1891 to 1933. She invented the original design for what would become the Langmuir trough designed by Irving Langmuir. An interesting connection, Agnes Pockels translated a textbook by George Howard Darwin, the son of Charles Darwin and father of Charles Galton (C.G.) Darwin (subject of another footnote on page 3$){ }^{150,151}$ 


$$
\gamma_{s l}=\gamma_{s}+\gamma_{l}-2 \Phi \sqrt{\gamma_{s} \gamma_{l}} \quad \text { Equation } 11
$$

where $\Phi=1$ when interactions of the same type occur (thus, reducing to the Berthelot hypothesis).

\subsubsection{Neumann model}

A.W. Neumann et al. developed the Neumann model in 1974 which uses equation of state theory to describe the contact angle of a liquid on a solid surface: ${ }^{138}$

$$
\cos \theta=-1+2 \sqrt{\frac{\gamma_{s}}{\gamma_{l}}} e^{-\beta\left(\gamma_{s}-\gamma_{l}\right)^{2}}
$$

Equation 12

where $\gamma_{s}$ is the solid surface energy, $\gamma_{1}$ is the liquid surface tension, and $\beta$ is a parameter related to the solid surface. Rearrangement of Equation 12 results in:

$$
\ln \left[\gamma_{l}\left(\frac{1+\cos \theta}{2}\right)^{2}\right]=-2 \beta\left(\gamma_{s}-\gamma_{l}\right)^{2}+\ln \left(\gamma_{s}\right) \quad \text { Equation } 13
$$

The liquid surface energy is known and plotting the left side of Equation 13 against $\gamma_{1}$ will produce a parabolic curve. A second-order polynomial regression of the plotted data allows for determination of $\beta$ and $\gamma_{s}$.

Neumann's equation is by far the most common equation of state method for evaluating surface energy from contact angles. The salient drawback is the ambiguity surrounding $\beta$. There is no consensus on the meaning of $\beta$ and it has been postulated to be either a material specific 
constant or simply a fitting parameter with no physical meaning. ${ }^{137}$ For this study, $\beta$ was allowed to be fit and there was no discernable correlation between $\beta$ and the raw data (see Section 3.7 on page 45$)$.

The Neumann equation was empirically derived by an iterative process using contact angle data on various polymers. ${ }^{137,157,158}$ Previous researchers have reported significant differences between the Neumann and Owens-Wendt models when surface free energy is 20-50 $\mathrm{mJ} / \mathrm{m}^{2}$, a range encompassing myriad common materials. ${ }^{125,137,159-161}$

\subsubsection{Dissociation into components}

Alternative to the equation of state approach, surface energy can be considered the sum of many individual and independent components, each associated with specific interfacial interactions:

$$
\gamma_{s}=\gamma_{s}^{d}+\gamma_{s}^{p}+\gamma_{s}^{h}+\gamma_{s}^{i}+\gamma_{s}^{a b}+\gamma_{s}^{\circ} \quad \text { Equation } 14
$$

where $\gamma_{s}^{\mathrm{d}}, \gamma_{\mathrm{s}}^{\mathrm{p}}, \gamma_{\mathrm{s}}^{\mathrm{h}}, \gamma_{\mathrm{s}}^{\mathrm{i}}, \gamma_{\mathrm{s}}^{\mathrm{ab}}$, and $\gamma_{\mathrm{s}}^{\mathrm{o}}$ refer to the dispersive, polar, hydrogen bonding, induction, acid-base, and all remaining components, respectively. This approach was first reported by Frederick Fowkes in 1963. Fowkes originally focused only on the dispersive component $\left(\gamma_{\mathrm{s}}{ }^{\mathrm{d}}\right)$ and developed the Berthelot hypothesis (Equation 9) for dispersive (London) forces: ${ }^{139,}$ 162-165

$$
\gamma_{s l}=\gamma_{s}+\gamma_{l}-2 \sqrt{\gamma_{s}^{d} \gamma_{l}^{d}} \quad \text { Equation } 15
$$


Fowkes then simplified Equation 14 to formulate the Fowkes model which considers only the dispersive $\left(\gamma_{s}{ }^{d}\right)$ and polar $\left(\gamma_{s}^{p}\right)$ components and assumes all others are negligible. ${ }^{139}$ Della Volpe et al. recently made an interesting case in support of the multicomponent approach. ${ }^{166,167}$

\subsubsection{Fowkes model}

Fowkes surface energy theory combines the Young and Young-Dupree equation (Equation 5 and Equation 10) and dissociates liquid and solid surface energy into its polar and non-polar (dispersive) components: ${ }^{139}$

$$
\begin{array}{cc}
\frac{\gamma_{l}(1+\cos \theta)}{2}=\left(\gamma_{l}^{d}\right)^{1 / 2}\left(\gamma_{s}^{d}\right)^{1 / 2}+\left(\gamma_{l}^{p}\right)^{1 / 2}\left(\gamma_{s}^{p}\right)^{1 / 2} & \text { Equation } 16 \\
\gamma_{s}=\gamma_{s}^{d}+\gamma_{s}^{p} & \text { Equation } 17
\end{array}
$$

where $\gamma_{1}^{\mathrm{d}}$ and $\gamma_{1}^{\mathrm{p}}$ are the liquid dispersive and polar components, respectively, and $\gamma_{\mathrm{s}}^{\mathrm{d}}$ and $\gamma_{\mathrm{s}}^{\mathrm{p}}$ are the solid dispersive and polar components, respectively. First, a nonpolar aprotic liquid, e.g., diiodomethane $\left(\gamma_{1}^{\mathrm{p}}=0\right)$, is tested and the dispersive component of the solid surface energy, $\gamma_{s}{ }^{d}$, is computed from Equation 16. Second, a polar protic liquid, e.g., water, is tested and using $\gamma_{s}{ }^{d}$ and Equation 16 the polar component of the solid surface energy, $\gamma_{\mathrm{s}}^{\mathrm{p}}$, is computed.

Fowkes theory assumes that (1) polar and dispersive components are independent of each other, (2) the other components $\left(\gamma_{s}{ }^{\mathrm{h}}, \gamma_{s}{ }^{\mathrm{i}}, \gamma_{\mathrm{s}}^{\mathrm{ab}}\right.$, and $\left.\gamma_{\mathrm{s}}{ }^{\mathrm{o}}\right)$ of Equation 14 are negligible, and (3) the surface energy terms are additive and do not interact in any other way (Equation 17). Generally, these assumptions hold up to scientific reasoning and are applicable to most surfaces encountered. ${ }^{137,139,165}$ 


\subsubsection{Owens-Wendt model}

Owens and Wendt assumed that all components in Equation 14, aside from the dispersive part $\left(\gamma_{s}{ }^{d}\right)$, were part of the polar component $\left(\gamma_{s}^{p}\right)$. Thus, Equation 14 is reduced to: ${ }^{129}$

$$
\gamma_{s}=\gamma_{s}^{d}+\gamma_{s}^{p} \quad \text { Equation } 18
$$

where $\gamma_{s}^{\mathrm{p}}=\gamma_{\mathrm{s}}^{\mathrm{p}}\left(\gamma_{\mathrm{s}}^{\mathrm{p}}, \gamma_{\mathrm{s}}^{\mathrm{h}}, \gamma_{\mathrm{s}}^{\mathrm{i}}, \gamma_{\mathrm{s}}^{\mathrm{ab}}, \gamma_{\mathrm{s}}^{\mathrm{o}}\right)$. This is fundamentally different from Fowkes' assumption that $\gamma_{s}{ }^{\mathrm{h}}, \gamma_{s}^{\mathrm{i}}, \gamma_{\mathrm{s}}^{\mathrm{ab}}$, and $\gamma_{\mathrm{s}}^{\mathrm{o}}$ are negligible. Owens and Wendt generalized Equation 15 to account for both polar and dispersive forces: ${ }^{129,137}$

$$
\gamma_{s l}=\gamma_{s}+\gamma_{l}-2 \sqrt{\gamma_{s}^{d} \gamma_{l}^{d}}-2 \sqrt{\gamma_{s}^{p} \gamma_{l}^{p}} \quad \text { Equation } 19
$$

Combining Young's equation (Equation 5) with Equation 19 yields the Owens-Wendt equation:

$$
1+\cos \theta=2 \sqrt{\gamma_{s}^{d}}\left(\frac{\sqrt{\gamma_{l}^{d}}}{\gamma_{l}}\right)+2 \sqrt{\gamma_{s}^{p}}\left(\frac{\sqrt{\gamma_{l}^{p}}}{\gamma_{l}}\right)
$$

Rearranging Equation 20 provides a graphical solution that requires at least two test liquids:

$$
\frac{\gamma_{l}(\cos \theta+1)}{2\left(\gamma_{l}^{d}\right)^{1 / 2}}=\left(\gamma_{s}^{p}\right)^{1 / 2} \frac{\left(\gamma_{l}^{p}\right)^{1 / 2}}{\left(\gamma_{l}^{d}\right)^{1 / 2}}+\left(\gamma_{s}^{d}\right)^{1 / 2}
$$

Equation 21 
Plotting the left side of Equation 21 against $\left(\gamma_{1}^{\mathrm{p}}\right)^{1 / 2} /\left(\gamma_{1}^{\mathrm{d}}\right)^{1 / 2}$ will produce a linear line and regression of the data allows for determination of $\gamma_{s}{ }^{p}$ as the square of the slope and $\gamma_{s}{ }^{d}$ as the square of the y-intercept. As mentioned previously, better fitting results are obtained when more than two liquids are used and the liquid surface tensions vary significantly. ${ }^{129,137}$

\subsubsection{Remarks on the surface energy models}

The equation of state and component theories of surface energy are based on fundamentally different concepts. The equation of state theory assumes that the interfacial solid-liquid surface energy $\left(\gamma_{\mathrm{sl}}\right)$ is dependent of both solid and liquid properties; whereas, the component theory assumes that the solid and liquid properties are independent and surface energy components can be dissociated into separate contributions which are additive. Both methods have scientific basis and researchers have concomitantly used both to describe solid surface free energy. Robust results are necessary for comparing data of different researchers; therefore, four test liquids with a wide range of surface tension properties were chosen for this investigation.

\subsection{GRAPHENE CHARACTERIZATION}

Figure 8 shows the Raman spectrum of $\mathrm{G} / \mathrm{Cu}$ immediately after synthesis. The intensity of disorder-induced Raman D-peak at $1350 \mathrm{~cm}^{-1}$ is very low, indicating that the graphene film has very few defects. The peaks at 1595 and $2695 \mathrm{~cm}^{-1}$ are identified as $\mathrm{G}$ and $2 \mathrm{D}$ peaks, respectively, and their presence agrees well with previous $\mathrm{G} / \mathrm{Cu}$ spectra. ${ }^{168,}{ }^{169}$ The $2 \mathrm{D}$ peak shows a sharp single Lorentzian profile, which is a clear indication of monolayer graphene. ${ }^{168}$ 
SEM and AFM data show that the surface of $\mathrm{G} / \mathrm{Cu}$ is rough due to the polycrystalline copper foil substrate; however, no apparent particulate contaminant was observed (see Supporting Info of Reference 75). In a previous study, $\mathrm{G} / \mathrm{Cu}$ samples prepared using the same protocol as we used here showed a minimum graphene coverage of $>99.9 \%$ on $\mathrm{Cu}$ foil. ${ }^{77}$

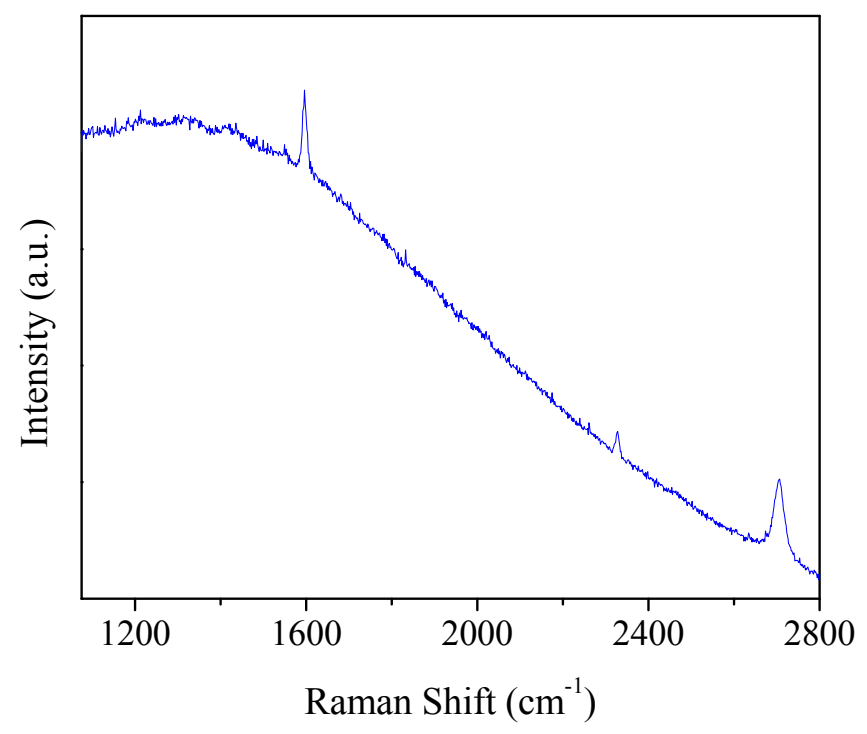

Figure 8. Raman spectrum of graphene on copper $(\mathrm{G} / \mathrm{Cu})$. The small peak at $2330 \mathrm{~cm}^{-1}$ is due to atmospheric $\mathrm{N}_{2} .{ }^{170}$ 


\subsection{CONTACT ANGLE AND SURFACE ENERGY}

Contact angle data on $\mathrm{G} / \mathrm{Cu}$ for the four test liquids is plotted in Figure 9. Average values for $\mathrm{G} / \mathrm{Cu}$ and HOPG are shown in Table 3 and Table 4, respectively. Figure 10 shows the polynomial fitting of data by Neumann model and Figure 11 shows the linear fitting of data by Owens-Wendt (extended Fowkes) model for $\mathrm{G} / \mathrm{Cu}$. Table 5 lists the calculated total surface energy and $\beta$ parameter for the Neumann model and the total surface energy and its polar and dispersive components for the Fowkes and Owens-Wendt models. Table 6 lists surface energy results on HOPG.

The surface energy of $\mathrm{G} / \mathrm{Cu}$ is highest immediately after synthesis and decreases with exposure time in air. The surface energy of fresh $\mathrm{G} / \mathrm{Cu}$ tested within 30 seconds of being removed from the CVD chamber was $53.0 \pm 4.3 \mathrm{~mJ} / \mathrm{m}^{2}$ (Owens-Wendt), $62.2 \pm 3.1 \mathrm{~mJ} / \mathrm{m}^{2}$ (Fowkes), and $63.8 \pm 2.0 \mathrm{~mJ} / \mathrm{m}^{2}$ (Neumann). The surface energy decreased after aging in ambient air for $1 \mathrm{~h}$ and further decreased after $2 \mathrm{~h}$ of aging. G/Cu exposed to ambient air for 24 $\mathrm{h}$ showed the lowest surface energy of $37.5 \pm 2.3 \mathrm{~mJ} / \mathrm{m}^{2}$ (Owens-Wendt), $45.6 \pm 3.9 \mathrm{~mJ} / \mathrm{m}^{2}$ (Fowkes), and $57.4 \pm 2.1 \mathrm{~mJ} / \mathrm{m}^{2}$ (Neumann). According to results from Owens-Wendt model, the polar component of surface energy $\left(\gamma_{\mathrm{s}}^{\mathrm{p}}\right)$ is the greatest on fresh $\mathrm{G} / \mathrm{Cu}$ and decreases by $75 \%$ after

$24 \mathrm{~h}$ exposure to air while the dispersive component $\left(\gamma_{\mathrm{s}}{ }^{\mathrm{d}}\right)$ decreases $13 \%$. The results from Fowkes model show a similar trend, indicating that $\mathrm{G} / \mathrm{Cu}$ surface is initially mildly polar and becomes increasingly non-polar upon exposure to air. Similar trends are seen for HOPG. 

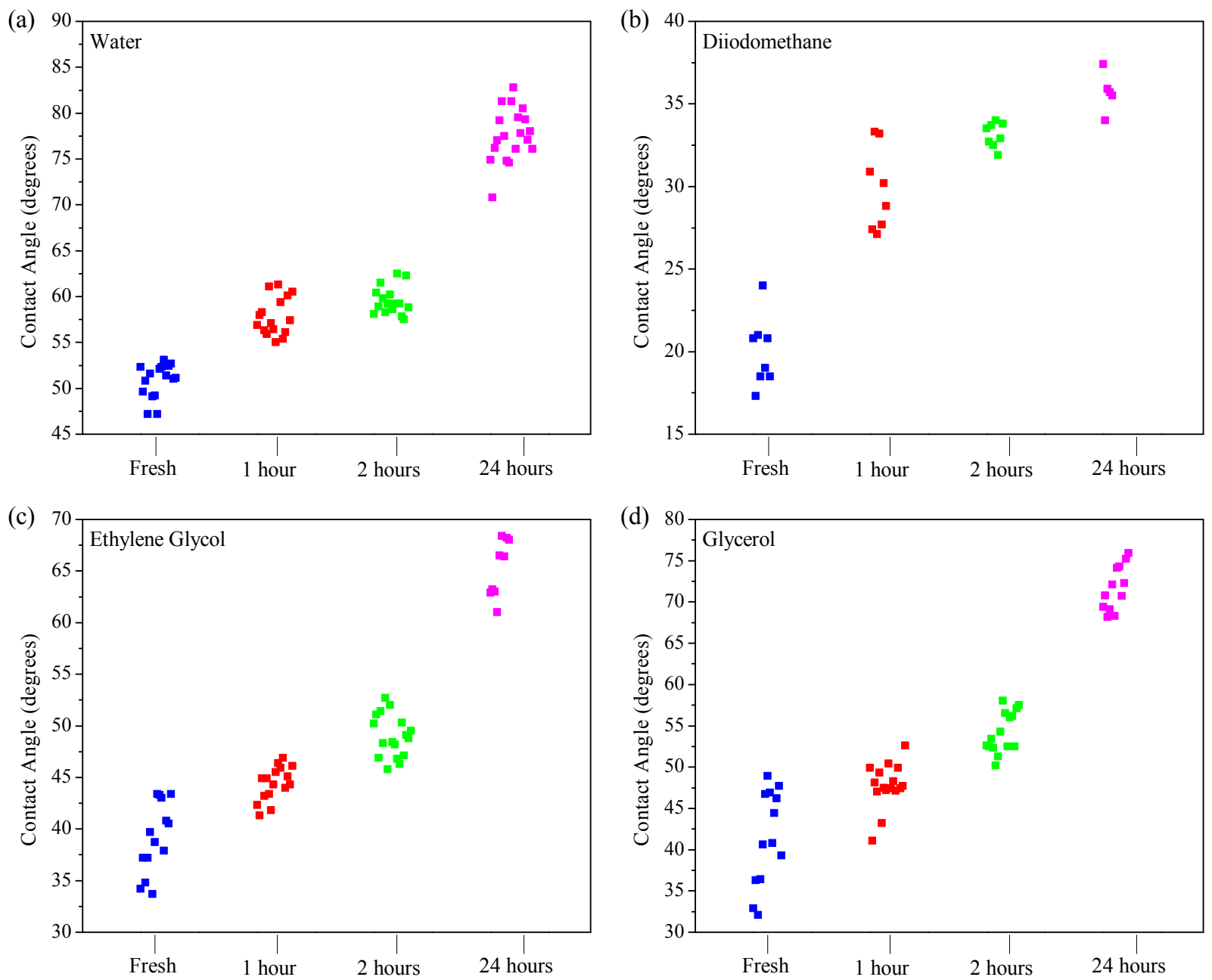

Figure 9. Contact angle data of $\mathrm{G} / \mathrm{Cu}$ for (a) water, (b) diiodomethane, (c) ethylene glycol, and (d) glycerol. All data were taken at the time indicated on the x-axis and are shifted horizontally in the figures for clarity. 
Table 3. Contact angle data on $\mathrm{G} / \mathrm{Cu}$ presented as average (standard deviation) of tests for water, diiodomethane, ethylene glycol, and glycerol.

\begin{tabular}{|c|c|c|c|c|}
\cline { 2 - 5 } \multicolumn{1}{c|}{} & Water & Diiodomethane & $\begin{array}{c}\text { Ethylene } \\
\text { glycol }\end{array}$ & Glycerol \\
\hline Fresh & $50.8(1.9)$ & $20.0(2.1)$ & $38.8(3.5)$ & $40.4(5.8)$ \\
1h air & $57.8(2.1)$ & $29.8(2.5)$ & $44.4(1.7)$ & $47.8(2.7)$ \\
2h air & $59.5(1.5)$ & $33.1(0.7)$ & $49.0(2.1)$ & $54.2(2.5)$ \\
24h air & $77.6(2.9)$ & $35.7(1.2)$ & $65.0(2.8)$ & $71.7(2.7)$ \\
\hline
\end{tabular}

Table 4. Contact angle data on SPI-2 HOPG presented as average (standard deviation) of tests for water, diiodomethane, ethylene glycol, and glycerol.

\begin{tabular}{|c|c|c|c|c|}
\cline { 2 - 5 } \multicolumn{1}{c|}{} & Water & Diiodomethane & $\begin{array}{c}\text { Ethylene } \\
\text { glycol }\end{array}$ & Glycerol \\
\hline Fresh & $64.63(2.89)$ & $20.60(1.55)$ & $43.25(1.41)$ & $35.75(0.57)$ \\
1h air & $89.09(1.39)$ & $24.75(5.09)$ & $44.15(0.14)$ & $51.95(0.78)$ \\
24h air & $97.01(1.81)$ & $38.15(1.06)$ & $60.25(5.87)$ & $74.45(4.88)$ \\
\hline
\end{tabular}



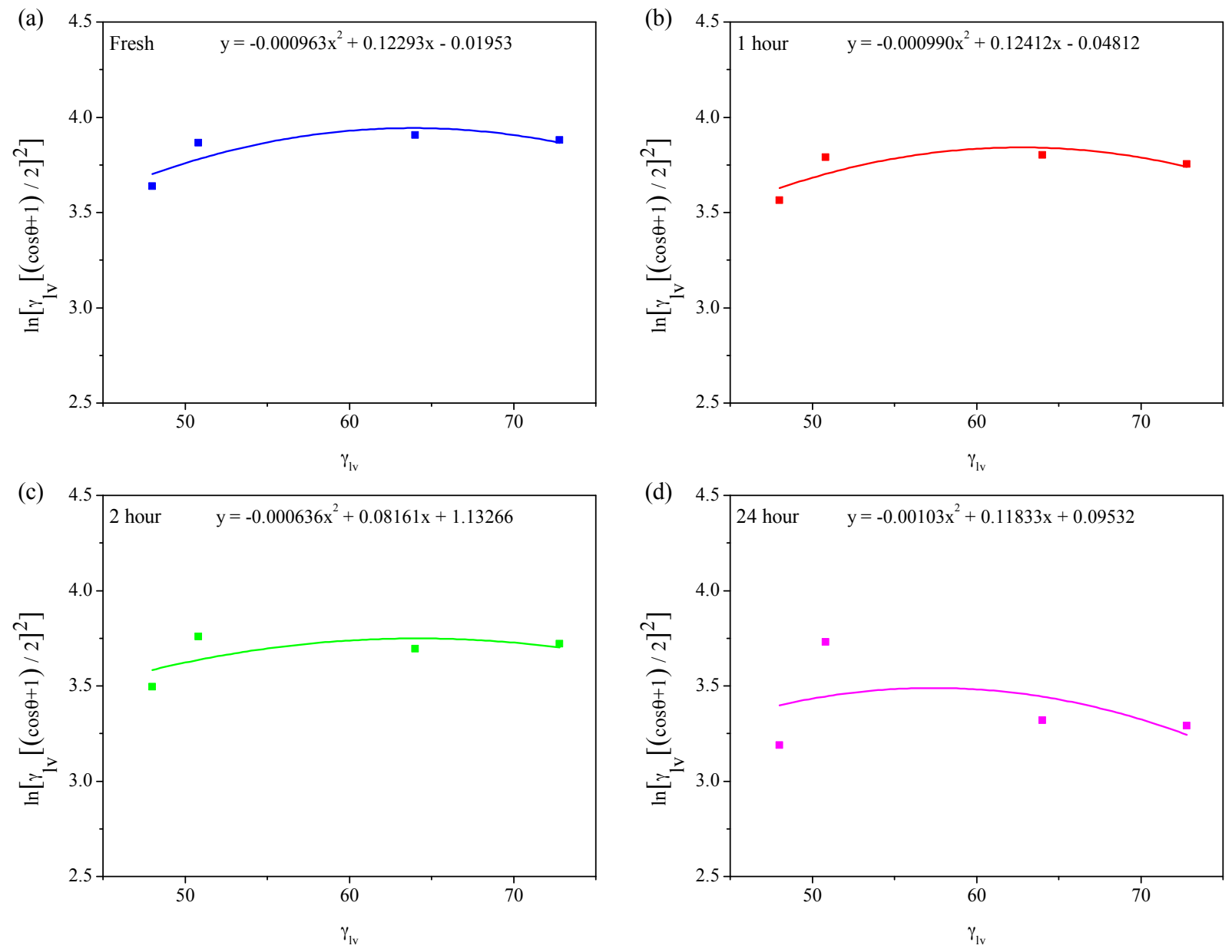

Figure 10. Neumann model surface energy plots of G/Cu. (a) Fresh, (b) 1 hour aged, (c) 2 hour aged, and (d) 24 hour aged $\mathrm{G} / \mathrm{Cu}$. 

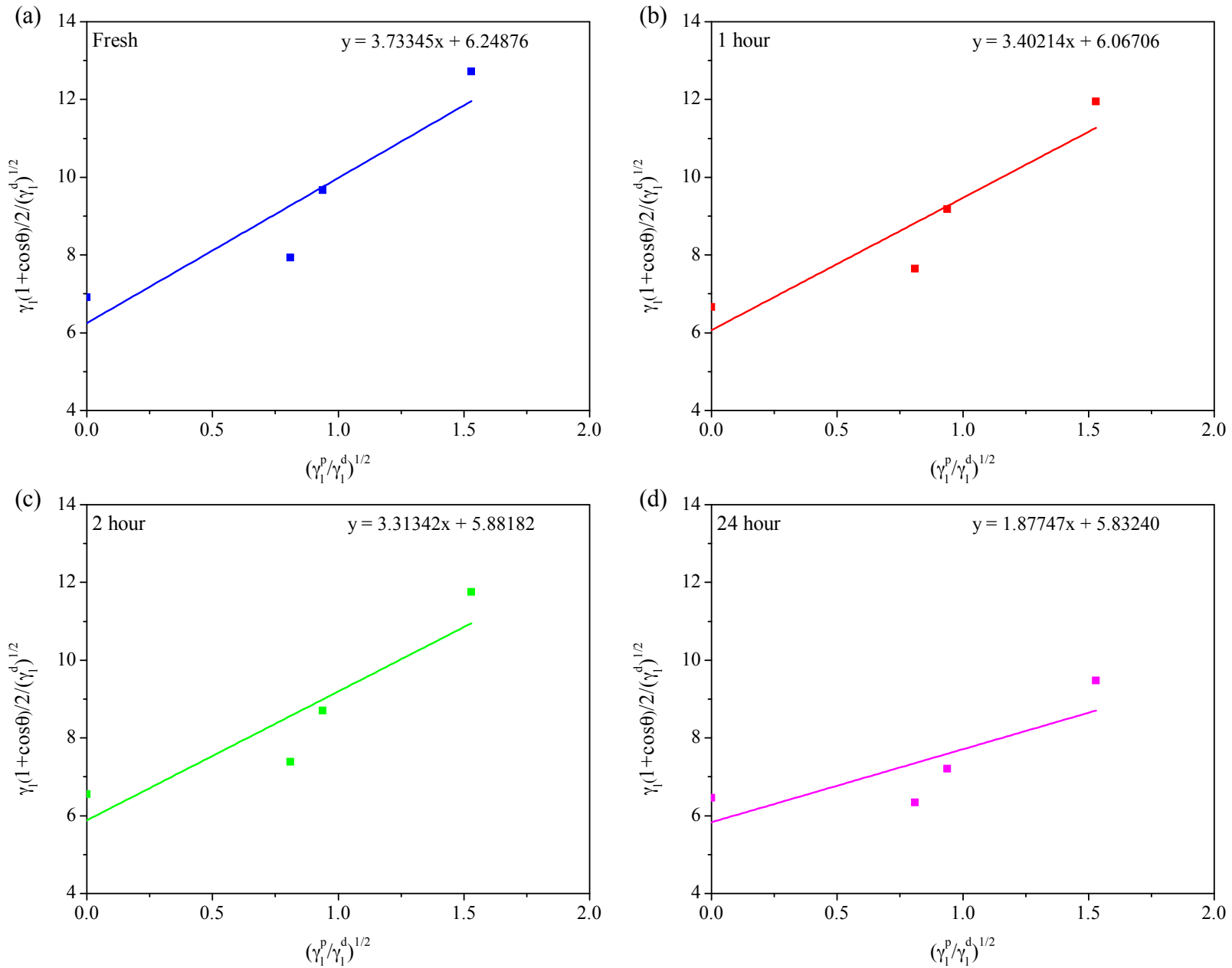

Figure 11. Owens-Wendt (extended Fowkes) surface energy plots of G/Cu. (a) Fresh, (b) 1 hour aged, (c) 2 hour aged, and (d) 24 hour aged G/Cu. 
Table 5. Surface free energy calculation results of G/Cu determined by Neumann, Fowkes, and Owens-Wendt models. Data presented as average (standard deviation).

\begin{tabular}{|c|c|c|c|c|c|c|c|c|}
\hline \multirow[b]{2}{*}{$\mathrm{G} / \mathrm{Cu}$} & \multicolumn{2}{|c|}{ Neumann } & \multicolumn{3}{|c|}{ Fowkes } & \multicolumn{3}{|c|}{ Owens-Wendt } \\
\hline & $\beta$ & $\begin{array}{c}\gamma_{\mathrm{s}} \\
\left(\mathrm{mJ} / \mathrm{m}^{2}\right)\end{array}$ & $\begin{array}{c}\gamma_{\mathrm{s}}^{\mathrm{p}} \\
\left(\mathrm{mJ} / \mathrm{m}^{2}\right)\end{array}$ & $\begin{array}{c}\gamma_{\mathrm{s}}^{\mathrm{d}} \\
\left(\mathrm{mJ} / \mathrm{m}^{2}\right)\end{array}$ & $\begin{array}{c}\gamma_{\mathrm{s}} \\
\left(\mathrm{mJ} / \mathrm{m}^{2}\right)\end{array}$ & $\begin{array}{c}\gamma_{\mathrm{s}}^{\mathrm{p}} \\
\left(\mathrm{mJ} / \mathrm{m}^{2}\right)\end{array}$ & $\begin{array}{c}\gamma_{\mathrm{s}}^{\mathrm{d}} \\
\left(\mathrm{mJ} / \mathrm{m}^{2}\right)\end{array}$ & $\begin{array}{c}\gamma_{\mathrm{s}} \\
\left(\mathrm{mJ} / \mathrm{m}^{2}\right)\end{array}$ \\
\hline Fresh & $\begin{array}{c}0.00048138 \\
(0.00023229)\end{array}$ & $\begin{array}{l}63.8 \\
(2.0)\end{array}$ & $\begin{array}{l}14.4 \\
(1.7)\end{array}$ & $\begin{array}{l}47.8 \\
(1.4)\end{array}$ & $\begin{array}{r}62.2 \\
(3.1)\end{array}$ & $\begin{array}{l}13.9 \\
(2.0)\end{array}$ & $\begin{array}{l}39.1 \\
(2.4)\end{array}$ & $\begin{array}{l}53.0 \\
(4.3)\end{array}$ \\
\hline 1h aged & $\begin{array}{c}0.00049506 \\
(0.00014586)\end{array}$ & $\begin{array}{r}62.7 \\
(0.4)\end{array}$ & $\begin{array}{l}12.0 \\
(1.7)\end{array}$ & $\begin{array}{l}44.3 \\
(1.8)\end{array}$ & $\begin{array}{l}56.3 \\
(3.5)\end{array}$ & $\begin{array}{l}11.6 \\
(1.8)\end{array}$ & $\begin{array}{l}36.8 \\
(1.7)\end{array}$ & $\begin{array}{l}48.4 \\
(3.5)\end{array}$ \\
\hline $2 \mathrm{~h}$ aged & $\begin{array}{c}0.00031820 \\
(0.00004533)\end{array}$ & $\begin{array}{l}64.1 \\
(0.5)\end{array}$ & $\begin{array}{l}11.6 \\
(1.6)\end{array}$ & $\begin{array}{l}42.9 \\
(0.7)\end{array}$ & $\begin{array}{l}54.5 \\
(2.3)\end{array}$ & $\begin{array}{l}11.0 \\
(1.7)\end{array}$ & $\begin{array}{l}34.6 \\
(1.0)\end{array}$ & $\begin{array}{l}45.6 \\
(2.7)\end{array}$ \\
\hline $24 \mathrm{~h}$ aged & $\begin{array}{c}0.00051500 \\
(0.00023892)\end{array}$ & $\begin{array}{l}57.4 \\
(2.1)\end{array}$ & $\begin{array}{l}3.9 \\
(2.8)\end{array}$ & $\begin{array}{l}41.7 \\
(1.1)\end{array}$ & $\begin{array}{l}45.6 \\
(3.9)\end{array}$ & $\begin{array}{l}3.5 \\
(2.5)\end{array}$ & $\begin{array}{l}34.0 \\
(0.2)\end{array}$ & $\begin{array}{l}37.5 \\
(2.3)\end{array}$ \\
\hline
\end{tabular}

Table 6. Surface free energy calculation results of SPI-2 HOPG determined by Neumann, Fowkes, and Owens-Wendt models. Data presented as average (standard deviation).

\begin{tabular}{|c|c|c|c|c|c|c|c|c|}
\hline \multirow[b]{2}{*}{ HOPG } & \multicolumn{2}{|c|}{ Neumann } & \multicolumn{3}{|c|}{ Fowkes } & \multicolumn{3}{|c|}{ Owens-Wendt } \\
\hline & $\beta$ & $\begin{array}{c}\gamma_{\mathrm{s}} \\
\left(\mathrm{mJ} / \mathrm{m}^{2}\right)\end{array}$ & $\begin{array}{c}\gamma_{\mathrm{s}}^{\mathrm{p}} \\
\left(\mathrm{mJ} / \mathrm{m}^{2}\right)\end{array}$ & $\begin{array}{c}\gamma_{\mathrm{s}}^{\mathrm{d}} \\
\left(\mathrm{mJ} / \mathrm{m}^{2}\right)\end{array}$ & $\begin{array}{c}\gamma_{\mathrm{s}} \\
\left(\mathrm{mJ} / \mathrm{m}^{2}\right)\end{array}$ & $\begin{array}{c}\gamma_{\mathrm{s}}^{\mathrm{p}} \\
\left(\mathrm{mJ} / \mathrm{m}^{2}\right)\end{array}$ & $\begin{array}{c}\gamma_{\mathrm{s}}^{\mathrm{d}} \\
\left(\mathrm{mJ} / \mathrm{m}^{2}\right)\end{array}$ & $\begin{array}{c}\gamma_{\mathrm{s}} \\
\left(\mathrm{mJ} / \mathrm{m}^{2}\right)\end{array}$ \\
\hline Fresh & $\begin{array}{c}0.00133270 \\
(0.00029094)\end{array}$ & $\begin{array}{l}60.23 \\
(0.86)\end{array}$ & $\begin{array}{c}7.79 \\
(2.69)\end{array}$ & $\begin{array}{l}47.60 \\
(0.47)\end{array}$ & $\begin{array}{l}55.40 \\
(3.16)\end{array}$ & $\begin{array}{c}8.10 \\
(2.49)\end{array}$ & $\begin{array}{l}43.50 \\
(1.62)\end{array}$ & $\begin{array}{l}51.60 \\
(0.87)\end{array}$ \\
\hline $1 \mathrm{~h}$ aged & $\begin{array}{c}0.00209292 \\
(0.00011710)\end{array}$ & $\begin{array}{l}\mathbf{5 7 . 1 3} \\
(\mathbf{0 . 0 9 )}\end{array}$ & $\begin{array}{c}0.54 \\
(0.17)\end{array}$ & $\begin{array}{l}46.24 \\
(1.57)\end{array}$ & $\begin{array}{l}46.78 \\
(1.75)\end{array}$ & $\begin{array}{c}0.76 \\
(0.18)\end{array}$ & $\begin{array}{l}50.40 \\
(0.41)\end{array}$ & $\begin{array}{l}51.15 \\
(0.60)\end{array}$ \\
\hline $24 \mathrm{~h}$ aged & $\begin{array}{c}0.00152093 \\
(0.00005682)\end{array}$ & $\begin{array}{l}55.00 \\
(0.25)\end{array}$ & $\begin{array}{c}0.10 \\
(0.14)\end{array}$ & $\begin{array}{l}40.53 \\
(0.52)\end{array}$ & $\begin{array}{l}40.63 \\
(0.66)\end{array}$ & $\begin{array}{c}0.12 \\
(0.17)\end{array}$ & $\begin{array}{l}41.33 \\
(1.33)\end{array}$ & $\begin{array}{l}41.45 \\
(1.50)\end{array}$ \\
\hline
\end{tabular}




\subsection{ELLIPSOMETRY CHARACTERIZATION}

Figure 12 shows the evolution of phase shift on freshly-synthesized $\mathrm{G} / \mathrm{Cu}$ surface as a function of air exposure time. The data was collected from the same spot on the G/Cu surface. All the $\mathrm{x}$ values are shifted by 5 minutes to reflect transfer time of the sample from CVD chamber to ellipsometer stage. The increasing trend of phase shift shows clear evidence that a thin layer of adsorbate formed on $\mathrm{G} / \mathrm{Cu}$ surface during the first $2 \mathrm{~h}$ of air exposure. Interestingly, our previous work demonstrated a similar WCA increasing trend on fresh $\mathrm{G} / \mathrm{Cu}$ surface due to airborne hydrocarbon contamination, ${ }^{77}$ further corroborating that the phase shift increase is induced by the adsorption of airborne hydrocarbons.
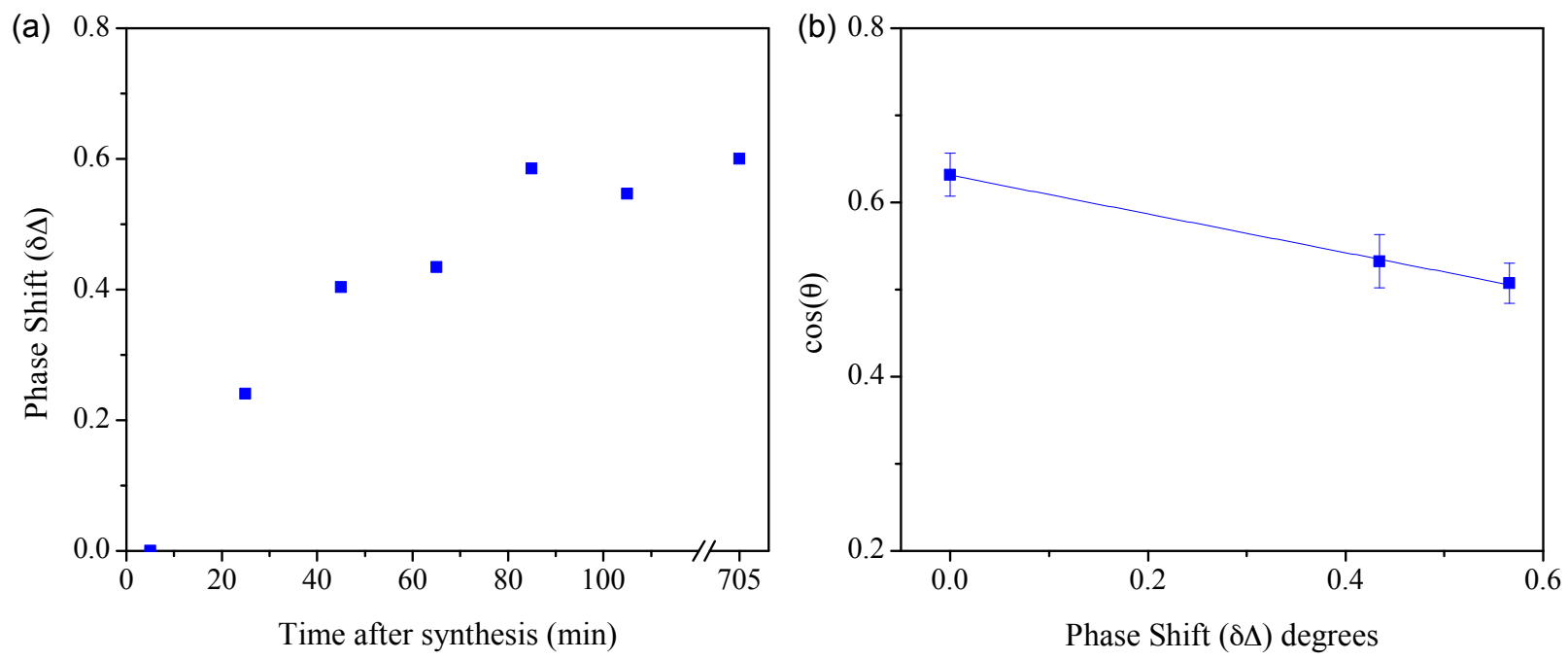

Figure 12. Ellipsometry correlations of G/Cu. (a) Phase shift $(\delta \Delta)$ as a function of air exposure and (b) correlation between WCA $(\theta)$ and ellipsometry phase shift on fresh, $1 \mathrm{~h}$ aged, and $2 \mathrm{~h}$ aged $\mathrm{G} / \mathrm{Cu}$. Accuracy of the phase shift $(\Delta)$ is $0.1 \% .{ }^{171}$ The solid line is a linear fit of the data: $\mathrm{y}=-0.222 * \mathrm{x}+0.631$ and $\mathrm{r}^{2}=0.99$. 


\subsection{EFFECT OF SURFACE ROUGHNESS}

The Wenzel equation allows comparison of the experimentally determined apparent $\mathrm{CA}\left(\theta_{\mathrm{app}}\right)$ and the Young's CA $\left(\theta_{\mathrm{Y}}\right)$ which is the contact angle determined on a smooth surface: ${ }^{172}$

$$
\cos \theta_{a p p}=r \cos \theta_{Y} \quad \text { Equation } 22
$$

$$
r=\frac{A_{a}}{A_{p}} \quad \text { Equation } 23
$$

where $r$ is defined by the ratio of apparent surface area to projected surface area. The $r$ value for our sample was determined by AFM to be in the range of 1.00095-1.0257 depending on scanning parameters. ${ }^{173,} 174$ Using the $\mathrm{r}$ value upper bound of 1.0257 and $50.9^{\circ}$ measured WCA, the corresponding $\theta_{\mathrm{Y}}$ value was found to be $52.0^{\circ}$ for fresh $\mathrm{G} / \mathrm{Cu}$. The difference between $\theta_{\text {app }}$ and $\theta_{\mathrm{Y}}$ in this case is only $1.1^{\circ}$. Noting that the uncertainty of WCA test is at least $\pm 1^{\circ}$, we conclude that the error introduced by surface roughness is negligible on our $\mathrm{G} / \mathrm{Cu}$ samples.

In addition to the above analysis, we also prepared monolayer graphene on ultra-flat $\mathrm{Cu}$ substrates (flat $\mathrm{G} / \mathrm{Cu} ; r=1.00019$ ) following a published procedure. ${ }^{175}$ The WCA of fresh flat $\mathrm{G} / \mathrm{Cu}$ was $56.3^{\circ} \pm 3.9^{\circ}$ and increased to $81.2^{\circ} \pm 1.4^{\circ}$ after $24 \mathrm{~h}$ of air exposure. The WCA on flat $\mathrm{G} / \mathrm{Cu}$ is about $5^{\circ}$ greater than that on $\mathrm{G} / \mathrm{Cu}$ foil. Unfortunately, because the two types of samples were prepared and measured in different buildings, it is likely that local air quality contributes to the difference in the WCA; ${ }^{176}$ the $5^{\circ}$ difference should be regarded as the upper limit of the roughness effect. 


\subsection{TIME DEPENDENCE}

The surface energy calculated by the Fowkes model matches the Neumann results very well on fresh graphene. The difference between the two models on fresh and $24 \mathrm{~h}$ aged $\mathrm{G} / \mathrm{Cu}$ is $3 \%$ and $21 \%$, respectively, and the Neumann model yields a higher surface energy. The Owens-Wendt model extends upon the Fowkes model, makes different assumptions, and utilizes four test liquids with different surface energies; therefore, the Owens-Wendt model is expected to be less dependent upon chosen test liquids. On fresh and $24 \mathrm{~h}$ aged $\mathrm{G} / \mathrm{Cu}$, the difference between the Fowkes and Owens-Wendt models are $15 \%$ and $18 \%$, respectively, and the Fowkes model yields a higher surface energy. Of all three models, Neumann's provides the greatest $\gamma_{\mathrm{s}}$ and OwensWendt's provides the lowest $\gamma_{\mathrm{s}}$ for $\mathrm{G} / \mathrm{Cu}$ and (generally) HOPG.

The Neumann approach is based on equation of state theory and introduces the surface dependent $\beta$ parameter. Our calculations indicate that the Neumann surface energy is consistently higher than the Fowkes and Owens-Wendt models, although the Neumann and Fowkes results are similar on fresh $\mathrm{G} / \mathrm{Cu}$. It has been proposed that $\beta=0.0001247$ can be expressed as a universal constant that is independent of test solid. ${ }^{134}$ Neumann model fitting of our data indicates that $\beta$ of $\mathrm{G} / \mathrm{Cu}$ is $\sim 4$ times greater than the proposed universal constant and increases $\sim 7 \%$ from fresh to aged surface. Furthermore, the calculated surface energy was not sensitive to the value of $\beta$ for graphene or HOPG in this study. I.e., holding $\beta$ constant and

fitting the data did not affect calculated $\gamma_{\mathrm{s}}$, which is consistent with previous reports. ${ }^{177,} 178$ Surface energy of $\mathrm{G} / \mathrm{Cu}$ always decreased with air exposure time regardless of the model used to calculate surface energy. This decrease can be attributed to hydrocarbon contamination. 
Gomez-Herrero et al. reported aromatic hydrocarbon contamination on graphite by Kelvin probe force microscopy and suggested that the contaminants desorb near $50^{\circ} \mathrm{C} .{ }^{101}$ Additionally, Li et al. reported contamination of graphene surfaces by airborne hydrocarbons. ${ }^{77}$ Once freshly synthesized $\mathrm{G} / \mathrm{Cu}$ is exposed to ambient air, hydrocarbons adsorb onto the initially high energy surface and WCA concurrently decreases. The hydrocarbon contamination theory was substantiated with ATR-FTIR and XPS experiments showing that hydrocarbon species were initially absent and appeared after exposure to air for a period of time.$^{77}$ Moreover, recent theoretical work has suggested that graphitic surfaces are intrinsically hydrophilic. ${ }^{66,67,}{ }^{179}$ In the current work, spectroscopic ellipsometry results on fresh $\mathrm{G} / \mathrm{Cu}$ further support this conclusion. As shown in Figure 12a, the phase shift change $(\delta \Delta)$ of fresh $\mathrm{G} / \mathrm{Cu}$ sample increases with air exposure time, indicating adsorption of airborne hydrocarbon contaminants. ${ }^{180,}{ }^{181}$ Putting all this information together, surface energy is highest on the fresh (uncontaminated) $\mathrm{G} / \mathrm{Cu}$ surface and decreases as the $\mathrm{G} / \mathrm{Cu}$ surface adsorbs airborne hydrocarbons. Adsorbed contaminants "shield" the subjacent high energy surface and the sample appears hydrophobic.

Change in WCA can be related to the extent of hydrocarbon contamination as shown in Figure 12b. The ellipsometric phase shift (Figure 12a) indicates hydrocarbon contamination of fresh $\mathrm{G} / \mathrm{Cu}$ and its inverse linear relationship with $\cos (\theta)$ of the WCA provides a correlation between extent of contamination and WCA measurement. The linearity corroborates a CassieBaxter relationship, ${ }^{125,}{ }^{182}$ although the ellipsometry and WCA experiments were conducted in different laboratories, suggesting that a simple WCA measurement on $\mathrm{G} / \mathrm{Cu}$ can indicate the degree of surface contamination.

The surface energy of graphene reported by Coleman et al. is $65-90 \mathrm{~mJ} / \mathrm{m}^{2},{ }^{132}$ slightly higher than our results. However, since very different material system, experimental method and 
theoretical model was involved in their work, direct comparison is difficult. In contrast, it makes more sense to compare our results to those of Wang et al. since they also determined the surface energy by contact angle testing using the Neumann model. ${ }^{23}$ They reported $46.7 \mathrm{~mJ} / \mathrm{m}^{2}$ for the surface energy of graphene, which is significantly lower than $63.8 \mathrm{~mJ} / \mathrm{m}^{2}$ as we found for the fresh $\mathrm{G} / \mathrm{Cu}$. There are three possible reasons for the difference. First, the hydrocarbon contamination was not considered at all in the work of Wang et al. and it is not clear how that will affect the calculated surface energy in their work. Second, our graphene sample is singlelayer CVD graphene on copper while their graphene is a "thick" film synthesized by the reduction of graphene oxide. As a result, the chemistry of the two graphene samples may not be exactly the same. Third, their analysis included a fifth test liquid, formamide, which will likely influence the calculated surface energy.

\subsection{ORIGIN OF INTRINSIC POLARITY}

According to results from both Fowkes model and Owen-Wendt model, the polar component of total surface energy $\left(\gamma_{s}^{p}\right)$ of fresh $\mathrm{G} / \mathrm{Cu}$ is significant, indicating $\mathrm{G} / \mathrm{Cu}$ intrinsically has a mildly polar (hydrophilic) surface. However, since it is generally accepted that, at atomic level, graphene has non-polar $\mathrm{sp}^{2}$ structure, why is $\mathrm{G} / \mathrm{Cu}$ surface mildly polar? We suggest that the mild polarity of $\mathrm{G} / \mathrm{Cu}$ can be attributed to three factors: $\pi$-hydrogen bonding, surface defects, and partial wetting transparency.

First, a few recent theoretical studies showed that the binding energy of a water molecule on graphite is higher than previously reported, mostly in the range of $-10 \mathrm{~kJ} / \mathrm{mol}$ to -13

$\mathrm{kJ} / \mathrm{mol} .{ }^{183-185}$ Interestingly, these studies also concluded that the preferred orientation of an 
adsorbed water molecule is with the hydrogen pointing to graphene, suggesting the presence of a $\mathrm{H}^{\cdots} \pi$ interaction, ${ }^{183-185}$ also known as $\pi$-hydrogen bonding. Such interaction has been previously observed for water interacting with small conjugated molecules, such as benzene, and could be significantly enhanced by the extended $\pi$ system of graphene. ${ }^{183,}{ }^{186}$ Although further investigation is required to fully understand this effect, $\pi$-hydrogen bonding could contribute to the observed intrinsic polarity of $\mathrm{G} / \mathrm{Cu}$.

Second, the existence of defects on graphene and graphite surface could also contribute to its intrinsic polarity, as is evidenced theoretically ${ }^{187}$ and experimentally ${ }^{28,}{ }^{187}$ Also, such defect sites on graphene and graphite surfaces have been implicated as nucleation sites for adsorption of organic molecules due to their high surface energy. ${ }^{188}$ This hypothesis is supported by an increase of surface inhomogeneity indicated by increasing contact angle hysteresis upon exposure to air. ${ }^{77,169}$ It is not clear at this point if these defects are chemical or topographical in nature. However, regardless of the exact nature, these defects with high surface energy could contribute to the observed polarity of $\mathrm{G} / \mathrm{Cu}$.

Third, the copper substrate could contribute to the polarity as well. Since graphene is only one atom thick, the underlying substrate could interact with the liquid drop on top of the graphene and contribute to the observed surface energy of $\mathrm{G} / \mathrm{Cu}$. Indeed, several recent reports did show that graphene is (at least partially) transparent regarding the interaction between water and the underlying substrate. ${ }^{77,111,123}$ Shih et al. showed partial wetting transparency on graphene through molecular dynamics (MD) simulations ${ }^{111}$ and WCA results of Li et al. supported the conclusion. ${ }^{77}$ Since copper is very hydrophilic, ${ }^{185}$ it can make the G/Cu surface more polar via partial transparency effect. 
This transparency effect can be further elucidated by comparing the surface energy of $\mathrm{G} / \mathrm{Cu}$ to that of HOPG, which can be considered as infinite layers of graphene. Surface energies calculated by the Neumann, Fowkes, and Owens-Wendt models indicate that fresh HOPG exhibits surface energy of $60.2 \pm 0.9 \mathrm{~mJ} / \mathrm{m}^{2}, 55.4 \pm 3.2 \mathrm{~mJ} / \mathrm{m}^{2}$, and $51.6 \pm 0.9 \mathrm{~mJ} / \mathrm{m}^{2}$, respectively. For both the Neumann and Owens-Wendt models, the surface energy of $\mathrm{G} / \mathrm{Cu}$ is higher than HOPG. Moreover, according to Fowkes and Owens-Wendt models, the polar surface energy of $\mathrm{G} / \mathrm{Cu}$ is higher than that of HOPG. This observation is consistent with partial wetting transparency although we cannot exclude the possibility that the intrinsic defects of $\mathrm{G} / \mathrm{Cu}$ and HOPG are slightly different and could play a role. Moreover, WCA data reported by Li et al. on $\mathrm{G} / \mathrm{Cu}, \mathrm{G} / \mathrm{Ni}$, and HOPG support the partial wetting transparency effect, which suggests that the substrate may contribute to graphene polarity. ${ }^{77}$

Finally, it was recently reported that oxygen can intercalate between graphene and copper upon ambient air exposure. ${ }^{189,} 190$ Depending on the charge state of the oxygen, the intercalation could potentially increase the polarity of the $\mathrm{G} / \mathrm{Cu}$ surface and contribute to the observed time evolution of WCA. Additional studies are needed to separate the effect of hydrocarbon contamination and oxygen intercalation on the wettability of supported graphene.

\subsection{CONCLUSIONS}

This study showed that the WCA of fresh $\mathrm{G} / \mathrm{Cu}$ is $50.8^{\circ}$ and its surface energy is $62.2 \pm 3.1$ $\mathrm{mJ} / \mathrm{m}^{2}$ (Fowkes), $53.0 \pm 4.3 \mathrm{~mJ} / \mathrm{m}^{2}$ (Owens- Wendt), and $63.8 \pm 2.0 \mathrm{~mJ} / \mathrm{m}^{2}$ (Neumann). Aging in air for 1 to 2 hours consequently increases contact angle and concurrently decreases surface energy. The initially high energy graphene attracts airborne hydrocarbons, which are adsorbed on 
the graphene surface, "shield" polar surface sites, and decrease the overall surface energy. WCA of $24 \mathrm{~h}$ aged $\mathrm{G} / \mathrm{Cu}$ is $77.6^{\circ}$, and its surface energy is $45.6 \pm 3.9 \mathrm{~mJ} / \mathrm{m}^{2}$ (Fowkes), $37.5 \pm 2.3$ $\mathrm{mJ} / \mathrm{m}^{2}$ (Owens-Wendt), and $57.4 \pm 2.1 \mathrm{~mJ} / \mathrm{m}^{2}$ (Neumann). The intrinsic mild polarity of $\mathrm{G} / \mathrm{Cu}$ was explained in terms of high energy surface defects, $\pi$-hydrogen bonding, and partial wetting transparency. 


\subsection{QUANTIFYING THE DEFECT DENSITY OF GRAPHITE}

\subsection{RAMAN SPECTROSCOPY}

Pioneering work by Tuinstra and Koenig identified the characteristic peaks in the Raman spectrum of graphite. ${ }^{191}$ They observed only a single peak at $1575 \mathrm{~cm}^{-1}$ for single crystal graphite; whereas, other graphitic materials had the same peak along with an additional peak at $1355 \mathrm{~cm}^{-1}$. The former peak $\left(1575 \mathrm{~cm}^{-1}\right)$ is most prominent and attributed to the $\mathrm{E}_{2 \mathrm{~g}}$ mode of the crystalline lattice. The latter peak $\left(1355 \mathrm{~cm}^{-1}\right)$ appears upon introduction of defects to the C-C hexagonal lattice and is inversely proportional to crystallite size $\left(\mathrm{L}_{\mathrm{a}}\right){ }^{191}$ Since 1970 , this work has been greatly expanded upon by many researchers interested in better understanding the Raman signature of graphitic materials, primarily HOPG and diamond, then translating that knowledge to graphene, the 2D counterpart of graphite.

The graphitic spectrum generally has three prominent peaks of interest: D, G, and 2D. The $\mathrm{D}$ peak originates from breathing modes of the six-carbon rings, the $\mathrm{G}$ peak originates from the $\mathrm{E}_{2 \mathrm{~g}}$ phonon at the Brillouin zone center, and the $2 \mathrm{D}$ peak is the second order of the $\mathrm{D}$ peak. ${ }^{192-194}$ Additionally, other satellite peaks such as the D', D+D' $\left(2950 \mathrm{~cm}^{-1}\right)$, and 2D' (3240 $\mathrm{cm}^{-1}$ ) peaks are often observed for carbon materials. The D family of peaks originate from defects and require the presence of defects for activation (except for the 2D peak which is historically denoted as the second-order G' peak and corresponds to the overtone of the D peak); 
whereas, the $\mathrm{G}$ peak originates from phonon activity and is observed in all graphitic materials. ${ }^{195,}$ ${ }^{196}$ Graphene and graphite provide a unique opportunity for elucidating the Raman response of $\mathrm{sp}^{2}$-hybridized carbon because experiments can be performed on the material from the bulk phase down to the atomic phase.

Ferrari and Robertson provided an extensive investigation into the Raman spectra of carbon materials differentiating the signatures of $\mathrm{sp}^{2}$ - and $\mathrm{sp}^{3}$-hybridized carbon. Similar to the attributions by Tuinstra and Koenig, the $\mathrm{D}$ was attributed to breathing of the $\mathrm{A}_{1 \mathrm{~g}}$ symmetry phonons, i.e., breathing of the aromatic carbon rings. The characteristic $\mathrm{G}$ peak was attributed to relative motion of $\mathrm{sp}^{2}$ carbon atoms. ${ }^{192}$ The attribution of the $\mathrm{D}$ peak to signify defects or disorder was further solidified through studies by Compagnini et al. where they investigated differences in the basal and edge planes of HOPG, ${ }^{197}$ Das et al. by comparing HOPG with single and multi layer graphene, ${ }^{193}$ and several other important studies which focused on the surfaces of HOPG and graphene. ${ }^{194,198-202}$

Defects are broadly defined as anything that disrupts the infinite lattice structure of a crystalline material. $^{203}$ Point defects include vacancies, substitutional impurity atoms, selfinterstitial atoms, and interstitial impurity atoms as shown in Figure 13. See Chapter 2 in Structure-Property Relations In Nonferrous Metals for a detailed discussion. ${ }^{204}$ Defects can be introduced intentionally to the graphite and graphene surface by irradiating with ions of a particular energy. Collision events between the incident ions and carbon surface will damage the $\mathrm{sp}^{2}$ carbon lattice, effectively creating point defects with $\mathrm{sp}^{3}$-hybridized C-C bonding, vacancylike defects, and step edges. The number and size of defects can be precisely tuned by controlling the ion $\left({ }^{112} \mathrm{Sn},{ }^{209} \mathrm{Bi}, \mathrm{Ar}, \mathrm{Mn}\right.$, etc. $)$ along with its energy. ${ }^{194,198,199,205}$ This level of control has allowed researchers to investigate the precise Raman response to a particular level of 
defects, thus Raman provides the capability to quantitatively determine the defect density of graphitic surfaces. This is because intensity of the $G$ peak $\left(I_{G}\right)$ is independent of disorder while intensity of the D peak $\left(I_{D}\right)$ is directly proportional to disorder up to amporphization where the $\mathrm{sp}^{2}$ aromatic ring is destroyed. ${ }^{192,194}$ Natural graphite and HOPG inherently have point defects on their surface while maintaining the $\mathrm{sp}^{2} \mathrm{C}-\mathrm{C}$ structure; therefore, the focus of this study is to elucidate the intrinsic defect density of graphitic samples using Raman spectroscopy.

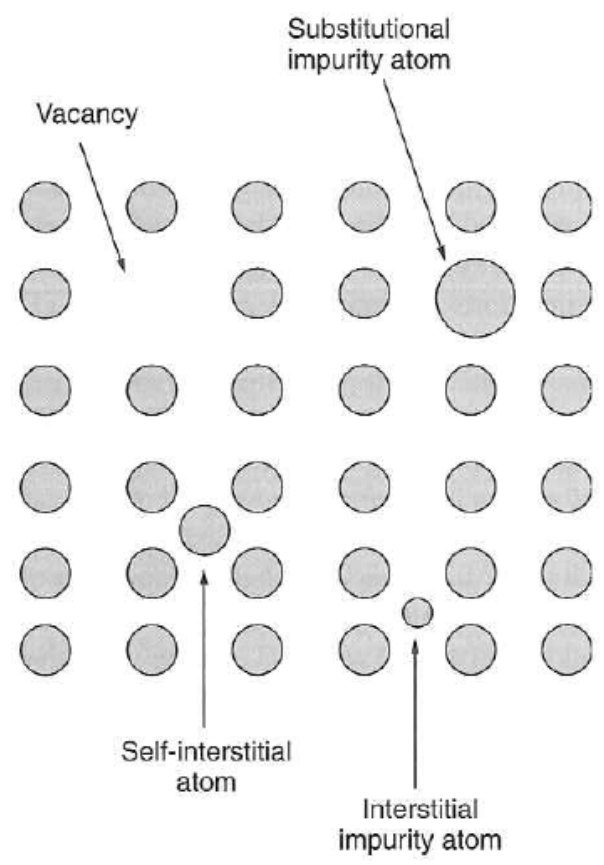

Figure 13. Point defects that occur in a crystal lattice. From Russell and Lee, StructureProperty Relations In Nonferrous Metals. Copyright (C) 2005 by John Wiley Sons, Inc. Reprinted by permission of John Wiley \& Sons, Inc. ${ }^{204}$ 


\subsubsection{Raman data acquisition and analysis}

Raman spectra of ZYA and PG were collected using a custom-built spectrometer with a $532 \mathrm{~nm}$ solid-state laser and 40x (NA: 0.60) optical objective. Additional details can be found in Reference 77. The sample was cleaved either with tape (ZYA) or razor (PG) before data acquisition for 5 min. Figure 14 shows the Raman spectra of ZYA and PG along with the corresponding peak attributions. The G peak is most salient in the spectra of both materials, as expected for graphite. Absence of the D' peak for both samples indicates that the samples are of relatively low defect density because this peak would appear above a certain level of disorder. ${ }^{205}$ The D peak is easily seen for PG but is absent for ZYA. This indicates that a significantly greater level of Raman detectable defects (i.e., point defects, step edges, etc.) are present on PG.

The 2D peak around $2700 \mathrm{~cm}^{-1}$ is observed for both samples, but with different line shapes. The characteristic $2 \mathrm{D}$ peak for HOPG is observed in the spectra of ZYA with a sharp peak and a shoulder towards lower wavenumber. Contrarily, the $2 \mathrm{D}$ peak for PG is a single sharp peak that is redshifted $13.5 \mathrm{~cm}^{-1}$ compared to ZYA. The 2D peak lineshape classifies ZYA and PG as polycrystalline graphite. ${ }^{206}$ Lespade et al. showed that a single 2D peak occurs for turbostratic graphite (i.e., graphite without $\mathrm{AB}$ interlayer stacking). ${ }^{207}$ This result was confirmed through work by Ferrari et al. which demonstrated that the 2D peak lineshape is a function of the number of graphene layers approaching the lineshape of graphite at 5 layers. ${ }^{168}$ Further details of the 2D peak behaviour for HOPG and turbostratic graphite can be found in Raman Spectroscopy in Graphene Related Systems by Jorio et al. ${ }^{196}$ Single layer graphene has no interlayer coupling with adjacent graphene layers resulting in a single 2D peak; whereas, interlayer coupling in multilayer graphene resolves two $2 \mathrm{D}$ peaks $\left(\mathrm{D}_{1}\right.$ and $\left.\mathrm{D}_{2}\right){ }^{208}$ Thus, the $2 \mathrm{D}$ peak lineshape of ZYA qualifies this sample as high quality graphite with $\mathrm{ABAB}$ graphene stacking. The single 2D peak 
of $\mathrm{PG}$, along with its peak position nearer $\mathrm{D}_{2}$, indicates that $\mathrm{PG}$ is turbostratic graphite with less (or no) interlayer coupling. This finding is corroborated by x-ray diffraction (Appendix B on page 143) which shows that interlayer spacing of PG is $3.43 \AA$ which is larger than the typical $3.35 \AA$ interlayer spacing of HOPG. ZYA has an interlayer spacing of $3.36 \AA$.
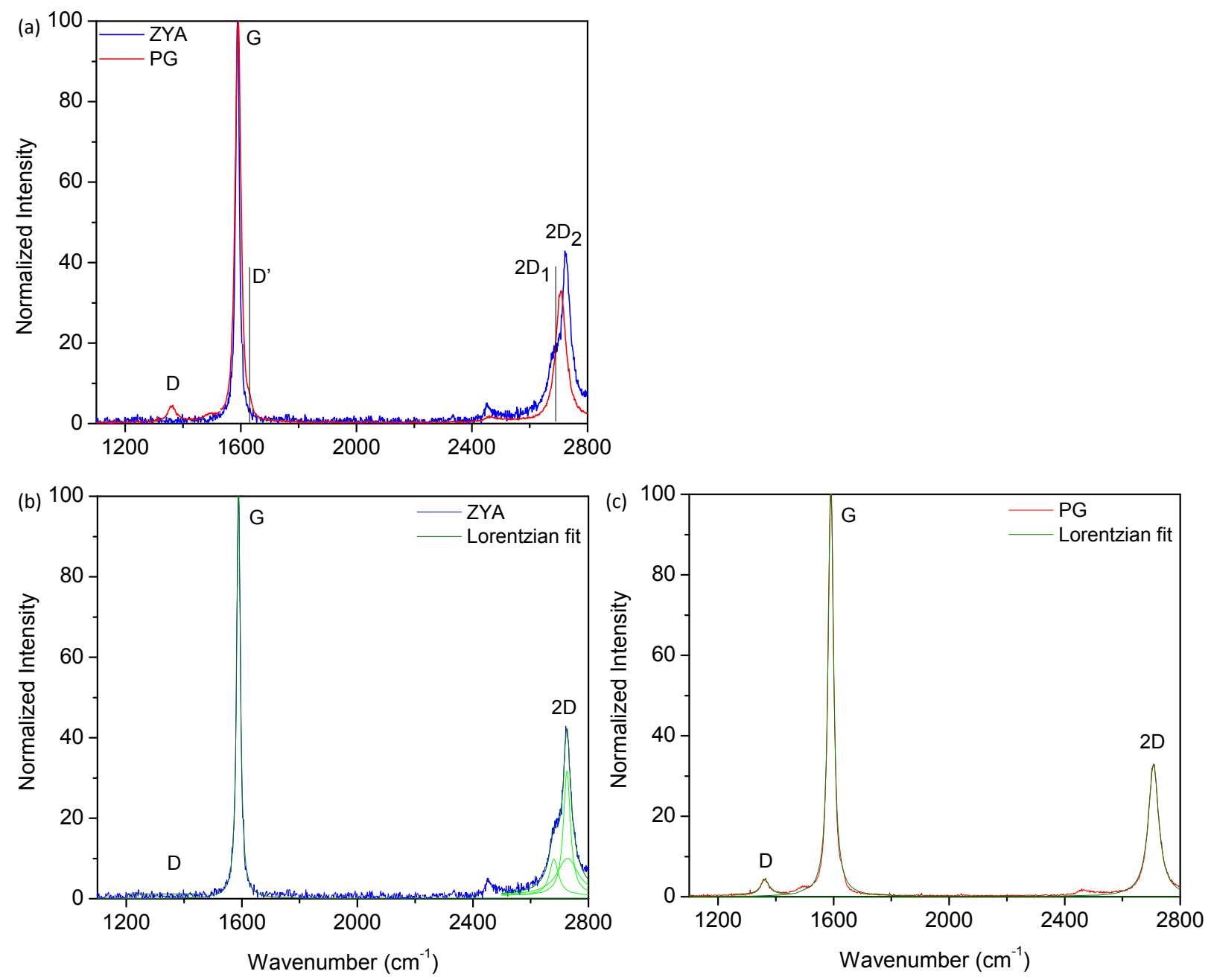

Figure 14. Raman spectra of graphite samples. (a) Normalized spectra of ZYA of PG with peak attributions. (b) ZYA spectrum with the D and G peaks each fit with a single Lorentzian and the 2D peak fit with three Lorentzian curves. (c) PG spectrum with the $\mathrm{D}, \mathrm{G}$, and 2D peaks each fit with a single Lorentzian. 
Surface enhanced Raman scattering (SERS) is a Raman response which is a consequence of sample surface roughness among other factors. ${ }^{209,} 210$ In this study, SERS causes the raw intensity of the PG spectrum to be significantly greater than the ZYA spectrum, manifesting as greater peak intensity for PG because of its rougher surface. Normalizing the data results in the spectrum of PG appearing smoother than the spectrum of ZYA although Raman data for both samples were taken on the same experimental setup using the same parameters. The less noise apparent in the PG spectrum is due to SERS/normalizing and does not influence conclusions made from the spectra.

\subsubsection{Surface sensitivity}

Raman spectroscopy is not a surface sensitive technique and probes into the bulk of the sample, thus defects detected by Raman originate throughout the sample and not just at its surface. WCA, on the other hand, is a very surface sensitive technique and probes only the uppermost layers of the sample. ${ }^{211}$ Therefore, in order to quantify only surface defects through Raman spectroscopy and correlate to wettability, it is necessary to determine the contribution of surface defects compared to the total amount of Raman detectable defects.

The Beer-Lambert equation can be used to calculate penetration depth of the Raman laser into graphite:

$$
\frac{\mathrm{I}}{\mathrm{I}_{\mathrm{o}}}=\mathrm{e}^{-\alpha \mathrm{z}}=\frac{1}{\mathrm{e}} \quad \text { Equation } 24
$$


where $\alpha=4 \pi \mathrm{k} / \lambda, \mathrm{k}$ is the extinction coefficient of graphite $(\mathrm{k}=1.3)$, and $\lambda$ is the laser wavelength $(\lambda=532 \mathrm{~nm})$. The penetration depth (z) is calculated to be $32.6 \mathrm{~nm}$ which is equivalent to $c a .97$ graphene layers. This means that Raman detectable defects originate from the uppermost 97 graphene layers. To estimate the $I_{D} / I_{G}$ ratio of the uppermost layer of graphene, the following assumptions are made: ${ }^{76}$

1. Only the top layer of graphene contributes to the $\mathrm{D}$ peak.

2. Each graphene layer adsorbs $2.3 \%$ of incoming laser light as well as the Raman signal from the underlying graphene layers.

3. The Raman emission from each graphene layer is proportional to the laser intensity it experiences.

4. Exfoliation does not cause additional Raman detectable defects.

With these assumptions, contribution of each graphene layer to the observed $G$ peak intensity will decrease by $(1-0.023)^{2}$ for each increase of layer depth; contribution from the top layer of graphene accounts for $4.5 \%$ of the observed G peak intensity. ${ }^{76}$ This is reflected in Table 7 where $\mathrm{I}_{\mathrm{G}}$ is $4.5 \%$ of the actual experimental peak height to correct for surface sensitivity.

We assume that only the top layer of graphene contributes to the D peak because defects are concentrated on the sample surface and subjacent graphene layers are pristine and defect free. While this assumption may not perfectly describe the sample and subjacent graphene layers may have some defects, defects will be substantially restricted to the uppermost graphene layer. ${ }^{76,192,}$ 212 


\subsubsection{Raman spectra peak fitting}

Ferrari and Robertson note the ambiguity of using peak heights or peak areas to calculate $\mathrm{I}_{\mathrm{D}} / \mathrm{I}_{\mathrm{G}}{ }^{192}$ They state that the difference between using peak heights or areas is not significant for disordered graphite; the difference becomes salient for amorphous carbon where peak height correlates to aromatic ring distortion and peak width describes the order and dimension of aromatic clusters. Moreover, peak height is typically used when Raman peaks are fit using the Breit-Wigner-Fano (BWF) and Lorentzian functions while peak area is used for Gaussian fits. ${ }^{192}$ Cançado et al. also noted this distinction and used peak heights. ${ }^{194}$ Therefore, peak height will be used for subsequent analysis in accordance with this reasoning.

The BWF and Lorentzian functions are commonly used to fit Raman spectra of carbon materials. Typically the Lorentzian is used for the D and 2D peaks while BWF is used for the G peak due to its asymmetry in some materials. ${ }^{192}$ The G peak for ZYA and PG is very symmetric thus all three peaks (D, G, and 2D) were fit using the Lorentzian function. A single Lorentzian was used for all peaks except for the 2D peak of ZYA which required deconvolution into three Lorentzian peaks. ${ }^{168,192,208}$ The D peak for ZYA was not resolved indicating very little to no Raman detectable defects. Nonetheless, the peak center $\left(\mathrm{x}_{\mathrm{c}}=1362.0 \mathrm{~cm}^{-1}\right)$ was held constant and the peak was fit to allow calculation of the defect density.

Fitting results shown in Table 7 and Table 8 illustrate several differences between ZYA and PG. FWHM of the G peak is $15 \mathrm{~cm}^{-1}$ for ZYA, corresponding to the literature value for HOPG, and $22 \mathrm{~cm}^{-1}$ for PG. ${ }^{192,196,213,214}$ Since the G peak width is related to charge doping (e.g., intercalates, defects, etc.) ${ }^{215-217} \mathrm{PG}$ is qualified as having more defects than ZYA based on (a) presence of the D peak, (b) wider G peak, and (c) redshifted 2D peak resolved as a single 
peak. ${ }^{191,206,214}$ Moreover, Raman and XRD (Appendix B on page 143) spectra indicate that PG is turbostratic which is expected to have more defects than HOPG. The intrinsic disorder in ZYA and PG is well within the first stage of disorder classification proposed by Ferrari et al. considering (a) the expected graphitic nature of the samples, (b) FWHM of the G peak, (c) position of the G peak, and (d) sharp well defined D, G, and 2D peaks. ${ }^{192}$ This validates the use of Equation 25, Equation 26, and Equation 27 to calculate defect density since these equations were derived from samples within the first stage of disorder (Section 4.1.4). ${ }^{194}$

Equation 27 provides a quantitative method for determining the defects on the uppermost surface of graphite (1 graphene layer). The top layer of graphene accounts for $4.5 \%$ of the observed $G$ peak intensity (Section 4.1.2), thus $\mathrm{I}_{\mathrm{G}}$ was multiplied by 0.045 to obtain the contribution from the top layer of graphene and this is reflected in Table 7.

Table 7. Raman spectra fitting results of ZYA and PG. Data fit using Lorentzian functions. Intensity (I) is determined from the peak height which is an extrinsic parameter of the Lorentzian function, thus there is no associated error. $\mathrm{I}_{2 \mathrm{D}}$ and $\mathrm{FWHM}_{2 \mathrm{D}}$ for ZYA are taken from the cumulative fit of the three sub-peaks. The fitting error of $\mathrm{FWHM}_{\mathrm{D}}$ for ZYA is unrealistically large because the peak was forced to fit yet no peak was conspicuously resolved.

\begin{tabular}{|c|c|c|c|c|c|c|c|c|}
\hline & $\mathrm{I}_{\mathrm{D}}$ & $\mathrm{I}_{\mathrm{G}}$ & $\mathrm{I}_{2 \mathrm{D}}$ & $\mathrm{I}_{\mathrm{D}} / \mathrm{I}_{\mathrm{G}}$ & $\mathrm{I}_{\mathrm{G}} / \mathrm{I}_{2 \mathrm{D}}$ & $\mathrm{FWHM}_{\mathrm{D}}$ & $\mathrm{FWHM}_{\mathrm{G}}$ & $\mathrm{FWHM}_{2 \mathrm{D}}$ \\
\hline ZYA & 0.05 & 4.59 & 42.31 & 0.01090 & 0.11 & $\begin{array}{c}0.42 \\
( \pm 889.00)\end{array}$ & $\begin{array}{c}15.34 \\
( \pm 0.79)\end{array}$ & $\begin{array}{c}40.02 \\
( \pm 5.58)\end{array}$ \\
\hline $\mathrm{PG}$ & 3.78 & 4.64 & 32.84 & 0.81506 & 0.14 & $\begin{array}{c}34.17 \\
( \pm 1.64)\end{array}$ & $\begin{array}{c}22.34 \\
( \pm 0.69)\end{array}$ & $\begin{array}{c}44.04 \\
( \pm 5.75)\end{array}$ \\
\hline
\end{tabular}


Table 8. Raman spectra peak centers of ZYA and PG. $\mathrm{x}_{\mathrm{c}}$ of the D peak for ZYA was held constant to allow fit to converge.

\begin{tabular}{|c|c|c|c|c|c|}
\hline & $\mathrm{D}$ & $\mathrm{G}$ & $2 \mathrm{D}_{1}$ & $2 \mathrm{D}_{2}$ & $2 \mathrm{D}_{3}$ \\
\hline ZYA & $1362.0 \mathrm{~cm}^{-1}$ & $\begin{array}{c}1588.5 \\
( \pm 0.3) \mathrm{cm}^{-1}\end{array}$ & $\begin{array}{c}2680.3 \\
( \pm 1.3) \mathrm{cm}^{-1}\end{array}$ & $\begin{array}{c}2725.4 \\
( \pm 0.3) \mathrm{cm}^{-1}\end{array}$ & $\begin{array}{c}2727.7 \\
( \pm 6.7) \mathrm{cm}^{-1}\end{array}$ \\
\hline PG & $\begin{array}{c}1362.0 \\
( \pm 0.4) \mathrm{cm}^{-1}\end{array}$ & $\begin{array}{c}1590.4 \\
( \pm 0.2) \mathrm{cm}^{-1}\end{array}$ & -- & $\begin{array}{c}2707.7 \\
( \pm 2.0) \mathrm{cm}^{-1}\end{array}$ & -- \\
\hline
\end{tabular}

\subsubsection{Calculating defect density}

There are several different types of defects that occur on graphite: primarily step edges, dislocations, cracks, vacancies, and point defects. ${ }^{192-194}$ Raman spectroscopy is very good at detecting point defects which are the smallest type of defect and unable to be detected by traditional AFM; ${ }^{194}$ whereas, well-defined zigzag edge defects are not detectable by Raman. ${ }^{200-}$ 202 The ability to detect intrinsic point defects on graphite and characterize surface quality both quantitatively and qualitatively is invaluable for comparing samples of varying quality, especially for samples with relatively low defect density that have not been intentionally damaged.

Cançado et al. showed that $\mathrm{I}_{\mathrm{D}} \propto\left(\mathrm{L}_{\mathrm{L}} / \mathrm{L}_{\mathrm{d}}\right)^{2}$ where $\mathrm{L}_{\mathrm{L}}$ is the Raman laser spot size and $\mathrm{L}_{\mathrm{d}}$ is the average distance between defects. ${ }^{194}$ This qualitatively makes sense because $\mathrm{I}_{\mathrm{D}}$ increases with defect density (i.e., less distance between defects means greater defect density and larger $\mathrm{I}_{\mathrm{D}}$ ). Additionally, $\mathrm{I}_{\mathrm{G}}$ is independent of defect density: $\mathrm{I}_{\mathrm{G}} \propto \mathrm{L}_{\mathrm{L}}{ }^{2}$. ${ }^{194}$ Combining these equations yields $\mathrm{I}_{\mathrm{D}} / \mathrm{I}_{\mathrm{G}} \propto 1 / \mathrm{L}_{\mathrm{d}}{ }^{2} \cdot{ }^{192,194,199}$ The distance between defects $\left(\mathrm{L}_{\mathrm{d}}\right)$ can be calculated from $\mathrm{I}_{\mathrm{D}} / \mathrm{I}_{\mathrm{G}}$ by Equation 25 and defect density can be calculated from Equation 26 where $\mathrm{E}_{\mathrm{L}}$ is the laser energy $\left(\lambda=532 \mathrm{~nm} ; \mathrm{E}_{\mathrm{L}}=2.33 \mathrm{eV}\right):{ }^{194}$ 


$$
\begin{array}{cc}
\mathrm{L}_{\mathrm{d}}^{2}\left(\mathrm{~nm}^{2}\right)=\frac{(4300 \pm 1300)}{\mathrm{E}_{\mathrm{L}}^{4}}\left(\frac{\mathrm{I}_{\mathrm{D}}}{\mathrm{I}_{\mathrm{G}}}\right)^{-1} & \text { Equation } 25 \\
\mathrm{n}_{\mathrm{d}}\left(\text { defects } / \mathrm{cm}^{2}\right)=\frac{10^{14}}{\pi \mathrm{L}_{\mathrm{d}}^{2}} & \text { Equation } 26
\end{array}
$$

Therefore, combining Equation 25 and Equation 26 allows for the defect density to be calculated:

$$
\mathrm{n}_{\mathrm{d}}\left(\text { defects } / \mathrm{cm}^{2}\right)=(7.4 \mathrm{e} 9 \pm 2.5 \mathrm{e} 9) \mathrm{E}_{\mathrm{L}}^{4}\left(\frac{\mathrm{I}_{\mathrm{D}}}{\mathrm{I}_{\mathrm{G}}}\right) \quad \text { Equation } 27
$$

The previous equations (Equation 25, Equation 26, and Equation 27) were empirically determined for single layer graphene on $\mathrm{SiO}_{2}$, thus they are relevant only for the uppermost graphite surface (see Section 4.1.2 on page 56 for calculations pertaining to Raman sensitivity), which is also the area of interest in WCA analysis. ${ }^{194,} 199$ These equations have also been used to calculate defect density on HOPG. ${ }^{198}$

An important consideration for graphite samples is the size of crystalline domains (i.e., crystallite size). ${ }^{214}$ Knight and White showed that the crystallite size $\left(L_{a}\right)$ is inversely related to the ratio $\mathrm{I}_{\mathrm{D}} / \mathrm{I}_{\mathrm{G}}\left(\mathrm{L}_{\mathrm{a}} \propto 1 /\left(\mathrm{I}_{\mathrm{D}} / \mathrm{I}_{\mathrm{G}}\right)\right)$ for $2.5<\mathrm{L}_{\mathrm{a}}<300 \mathrm{~nm} .{ }^{191,192,206}$ The average crystallite size for ZYA and PG are unknown but $L_{a}$ is proportional to the distance between defects $\left(L_{d}\right)$ so one can roughly estimate that the actual crystalline domain is roughly equivalent to the distance between defects; therefore, $\mathrm{L}_{\mathrm{a}} \propto \mathrm{L}_{\mathrm{d}} \propto 1 /\left(\mathrm{I}_{\mathrm{D}} / \mathrm{I}_{\mathrm{G}}\right)$.

Calculation results using Raman spectra are shown in Table 9. Distance between defects is substantially larger for ZYA than PG, roughly $116 \mathrm{~nm}$ and $13 \mathrm{~nm}$, respectively. Defect 
density of ZYA and PG is ca. 24 and 1778 defects $/ \mu \mathrm{m}^{2}$. Assuming that the domains are square, the domain area for ZYA and PG is calculated to be $13,380 \mathrm{~nm}^{2}$ and $179 \mathrm{~nm}^{2}$, respectively. The domains are $c a$. 75x larger on ZYA. Likewise, ZYA has substantially fewer defects per carbon atom than PG. In fact, ZYA has about an order of magnitude less defects than CVD synthesized graphene on copper (see Table S1 of Reference 77): the defect density of G/Cu and ZYA is $c a$. $4.5 \times 10^{10} \mathrm{defects} / \mathrm{cm}^{2}$ and $0.24 \times 10^{10} \mathrm{defects} / \mathrm{cm}^{2}$, respectively. ${ }^{77}$ For comparison, SPI-2 has $5.1 \times 10^{10}$ defects $/ \mathrm{cm}^{2}$ and less than $13 \times 10^{-6}$ defects/carbon atom, which is a greater defect density than ZYA. ${ }^{76}$

Interestingly, tip-enhanced Raman spectroscopy (TERS) can be used to probe individual point defects on graphitic surfaces. This technique can be used to directly elucidate distance between defects $\left(\mathrm{L}_{\mathrm{d}}\right){ }^{218}$ Raman spectroscopy has also been shown to be useful in analyzing defects on $\mathrm{MoS}_{2}{ }^{219}$ Using Raman, Cho et al. showed that initial defects on graphene field effect transistors (FETs) substantially influence its electronic properties and decreasing surface defects is critical for improving device performance. ${ }^{220}$ Further information on Raman characterization of graphene defects can be found in a 2015 review by Beams, Cançado, and Novotny. ${ }^{221}$ Scanning tunneling microscopy (STM) may be an alternative technique for imaging point defects in graphite in order to verify Raman data. ${ }^{222-224}$

Using the defect density in Table 9, percentage of defects on the graphite surface can be calculated assuming that defects are circular with radius of $73 \mathrm{pm}$. These calculations are discussed in detail in Section 5.3 .2 (page 104) pertaining to the Cassie-Baxter model. Interestingly, the defect density of both graphite samples is extremely low: ZYA and PG have defect density of $0.00004 \%$ and $0.00298 \%$, respectively $\left(r_{d}=73 \mathrm{pm}\right)$. Considering that $P G$ is 
low quality graphite and ZYA is high quality graphite, it is reasonable to expect PG to have more defects.

Table 9. Defect calculations for graphite samples. $L_{d}$ is the distance between defects and $n_{d}$ is the defect density. Domain size is calculated assuming the defect free area is a square. The number density of carbon atoms is $3.85 \times 10^{15} / \mathrm{cm}^{2}$.

\begin{tabular}{|c|c|c|c|c|}
\hline & $\begin{array}{c}\mathrm{L}_{\mathrm{d}} \\
(\mathrm{nm})\end{array}$ & $\begin{array}{c}\text { Defect Density } \\
\mathrm{n}_{\mathrm{d}}\left(\mathrm{defects} / \mu^{2}\right)\end{array}$ & $\begin{array}{c}\text { Domain area: } \mathrm{L}_{\mathrm{a}} \\
\left(\mathrm{nm}^{2}\right)\end{array}$ & $\begin{array}{c}\text { Defects per } \\
\text { carbon atom } \\
\left(\mathrm{x} 10^{-6}\right)\end{array}$ \\
\hline ZYA & $\begin{array}{c}116 \\
( \pm 16)\end{array}$ & $\begin{array}{c}24 \\
( \pm 16)\end{array}$ & $\begin{array}{c}13,380 \\
( \pm 3778)\end{array}$ & $\begin{array}{c}0.6 \\
( \pm 0.4)\end{array}$ \\
\hline $\mathrm{PG}$ & $\begin{array}{c}13 \\
( \pm 2)\end{array}$ & $\begin{array}{c}1778 \\
( \pm 1187)\end{array}$ & $\begin{array}{c}179 \\
( \pm 51)\end{array}$ & $\begin{array}{c}46.2 \\
( \pm 30.8)\end{array}$ \\
\hline
\end{tabular}

Diameter of the Raman laser spot can be calculated by Equation 28:

$$
\text { laser spot diameter }=1.22 \frac{\lambda}{\mathrm{NA}} \quad \text { Equation } 28
$$

where $\lambda$ is laser wavelength $(532 \mathrm{~nm})$ and NA is the numerical aperture of the optical objective $(0.60){ }^{225}$ Therefore, the laser spot diameter is $1.08 \mu \mathrm{m}$ and the spot area is $0.92 \mu \mathrm{m}^{2}$, assuming a circular spot. Knowing the laser spot area and the defect density, the number of defects detected by the Raman experiment can be calculated: 


$$
\begin{array}{ll}
\frac{0.92 \mu \mathrm{m}^{2} \text { laser area }}{13,380 \mathrm{~nm}^{2} \text { defect free area }} \mid \frac{1000^{2} \mathrm{~nm}^{2}}{1^{2} \mu \mathrm{m}^{2}}=69 \text { defects on ZYA } & \text { Equation } 29 \\
\frac{0.92 \mu \mathrm{m}^{2} \text { laser area }}{179 \mathrm{~nm}^{2} \text { defect free area }} \mid \frac{1000^{2} \mathrm{~nm}^{2}}{1^{2} \mu \mathrm{m}^{2}}=5,140 \text { defects on PG } & \text { Equation } 30
\end{array}
$$

The Raman laser detects $c a .69$ and 5,140 defects on ZYA and PG, respectively. These values are lower limit estimates because Equation 28 is valid when the aperture and laser are equal in diameter. Our Raman setup uses a laser diameter smaller than the back aperture diameter, thus the actual laser spot size incident on the sample is larger than that calculated by Equation 28. Nonetheless, the number of defects are proportional to laser spot size and PG has significantly more surface defects than ZYA.

AFM images (Figure 15 on page 70) of ZYA show that the surface is primarily smooth basal plane with few line defects (i.e., edges or ridges between basal planes; essentially a graphene step edge). The lack of D peak for ZYA indicates that these defects are either not resolvable by Raman or the Raman laser does not contact enough defects because ZYA is primarily pristine basal plane. In fact, the latter is most likely because the Raman laser detected ca. 69 defects on ZYA. Contrarily, AFM images of PG show a bubbly surface without any well defined step edges; however, defects are clearly observed in the spectrum and the laser detected ca. 5,140 defects on PG.

Pollard et al. presented an alternative method of relating $\mathrm{I}_{\mathrm{D}} / \mathrm{I}_{\mathrm{G}}$ to the defect density:

$$
\frac{I_{D}}{I_{G}}=C_{A} \frac{\left(r_{A}^{2}-r_{S}^{2}\right)}{\left(r_{A}^{2}-2 r_{S}^{2}\right)}\left[e^{-\pi r_{S}^{2} / L_{D}^{2}}-e^{-\pi\left(r_{A}^{2}-r_{S}^{2}\right) / L_{D}^{2}}\right] \quad \text { Equation } 31
$$


where $\mathrm{C}_{\mathrm{A}} \approx 160 / \mathrm{E}_{\mathrm{L}}{ }^{4}, \mathrm{r}_{\mathrm{s}}$ is the lateral radius of the vacancy defect, $\mathrm{L}_{\mathrm{o}}$ is the relaxation length which equals $2.4 \pm 0.6 \mathrm{~nm}$, and $\mathrm{r}_{\mathrm{A}}=\mathrm{r}_{\mathrm{s}}+\mathrm{L}_{\mathrm{o}}{ }^{205}$ The vacancy defect radius can be determined by scanning tunneling microscopy (STM) and is $0.6 \pm 0.1 \mathrm{~nm}$ for $\mathrm{Mn}^{+}$ion bombardment. While ZYA and PG are expected to have little to no vacancy defects, Equation 31 can still be used to roughly estimate $L_{D}$ and compare the value to that obtained by Equation 25 . When $I_{D} / I_{G}$ $=0.01090$ (Table 7), $\mathrm{L}_{\mathrm{D}}$ is calculated to be $116.2 \mathrm{~nm}$, which perfectly matches the value calculated using Equation 25. Decreasing the vacancy defect radius $\left(r_{s}\right)$ to $0.1 \mathrm{~nm}$, which is reasonable for a sample that has undergone no ion bombardment, results in $L_{D}=98.7 \mathrm{~nm}$. Qualitatively, this still provides a reasonable value for ZYA; therefore, the defect density calculated by Equation 27 matches well with alternative empirical equations currently available in literature.

The D and D' peaks are defect activated and their ratio can be used to elucidate the nature of defects. Eckmann et al. showed that the $\mathrm{I}_{\mathrm{D}} / \mathrm{I}_{\mathrm{D}}$, is $\sim 13$ for $\mathrm{sp}^{3}$ defects, $\sim 7$ for vacancy-like defects, and $\sim 3.5$ for graphitic boundaries. ${ }^{203}$ Using this relationship and Figure 14, both ZYA and PG have no D' peak resolved, thus a very large $\mathrm{I}_{\mathrm{D}} / \mathrm{I}_{\mathrm{D}}$ ' can be estimated, indicating that the defects on both graphitic samples are primarily point defects. Moreover, lack of a D' peak indicates that both samples have a relatively low intrinsic defect density since density of these Raman detectable defects are too low to be resolved. ${ }^{195}$ Analysis of Raman spectra unequivocally show that PG has many more defects and substantially smaller crystallite domains than ZYA. This further qualifies Raman spectroscopy as a quick, easy, and nondestructive method for quantitatively and qualitatively determining defect density in graphite.

Mallet-Ladeira et al. recently proposed an alternative method which is more accurate when $\mathrm{La} \leq 10 \mathrm{~nm}$. Using coke and pyrocarbons, they showed that the relationships derived by 
Tuinstra and Koenig fail at small crystallite sizes $(\leq 10 \mathrm{~nm})$. Furthermore, they report that the relationship between $\mathrm{E}_{\mathrm{L}}$ and Raman signal becomes increasingly uncertain as crystallite size increases above $10 \mathrm{~nm}$ regardless of the model used. ${ }^{226}$ This caveat is important to note and may impact calculations; however, absent an improved model for large crystalline graphite, the currently available relationships between laser excitation energy and crystallite size were still used.

\subsubsection{Number of defects in WCA experiments}

The water drop forms a perfect hemisphere on the sample surface when its WCA is $90^{\circ}$ and deviation increases as WCA either increases or decreases, yet assuming a hemisphere shape remains a valid approximation for estimating the area of water contacting the sample. The volume of a hemisphere is calculated using $V=\frac{2}{3} \pi r^{3}$ and solving for radius (r) gives: $r=\sqrt[3]{3 \mathrm{~V} / 2 \pi}$. The volume of water used in experiments was $2 \mu \mathrm{L}$ which gives a drop radius of $0.99 \mathrm{~mm}$. Physically measuring the actual water drop diameter yields a value near $2 \mathrm{~mm}$ which is in agreement with the calculation.

The area of the hemisphere base is analogous to the area of a circle, thus the area of the water drop contacting the sample is calculated using $\mathrm{A}=\pi \mathrm{r}^{2}$ which gives an area of $3.05 \mathrm{~mm}^{2}$. Physically, this means that the area of water contacting the graphite surface is $3.05 \mathrm{~mm}^{2}$. Using the defect density in Table 9, the calculated number of defects contacted by each water drop in the WCA experiments was 72.5 million and 5.4 billion for ZYA and PG, respectively: 


$$
\text { Defects on aged ZYA }=23.8 \frac{\text { defects }}{\mu \mathrm{m}^{2}} \mid \frac{1000^{2} \mu \mathrm{m}^{2}}{1^{2} \mathrm{~mm}^{2}} \times 3.05 \mathrm{~mm}^{2} \text { drop area }=7.25 \mathrm{e} 7 \text { defects }
$$

Equation 32

The water drop is a hemisphere only when its contact angle is $90^{\circ}$. The fresh and aged WCA for ZYA is $65.6^{\circ}$ and $91.0^{\circ}$, respectively; whereas the fresh and aged WCA for PG is $47.4^{\circ}$ and $91.0^{\circ}$, respectively (see Table 11 on page 78 ). In this case, the water drop can be modeled as a spherical cap where Equation 33 is used when WCA $<60^{\circ}$ and Equation 34 is used when WCA $\geq 60^{\circ} \cdot{ }^{227}$ Equation 33 was used for the fresh PG surface and Equation 34 was used for the others; the water drop was assumed to be axially symmetric. ${ }^{228}$ Table 10 summarizes the calculated number of defects that the water drop contacts on the graphitic surface. Unsurprisingly, the water contacts more surface defects when it spreads (i.e., more hydrophilic fresh surface) compared to the contaminated hydrophobic surface. Based solely on drop radius, water placed on fresh ZYA and PG contacts $c a .50 \%$ and $150 \%$ more defects than water placed on aged ZYA and PG, respectively.

$$
\begin{array}{cc}
r_{\text {hydrophilic }}=\left(\frac{6 \mathrm{~V} \sin 3 \theta}{\pi(1-\cos \theta)\left(3 \sin ^{2} \theta+(1-\cos \theta)^{2}\right)}\right)^{1 / 3} & \text { Equation } 33 \\
r_{\text {hydrophobic }}=\left(\frac{-3 V \sin ^{3} \beta}{\pi\left((1-\cos \beta)^{2}(2+\cos \beta)-4\right)}\right)^{1 / 3} \text { where } \beta=180^{\circ}-\theta & \text { Equation 34 }
\end{array}
$$


Table 10. Number of surface defects contacted by water drop. A $2 \mu \mathrm{L}$ water drop was used in experiments and calculations.

\begin{tabular}{|c|c|c|c|c|c|c|c|c|}
\hline & \multicolumn{4}{|c|}{ Fresh surface } & \multicolumn{4}{c|}{ Aged surface } \\
\cline { 2 - 9 } & WCA & $\mathrm{r}(\mathrm{mm})$ & Defects & $\begin{array}{c}\text { C atoms } \\
\left(\mathrm{x} 10^{14}\right)\end{array}$ & WCA & $\begin{array}{c}\mathrm{r} \\
(\mathrm{mm})\end{array}$ & Defects & $\begin{array}{c}\mathrm{C} \text { atoms } \\
\left(\mathrm{x} 10^{14}\right)\end{array}$ \\
\hline ZYA & $65.4^{\circ}$ & 1.204 & $\begin{array}{c}108 \text { million } \\
( \pm 72 \text { million })\end{array}$ & 1.8 & $91.0^{\circ}$ & 0.976 & $\begin{array}{c}71 \text { million } \\
( \pm 48 \text { million })\end{array}$ & 1.2 \\
\hline PG & $48.0^{\circ}$ & 1.566 & $\begin{array}{c}13.7 \text { billion } \\
( \pm 9.2 \text { billion })\end{array}$ & 3.0 & $91.0^{\circ}$ & 0.976 & $\begin{array}{c}5.3 \text { billion } \\
( \pm 3.6 \text { billion })\end{array}$ & 1.2 \\
\hline
\end{tabular}

\subsection{AFM: SURFACE TOPOGRAPHY}

In order to determine the defect density of graphitic samples in these experiments, AFM was first utilized to replicate the process used by Patrick Unwin's group at the University of Warwick. ${ }^{49}$, 50, 229 While results were obtainable on ZYA, there was no method to discern defects on PG because the surface is substantially rougher than the length scale of defects: Ra for PG was 30.1 $\mathrm{nm}$ (Figure 1) and a single graphene step edge is $0.335 \mathrm{~nm}$, thus defects become washed out due to surface roughness. A roughness threshold of defects was postulated where roughness above e.g., $1 \mathrm{~nm}$ would be due to sample surface topography and roughness below $1 \mathrm{~nm}$ would be due to defects; however, this threshold would be arbitrary and not based on any sound reasoning. Furthermore, the necessary AFM resolution was not possible considering the sample surface topography. Therefore, it was decided to forego AFM and base defect density calculations on Raman spectroscopy data. This highlights the necessity for complimentary methods when 
performing scientific analysis such as quantifying the quality of graphite via defects: certain defects detectable by Raman are not detectable by AFM and vice versa.

Atomic force microscopy (AFM) was used to investigate topography of the graphite samples. This data was used to first understand the influence of surface roughness on wettability and then to calculate defect density of ZYA. Figure 15 shows AFM height and phase images for ZYA, PG, SPI-1, and SPI-2. As mentioned in Section 1.2 (page 2), there are salient differences between the samples. Phase images show that the surface is predominately graphite and other

material, presumably contaminants, accumulate along the step edges and defect sites. ${ }^{42,99}$

\subsubsection{Step edge density}

AFM is able to detect surface defects that cause a topographical change in the surface, thus step edges are predominately seen on smooth HOPG. The step edges are clearly observed on ZYA, SPI-1, and SPI-2 in Figure 15. ZYA is the highest quality sample and its surface is mostly basal plane with few edge defects; whereas the SPI samples have substantially more edge defects. This difference in number of step edges between ZYA and SPI samples has been previously reported by Patel et al. ${ }^{50}$ and AFM on SPI-1 presented by Ashraf et al. looks similar. ${ }^{230}$ PG has a bubbly surface - corroborating Raman and XRD data that this sample is turbostratic with poor $\mathrm{ABAB}$ layer stacking - and step edges are not clearly defined. 

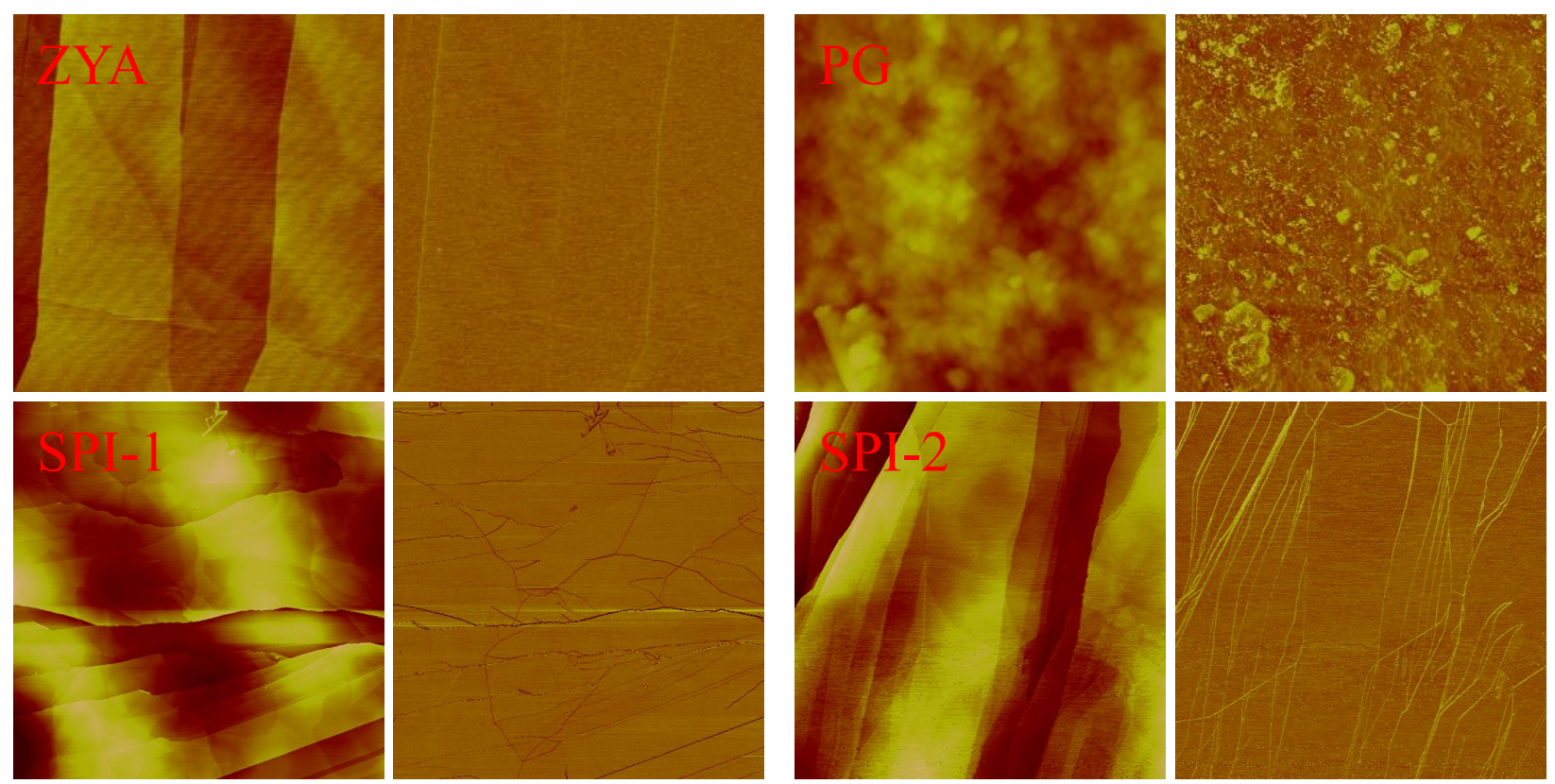

Figure 15. AFM height and phase images of ZYA, PG, SPI-1, and SPI-2. The scan size is $5 \times 5$ $\mu \mathrm{m}$ for all images. The height and phase color scale is $10 \mathrm{~nm}$ and $20^{\circ}$ for ZYA, SPI-1, and SPI2. The height and phase color scale is $500 \mathrm{~nm}$ and $50^{\circ}$ for PG. Resolution for ZYA and PG is 256 pixels while the resolution for SPI-1 and SPI-2 is 512 pixels.

Patel et al. calculated the step edge density of HOPG by measuring the length of defects and dividing by the total surface area: ${ }^{49,50,52}$

$$
\text { Step density }=\frac{\text { length of defects }}{\text { area of surface }} \quad \text { Equation } 35
$$

They calculated the mean step density of ZYA (GE Advanced Ceramics) to be $0.5 \pm 0.1$ $\mu \mathrm{m} / \mu \mathrm{m}^{2}$. Moreover, they showed that SPI-1 has nearly $3 \mathrm{x}$ as many step edges as ZYA, even though the samples are considered to be comparable (see Section 1.2 on page 2). ${ }^{18,50}$ SPI-1 has a step density of $1.5 \pm 0.2 \mu \mathrm{m} / \mu \mathrm{m}^{2}$ and SPI-2 has a step density of $2.1 \pm 0.9 \mu \mathrm{m} / \mu \mathrm{m}^{2}$. They also show that the basal surface of HOPG is electrochemically active and that the samples with fewer 
step edges (i.e., ZYA) have better electron transfer activity, behaviour that goes against prior

evidence that the basal plane is inert. ${ }^{50-52}$ The aforementioned work by Patel et al..$^{49-52}$ along with Figure 15 and Figure 16 clearly show that ZYA and SPI-1 are not of comparable quality in terms of surface topography and electrochemical activity: SPI-1 has more surface defects. Therefore, further experimental work was performed on ZYA in an effort to elucidate the wettability of pristine $\mathrm{sp}^{2}$ carbon.

The step edge density of freshly exfoliated ZYA was calculated using Equation 35 from 20 different AFM scans. The average step edge density was measured to be $0.78 \pm 0.29 \mu \mathrm{m} / \mu \mathrm{m}^{2}$ (range: 0.29 to $1.31 \mu \mathrm{m} / \mu \mathrm{m}^{2}$ ). This matches well to the result of Patel et al. ${ }^{50}$ Similar analysis can not be performed for PG because of its surface topography, thus AFM is not applicable for quantitatively comparing the defect density of ZYA and PG. Moreover, AFM does not detect point defects. Including point defects in the analysis is important since they are the primary type of intrinsic defect within the graphite samples.

\subsubsection{Defects caused by exfoliation}

Graphite is extremely stable in air and does not degrade under ambient conditions. Exfoliating the sample with adhesive tape causes strain which can cause artificially produced defects. ${ }^{46,222}$ A single exfoliation event is not expected to contribute substantially to sample degradation, thus the subjacent surface (or the freshly exfoliated surface) can be considered as pristine graphite with comparable defect density to neighboring layers. Moreover, physical exfoliation is the only method to cleave the sample without introducing other chemicals as in liquid-phase exfoliation and sonication. Uncertainty of sample quality arises once it has been exfoliated numerous times 
to only a fraction of its original thickness. A single exfoliation event may not change the surface but many exfoliation events $(>100)$ can cause the surface to appear substantially different.

It is generally advisable that an HOPG sample should be relatively new when used in experiments and should not be used once exfoliated "too many" times; however, the threshold is arbitrary. Most researchers likely are not exfoliating the same sample hundreds of times but the nature of the experiments (in this dissertation) necessitate robust repeatability. It is important to note that all experiments conducted by myself and my co-workers were taken on samples that were purchased new and once the sample got substantially thin it was replaced and no longer used for experiments. ${ }^{62,70,75-77}$ ZYA is the highest quality of HOPG and exfoliates nearly perfectly. Visually, there were no flakes or exfoliation-induced defects noticeable and all experimental testing was performed only on the visually pristine basal surface. Nevertheless, nanoscopic defects - both intrinsic and caused by exfoliation - are present on the graphite surface which are invisible to the eye.

An interesting observation made recently was that the surface quality of ZYA changed considerably with the number of exfoliations. Figure 16 shows AFM scans of ZYA taken when the sample was new (exfoliated only several times) and when the sample was less than half of its original thickness (exfoliated $>100$ times). There is a substantial difference between the surfaces and many more step edge defects can be identified on the surface which has been exfoliated many times.

This result is important for several reasons. First, there is direct evidence that exfoliating HOPG causes surface degradation. This is not entirely surprising because physical exfoliation using adhesive tape causes curvature of the graphite layers and this strain results in an uneven exfoliation and the creation of non-intrinsic surface defects. ${ }^{46,47}$ This behavior has been 
documented indirectly when studying electron transfer kinetics on HOPG. ${ }^{46}$ Moreover, Chang and Bard used scanning tunneling microscopy (STM) to image the various features caused by exfoliation of HOPG, which can cover up to $10 \%$ of the surface. ${ }^{222}$ The influence that these exfoliation-induced defects have on wettability of graphite has not been explored.

Second, the history of the sample can have implications on its performance, e.g., slower electron transfer as defect density increases, ${ }^{50}$ diminished electrochemical performance, ${ }^{52}$ poor performance as an ion beam detector, ${ }^{231}$ etc., thus history should be taken into account in experiments. Third, in situ degradation of graphitic electrodes, sensors, scaffolds, etc. can diminish the device performance over time and result in eventual failure or reduced sensitivity. Taking degradation into consideration is essential for device reliability and Figure 16 unequivocally shows that even the highest quality HOPG commercially available is subject to exfoliation induced surface degradation.

\subsubsection{Number of defects to carbon atoms}

Pertaining to this study, the WCA of ZYA was taken when the sample was new from the vendor (Figure 16a) and after being exfoliated $>100$ times (Figure 16b). Both fresh and aged WCA were the same on both surfaces indicating that the increased amount of step edge defects, caused by exfoliation, does not have an impact on macroscale surface wettability. The WCA on freshly exfoliated ZYA (a) new from the vendor and (b) after being exfoliated many times was $65.7^{\circ} \pm$ $1.2^{\circ}(\mathrm{N}=7)$ and $65.6^{\circ} \pm 1.4^{\circ}(\mathrm{N}=22)$.

This result is interesting because I initially hypothesized that the surface with more defects would have a lower WCA; however, this was not the case. The most likely explanation takes into account the number of surface defects that the water drop contacts. Raman data (Table 
10 on page 68) shows that the drop contacts 108 million defects on freshly exfoliated ZYA which was tested new from the vendor. Using the defects per carbon atom, the water drop contacted $c a .1 .8 \times 10^{14}$ carbon atoms for every defect. Therefore, even with a seemingly large amount of defects, the water drop sees mostly $\mathrm{C}-\mathrm{C}$ bonds and relatively few defects. This remains true even if the number of defects doubles or triples as what may happen when ZYA is exfoliated many times; therefore, there is no macroscopic change to surface wettability although microscopic wetting may change at local defect sites (see Section 5.3 on page 98).

Applying this result to a nanoscale water drop (opposed to a $2 \mu \mathrm{L}$ drop) may lead to interesting behaviour as the relative number of defects per carbon atom increases. In fact, there are reports showing that nanoscale wetting is more hydrophilic than macroscale wetting. ${ }^{42,99,232-}$ 234 For graphite, this behaviour may be due to the greater concentration of hydrophilic defects relative to carbon atoms.
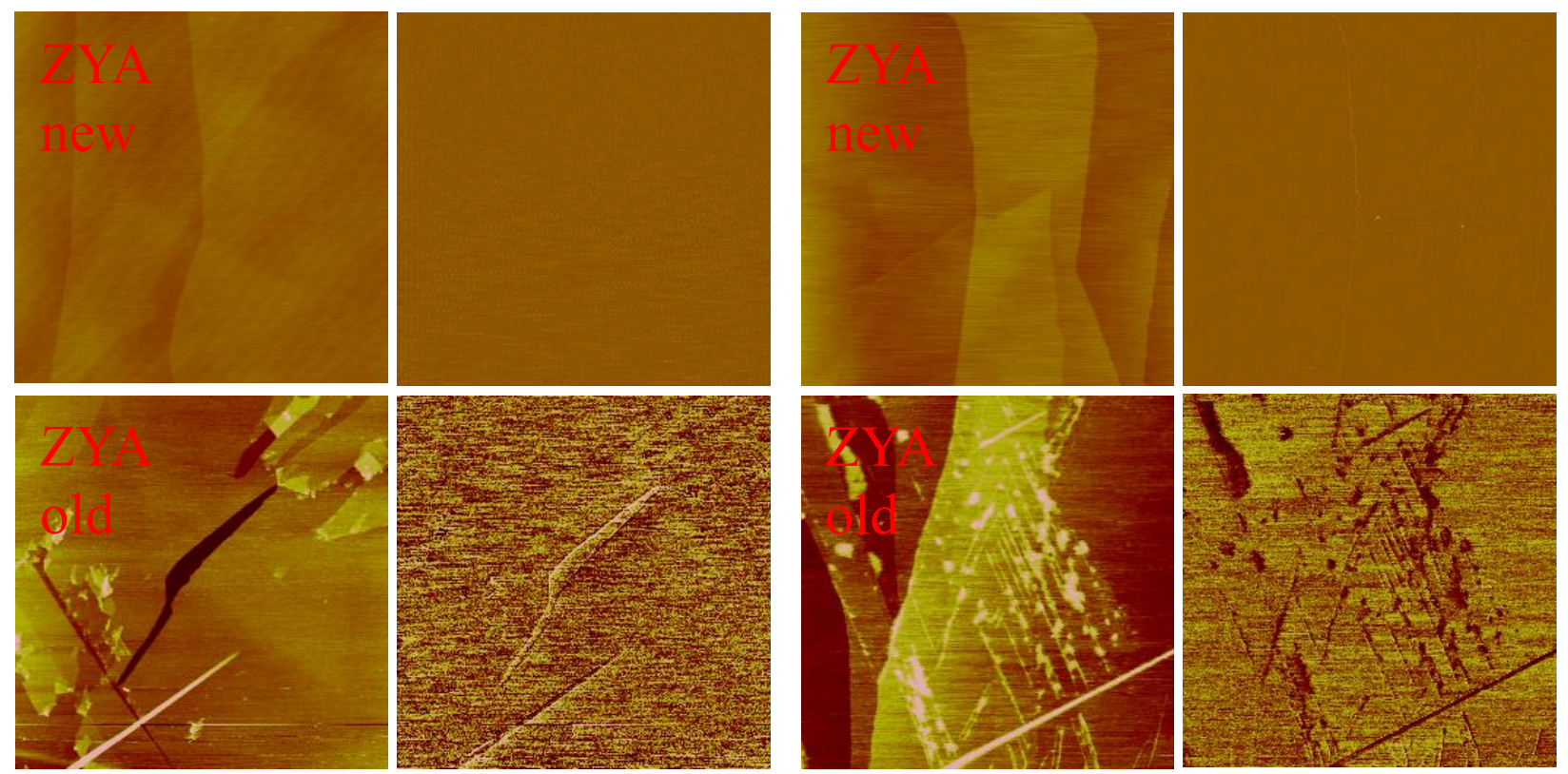

Figure 16. AFM height and phase images of ZYA. (a) Sample was new from the vendor and only exfoliated a few times. (b) The same sample after being exfoliated numerous times. The number of exfoliation events was not counted. 


\subsection{INTRINSIC WATER WETTABILITY OF GRAPHITE}

\subsection{STATIC WATER CONTACT ANGLE}

The water contact angle of freshly exfoliated graphite was evaluated using a $2 \mu \mathrm{L}$ water drop. 1inch scotch tape was used to exfoliate the HOPG samples as detailed in our previous work. ${ }^{62,70,}$ 75-77 PG was exfoliated by carefully cleaving with a razor blade. Section 5.4 (page 114) discusses the effect that method of exfoliation - adhesive tape versus razor - has on the graphite surface. WCA on fresh ZYA is the same whether the sample is exfoliated by adhesive tape or razor; therefore, method of exfoliation has no influence on the intrinsic WCA and data obtained by either method can be directly compared.

WCA data on fresh HOPG and PG samples is shown in Figure 17 and Table 11. The first observation is that all four HOPG samples, regardless of quality, have similar WCA on the fresh surface: average WCA of the four HOPG samples is $65.3^{\circ} \pm 1.3^{\circ}(\mathrm{N}=71)$. This is analogous to WCA on freshly exfoliated SPI-2 HOPG previously reported by our group (see Section 2.4 on page 18). ${ }^{76,77}$ Interestingly, wettability is not affected by HOPG quality even though the lower quality samples (ZYH and SPI-2) have more step edges, and likely more point defects, than the highest quality samples (ZYA and SPI-1). Figure 1 (page 9) shows optical and AFM images of the graphite samples. This supports the concept that all four HOPG samples are high quality and vary only in the number of step edges and size of crystallite domains. Furthermore, sample 
quality is quantified by mosaicity, which is a bulk parameter, while wetting is only sensitive to the sample surface. This data shows that wetting is not a function of mosaicity and is affected by surface chemistry at the uppermost layers.

WCA of freshly exfoliated PG is $47.4^{\circ} \pm 2.7^{\circ}$ which is substantially lower than HOPG. Wenzel and Cassie-Baxter analysis was performed to elucidate the effects of surface roughness and chemical heterogeneity on the observed WCA. The influence of surface defects was investigated to determine if the observed mild hydrophilicity of fresh graphite is intrinsic to $\mathrm{sp}^{2}$ hybridized carbon or if the hydrophilic behaviour is due to surface defects. The subsequent analysis seeks to clarify the relationship between defect density and water wettability.

WCA for all of the graphite samples was $c a .90^{\circ}$ after aging in ambient air for 1-2 days. Increase of WCA is attributed to adsorption of adventitious hydrocarbons in the surrounding air. The graphite surface is initially mildly hydrophilic with high surface energy and attracts hydrocarbons which adsorb onto the fresh surface, "shield" the graphite, and cause the sample to appear hydrophobic with a WCA about $90^{\circ}$. Essentially, the WCA test is probing the hydrophobic hydrocarbon layer opposed to the subjacent graphite. ${ }^{71,235}$ This mechanism is described in Section 2.2 (page 12) and in our previous work on graphene and HOPG. ${ }^{75-77}$

Mangolini et al. used near-edge x-ray absorption fine structure (NEXAFS) spectroscopy and XPS to investigate the thickness and chemical nature of the adsorbed contamination layer on diamond and amorphous carbon. They reported that the contaminant layer has a thickness of 0.6 $\pm 0.2 \mathrm{~nm}$ and consists of $19 \pm 3 \% \mathrm{sp}^{2}$ carbon. Moreover, they report the ratio of adsorbed oxygen-to-carbon to be $0.11 \pm 0.02 .{ }^{236}$ These findings are significant because they probe the contaminant layer and begin to elucidate the type of hydrocarbons adsorbing onto the graphite surface. Our previous work showed the hydrocarbon thickness on HOPG to be $c a .0 .50 \mathrm{~nm}$ 
(Figure 3 on page 15) using ellipsometry which is similar to the $0.6 \mathrm{~nm}$ obtained by Mangolini. ${ }^{76}$, 236

This temporal dependence of WCA has been well documented since 2013 with numerous studies showing that fresh graphite, graphene, TMDC, and REO surfaces are intrinsically more hydrophilic than expected. ${ }^{62,70,71,76}$ The concept of adventitious hydrocarbon contamination is well documented on metals ${ }^{35-40}$ but only recently has been applied to graphitic surfaces. Table 12 shows published data (as of March 31, 2016) which experimentally report that fresh graphite is mildly hydrophilic. No researchers have yet published data refuting the intrinsic hydrophilicity of these materials since 2013. The ongoing debate includes the wetting transparency effect for 2D materials, ${ }^{111,123,237-239}$ the cause of intrinsic mild hydrophilicity, ${ }^{76,77}$ and the degree in which hydrocarbon and water adsorption influence wettability. ${ }^{63,64}$

Importantly, this concept is becoming better understood as more researchers realize the importance of hydrocarbon contamination on graphitic surfaces and take into account exposure time in air when conducting experiments and fabricating devices. Work by Tadros, Hu, and Adamson in 1974 was the first to suggest that isotropic carbon (polished, cleaned with toluene and acetone, then degassed for 10-15 hours at room temperature) had a WCA of $63^{\circ} .{ }^{32}$ Subsequent studies reported by Malcolm Schrader published in 1975 and 1980 further show that the WCA of graphite may be $35^{\circ},{ }^{33,34}$ however, experimental concerns were presented as discussed in Section 2.1. ${ }^{92}$ Nevertheless, until 2013, only two reports published in 1999 and 2011 mentioned the hydrophilicity of graphite although they did not explicitly study the wettability of the fresh surface. ${ }^{99,100}$ Within the past two years, researchers have begun taking hydrocarbon contamination into consideration and the WCA of fresh surfaces is being used for computer simulations and modeling. Most recently, Ramos-Alvarado et al. used the water 
contact angle of clean graphitic carbon for calibrating the water-carbon interaction potential for molecular dynamic simulations investigating wetting transparency on silicon. ${ }^{240}$

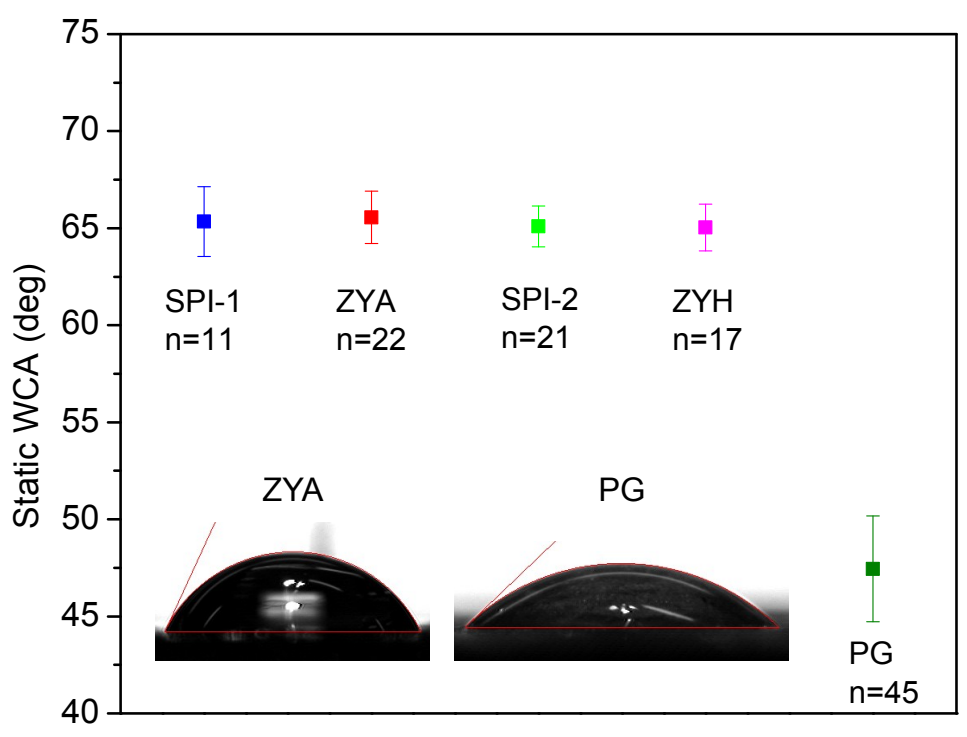

Figure 17. Static WCA of freshly exfoliated graphite.

Table 11. Static WCA of fresh graphitic surface exfoliated with tape and razor. N indicates number of tests.

\begin{tabular}{|c|c|c|c|c|}
\hline \multirow{2}{*}{} & \multicolumn{2}{|c|}{ Tape Exfoliated } & \multicolumn{2}{c|}{ Razor Exfoliated } \\
\cline { 2 - 5 } & WCA & $\mathrm{N}$ & WCA & $\mathrm{N}$ \\
\hline ZYA & $65.6^{\circ} \pm 1.4^{\circ}$ & 22 & $66.4^{\circ} \pm 1.9^{\circ}$ & 20 \\
\hline SPI-1 & $65.3^{\circ} \pm 1.8^{\circ}$ & 11 & -- & -- \\
\hline SPI-2 & $65.1^{\circ} \pm 1.1^{\circ}$ & 21 & -- & -- \\
\hline SPI-2 Ref. 76 & $64.4^{\circ} \pm 2.9^{\circ}$ & 7 & -- & -- \\
\hline ZYH & $65.0^{\circ} \pm 1.2^{\circ}$ & 17 & -- & -- \\
\hline PG & -- & -- & $47.4^{\circ} \pm 2.7^{\circ}$ & 45 \\
\hline
\end{tabular}


Table 12. Literature data on the intrinsic hydrophilicity of graphite. Data published as of March $31,2016$.

\begin{tabular}{|c|c|c|c|c|}
\hline Author & Fresh WCA & Sample & Method & Notes \\
\hline Tadros $1974^{32}$ & $63^{\circ}-65^{\circ}$ & Pyrolytic carbon & Captive bubble & -- \\
\hline Schrader $1975^{33}$ & $\begin{array}{c}50^{\circ}-80^{\circ \mathrm{a}} \\
35^{\circ} \mathrm{b}\end{array}$ & Oriented graphite & $\begin{array}{l}{ }^{\mathrm{a}, \mathrm{b}} \text { Sessile drop } \\
{ }^{\mathrm{b}} \text { Cold finger }\end{array}$ & $\begin{array}{c}{ }^{\mathrm{a}} \text { Air } \\
{ }^{\mathrm{b}} \text { Heated vacuum }\end{array}$ \\
\hline Schrader $1980^{34}$ & $\begin{array}{l}65^{\circ \mathrm{a}} \\
38^{\circ}\end{array}$ & ZYB graphite & $\begin{array}{l}\text { Sessile drop by cold } \\
\text { finger }\end{array}$ & $\begin{array}{l}{ }^{\mathrm{a}} \mathrm{RT} \text { vacuum } \\
{ }^{\mathrm{b}} \text { Heated vacuum }\end{array}$ \\
\hline Luna $1999^{100}$ & $30^{\circ}$ & Graphite & $\begin{array}{l}\text { Scanning force } \\
\text { microscopy }\end{array}$ & Nanoscale drop \\
\hline Cao $2011^{99}$ & $10^{\circ}$ & HOPG & Nanodrops & $\begin{array}{l}\text { Graphene } \\
\text { templating }\end{array}$ \\
\hline Li $2013^{77}$ & $64.4^{\circ}$ & SPI-2 & Sessile drop & -- \\
\hline Ashraf $2014^{230}$ & $53^{\circ}$ & SPI-1 & Sessile drop & $\begin{array}{c}45^{\circ} \text { cleaved in UHP } \\
\text { argon }\end{array}$ \\
\hline Kozbial $2014^{76}$ & $64.4^{\circ}$ & SPI-2 & Sessile drop & -- \\
\hline Kozbial $2014^{75}$ & $64.6^{\circ}$ & SPI-2 & Sessile drop & -- \\
\hline Amadei $2014^{64}$ & $68^{\circ}$ & HOPG & Sessile drop & -- \\
\hline Wei $2015^{241}$ & $61^{\circ}$ & $\begin{array}{c}\text { ZYH } \\
\text { (Mikro Masch) }\end{array}$ & Sessile drop & $\begin{array}{l}\text { Edge surface is } \\
\text { more hydrophilic } \\
\text { than basal plane }\end{array}$ \\
\hline Marbou $2015^{242}$ & $\begin{array}{l}{ }^{\mathrm{a}} 50^{\circ} \\
\mathrm{b} 35^{\circ}\end{array}$ & ZYB (Ted Pella) & ${ }^{\mathrm{a}, \mathrm{b}}$ Microscopic; ESEM & $\begin{array}{l}{ }^{\mathrm{a}} \text { Fresh; vacuum } \\
{ }^{\mathrm{b}} \text { Heated vacuum }\end{array}$ \\
\hline $\operatorname{Li} 2015^{62}$ & $65^{\circ}$ & SPI-2 & Sessile drop & - \\
\hline Aria $2016^{243}$ & $69^{\circ}$ & HOPG & Sessile drop & $\begin{array}{c}\text { Tested after } 30 \mathrm{~min} \\
\text { in air }\end{array}$ \\
\hline
\end{tabular}




\subsection{DYNAMIC WCA AND HYSTERESIS}

\subsubsection{Theory and background of hysteresis}

Static contact angles, e.g., sessile drop, measure equilibrium between the solid surface and liquid. Dynamic contact angles provide further information on surface wettability as the liquid drop advances onto an unwet surface (advancing water contact angle; $\mathrm{WCA}_{\mathrm{a}}$ ) and recedes onto a wet surface (receding water contact angle; $\mathrm{WCA}_{\mathrm{r}}$ ).

Contact angle hysteresis $\left(\theta_{\mathrm{H}}\right)$ is the difference between advancing and receding contact angle and has traditionally been used to describe the barrier to contact line movement, i.e., surface roughness and heterogeneity: ${ }^{244}$

$$
\begin{array}{cr}
\theta_{H}=\theta_{a}-\theta_{r} & \text { Equation } 36 \\
W C A_{H}=W C A_{a}-W C A_{r} & \text { Equation } 37
\end{array}
$$

Hysteresis was originally thought to indicate roughness and chemical heterogeneity of a solid surface. The Young's contact angle $\left(\theta_{\mathrm{Y}}\right)$ was theorized to be the unobtainable contact angle on a perfectly smooth and homogeneous surface and any deviation from $\theta_{\mathrm{Y}}$ is due to roughness and chemical heterogeneity. ${ }^{29,141,172}$ The theory explains that a surface consisting of two (or more) individually homogeneous surfaces can be thought of as a composite surface of the two individual surfaces. In other words, surface 1 having islands of surface 2 can be modeled as the average of the two surfaces: 


$$
\theta_{\text {composite }}=\frac{1}{n} \sum_{i}^{n} \theta_{i} \quad \text { Equation } 38
$$

where $\theta_{1}$ is the contact angle of surface $1, \theta_{2}$ is the contact angle of surface 2 , etc.

An ideal perfectly smooth homogeneous surface would have $\theta_{\mathrm{H}}$ of $0^{\circ}$ and $\theta_{\mathrm{H}}$ increases with roughness and heterogeneity. ${ }^{29}$ While this theory of hysteresis has generally been well accepted, various phenomena surrounding the dynamics of advancing and receding contact angle behaviour have remained unsatisfactorily explained and several researchers have postulated alternative causes of hysteresis. $^{245-248}$

Substantial information is obtained from dynamic WCA measurements which allow for elucidation of wetting behaviour. $\mathrm{WCA}_{\mathrm{a}}$ reflects the tendency of a previously unwet (dry) surface to attract water; whereas, $\mathrm{WCA}_{\mathrm{r}}$ reflects the tendency of the surface to remain wetted. The static WCA can theoretically take any value between $\mathrm{WCA}_{\mathrm{a}}$ and $\mathrm{WCA}_{\mathrm{r}}$, although it often falls at a particular value that is repeatable for the same material (with the same amount of roughness and chemical heterogeneity). A small difference between $\mathrm{WCA}_{\mathrm{s}}$ and $\mathrm{WCA}_{\mathrm{a}}$ (or $\left.\mathrm{WCA}_{\mathrm{r}}\right)$ indicates that the surface is mostly uniform and wettability of the composite surface (e.g., basal plane and defects) is similar to the pristine surface. Contrarily, a large difference between $\mathrm{WCA}_{\mathrm{s}}$ and $\mathrm{WCA}_{\mathrm{a}}$ (or $\mathrm{WCA}_{\mathrm{r}}$ ) indicates that chemical heterogeneity (e.g., defects) has a salient impact on wettability. ${ }^{246,248-252}$ The Wenzel and Cassie-Baxter models are used to help elucidate the influence of surface roughness and chemical heterogeneity on measuring the intrinsic WCA. This is discussed in Section 5.3 (page 98).

Nearly all of the most recent work done to elucidate the causes of hysteresis have sought to explain phenomena surrounding superhydrophobic surfaces; therefore, they use micropatterned surfaces with a repetitive pillar array. ${ }^{251,253-256}$ The pillars are typically on the 
micron scale and range from a few to several microns in height, width, and spacing. When a water drop is placed on this micropatterned surface, it will interact with a composite surface of (1) the pillars and (2) air pockets trapped between the pillars. This situation has led researchers to propose alternative models - to the Wenzel and Cassie-Baxter models - in order to explain wetting behaviour on superhydrophobic surfaces. The Wenzel model is discussed in Section 5.3.1 (page 102) and the Cassie-Baxter model is discussed in Section 5.3.2 (page 104).

Defects on graphite are different from micropatterned arrays in many ways. The most salient difference is the size scale: defects are on the scale of nanometers (or fractions of a nanometer) whereas micropatterned pillars are micrometer sized (1000s of nanometers). This creates questions surrounding the applicability of theory formulated using micropatterned surfaces. Moreover, air pockets are very unlikely to reside between the graphite surface and liquid because the graphite surface (with defects) is significantly smoother than a micropatterned array of pillars. This leaves the possibility that equations formulated for micropatterned pillar arrays are (a) not relevant for graphite, (b) relevant for graphite, or (c) need amended for graphite. The subsequent analysis shows that currently available models - specifically the model proposed by Cassie and Baxter in 1944 - adequately describes wetting phenomena when contact line pinning is negligible due to low defect density; however, the model fails when pinning distorts the contact line.

Larsen and Taboryski studied the behaviour of hysteresis on chemically heterogeneous surfaces and showed that the contact line is distorted by defects and behaviour of the advancing and receding contact angle is dependent on surface wetting compared to defect wetting. Their 2009 report investigated the wetting mechanism using a patterned hydrophilic substrate with hydrophobic defects (Figure 18). ${ }^{253}$ This situation is opposite that encountered on fresh graphite 
(mildly hydrophilic surface with defects that are more hydrophilic) although the wetting mechanism remains similar for both situations. A quick glance at Figure 18a shows that the water contact line is irregular and not symmetrical. Hydrophobic pillars repel water while the hydrophilic substrate attracts water. This creates competing interactions between water-substrate and water-pillars which manifest as pinning of the contact line. Pinning is more clearly observed in Figure $18 \mathrm{~b}$ where water advances onto the substrate between pillars but is pinned from advancing by the pillars. This mechanism of pinning also occurs for hydrophilic defects on a more hydrophobic surface (e.g., graphite) and is critical for explaining wetting behaviour of chemically heterogeneous surfaces such as graphite.

(a)

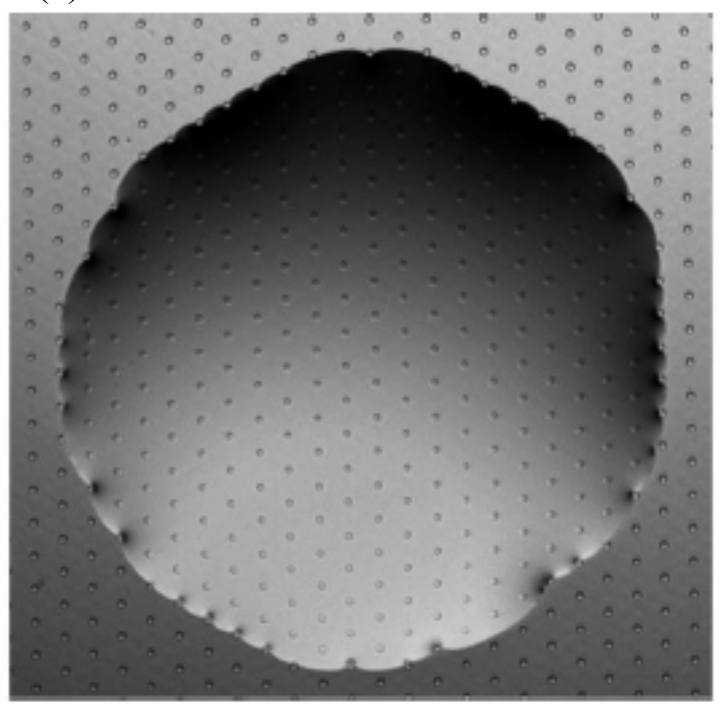

(b)

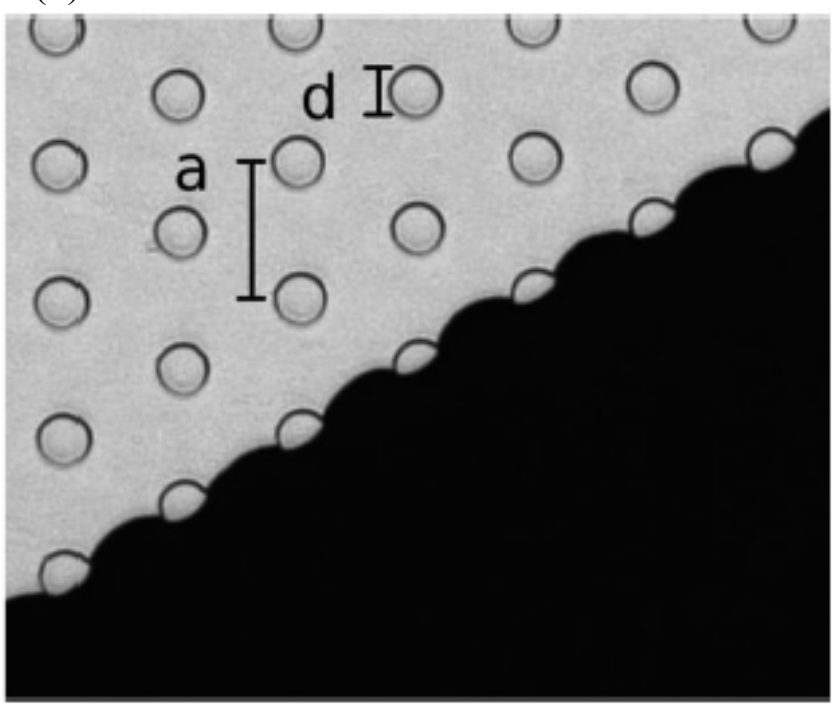

Figure 18. Schematic of contact line pinning for a water drop on a hydrophilic surface with hydrophobic defects (circles). Pinning of the contact line is conspicuously observed. Adapted with permission from Larsen, S. T.; Taboryski, R. A Cassie-Like Law Using Triple Phase Boundary Line Fractions for Faceted Drops on Chemically Heterogeneous Surfaces. Langmuir 2009, 25, 1282-1284. Copyright 2009 American Chemical Society. ${ }^{253}$ 
Raj et al. followed up on this wetting behaviour and published a report on the mechanism of chemically heterogeneous wetting in 2012 . They showed that the contact line is distorted along defect sites and behaviour of the advancing and receding contact angle is dependent upon hydrophobicity/hydrophilicity of the surface compared to the defects. ${ }^{251}$ Results of their investigation are shown in Figure 19. First, they compare the pinning theory to the CassieBaxter model and show that ignoring pinning effects will cause the model to inadequately describe real wetting behaviour. The lower left images depict contact line pinning of a receding drop on $\mathrm{SiO}_{2}$ with defects that are more hydrophilic than the substrate. ${ }^{251}$ The data shows that as the difference between defect and substrate hydrophobicity/hydrophilicity increase, the effect of pinning becomes more significant. I.e., a slightly chemically heterogeneous will behave more like a homogeneous surface than a significantly chemically heterogeneous surface. ${ }^{251,253}$

In relation to the model, graphite can be considered to be mildly hydrophilic with defects that are more hydrophilic. Thus it can be modeled as in Figure 19 (top right) where the white area is graphite and the black circles are defects. The fraction of the contact line on hydrophilic defects (f) and the area fraction of hydrophilic defects (sf) can be calculated where D is the defect diameter and $\mathrm{L}$ is the pitch. ${ }^{251}$ :

$$
\begin{array}{cr}
L=L_{d}+2(\text { radius of defect }) & \text { Equation } 39 \\
f=\frac{D}{L} & \text { Equation } 40 \\
s f=\frac{\pi D^{2}}{4 L^{2}} & \text { Equation } 41
\end{array}
$$


Heterogeneous surface with square array of hydrophilic circular defects
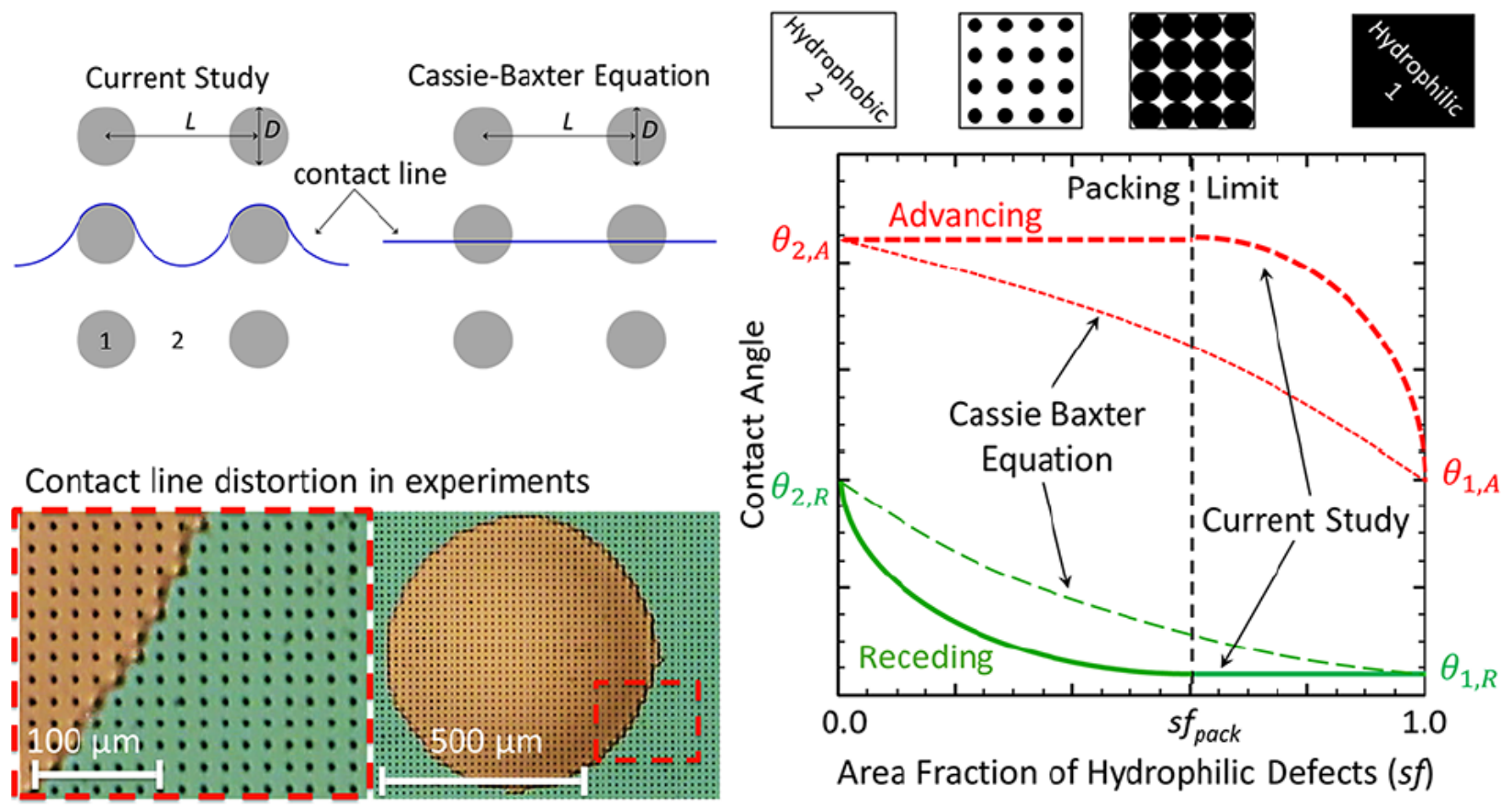

Figure 19. Theory of contact angle hysteresis for a liquid on a hydrophobic surface with hydrophilic defects. The upper left image shows the effect of contact line pinning at defect sites (circles) in relation to the Cassie-Baxter model which does not take pinning into consideration.

The lower left shows time-lapse microscope images of a receding drop on $\mathrm{SiO}_{2}$ with more hydrophilic defects (black dots). Pinning of the contact line is conspicuously observed. The right image shows how the advancing and receding contact angle behaves as a function of defect density. Adapted with permission from Raj, R.; Enright, R.; Zhu, Y.; Adera, S.; Wang, E. N. Unified Model for Contact Angle Hysteresis on Heterogeneous and Superhydrophobic Surfaces. Langmuir 2012, 28, 15777-15788. Copyright 2012 American Chemical Society. ${ }^{251}$

The size of an individual defect is proportionally related to the surface defect density (i.e., larger defects increase defect density and decrease basal surface). The radius of a single defect $\left(r_{d}\right)$ can be estimated as the covalent radius of a carbon atom $(73 \pm 2 \mathrm{pm}),{ }^{257,258}$ which is the lower limit of $r_{d}$. In order to not underestimate the size of point defects, $r_{d}$ will be estimated to be $1 \mathrm{~nm}$ (see Section 5.3.2.1 on page 109) and the defect diameter (D) equals $2 \mathrm{~nm}$. The pitch (L) can then be calculated using the distance between defects $\left(\mathrm{L}_{\mathrm{d}}\right)$ from Raman (Table 9 on page 
63); therefore, the area fraction of defects (sf) can be calculated and results are shown in Table 13.

Table 13. Defect characterization for exfoliated ZYA and PG. Data is based on the distance between defects $\left(\mathrm{L}_{\mathrm{d}}\right)$ determined from the graphite Raman spectra and an assumption of the defect radius $\left(\mathrm{r}_{\mathrm{d}}\right)$.

\begin{tabular}{|c|c|c|}
\hline & ZYA & PG \\
\hline L (nm) & 118 & 15 \\
\hline D (nm) & 2 & 2 \\
\hline f & 0.0169 & 0.1333 \\
\hline sf & 0.0002 & 0.0140 \\
\hline
\end{tabular}

The graph in Figure 19 shows the relationship between advancing/receding WCA and area fraction of defects (sf). ${ }^{251}$ Both ZYA and PG are at the very leftmost part of the graph (even when defect size is quite large) indicating that defects have no effect on the advancing WCA and the receding WCA is near its maximum. Thus, increasing the amount of defects - up to its packing limit - would have negligible effect on advancing WCA and cause receding WCA to decrease.

Several studies unequivocally show that receding WCA is a manifestation of liquid pinning due to defects, i.e., the liquid prefers to remain in contact with hydrophilic defect sites instead of retract towards the hydrophobic surface, thus WCA decreases as water is withdrawn until intermolecular forces within the liquid are strong enough to overcome the hydrophilic attraction (and the contact line is pulled inward). Conversely, advancing WCA is a manifestation 
of liquid interaction with the interconnected hydrophobic surface, i.e., the liquid prefers to remain in contact with hydrophilic defects at its current position instead of advance onto the hydrophobic surface, thus contact angle increases as water is added until intermolecular forces within the liquid are strong enough to overcome the hydrophilic attraction (and the contact line is pushed outward). Furthermore, the advancing contact angle is independent of defects until the packing maximum $\left(\mathrm{sf}_{\max }\right)$ is reached. Essentially, the advancing contact line mechanism is opposite the receding contact line mechanism and this behaviour reverses when the surface is hydrophilic and the defects are hydrophobic. ${ }^{27,250,251,253,259}$

\subsubsection{Current understanding of static and dynamic contact angles}

Theory of surface wetting has significantly advanced since Thomas Young first wrote about interfacial surface tension in $1805 .{ }^{141}$ While the concept of wetting may initially seem rather facile, its mechanism has proven complicated and a thorough understanding is still not available. Nevertheless, previous studies have provided valuable insight into wetting behaviour and recent technology advances have allowed fabrication of micropatterned arrays which has substantially advanced understanding of the mechanisms behind advancing and receding contact angles. Wetting is a function of the contact line as shown by Extrand ${ }^{260}$ and Gao and McCarthy; ${ }^{261}$ therefore, the mechanism for static and advancing/receding contact angle will be analyzed based only on the contact line and not contact area (see Figure 24 on page 99).

Advancing or receding contact angle can not be evaluated unless a static drop is first placed on the sample. Therefore, analysis of wetting begins with phenomena of the static water drop followed by the advancing then receding water drop. The sample surface is initially unwet

(dry). When the water drop is placed onto the surface, the water will contact both surface and 
defects indiscriminately. The water has no choice but to interact with both the surface and defects. This situation will look like Figure 20a. The experimentally observed WCA can now be defined as the thermodynamic equilibrium of water, defects, and substrate along the contact line. $251,253,262$

Adding liquid to the water drop will cause WCA to increase while maintaining a constant diameter. This is due to pinning of the contact line as shown in in Figure 20b. For the case of graphite, the graphite surface restricts water from advancing further onto the hydrophobic surface. As liquid is continually added to the drop, a critical point will be reached where advancing onto the hydrophobic surface is more energetically favorable than further increasing WCA. At this advancing critical point, the drop is at quasi-equilibrium where further adding liquid will cause the drop to reconfigure. This point is taken as $\theta_{\mathrm{a}}$, the WCA immediately before drop diameter increases. This situation is depicted in Figure 20c.

For a hydrophobic surface with hydrophilic defects, Raj et al. showed that advancing WCA is not a function of defect density up to the defect packing limit. ${ }^{251}$ This is consistent with the aforementioned mechanism since water maximizes its interaction with hydrophilic defects and minimizes its interaction with the hydrophobic surface. Increasing defect density - up to its packing limit - will not cause $\mathrm{WCA}_{\mathrm{a}}$ to change because the advancing motion is restricted by the hydrophobic surface. Therefore, $\mathrm{WCA}_{\mathrm{a}}$ represents the surface wettability sans defects. 
(a)
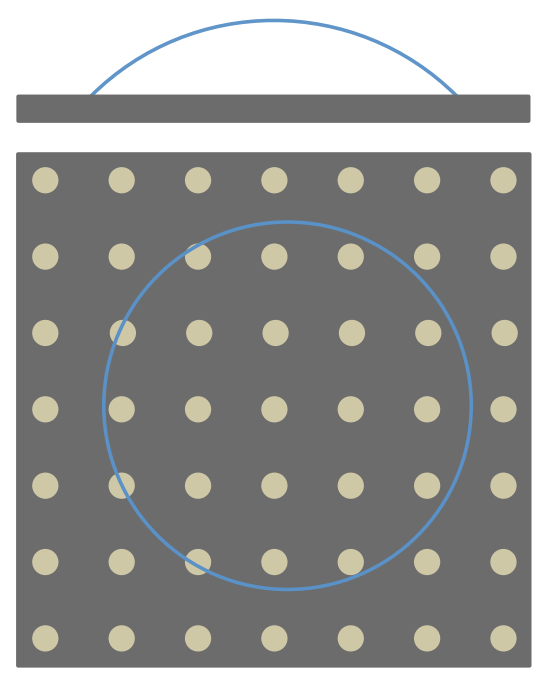

(b)
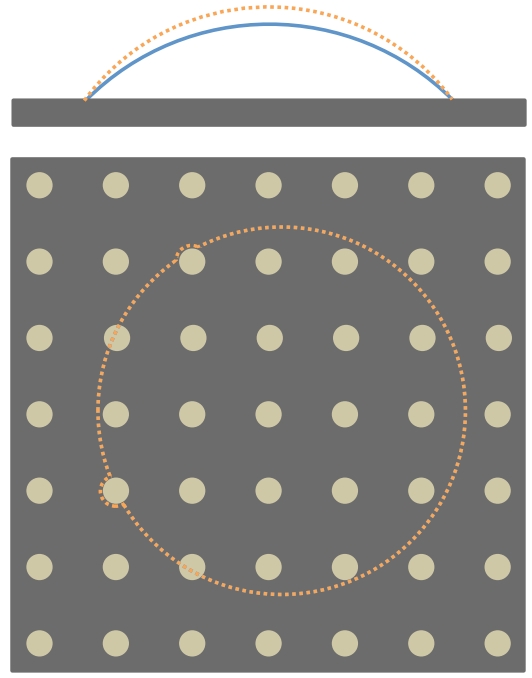

(c)
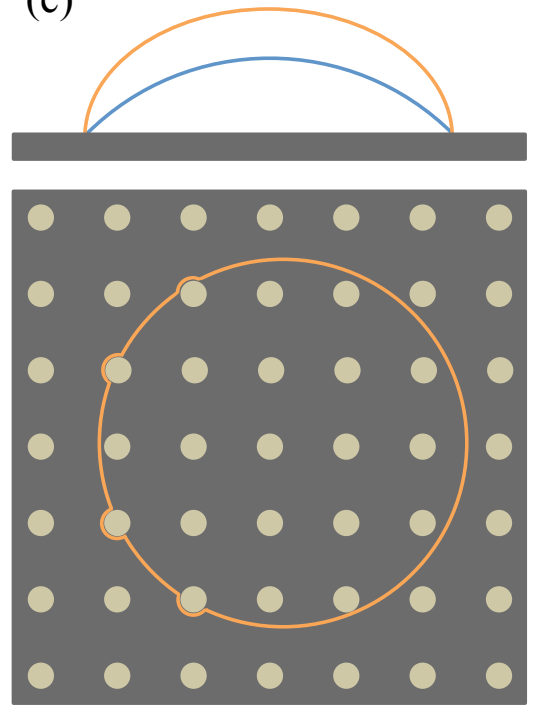

Figure 20. Advancing contact angle mechanism. The upper figures are a side view and the lower figures are an overhead view. The colored lines represent the water contact line. The black area represents the hydrophobic surface and the circles represent hydrophilic defects. (a) Static water drop placed on the sample surface. The contact line interacts with both surface and defects. (b) Intermediate state: the drop begins to advance onto the defects as liquid is added. The contact line becomes pinned and contact angle increases. (c) Advancing contact angle: the contact line remains pinned by the hydrophobic surface and does not advance as water is added to the drop. The drop advances onto the unwet surface once the advancing critical point is reached.

Receding contact angle is exactly the opposite mechanism of advancing contact angle. Removing liquid from the water drop will cause the WCA to first decrease while maintaining a constant diameter. This is due to pinning of the contact line by defect sites as shown in Figure 21b. For the case of graphite, hydrophilic defects restrict water from receding onto the hydrophobic graphite. As liquid is continually removed from the drop, a critical point will be reached where receding onto the hydrophobic surface is more energetically favorable than further decreasing WCA. At this receding critical point, the drop is at quasi-equilibrium where further removing liquid will cause the drop to reconfigure. This point is taken as $\theta_{\mathrm{r}}$, the WCA immediately before drop diameter decreases. This situation is depicted in Figure 21c. 
For a hydrophobic surface with hydrophilic defects, Raj et al. shows that receding WCA is a strong function of defect density up to the defect packing limit. ${ }^{251}$ This is consistent with the aforementioned theory since water maximizes its interaction with hydrophilic defects and minimizes its interaction with the hydrophobic surface. Increasing defect density - up to its packing limit - will cause $\mathrm{WCA}_{\mathrm{r}}$ to asymptotically decrease because the receding motion is restricted by the hydrophilic defects. Therefore, $\mathrm{WCA}_{\mathrm{r}}$ represents the defect wettability when defects are at or above their packing limit (which is not the case for fresh graphite).

(a)
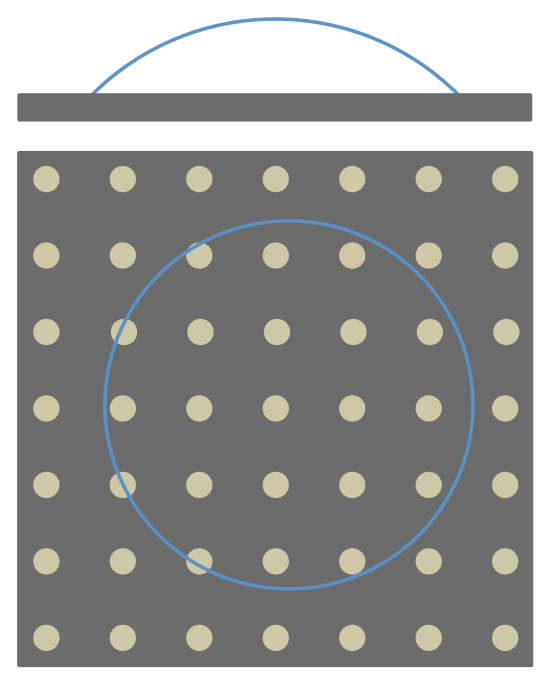

(b)
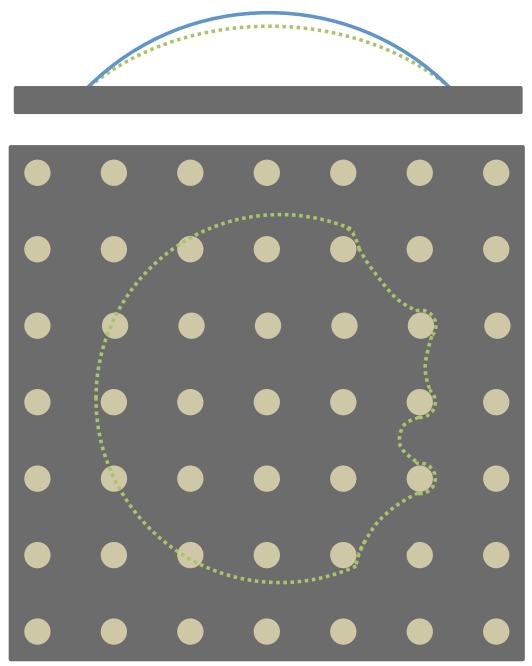

(c)
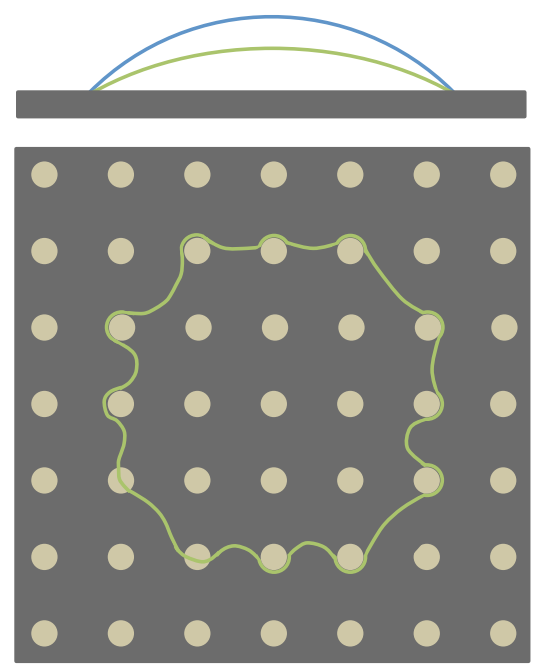

Figure 21. Receding contact angle mechanism. The upper figures are a side view and the lower figures are an overhead view. The colored lines represent the water contact line. The black area represents the hydrophobic surface and the circles represent hydrophilic defects. (a) Static water drop placed on the sample surface. (b) Intermediate state: the drop begins to recede towards the defects as liquid is withdrawn. The contact line becomes pinned and contact angle decreases. (c) Receding contact angle: the contact line remains pinned by hydrophilic defects and does not recede as water is withdrawn from the drop. Pinning causes the tortuous contact line. The drop recedes onto the wet surface once the receding critical point is reached. 
The aforementioned dynamic contact angle mechanisms allow for the wettability of $\mathrm{sp}^{2}$ hybridized carbon to be evaluated based on the advancing WCA and the wettability of defects to be estimated based on the receding WCA. Because defect density is very low (below its packing limit) for both ZYA and PG, the advancing WCA corresponds to WCA of the sample surface (i.e., $\mathrm{sp}^{2}$ carbon sans defects); whereas the receding WCA corresponds to the WCA of defects at the present defect density. Increasing defect density (up to its packing limit) will asymptotically decrease receding WCA and reflect wettability of the defects as shown in Figure 19.

\subsubsection{Dynamic WCA on graphite}

Advancing WCA measurements were taken by recording continuous addition of water to a sessile drop $(2 \mu \mathrm{L})$ for 5-8 seconds. Receding WCA measurements were taken by withdrawing liquid from the same sessile drop for 8 seconds. The moment when the contact angle is maximum (minimum) is taken as the advancing (receding) contact angle: $\mathrm{WCA}_{\mathrm{a}}$ is the moment right before the drop width increases and $\mathrm{WCA}_{\mathrm{r}}$ is the moment right before the drop width decreases. Advancing and receding WCA was taken on freshly exfoliated HOPG and PG samples to elucidate how wetting behaviour changes as the water drop is moved to a previously wet and unwet surface. Figure 22 shows dynamic WCA data for the graphite samples and Figure 23 shows hysteresis. The data is tabulated in Table 14. 


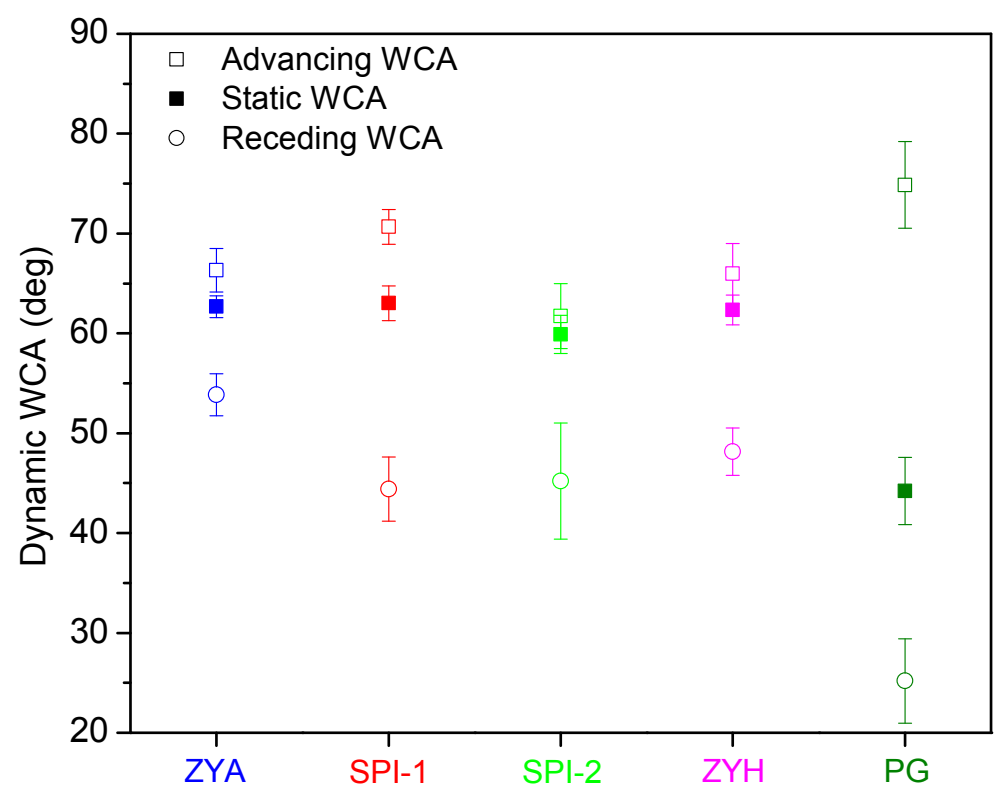

Figure 22. Dynamic WCA of freshly exfoliated graphite. Data is tabulated in Table 14 and presented as average ( \pm standard deviation).

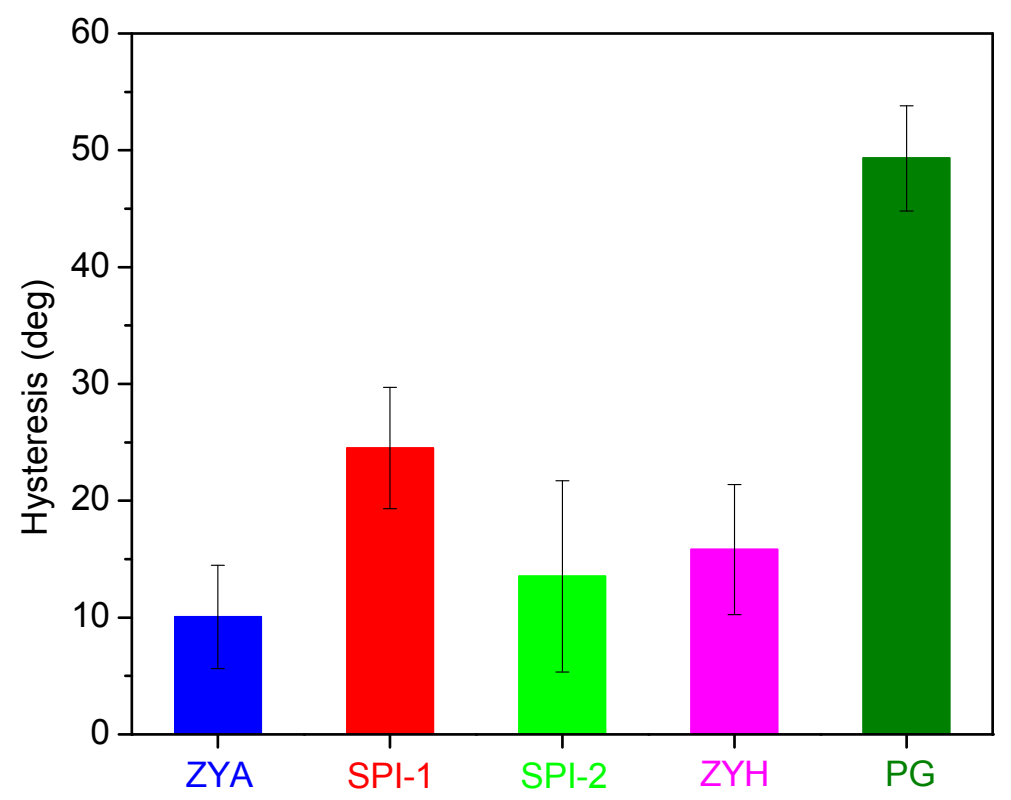

Figure 23. Hysteresis of freshly exfoliated graphite. Data presented as average $( \pm$ standard deviation). 
Table 14. Dynamic WCA and hysteresis of graphite samples. Data presented as average $( \pm$ standard deviation). $\mathrm{N}$ indicates the number of tests.

\begin{tabular}{|c|c|c|c|c|c|}
\hline & $\begin{array}{c}\text { Advancing } \\
\text { WCA }\left(\theta_{\mathrm{a}}\right)\end{array}$ & $\begin{array}{c}\text { Static WCA } \\
\left(\theta_{\mathrm{s}}\right)\end{array}$ & $\begin{array}{c}\text { Receding } \\
\text { WCA }\left(\theta_{\mathrm{r}}\right)\end{array}$ & $\begin{array}{c}\text { Hysteresis } \\
\left(\theta_{\mathrm{H}}\right)\end{array}$ & $\mathrm{N}$ \\
\hline $\mathrm{ZYA}$ & $66.3^{\circ}\left( \pm 2.2^{\circ}\right)$ & $65.7^{\circ}\left( \pm 1.2^{\circ}\right)$ & $56.3^{\circ}\left( \pm 2.2^{\circ}\right)$ & $10.1^{\circ}\left( \pm 3.2^{\circ}\right)$ & 7 \\
\hline $\mathrm{SPI}-1(\mathrm{ZYA})$ & $70.7^{\circ}\left( \pm 1.7^{\circ}\right)$ & $66.1^{\circ}\left( \pm 1.9^{\circ}\right)$ & $46.2^{\circ}\left( \pm 3.4^{\circ}\right)$ & $24.5^{\circ}\left( \pm 3.4^{\circ}\right)$ & 7 \\
\hline $\mathrm{SPI}-2(\mathrm{ZYB})$ & $61.7^{\circ}\left( \pm 3.2^{\circ}\right)$ & $62.7^{\circ}\left( \pm 2.1^{\circ}\right)$ & $47.0^{\circ}\left( \pm 6.2^{\circ}\right)$ & $13.5^{\circ}\left( \pm 8.2^{\circ}\right)$ & 28 \\
\hline $\mathrm{ZYH}$ & $66.0^{\circ}\left( \pm 3.0^{\circ}\right)$ & $65.4^{\circ}\left( \pm 1.6^{\circ}\right)$ & $50.2^{\circ}\left( \pm 2.6^{\circ}\right)$ & $15.8^{\circ}\left( \pm 3.8^{\circ}\right)$ & 7 \\
\hline $\mathrm{PG}$ & $74.9^{\circ}\left( \pm 4.3^{\circ}\right)$ & $46.0^{\circ}\left( \pm 3.6^{\circ}\right)$ & $25.6^{\circ}\left( \pm 4.5^{\circ}\right)$ & $49.3^{\circ}\left( \pm 4.5^{\circ}\right)$ & 7 \\
\hline
\end{tabular}

Static WCA for all five samples agree with the static WCA data in Figure 17 (page 78): static WCA for HOPG and PG is $c a .65^{\circ}$ and $c a .45^{\circ}$, respectively. Moreover, there is no salient difference between HOPG samples. For HOPG, $\mathrm{WCA}_{\mathrm{a}}$ is very near the static WCA indicating that the liquid contact line interacts mostly with the graphitic surface and not with defects; whereas, $\mathrm{WCA}_{\mathrm{r}}$ is lower than static WCA showing the tendency of water to pin at defects. ${ }^{251} \mathrm{PG}$ has substantially different WCA values and dynamic contact angles deviate significantly from the static WCA, a consequence of greater defect density. $\mathrm{WCA}_{\mathrm{a}}$ for $\mathrm{ZYA}$ and $\mathrm{PG}$ is $66.3^{\circ}$ and $74.9^{\circ}$, respectively. Taking error into consideration, the range of $\mathrm{WCA}_{\mathrm{a}}$ is $68.5^{\circ}-64.1^{\circ}$ and $79.2^{\circ}$ $70.6^{\circ}$, respectively. This provides an error range of $15.1^{\circ}-2.1^{\circ}$ for the two samples. Taking the upper limit of $15.1^{\circ}$ still yields an $\mathrm{WCA}_{\mathrm{a}}$ lower than the expected value $\left(90^{\circ}\right)$; however, the high error for PG is due to its lower quality. A more realistic $\mathrm{sp}^{2}$ carbon surface is ZYA. The error of $2.1^{\circ}$ is relatively small compared to the absolute contact angle values and the advancing WCA of high and low quality graphite samples are quite similar. 
Receding contact angles provide very different information, giving insight into the role of defects on the surface. The HOPG samples all have receding contact angles $10-20^{\circ}$ below the static WCA and hysteresis in the same range except for SPI-1 which shows greater hysteresis. Interestingly, the SPI samples appear to have greater hysteresis than the ZYA and ZYH samples. This may be a consequence of their manufacturing processes and provide insight into the sample quality since SPI and Momentive have different fabrication processes (see Section 1.2 on page 2). Moreover, error is generally greater for the SPI samples - especially for SPI-2 - which in addition to AFM may indicate that these samples have more defects than the Momentive samples. However, once experimental error is considered, there is little difference between the four samples and they generally behave similarly. These experiments were not intended to investigate the difference in quality between various HOPG samples and further analysis should be performed before conclusions can be drawn. Nevertheless, there are slight differences between samples of the two manufacturers which can have consequences for their use in research.

Receding WCA of PG is substantially lower than that of HOPG: $c a .25^{\circ}$ and $50^{\circ}$, respectively. This result emphasizes the pinning effect of defects on the receding WCA. PG has many more defects than HOPG (Table 9 on page 63). Water is attracted to the hydrophilic defects, maximizes its interaction with defects, and minimizes its interaction with the hydrophobic graphite. As defect density increases, $\theta_{\mathrm{r}}$ will decrease asymptotically as shown by Raj et al. (Figure 19 on page 85 ). ${ }^{251}$ This behaviour is clearly observed for the graphite sample with higher defect density (PG) when compared to the graphite sample with lower defect density (ZYA). 
The data unequivocally shows a substantial difference between high quality and low quality graphite samples. Hysteresis for ZYA and PG is $10^{\circ}$ and $49^{\circ}$, respectively, and their advancing WCAs are similar. This indicates defects have an effect on the tendency of the surface to retain water (receding WCA) but very little to no influence on its ability to wet (advancing WCA). This is consistent with the findings of Raj et al. (see 5.2.1 on page 80). ${ }^{251}$

Therefore, with significant certainty and using currently available models to explain wettability of chemically heterogeneous surfaces, one can conclude that the experimentally measured advancing WCA on a surface is a measure of its intrinsic wettability. Neither the roughness nor chemical heterogeneity (due to defects) of ZYA and PG are significant enough to affect advancing wettability to a conspicuous degree. This means that the advancing WCA of graphite as shown in Figure 22 and Table 14 is the intrinsic WCA: $\mathrm{WCA}_{\mathrm{a}}$ is $66.3^{\circ}$ and $74.9^{\circ}$ for ZYA and PG, respectively. Considering error, the difference between $\mathrm{WCA}_{\mathrm{a}}$ for the two samples is $2.1^{\circ}$ and the intrinsic WCA of fresh $\mathrm{sp}^{2}$-hybridized carbon is determined to be $70.0^{\circ} \pm$ $1.5^{\circ}$. 


\subsubsection{Calculating surface free energy from hysteresis}

Chibowski et al. presented a method in 2002 which seeks to calculate solid surface energy $\left(\gamma_{\mathrm{s}}\right)$ from advancing and receding contact angle data: ${ }^{245}$

$$
\gamma_{s}=\gamma_{l}\left(\cos \theta_{r}-\cos \theta_{a}\right) \frac{\left(1+\cos \theta_{a}\right)^{2}}{\left(1+\cos \theta_{r}\right)^{2}-\left(1+\cos \theta_{a}\right)^{2}} \quad \text { Equation } 42
$$

Simplifying Equation 42 yields:

$$
\gamma_{s}=\frac{\gamma_{l}\left(1+\cos \theta_{a}\right)^{2}}{2+\cos \theta_{r}+\cos \theta_{a}} \quad \text { Equation } 43
$$

Using the data in Table 14, the surface energy on freshly exfoliated ZYA and PG was calculated to be $48.4 \pm 2.6 \mathrm{~mJ} / \mathrm{m}^{2}$ and $36.6 \pm 6.0 \mathrm{~mJ} / \mathrm{m}^{2}$, respectively. Data for all graphitic samples is shown in Table 15. The calculated surface energy generally correlates inversely to hysteresis, i.e., a small hysteresis results in a higher surface energy and vice versa. Compared to the Neumann, Fowkes, and Owens-Wendt models (Table 6 on page 42) the surface energy calculated using hysteresis is smaller, especially for PG where hysteresis is greatest. Using solidified sulfur samples, Chibowski and Terpilowski observed that the Neumann equation of state model yielded the lowest surface energy which is opposite to the result on HOPG (i.e., Neumann model yields greatest $\gamma_{s}$ ). This may indicate a material dependence although not nearly enough information has been collected on a variety of samples to make any conclusions. $^{177}$ 
Since data in Table 6 was taken on SPI-2, this material provides the fairest comparison of models. Surface energy calculated using hysteresis is analogous to that using the Owens-Wendt model: $50.1 \mathrm{~mJ} / \mathrm{m}^{2}$ and $51.6 \mathrm{~mJ} / \mathrm{m}^{2}$, respectively. This may indicate that the model proposed by Chibowski et al. is similar to the Owens-Wendt model and provide analogous results; however, this must be verified using materials other than graphite. If the models do prove to provide analogous results, they can be used interchangeably depending on user preference: The OwensWendt model uses multiple different liquids and provides dispersive and polar components; whereas, the hysteresis model uses dynamic contact angle of a single liquid and does not provide dispersive and polar components. Chibowski et al. even state that the calculated surface energy will be dependent upon test liquid. ${ }^{245,263}$ Most importantly, Equation 42 was derived using the Young-Dupré equation (Equation 10 on page 30) and film pressure assuming that a liquid film is present behind the receding contact line. This alleviates ambiguity surrounding equation of state and component models (see Section 3.2 on page 27).

Table 15. Surface energy of fresh graphite using various models. Neumann, Fowkes, and Owens-Wendt data was presented in Table 6 (page 42). Data is total surface energy $\left(\gamma_{\mathrm{s}}\right)$ with units $\mathrm{mJ} / \mathrm{m}^{2}$ and is presented as average ( \pm standard deviation).

\begin{tabular}{|c|c|c|c|c|}
\hline & Neumann & Fowkes & Owens-Wendt & $\begin{array}{c}\text { Chibowski } \\
\text { (hysteresis })\end{array}$ \\
\hline ZYA & -- & -- & -- & $48.37( \pm 2.60)$ \\
\hline SPI-1 (ZYA) & -- & -- & -- & $42.66( \pm 1.66)$ \\
\hline SPI-2 (ZYB) & $60.23( \pm 0.86)$ & $55.40( \pm 3.16)$ & $51.60( \pm 0.87)$ & $50.10( \pm 2.68)$ \\
\hline ZYH & -- & -- & -- & $47.30( \pm 3.90)$ \\
\hline PG & -- & -- & -- & $36.60( \pm 5.99)$ \\
\hline
\end{tabular}




\subsection{WENZEL AND CASSIE-BAXTER WETTING MODELS}

In 1936, Robert Wenzel published his work relating contact angle measurements to surface roughness. $^{\S \S}$ His now famous Wenzel equation was the first to quantify the effect of surface roughness on contact angle measurements. Interestingly, this work was performed on waxes, metallic soaps, and gums in an effort to determine how water interacts with surfaces, specifically to better understand the behaviour of waterproofing agents on woven and knitted fabrics. Remarkably, the Wenzel equation has proved to be incredibly robust for predicting wettability of nearly every conceivable solid surface over the past 80 years. ${ }^{172}$

Cassie and Baxter sought to expand upon the work of Wenzel and published their investigation - Wettability of Porous Surfaces - in 1944. ${ }^{182}$ Similar to Wenzel, Cassie and Baxter formulated their theory to explain wetting behaviour of waterproofing agents and improve design of rain resistant fabrics. They also showed that hydrophobicity of duck feathers is due to surface structure, ${ }^{182}$ a concept that has recently become important with the desire to engineer ultrahydrophobic surfaces. Cassie and Baxter studied porous surfaces; whereas, Wenzel studied solid surfaces. This is a critical difference in the application of their equations: the Wenzel equation relates surface roughness to contact angle and the Cassie-Baxter equation relates surface heterogeneity (i.e., chemical heterogeneity) to contact angle.

Using water and hexadecane drops on a well characterized heterogeneous surface, Extrand showed that the apparent contact angle is dependent only on interactions along the

\footnotetext{
$\S \S$ Robert Wenzel was an esteemed research chemist who was originally from Wilkinsburg, a suburb east of Pittsburgh, and earned his doctorate at Stanford University where he also taught before coming back to Pittsburgh. His original work on waterproof materials was performed at the Mellon Institute of Industrial Research which is now part of Carnegie Mellon University and adjacent to the main campus of the University of Pittsburgh. He later was research head at Westinghouse Research in East Pittsburgh. A prominent member of the Pittsburgh Chemists Club and the local ACS chapter, Dr. Wenzel died February 5, 1979 which is nearly 37 years ago. Dr. Wenzel first published his eponymous Wenzel theory in 1936 , exactly 80 years ago. ${ }^{264}$
} 
contact line and not the entire area under the drop. ${ }^{260}$ Figure 24 shows this concept from a view looking down on the drop: the shaded blue area is the surface area underneath the drop and the red line is the perimeter of the drop. Previous to 2003, researchers (aside from Daniel Pease ${ }^{265}$ ) believed that the area under the drop controlled wetting, i.e., the contact angle of a heterogeneous surface is the average of the entire wetted area. Contrarily, Extrand systematically showed that wetting is only a function of interactions at the contact line and independent of the surface underneath the drop. ${ }^{260}$

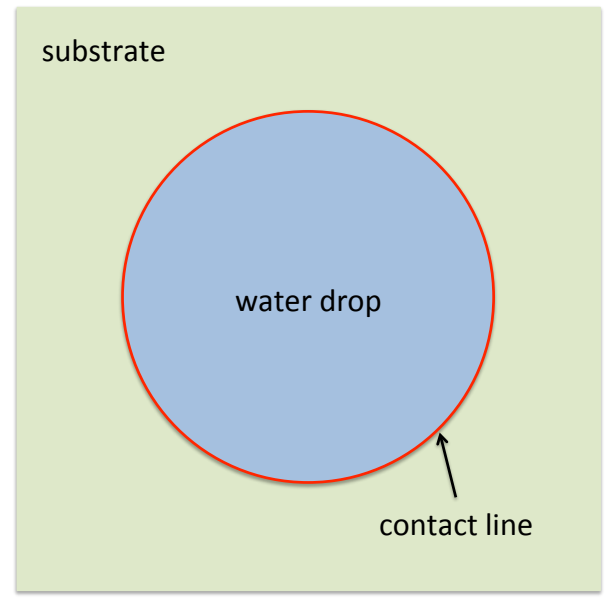

Figure 24. Illustration of water drop on a substrate (overhead view). The contact area is considered to be the entire area between the water drop and substrate (blue region). The contact line is the interface between the substrate, water, and air (red line). 
The Wenzel and Cassie-Baxter models were (mostly) generally accepted as valid until 2007 when Gao and McCarthy published their perspective explaining that the equations are inadequate for describing wetting of real surfaces. ${ }^{261}$ This arose largely because of discrepancies between experimental contact angles and those predicted using the Cassie-Baxter model. Gao and McCarthy used their own data along with already published data of Bartel1 ${ }^{266}$ and Extrand ${ }^{260}$ to make the case that Wenzel and Cassie-Baxter were wrong. ${ }^{261}$

In fact, Daniel Pease published a report in 1949 suggesting that the air-liquid interface is one-dimensional in regards to wetting, meaning that area under the drop has no influence on wettability. ${ }^{265}$ Bartell and Shepard published in 1953 their findings which show inconsistencies between the Wenzel model and real data: drops of water, calcium chloride, and glycerol behave similarly on both smooth paraffin surfaces and paraffin surfaces with roughness within the contact line. ${ }^{266}$ The reports by Pease ${ }^{265}$ and Bartel1 ${ }^{266}$ were generally ignored in relation to their questioning of the Wenzel model. ${ }^{261}$ In 2007, McHale poignantly argued that the Wenzel and Cassie-Baxter equations are valid when used appropriately and Gao and McCarthy misinterpreted assumptions of the original model. Specifically, McHale shows that the equations are valid when the roughness parameter $(r)$ and surface fraction $\left(f_{i}\right)$ are global parameters describing the entire surface and not local parameters describing isolated defects. ${ }^{228}$

Since 2007, there has been substantial debate within literature on the validity of Wenzel and Cassie-Baxter models with researchers debating their limits of applicability and accuracy of assumptions. ${ }^{228,251,253,260-262,267-269}$ Erbil published in 2014 a lengthy review of this debate on how to interpret contact angles on rough and heterogeneous surfaces. ${ }^{250}$ Artificially micropatterned surfaces have provided researchers the ability to systematically investigate the influence of hydrophilic defects on a hydrophobic surface and vice versa..$^{251,253,254}$ See Section 
5.2.1 (page 80) for discussion on how hysteresis has been interpreted using micropatterned surfaces. Several contradictory reports have recently been published on validity of the CassieBaxter equation and various approaches have been suggested for rectifying discrepancies between experimental and predicted contact angles. ${ }^{251,270-275}$ Nevertheless, both Wenzel and Cassie-Baxter models are relatively simple methods for determining the relationship between contact angle and surface roughness/heterogeneity.

Liquid on a solid surface can take two different configurations: (a) complete contact characterized by the Wenzel state and (b) heterogeneous contact characterized by the CassieBaxter state. Figure 25 schematically shows these wetting configurations. ${ }^{276}$ An extreme case of wetting behaviour occurs on superhydrophobic surfaces, in which superhydrophobicity manifests because of multiscale roughness. This situation is commonly modeled by the Cassie-Baxter equation because there is clear heterogeneity in which the (non)wetting liquid interacts with both the solid and trapped air pockets as in Figure 25b-d. Topographical roughness on mechanically exfoliated graphite is not rough enough trap air between surface asperities and the liquid, thus the situation for graphite is more similar to the Wenzel state shown in Figure 25a but with a chemically heterogeneous surface due to defects.

Chemically heterogeneous surfaces are generally believed to have various microscopic contact angles which are a function of the microscopic homogeneous areas. The macroscopic contact angle experimentally observed is an average of the microscopic contact angles, thus the observed behaviour is an average of the microscopic behaviour. The Cassie-Baxter equation remains valid for this wetting state, ${ }^{228,253,260,268,277-279}$ although an alternative has been proposed for atomic scale defects. ${ }^{270}$ 
(a)

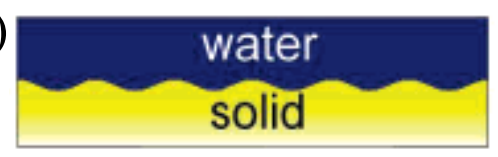

(c)

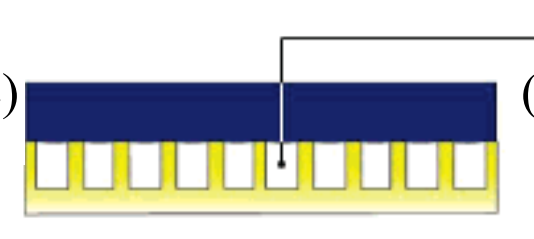

(b)

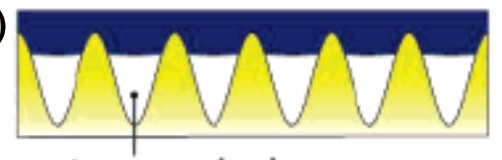

(d) trapped air

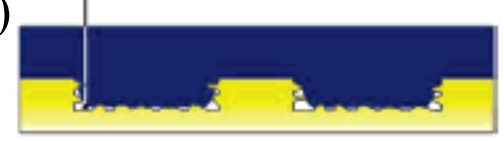

Figure 25. Wetting states of a liquid on a solid surface. Adapted with permission from Zheng, Q. S.; Yu, Y.; Zhao, Z. H. Effects of Hydraulic Pressure on the Stability and Transition of Wetting Modes of Superhydrophobic Surfaces. Langmuir 2005, 21, 12207-12212. Copyright 2005 American Chemical Society. ${ }^{276}$

\subsubsection{Wenzel analysis}

Section 3.6 (page 44) discusses the influence of surface roughness on wettability of graphene. The conclusion was that nanoscale roughness does not affect wettability according to the Wenzel equation (Equation 22 and Equation 23 on page 44). Using the data from $\mathrm{MoS}_{2}$ (Section 6.5 on page 128), ${ }^{70} r$ would have to be 20.5 for the WCA on fresh $\mathrm{MoS}_{2}$ to be $89^{\circ}$ (its generally accepted value). The projected surface area of the image was $2500 \mu \mathrm{m}^{2}$ (50x50 $\mu \mathrm{m}$ AFM scan). To achieve an $r$ value of 20.5 the actual surface area due to roughness would need to be 51,250 $\mu \mathrm{m}^{2}$ or the equivalent of a $226 \times 226 \mu \mathrm{m}$ scan. This is definitely not the case for graphene, graphite, or $\mathrm{MoS}_{2}$ and it is reasonable to conclude that the surface roughness of our $\mathrm{MoS}_{2}$ samples does not influence the observed wettability.

Influence of surface roughness was analyzed for graphite samples according to the Wenzel equation. Young's WCA $\left(\theta_{\mathrm{Y}}\right)$ was calculated to be $65.6^{\circ}$ and $50.9^{\circ}$ for $\mathrm{ZYA}$ and $\mathrm{PG}$, respectively, as shown in Table 16. Roughness has no effect on wettability for any of the HOPG samples and decreases the WCA by $3.5^{\circ}$ for PG. This is consistent with data already reported in 
Reference 70 and Reference 75 . The surface would need to be extremely rough for roughness affect wettability according to the Wenzel equation.

Advancing WCA for ZYA and PG is $66.3^{\circ}$ and $74.9^{\circ}$, respectively (Table 14 on page 93 ). Applying these values to the Wenzel equation, $\theta_{\mathrm{Y}}$ for $\mathrm{ZYA}$ is $66.3^{\circ}$ and $\theta_{\mathrm{Y}}$ for $\mathrm{PG}$ is $75.9^{\circ}$. This indicates that surface roughness has no influence for ZYA and decreases WCA by $1.4 \%$ for PG. Considering experimental uncertainty for water contact angle measurements is $\pm 1^{\circ}$, the effect of surface roughness is negligible. Therefore, Wenzel analysis concludes that surface roughness has a negligible effect on wettability of fresh graphite.

Table 16. Wenzel analysis of graphite samples. Data presented as average ( \pm standard deviation). $R_{a}$ is the surface roughness from AFM scans.

\begin{tabular}{|c|c|c|c|c|}
\hline & ZYA & SPI-1 & SPI- & PG \\
\hline \# of AFM scans & 20 & 11 & 10 & 20 \\
\hline $\mathrm{R}_{\mathrm{a}}(\mathrm{nm})$ & $0.257( \pm 0.103)$ & $1.536( \pm 0.401)$ & $1.181( \pm 0.249)$ & $41.4( \pm 8.0)$ \\
\hline $\begin{array}{c}\text { Projected Surface } \\
\text { Area }(\mu \mathrm{m})\end{array}$ & $25.0( \pm 0.0)$ & $25.0( \pm 0.0)$ & $25.0( \pm 0.0)$ & $26.8( \pm 0.8)$ \\
\hline $\mathrm{r}$ & $1.000( \pm 0.000)$ & $1.000( \pm 0.000)$ & $1.000( \pm 0.000)$ & $1.073( \pm 0.032)$ \\
\hline $\begin{array}{c}\text { Experimental Static } \\
\text { WCA }\left(\theta_{\mathrm{s}}\right)\end{array}$ & $65.6^{\circ}$ & $65.3^{\circ}$ & $65.1^{\circ}$ & $47.4^{\circ}$ \\
\hline \begin{tabular}{c} 
Young's WCA $\left(\theta_{\mathrm{Y}}\right)$ \\
\hline
\end{tabular} & $65.6^{\circ}\left( \pm 0.0^{\circ}\right)$ & $65.3^{\circ}\left( \pm 0.0^{\circ}\right)$ & $65.2^{\circ}\left( \pm 0.0^{\circ}\right)$ & $50.9^{\circ}\left( \pm 1.3^{\circ}\right)$ \\
\hline $\begin{array}{c}\text { Experimental } \\
\text { Advancing WCA }\left(\theta_{\mathrm{a}}\right)\end{array}$ & $66.3^{\circ}$ & -- & -- & $74.9^{\circ}$ \\
\hline \begin{tabular}{c} 
Young's WCA $\left(\theta_{\mathrm{Y}}\right)$ \\
\hline
\end{tabular} & $66.3^{\circ}\left( \pm 0.0^{\circ}\right)$ & -- & -- & $75.9^{\circ}\left( \pm 0.4^{\circ}\right)$ \\
\hline
\end{tabular}




\subsubsection{Cassie-Baxter analysis}

The Cassie-Baxter equation relates chemical heterogeneity of the sample surface to its wettability:

$$
\cos \theta_{C B}=f_{1} \cos \theta_{1}+f_{2} \cos \theta_{2} \quad \text { Equation } 44
$$

where $\theta_{\mathrm{CB}}$ is the Cassie-Baxter contact angle, $\mathrm{f}_{1}$ is the fraction of defects, $\mathrm{f}_{2}$ is the fraction of basal plane, $\theta_{1}$ is the CA corresponding to $f_{1}$, and $\theta_{2}$ is the CA corresponding to $f_{2}$.

Defect density quantitatively reflects the number of point defects in the carbon lattice. A point defect is defined as a vacancy, self-interstitial atom, substitutional impurity atom, or an interstitial impurity atom as shown in Figure 13 (page 53). ${ }^{204}$ The fraction of defects $\left(\mathrm{f}_{1}\right)$ on graphite can be calculated using the defect density calculated from Raman spectra (Table 9 on page 63). Assuming that a point defect is at least the size of a single carbon atom $\left(\mathrm{r}_{\mathrm{d}}\right)$, the area of a single defect can be calculated assuming the defects are circular:

$$
\text { Area per defect }=\pi(73 \mathrm{pm})^{2} \mid \frac{1 \mu m^{2}}{10^{12} \mathrm{pm}^{2}}=1.67 e^{-8} \mu m^{2} \quad \text { Equation } 45
$$

where the radius of the defect is estimated to be equivalent to the covalent radius of a carbon atom $(73 \pm 2 \mathrm{pm}) .{ }^{257,} 258$ The following calculations are solved for ZYA. Total area of all defects on graphite surface can be calculated using the number of defects (Table 10 on page 68) and the area per defect.: 
Total area of defects $=\left(1.67 e^{-8} \mu \mathrm{m}^{2}\right) *\left(1.08 e^{8}\right.$ defects $) \mid \frac{1 \mathrm{~mm}^{2}}{10^{6} \mu \mathrm{m}^{2}}=1.81 e^{-6} \mathrm{~mm}^{2}$

Equation 46

The water drop contacts an area of $4.55 \mathrm{~mm}^{2}$ (assuming axial symmetry; $\mathrm{r}=1.204 \mathrm{~mm}$ from Table 10), thus the area of basal plane is calculated by subtracting the defect area:

Total area of basal plane $=4.55128356 \mathrm{~mm} m^{2}-0.00000181 \mathrm{~mm}^{2}=4.55128174 \mathrm{~mm}^{2}$

Equation 47

Using the preceding equations (Equation 45, Equation 46, and Equation 47) the defect density and basal plane percentage - can be calculated and results are shown in Table 17.

In order to calculate $\theta_{\mathrm{CB}}$ using Equation 44, the fraction of defects and basal plane must be calculated using total area of defects and total area of basal plane in Table 17 (taking $\mathrm{f}_{1}$ to be defects and $\mathrm{f}_{2}$ to be pristine basal surface):

$$
\begin{array}{ll}
f_{1}=\frac{1.81 e^{-6} \mathrm{~mm}^{2}}{1.81 e^{-6} \mathrm{~mm}^{2}+4.55128174 \mathrm{~mm}^{2}}=0.00000040 & \text { Equation } 48 \\
f_{2}=\frac{4.55128174 \mathrm{~mm}^{2}}{1.81 e^{-6} \mathrm{~mm}^{2}+4.55128174 \mathrm{~mm}^{2}}=99.99999960 & \text { Equation } 49
\end{array}
$$

To solve Equation 44, $\theta_{1}$ is taken to be WCA of defects and $\theta_{2}$ is taken to be advancing WCA (WCA $\mathrm{W}_{\mathrm{a}}$ in Table 14 on page 93). Cao et al. reported the WCA on edge defects of graphene to be $10^{\circ} ;^{99}$ therefore, this value was used for $\theta_{1}$ to reflect the defect wettability. In fact, the 
actual choice of $\theta_{1}$ does not change the end result because the defect area $\left(f_{1}\right)$ is extraordinarily small relative to basal area $\left(f_{2}\right)$ and the defect terms are effectively negligible. Now all terms needed to solve Equation 44 have been obtained.

The Cassie-Baxter equation yields $\theta_{\mathrm{CB}}$ of $66.3^{\circ}$ and $74.9^{\circ}$ for ZYA and PG, respectively, which are analogous to the advancing WCA. This result is a consequence of the extremely small amount of point defects relative to basal plane and the data in Table 17 shows that chemical heterogeneity due to defects has no influence on the wettability of fresh graphite (according to the Cassie-Baxter model).

Patel et al. showed that the defect density of ZYA using AFM is $0.3 \%^{50}$ which is negligible for causing a change in $\theta_{\mathrm{CB}}$. Unfortunately, similar data is not obtainable for PG because step edges can not be defined on its bubbly surface. Nevertheless, it is interesting to calculate the number of defects necessary to cause a $5 \%$ change in $\theta_{\mathrm{CB}}$. This entails using Equation 44 and solving for $f_{1}$ to determine the $f_{1}$ value that results in $\theta_{\mathrm{CB}}$ decreasing $5 \%$ from its original value shown in Table 17 (using $r_{d}=1000 \mathrm{pm}$ ). The calculation shows that defect density would need to be $9.0 \%$ and $9.2 \%$ for ZYA and PG, respectively. This means that $100,000 \mathrm{x}$ more defects is required for ZYA to cause a $5 \%$ change in wettability; whereas, $1,000 \mathrm{x}$ more defects is required for PG to cause a 5\% change in wettability. The graphite surface was manually exfoliated before Raman testing so any defects caused by exfoliation would be reflected in the defect density calculated in Table 10. Raman does not detect perfect zig-zag edges and including these type of defects will increase the calculated defect density; ${ }^{200,} 201$ however, it is unlikely that concentration of zig-zag edges will be great enough to cause defect density to increase by 1,000x for either sample. Therefore one can conclude that the intrinsic 
level of defects on the graphite samples is too low by at least a factor of 1000 to significantly influence wettability according to the Cassie-Baxter model.

Table 17. Cassie-Baxter WCA for ZYA and PG. The total area of defects and basal plane is calculated using the defects contacting the water drop in Table 10 and defect radius $\left(\mathrm{r}_{\mathrm{d}}\right)$ of 73 pm. Calculations using $\mathrm{r}_{\mathrm{d}}=1000 \mathrm{pm}$ are shown in Section 5.3.2.1.

\begin{tabular}{|c|c|c|}
\hline & ZYA & PG \\
\hline Drop area $\left(\mathrm{mm}^{2}\right)$ & 4.55128356 & 7.70621705 \\
\hline Total area of defects $\left(\mu \mathrm{m}^{2}\right)$ & 1.81 & 229.64 \\
\hline Total area of basal plane $\left(\mathrm{mm}^{2}\right)$ & 4.55128174 & 7.70598741 \\
\hline$\%$ defects & 0.00004 & 0.00298 \\
\hline$\%$ basal plane & 99.99996 & 99.99702 \\
\hline$f_{1}$ (defects) & 0.00000040 & 0.00002980 \\
\hline $\mathrm{f}_{2}$ (basal plane) & 0.99999960 & 0.99997020 \\
\hline$\theta_{1}\left(\mathrm{WCA}_{\text {defects }}\right)$ & $10.0^{\circ}$ & $10.0^{\circ}$ \\
\hline$\theta_{2}\left(\mathrm{WCA}_{\mathrm{a}}\right)$ & $66.3^{\circ}$ & $74.9^{\circ}$ \\
\hline Cassie-Baxter $\theta$ & $66.3^{\circ}$ & $74.9^{\circ}$ \\
\hline
\end{tabular}

Note: The number of significant digits is to show the magnitude of change because the total area of defects for ZYA and PG is so small. This is not to reflect any particular amount of certainty. The WCA experiment is accurate to $\pm 1^{\circ}$, thus $\theta_{\mathrm{CB}}$ is given to this accuracy. $\theta_{\mathrm{CB}}$ equals the experimental advancing WCA shown in Table 14 (page 93). 
An alternative approach to solving the Cassie-Baxter equation is to substitute the experimental static WCA $\left(\theta_{\mathrm{s}}\right)$ for $\theta_{\mathrm{CB}}$ and solve for $\theta_{2}$ :

$$
\theta_{s}=f_{1} \cos \theta_{1}+f_{2} \cos \theta_{2} \quad \text { Equation } 50
$$

The Cassie-Baxter model is verified to work for graphite if the calculated $\theta_{2}$ is equal to the advancing WCA; however, if $\theta_{2} \neq \theta_{\mathrm{a}}$ then the Cassie-Baxter model fails to accurately represent graphite wetting. Using the $f_{1}$ and $f_{2}$ values in Table 17 where $\theta_{1}=10^{\circ}$ and $\theta_{\mathrm{CB}}=\theta_{\mathrm{s}}, \theta_{2}$ can be solved. The result for ZYA shows that $\theta_{2}$ is similar to $\theta_{\mathrm{a}}$; however, $\theta_{2}=47.5^{\circ}$ for PG yet $\theta_{\mathrm{a}}=$ $74.9^{\circ}$. Because of the relatively low defect density, the Cassie-Baxter model fails to accurately predict the WCA of freshly exfoliated graphite.

This analysis highlights limitations of the Cassie-Baxter model. The model does not account for contact line pinning and assumes that the contact line interacts indiscriminately with defects and the surface. This is shown in Figure 19 (page 85) and Figure 20a (page 89). Real wetting is characterized by contact line pinning which will change the values of $f_{1}$ and $f_{2}$, thus the model does not account for dynamic wetting state which is why the approach to use the Cassie-Baxter model to solve for $\theta_{\mathrm{a}}$ failed (Equation 50). Data on graphite shows that the Cassie-Baxter model does accurately predict static contact angle when defect density is low enough to not have an influence on wettability. Increasing defect density will change $\mathrm{f}_{1}$ and $\mathrm{f}_{2}$ and these changes due to pinning must be accounted for or else the predicted value will increasingly deviate from the experimental value. This is supported by the data of Raj et al. (Figure 19 on page 85$)^{251}$ 


\subsubsection{Comments on Cassie-Baxter analysis}

Several assumptions were made in calculating the defect density and this section seeks to validate the reasoning behind the assumptions.

First, defects are assumed to be circular which is reasonable since carbon atoms are circular and any point defect will take a circular geometry. ${ }^{204}$

Second, the defect radius is assumed to be equivalent to the covalent bonding radius of carbon. Calculations where defect radius $\left(\mathrm{r}_{\mathrm{d}}\right)$ is varied up to $1000 \mathrm{pm}$ show that the defect density is not sensitive to the chosen $r_{d}$ value. Assuming a radius smaller than $73 \mathrm{pm}$ will only decrease the defect density and have no effect on wettability. When $r_{d}$ is increased to $1000 \mathrm{pm}$ the defect density increases from $0.00004 \%$ to $0.00748 \%$ for ZYA and from $0.00298 \%$ to $0.55920 \%$ for PG. Calculating the Cassie-Baxter WCA $\left(\theta_{\mathrm{CB}}\right)$ for the greater defect density yields no change for ZYA and a change of $0.2^{\circ}$ for PG. This means that a surface with $0.5 \%$ defects will only cause a $0.2^{\circ}$ decrease in WCA. Therefore, defect size is not a critical parameter and the calculated $\theta_{\mathrm{CB}}$ essentially remains unaffected.

Third, defects are only point defects. The graphite samples were only mechanically exfoliated and underwent no further processing that would cause artificial defects on the surface. Thus, it is reasonable to assume that only point defects are present on the pristine graphite surface.

Fourth, uniform distribution of defects on the graphite surface is assumed. This is important because reports by Extrand ${ }^{260}$ and Gao and McCarthy ${ }^{261}$ have noted the importance of differentiating between contact area and contact line as shown in Figure 24 (page 99). McHale

pointed out that local defect density should be used (i.e., defect density at the contact line). ${ }^{228,279}$ Contact line can be modeled as an annulus with " $\mathrm{x}$ " thickness; however, defect density within the 
contact area and at the contact line are equivalent when surface defects are uniformly distributed. This means that a particular area on the sample can not be discerned from another area based on its defect density, thus local and global defect density are equivalent and defect density within the contact area equals defect density at the contact line. Assuming uniformly distributed defects on graphite is reasonable since the sample was only mechanically exfoliated and underwent no further processing. Uniform distribution is also important for interpreting Raman data and allows the conclusion to be made that acquired data is a function of the entire sample surface and not dependent on the specific area tested.

\subsubsection{Israelachvili equation}

Jacob Israelachvili and Michelle Gee proposed an alternative form of the Cassie-Baxter equation that should be used when chemical heterogeneity is of atomic (molecular) dimensions: ${ }^{270}$

$$
(1+\cos \theta)^{2}=f_{1}\left(1+\cos \theta_{1}\right)^{2}+f_{2}\left(1+\cos \theta_{2}\right)^{2} \quad \text { Equation } 51
$$

This model is based on averaging the polarizability of surface charges; whereas the CassieBaxter model averages cohesion energies. The atomic scale assumption is reasonable for point defects on graphite and Equation 51 has been used for chemically grafted monolayers. ${ }^{280}$

Applying the Israelachvili equation to the wetting and Raman data on graphite, $\theta$ on PG decreases by $0.5^{\circ}$ when the defect radius is $1 \mathrm{~nm}$ (second row of Table 18), otherwise the data remains unchanged. Fitting the defect density $\left(f_{1}\right)$ to $95 \%$ of $\theta$ (5\% change) requires $c a .9 \%$ of defects on ZYA for both Cassie-Baxter and Israelachvili models; PG requires $c a$. $21 \%$ and $11 \%$ of defects, respectively. Ultimately, the conclusion is that point defects on the graphite surface 
do not affect the intrinsic WCA of ZYA or PG because there are too few defects. 9\% defects would be required on ZYA to cause a substantial change of wetting and either $21 \%$ or $11 \%$ defects - depending on model - would be required on PG. The high quality samples used in experiments has defect density much lower than this as shown by Raman, AFM, and XRD.

Table 18. Cassie-Baxter and Israelachvili wetting model results.

\begin{tabular}{|c|c|c|}
\hline & ZYA & PG \\
\hline$\theta_{\mathrm{CB}} \& \theta_{\mathrm{Is}}{ }^{*}$ & $66.3^{\circ}$ & $74.9^{\circ}$ \\
$\mathrm{f}_{1}$ & $1.8 \mathrm{e}^{-6}$ & $2.3 \mathrm{e}^{-4}$ \\
$\mathrm{f}_{2}$ & 0.99 & 0.99 \\
\hline$\theta_{\mathrm{CB}} \& \theta_{\mathrm{Is}}{ }^{* *}$ & $66.3^{\circ} / 66.3^{\circ}$ & $73.0^{\circ} / 72.5^{\circ}$ \\
$\mathrm{f}_{1}$ & $3.4 \mathrm{e}^{-4}$ & 0.04 \\
$\mathrm{f}_{2}$ & 0.99 & 0.96 \\
\hline $95 \% \theta_{\mathrm{CB}}{ }^{* *}$ & $63.0^{\circ}$ & $65.7^{\circ}$ \\
$\mathrm{f}_{1}$ & 0.09 & 0.21 \\
$\mathrm{f}_{2}$ & 0.91 & 0.79 \\
\hline $95 \% \theta_{\mathrm{Is}}{ }^{* *}$ & $63.0^{\circ}$ & $68.9^{\circ}$ \\
$\mathrm{f}_{1}$ & 0.08 & 0.11 \\
$\mathrm{f}_{2}$ & 0.92 & 0.89 \\
\hline
\end{tabular}

Note: $\mathrm{f}_{1}$ represents defects and $\mathrm{f}_{2}$ represents basal plane. $\theta_{1}=10^{\circ}$ and $\theta_{2}=\theta_{\mathrm{a}} .{ }^{*} \mathrm{r}=73 \mathrm{pm} ; *^{*} \mathrm{r}=1000$ pm. $\theta$ of the first and second row were calculated by keeping $f_{1}$ and $f_{2}$ constant. For the third and fourth row, $\theta$ was constant at $95 \%$ of its respective value and $f_{1}$ and $f_{2}$ were fit to solve the equation. 


\subsubsection{Line energy of the triple phase contact line}

The concept of line energy has been proposed to better describe wetting behavior and quantitatively explain the relationship between advancing, receding, and thermodynamic equilibrium contact angles. The equilibrium contact angle is understood to be the Young's contact angle $\left(\theta_{\mathrm{Y}}\right)$ of a liquid on a perfect surface (i.e., no defects, heterogeneity, or roughness). Real surfaces do not exhibit $\theta_{\mathrm{Y}}$ and the observed contact angle is always a function of intrinsic surface properties, e.g., roughness and heterogeneity. Moreover, real surfaces have an advancing and receding contact angle which describes the tendency of the surface to wet or remain wetted, respectively. Therefore, the observed static contact angle can take any position between $\theta_{a}$ and $\theta_{\mathrm{r}}$ and approaches $\theta_{\mathrm{Y}}$ as the surface becomes more perfect: $\theta_{\mathrm{a}}>\theta_{\mathrm{s}} \approx \theta_{\mathrm{Y}}>\theta_{\mathrm{r}} \cdot{ }^{141,252,281-284} \mathrm{~A}$ mathematical relationship using line energy was proposed by Rafael Tadmor in 2004 to rectify the difference between $\theta_{\mathrm{s}}$ and $\theta_{\mathrm{Y}}$ by relating equilibrium contact angle to dynamic contact angles: 252

$$
\begin{array}{cc}
\theta_{0}=\cos ^{-1}\left(\frac{\Gamma_{A} \cos \theta_{A}+\Gamma_{R} \cos \theta_{R}}{\Gamma_{A}+\Gamma_{R}}\right) & \text { Equation } 52 \\
\Gamma_{A}=\left(\frac{\sin ^{3} \theta_{A}}{2-3 \cos \theta_{A}+\cos ^{3} \theta_{A}}\right)^{1 / 3} & \text { Equation } 53 \\
\Gamma_{R}=\left(\frac{\sin ^{3} \theta_{R}}{2-3 \cos \theta_{R}+\cos ^{3} \theta_{R}}\right)^{1 / 3} & \text { Equation } 54
\end{array}
$$

where $\theta_{0}$ is the thermodynamic equilibrium contact angle which is equivalent to $\theta_{\mathrm{Y}}$. 
The physical explanation of line tension is rather ambiguous and not clearly based on any physical parameters. Equation 52 is mathematically derived using Young's equation (Equation 5 on page 27) by incorporating line tension as an additional term to compensate for surface nonideality. ${ }^{252}$ Line tension is associated with the energy necessary to overcome surface roughness and heterogeneity. Several investigations have sought to further this understanding, ${ }^{281-284}$ however, it is still not clearly understood. Nevertheless, $\theta_{0}$ was calculated using dynamic WCA data (Table 14 on page 93) on fresh graphite.

Table 19 lists the experimental WCA $\left(\theta_{\mathrm{s}}\right)$ and the equilibrium WCA $\left(\theta_{0}\right)$ calculated from Equation 52. There is agreement between $\theta_{\mathrm{s}}$ and $\theta_{0}$ for all of the graphite samples. ZYA and PG have the best agreement with percent difference being $6.7 \%$ and $3.8 \%$, respectively. While the physical phenomena behind the line tension is unclear, the data remain important for showing that the equation proposed by Tadmor does generally reflect the equilibrium contact angle - at least for the graphite surfaces studied - and the experimental static contact angle is similar, but not analogous, to the thermodynamic equilibrium contact angle.

Table 19. Equilibrium WCA using the Tadmor equation.

\begin{tabular}{|c|c|c|c|c|}
\hline & $\begin{array}{l}\text { Advancing } \\
\text { WCA }\left(\theta_{\mathrm{a}}\right)\end{array}$ & $\begin{array}{l}\text { Receding } \\
\text { WCA }\left(\theta_{\mathrm{r}}\right)\end{array}$ & $\begin{array}{c}\text { Static } \\
\text { WCA }\left(\theta_{\mathrm{s}}\right)\end{array}$ & $\begin{array}{c}\text { Equilibrium } \\
\text { WCA }\left(\theta_{0}\right)\end{array}$ \\
\hline ZYA & $66.3^{\circ}\left( \pm 2.2^{\circ}\right)$ & $56.3^{\circ}\left( \pm 2.2^{\circ}\right)$ & $65.7^{\circ}\left( \pm 1.2^{\circ}\right)$ & $61.2^{\circ}\left( \pm 2.2^{\circ}\right)$ \\
\hline SPI-1 (ZYA) & $70.7^{\circ}\left( \pm 1.7^{\circ}\right)$ & $46.2^{\circ}\left( \pm 3.4^{\circ}\right)$ & $66.1^{\circ}\left( \pm 1.9^{\circ}\right)$ & $58.0^{\circ}\left( \pm 2.6^{\circ}\right)$ \\
\hline SPI-2 (ZYB) & $61.7^{\circ}\left( \pm 3.2^{\circ}\right)$ & $47.0^{\circ}\left( \pm 6.2^{\circ}\right)$ & $62.7^{\circ}\left( \pm 2.1^{\circ}\right)$ & $54.2^{\circ}\left( \pm 4.7^{\circ}\right)$ \\
\hline $\mathrm{ZYH}$ & $66.0^{\circ}\left( \pm 3.0^{\circ}\right)$ & $50.2^{\circ}\left( \pm 2.6^{\circ}\right)$ & $65.4^{\circ}\left( \pm 1.6^{\circ}\right)$ & $57.9^{\circ}\left( \pm 2.8^{\circ}\right)$ \\
\hline$P G$ & $74.9^{\circ}\left( \pm 4.3^{\circ}\right)$ & $25.6^{\circ}\left( \pm 4.5^{\circ}\right)$ & $46.0^{\circ}\left( \pm 3.6^{\circ}\right)$ & $49.2^{\circ}\left( \pm 4.0^{\circ}\right)$ \\
\hline
\end{tabular}




\subsection{HOW METHOD OF EXFOLIATION AFFECTS WETTABILITY}

The adhesive tape method is well established for exfoliating lamellar materials such as HOPG. An alternative method of exfoliation is using a razor to physically separate the layers orthogonal to its c-axis. The adhesive tape method was used for all HOPG samples (ZYA, SPI-1, SPI-2, $\mathrm{ZYH})$ used in this investigation; however, PG was not able to be exfoliated using adhesive tape and was exfoliated using a razor.

Uncertainty arose pertaining to the influence of exfoliation method on wettability. This is especially a concern since the WCA of razor exfoliated PG is substantially lower than tape exfoliated HOPG (Figure 17 and Table 11 on page 78). WCA of tape and razor exfoliated ZYA is nearly analogous. WCA for razor exfoliated ZYA aged in air overnight was $92.9^{\circ} \pm 5^{\circ}(\mathrm{N}=5)$. This shows that exfoliation method does not influence WCA on the fresh or aged surface. PG could not be exfoliated with tape, thus no data was taken using the tape method. This data illustrates that exfoliating with razor does not affect wettability and the lower WCA observed for PG is intrinsic to the sample and not a consequence of exfoliation method.

\subsection{CONCLUSIONS}

Freshly exfoliated high and low quality graphite samples were studied in order to elucidate the intrinsic water contact angle of $\mathrm{sp}^{2}$-hybridized carbon. Quantitative analysis of Raman spectra showed that the defect density of ZYA and PG is $24 \pm 16$ and $1778 \pm 1187$ defects $/ \mu \mathrm{m}^{2}$, respectively. Knowing the graphite defect density allowed for the influence of surface defects on wettability to be quantified. Wenzel analysis showed that surface roughness has negligible effect 
on the measured contact angles for all the graphite samples tested. Moreover, Cassie-Baxter analysis showed that chemical heterogeneity caused by high energy defects have little to no effect on the advancing WCA.

Current hysteresis theory says that advancing WCA is attributed to interactions between water and pristine sample surface; whereas, receding WCA is attributed to interactions between water and surface defects. Advancing WCA of ZYA and PG was $66.3^{\circ}$ and $74.9^{\circ}$, respectively. Considering error, the advancing WCA of graphite was determined to be $70.0^{\circ} \pm 1.5^{\circ}$. The receding WCA of ZYA and PG was $56.3^{\circ}$ and $25.6^{\circ}$, respectively. The higher defect density of PG causes the receding WCA to be lower compared to ZYA. Since the advancing WCA can be considered the wettability of $\mathrm{sp}^{2}$-hybridized carbon sans defects, this analysis indicates that fresh (clean) graphite is intrinsically mildly hydrophilic with a WCA of $70^{\circ} \pm 1.5^{\circ}$. 


\subsection{UNDERSTANDING THE INTRINSIC WATER WETTABILITY OF MoS ${ }_{2}{ }^{* * *}$}

\subsection{HISTORY OF 2D MATERIAL WETTABILITY}

Molybdenum disulfide $\left(\mathrm{MoS}_{2}\right)$ has generated significant interest in the past several years as limitations of graphene become apparent due to its zero bandgap. ${ }^{133,285-291}$ Weak interlayer van der Waals forces allow $\mathrm{MoS}_{2}$ to be easily exfoliated to form atomic layers which, like graphene, can be used in electronic and optoelectronic devices for environmental, biological, and clinical applications. ${ }^{285,286,} 289 \mathrm{MoS}_{2}$ and other 2D transition metal dichalcogenides (TMDCs) such as $\mathrm{MoSe}_{2}, \mathrm{WS}_{2}$, and $\mathrm{WSe}_{2}$ are semiconductors that have an intrinsic bandgap which enhances device sensitivity and allows for fabrication of unique field-effect transistors (FETs), biosensors, solar cells, and light-emitting diodes (LEDs). ${ }^{133,287,289,290}$ Additionally, the atomic thinness of TMDCs allow for flexible devices not possible with traditional organic semiconductors. ${ }^{133,287,}$ 288, 291 Sarkar et al. demonstrated that $\mathrm{MoS}_{2}$-based FET biosensors are over 74 times more sensitive than a graphene-based device and can be utilized for ultrasensitive protein sensing at extremely low concentrations of 100 femtomolar. ${ }^{292}$ Moreover, Lee et al. demonstrated efficacy of a $\mathrm{MoS}_{2}$ biosensor for detection of prostate antigens in order to diagnose prostate cancer. The minimum antigen concentration detected by their $\mathrm{MoS}_{2}$-based biosensor was $1 \mathrm{pg} / \mathrm{mL}$ which is

${ }^{* * *}$ Reprinted with permission from Kozbial, A.; Gong, X.; Liu, H.; Li, L. Understanding the Intrinsic Water Wettability of Molybdenum Disulfide $\left(\mathrm{MoS}_{2}\right)$. Langmuir 2015, 31, 8429-8435. ${ }^{70}$ Copyright 2015 American Chemical Society. 
4000 times more sensitive than the current clinical cut-off level. ${ }^{289}$ Jiang et al. created a $\mathrm{MoS}_{2-}$ based FET with a $\mathrm{Hg}^{+2}$ detection limit of $30 \mathrm{pM}$ useful for monitoring anthropogenic mercury in drinking water. ${ }^{293}$ These studies show proof-of-concept that incredible, ultrasensitive devices, which are not possible with atomically thin graphene, can be realized using TMDCs.

Understanding wettability of surfaces is critical for fabricating ultrasensitive devices because small changes in wettability can significantly influence adhesion in heterostructures and impact overall device performance. In 1988, Kelebek reported molybdenite to be hydrophobic with a critical surface tension of $29 \mathrm{~mJ} / \mathrm{m}^{2} .{ }^{294}$ Meanwhile, Zhang et al. reported WCA of sputtered $\mathrm{MoS}_{2}$ as $85^{\circ} .{ }^{295}$ More recent work has corroborated the hydrophobicity of $\mathrm{MoS}_{2}$ : WCA of bulk $\mathrm{MoS}_{2}$ was reported as $88.37^{\circ}$ and $75.8^{\circ} .{ }^{289,296}$ Gaur et al. showed that increasing synthesis temperature of $\mathrm{MoS}_{2}$ thin films from $550^{\circ} \mathrm{C}$ to $900^{\circ} \mathrm{C}$ allowed for controlled diffusion of sulfur atoms through the Mo film to create a well ordered surface with a high degree of crystallinity, resulting in a WCA change from $23.8^{\circ}\left(550^{\circ} \mathrm{C}\right)$ to $91.6^{\circ}\left(900^{\circ} \mathrm{C}\right)$ for $2 \mathrm{D} \mathrm{MoS}_{2}$ films. The low WCA surface was attributed to high energy vertically aligned edge sites due to low synthesis temperature. Moreover, they reported that WCA decreases with number of $\mathrm{MoS}_{2}$ monolayers to approach that of the bulk $\left(88.37^{\circ}\right),{ }^{296}$ behaviour also reported on graphene. ${ }^{237}$ Strano et al. reported $\mathrm{MoS}_{2}$ and other TMDCs to have surface energy of $65-75 \mathrm{~mJ} / \mathrm{m}^{2}$ while Gaur et al. reported surface energy of few layer $\mathrm{MoS}_{2}$ as $44.5 \mathrm{~mJ} / \mathrm{m}^{2}$ (Neumann method) and 40.47 $\mathrm{mJ} / \mathrm{m}^{2}$ (Fowkes method). ${ }^{133,296}$ The discrepancy between surface energy values could be due to different methods used to calculate surface energy along with spontaneous contamination by ambient hydrocarbons. ${ }^{77,}{ }^{297}$ However, $\mathrm{MoS}_{2}$ has been believed to be hydrophobic in all the aforementioned articles with WCA of $76-92^{\circ} .{ }^{133,289,294-296}$ Interestingly, Chow et al. recently reported on the wetting behavior of monolayer and few-layer $\mathrm{MoS}_{2}$ and $\mathrm{WS}_{2}$ supported on silica. 
They showed that WCA of fresh monolayer $\mathrm{WS}_{2}$ increases from $70^{\circ}$ to $83^{\circ}$ upon exposure to ambient air. $^{297}$

Surface contamination is a serious concern for any solid surface, especially atomically thin materials, since contaminants can affect water wettability. A classic example is the adsorption of airborne contaminants onto gold rendering the hydrophilic surface to appear hydrophobic. It took the surface science community more than forty years to conclude that observed hydrophobicity of gold is due to airborne hydrocarbon contaminants and that gold is intrinsically hydrophilic. ${ }^{38-40}$ Graphite has been traditionally believed to be hydrophobic with WCA of $c a .90^{\circ}$; however, recent studies indicate graphite is intrinsically mildly hydrophilic with a WCA of $c a .53-65^{\circ}$ and it adsorbs airborne hydrocarbons in the ambient air to minimize surface energy, i.e., appear more hydrophobic. ${ }^{76,230,241}$ Similar results were also reported for monolayer graphene on copper and multilayer graphene on nickel ${ }^{75,77}$ and the surface energy and wettability of freshly synthesized graphene on copper was found to be dependent upon exposure time to ambient air. ${ }^{75}$ Lai et al. attributed the change in graphene wettability to adsorption of both water molecules and hydrocarbons ${ }^{63}$ and Nioradze et al. demonstrated that HOPG electroactivity is significantly affected by organic impurities in water and air. ${ }^{59,} 62$ Boinovich et al. also showed that hydrocarbon contaminants spontaneously adsorb onto boron nitride nanotubes (BNNTs) and render the BNNTs to be hydrophobic, suggesting that other 2D materials are susceptible to spontaneous hydrocarbon contamination. ${ }^{65}$ Similar results have also been reported in several other studies. ${ }^{49,50,52,55,62,64,239}$ In light of these observations, there is great importance in investigating how airborne contamination affects the wettability of $\mathrm{MoS}_{2}$ due to its salience as a 2D material beyond graphene. 
Herein, we investigate bulk $\mathrm{MoS}_{2}$ to elucidate its intrinsic water wettability, which is likely similar to the wettability of $2 \mathrm{D} \mathrm{MoS}_{2}$. WCA shows temporal evolution with an intrinsic value of $69.0^{\circ} \pm 3.8^{\circ}$ that increases to $89.0^{\circ} \pm 3.1^{\circ}$ after 1 day exposure to ambient air. Surface energy of fresh and aged $\mathrm{MoS}_{2}$ was calculated from contact angle measurements with data indicating that surface energy is a strong function of exposure time to ambient air. ATR-FTIR and ellipsometry indicate that hydrocarbon contaminants adsorb onto freshly exfoliated $\mathrm{MoS}_{2}$, rendering the intrinsically mildly hydrophilic surface hydrophobic. Investigating wettability of bulk $\mathrm{MoS}_{2}$, opposed to monolayer $\mathrm{MoS}_{2}$, provides valuable insight to the true material properties without interfering effects from sample synthesis, processing, and substrate interactions.

\subsection{WATER WETTABILITY AND SURFACE ENERGY}

WCA of freshly exfoliated $\mathrm{MoS}_{2}$ was $69.0^{\circ} \pm 3.8^{\circ}$ tested within 10 seconds of exfoliation and increased to $89.0^{\circ} \pm 3.1^{\circ}$ after 1 day of ambient air exposure as shown in Table 20 . Seven day exposure to ambient air yielded a WCA of $91^{\circ}$ indicating that most, if not all, of the adsorption activity occurred within the first 24 hours; however, diiodomethane contact angle (DCA) increased from $27^{\circ}$ to $44^{\circ}$ between day 1 and day 7 . This dramatic increase of DCA may show dynamic adsorption activity beyond what can be deduced from observing WCA. Since the WCA shows negligible change after day 1 and DCA shows significant change, we hypothesize that a competing adsorption process occurs between smaller (volatile) and larger (less volatile) hydrocarbons present in the ambient environment. WCA does not capture changes in hydrocarbon composition while DCA is sensitive to the chemical nature of adsorbed hydrocarbons. In essence, smaller hydrocarbons initially adsorb leading to the $\sim 90^{\circ}$ WCA then 
larger hydrocarbons displace (or supplement) the hydrocarbons already adsorbed leading to further DCA increase. This hypothesis of dynamic adsorption is supported by ATR-FTIR showing continued increase of methylene stretching peaks between day 1 and day 7 (Figure 26). Advancing and receding WCA follows the same qualitative trend as static WCA further demonstrating the effect of contamination on wettability (see Supporting Info of Reference 70).

Surface energy of fresh and aged $\mathrm{MoS}_{2}$ was calculated by the Fowkes model using contact angles of water and diiodomethane. ${ }^{162}$ Additional details of the calculation procedure can be found in Reference 162. The surface energy of fresh $\mathrm{MoS}_{2}$ was $54.5 \mathrm{~mJ} / \mathrm{m}^{2}$ and decreased to $46.0 \mathrm{~mJ} / \mathrm{m}^{2}$ after 24 hours as shown in Table 20. Moreover, the fresh surface had a significant polar component that accounted for $10 \%$ of the total surface energy of fresh $\mathrm{MoS}_{2}$. Both polar and dispersive components decreased upon exposure to ambient air with the polar component approaching $\leq 1 \mathrm{~mJ} / \mathrm{m}^{2}$. Surface energy continued to decrease approaching $38.6 \mathrm{~mJ} / \mathrm{m}^{2}$ after 7 days exposure to ambient air. The decrease from 46.0 to $38.6 \mathrm{~mJ} / \mathrm{m}^{2}$ between day 1 and day 7 can be attributed to the change in DCA which is a function of the type of adsorbed hydrocarbons. This result signifies that bulk $\mathrm{MoS}_{2}$ has a polar component that contributes to the mildly hydrophilic surface and these polar sites become "shielded" as contaminants adsorb. Moreover, the adsorbed contaminants have surface energy much lower than $\mathrm{MoS}_{2}$, indicated by the overall surface energy decrease. These results indicate that $\mathrm{MoS}_{2}$ surface energy can be controlled by the degree of contamination and potentially by the type of hydrocarbon contaminants adsorbed onto the fresh surface.

The $29 \mathrm{~mJ} / \mathrm{m}^{2}$ surface tension of molybdenite reported by Kelebek is significantly lower than our value. ${ }^{294}$ Moreover, Gaur et al. reported the surface energy of few layer CVD-grown $\mathrm{MoS}_{2}$ as $40.47 \mathrm{~mJ} / \mathrm{m}^{2}$ (Fowkes method) without considering ambient air exposure while our 
value on 7-day aged bulk $\mathrm{MoS}_{2}$ is $38.6 \mathrm{~mJ} / \mathrm{m}^{2}{ }^{296}$ These values are reasonably similar and the discrepancy can be attributed to several factors: (1) fabrication process, (2) aging time, (3) type of contaminants, and (4) material differences between bulk and few-layer $\mathrm{MoS}_{2}$.

Table 20. Contact angle and surface energy calculation results on $\mathrm{MoS}_{2}$ as a function of exposure time in ambient air.

\begin{tabular}{|c|c|c|c|c|c|}
\hline & \multirow{2}{*}{$\begin{array}{c}\text { Water } \\
\text { Contact angle } \\
\text { (WCA) }\end{array}$} & \multirow{2}{*}{$\begin{array}{l}\text { Diiodomethane } \\
\text { Contact angle } \\
\text { (DCA) }\end{array}$} & \multicolumn{3}{|c|}{ Fowkes model } \\
\hline & & & $\begin{array}{c}\gamma_{\mathrm{s}}^{\mathrm{p}} \\
\left(\mathrm{m} / \mathrm{m}^{2}\right)\end{array}$ & $\begin{array}{c}\gamma_{\mathrm{s}}^{\mathrm{d}} \\
\left(\mathrm{mJ} / \mathrm{m}^{2}\right)\end{array}$ & $\begin{array}{c}\gamma_{\mathrm{s}} \\
\left(\mathrm{mJ} / \mathrm{m}^{2}\right)\end{array}$ \\
\hline Fresh & 69.0 & 15.2 & 5.5 & 49.0 & 54.5 \\
\hline $1 \mathrm{~h}$ aged & 72.5 & 19.6 & 3.5 & 47.9 & 51.4 \\
\hline $24 \mathrm{~h}$ aged & 89.0 & 27.1 & 0.6 & 45.4 & 46.0 \\
\hline 7day aged & 90.8 & 44.0 & 1.0 & 37.6 & 38.6 \\
\hline
\end{tabular}

\subsection{SURFACE CONTAMINATION}

ATR-FTIR was performed to elucidate the nature of change on $\mathrm{MoS}_{2}$ surface after ambient exposure. As shown in Figure 26, the first test taken two minutes after exfoliation showed slight methylene stretching indicative of carbon containing compounds. The methylene peaks continue to increase with sustained exposure to ambient air showing significant change up to 24 hours. After one day of exposure, ATR-FTIR shows further growth of methylene peaks, although WCA and ellipsometry (Figure 27) remain constant. This potentially shows rearrangement of 
originally adsorbed hydrocarbons and displacement by larger, less volatile contaminants. WCA and ellipsometry are not sensitive to these changes because the chemistry and thickness of the contaminants remain relatively constant.

Small amounts of hydrocarbons are present on the " 2 min" exfoliated sample which could be explained by (1) initially fast adsorption and (2) the physical structure of $\mathrm{MoS}_{2}$. Surface energy will be greatest on the pristine, freshly exfoliated surface and airborne hydrocarbons will be most attracted to this surface. This adsorption will be extremely fast and initial contamination is likely on the time scale of seconds. ${ }^{76}$ Considering that the ATR-FTIR acquisition time is $2.2 \mathrm{~min}$, there is sufficient time for adsorption of contaminants leading to the small methylene peaks observed in Figure 26.

A second hypothesis assumes that after sample synthesis, hydrocarbon contaminants can adsorb onto the $\mathrm{MoS}_{2}$ surface and slowly intercalate in the van der Waals gaps between sulfur atoms. Hydrogen has been shown to diffuse through $\mathrm{MoS}_{2}$ monolayers under certain circumstances and lithium intercalation is commonly used to exfoliate bulk $\mathrm{MoS}_{2}{ }^{298,}{ }^{299}$ Moreover, intercalation with various alkali and transition metals is being explored as a strategy for tuning and manipulating electronic, optical, and thermal properties of TMDCs. ${ }^{300-302}$ On this basis, it is possible for small aliphatic hydrocarbons to intercalate between sulfur atoms. When the sample is exfoliated, surface contaminants will be removed while sub-surface contaminants will remain intercalated thus being detected by the ATR crystal. The probing depth of our instrument is about $0.50 \mu \mathrm{m}$, which is 3 orders of magnitude larger than the surface hydrocarbon thickness. This will result in detection of adsorbed contaminants on the surface as well as intercalated hydrocarbons. The influence of these sub-surface contaminants on wettability is negligible since intercalation will not affect surface properties; however, this may explain why 
contaminants are detected on the fresh surface by ATR-FTIR. The difference in the adsorption kinetics among contact angle, ellipsometry, and ATR-FTIR experiments can additionally be attributed to different contaminants in the ambient, which results from different lab locations and environmental conditions, as reported previously. ${ }^{76,303}$

Ellipsometry in Figure 27 shows fast adsorption of airborne contaminants within the first $\sim 1$ hour after exfoliation followed by an abrupt slowing of adsorption kinetics. Once a plateau is reached the adsorptive layer is $c a .0 .40 \mathrm{~nm}$ and slowly increases to $c a .0 .50 \mathrm{~nm}$ after 2 hours. ATR-FTIR and ellipsometry results provide experimental evidence that $\mathrm{MoS}_{2}$ is susceptible to contamination by airborne hydrocarbons. As a result, wettability and surface energy concurrently decrease upon exposure to ambient air.

Atmospheric concentration of contaminants is strongly affected by local environmental conditions and seasonal changes between summer and winter months can change concentration by a factor of two. ${ }^{76,101,303,304}$ In practical terms, this means that increasing concentration of contaminants in the environment will concurrently affect both the adsorption rate of contaminants onto a surface and the total thickness of adsorbed contaminants. Our previous work on HOPG alluded to this phenomenon showing that degree of contamination was affected by lab location, i.e., local environmental conditions which influence concentration and type of contaminants in the atmosphere. ${ }^{76}$ Moreover, polycyclic aromatic hydrocarbons (PAHs) have been identified as adsorbing onto graphitic surfaces; ${ }^{101}$ therefore, it is reasonable to believe that $\mathrm{MoS}_{2}$ contamination kinetics suffer consequences similar to graphite.

Considering this, ellipsometry data was taken on four different $\mathrm{MoS}_{2}$ samples at different seasons over two years. Shown in Figure 27a, the data indicates slight changes in both the time scale of adsorption and the maximum film thickness once a plateau is reached. Data obtained in 
October 2013 (samples 1 and 2) generally plateaued by 30 minutes with a total thickness near $0.40 \mathrm{~nm}$, while data obtained in February 2015 (sample 3) plateaued by 40 minutes, although it slowly increased afterwards to a total thickness $c a .0 .50 \mathrm{~nm}$. Interestingly, data taken in April 2015 (sample 4) showed much slower initial adsorption kinetics yet the final thickness remained similar to the other samples. This may indicate that different contaminants were adsorbed in different months; however, local chemistry of the four $\mathrm{MoS}_{2}$ samples may vary significantly (e.g., number of step edges, point defects, etc.) and may additionally contribute to the observed differences. After 24 hours (Figure 27b), the final thickness remains $c a .0 .55 \mathrm{~nm}$ which is similar to HOPG. ${ }^{76}$ The inset of Figure 27b shows the first 2 hours of data taken in April 2015 (sample 4). There are slight differences between the adsorption kinetics and final thickness. This combined with data from Figure 27a indicates that a complex adsorption process occurs on the fresh $\mathrm{MoS}_{2}$ surface that is likely a strong function of local surface chemistry and current ambient conditions (e.g., temperature, pressure, concentration and type of hydrocarbons in air).

These results corroborate several previous reports indicating that higher concentrations of polycyclic aromatic hydrocarbons (PAHs) were detected in air during cold winter months when anthropogenic emissions increased. ${ }^{303-311}$ Indeed, the total contaminant thickness in our experiments was greater during the winter (February) than the fall (October), $0.50 \mathrm{~nm}$ and 0.40 $\mathrm{nm}$, respectively. Interestingly, the time scale of the initially fast adsorption after exfoliation was longer in the winter. This may indicate that different contaminants preferentially adsorb depending on their concentration in the atmosphere, which changes throughout the year. GomezHerrero et al. postulated that PAHs adsorb as islands rather than a homogeneous 2D film onto HOPG. Reasonably, contaminant islands may also form on $\mathrm{MoS}_{2}$ which will additionally influence ellipsometry results because the polarized light will simultaneously interact with 
contaminant islands and bare substrate. ${ }^{101}$ Most significantly, our results conclusively show that freshly exfoliated $\mathrm{MoS}_{2}$ spontaneously adsorbs airborne hydrocarbon contaminants to minimize overall surface energy. These conclusions can be extended to HOPG and other TMDCs as well based on their congruent experimental results. ${ }^{75,76,297}$

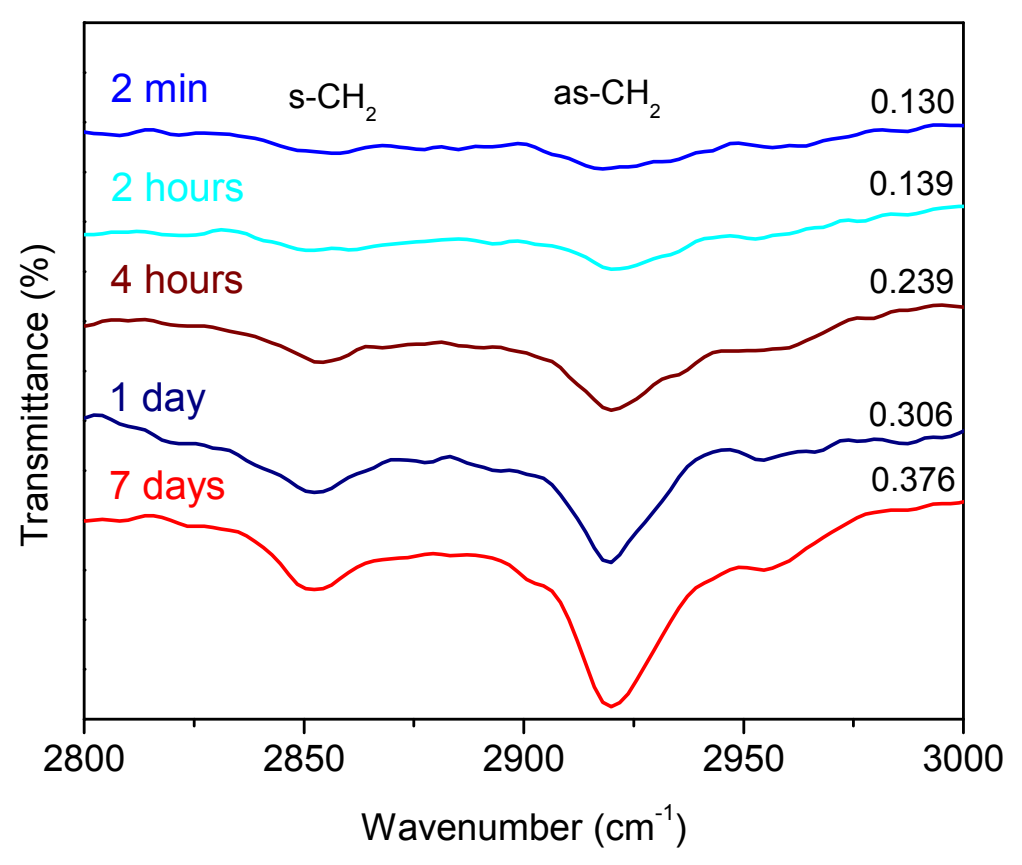

Figure 26. ATR-FTIR of $\mathrm{MoS}_{2}$. Sample was exfoliated and aged in air for specified time. 2 min data takes acquisition time (2.2 minutes) into consideration. Symmetric methylene stretching occurs at $2850 \mathrm{~cm}^{-1}$ and asymmetric methylene stretching occurs at $2920 \mathrm{~cm}^{-1}$. Area of the curve (2830-2980 $\left.\mathrm{cm}^{-1}\right)$ is indicated for each spectrum along the right side of the figure. Data shifted on y-axis for clarity. 
(a)

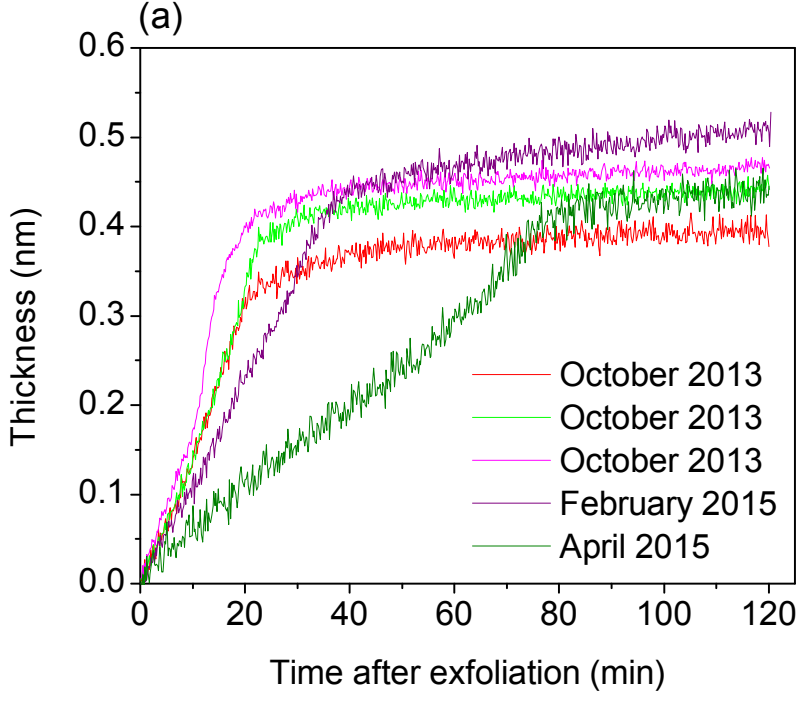

(b)

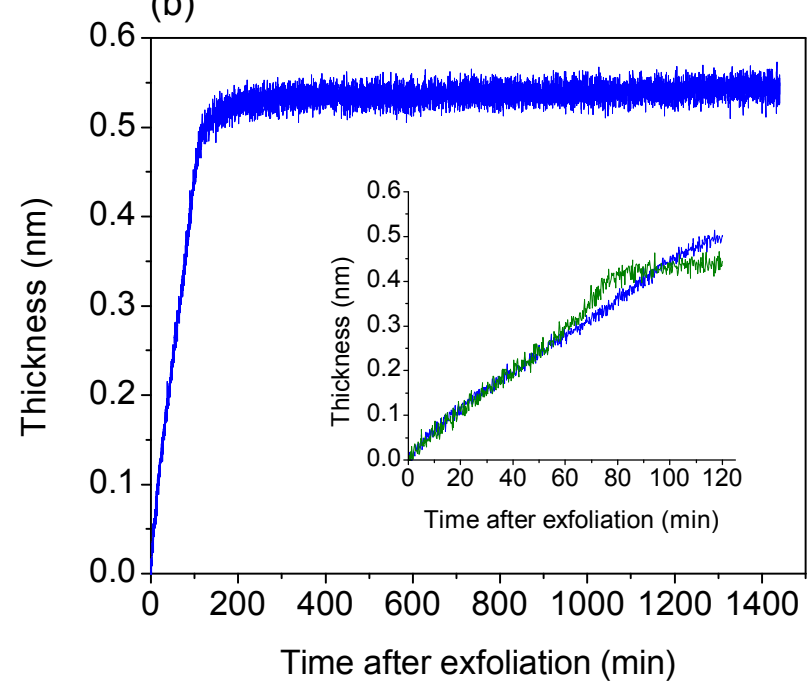

Figure 27. Ellipsometry of freshly exfoliated $\mathrm{MoS}_{2}$. (a) Data collected for 2 hours in October 2013 (samples 1 and 2), February 2015 (sample 3), and April 2015 (sample 4). (b) Data collected for 24 hours in April 2015 (sample 4). Inset: Comparison of first 2 hours of data taken on different days in April 2015 (sample 4) corresponding to same data shown in (a) and (b).

\subsection{CONSEQUENCES OF CONTAMINATION}

Spontaneous contamination of fresh $\mathrm{MoS}_{2}$ results in a film $c a .0 .50 \mathrm{~nm}$ thick that shields the true surface wettability. This can have significant implications for applications that rely on the hydrophobicity of $\mathrm{MoS}_{2}$ as well as provides opportunity for tuning the wettability based on controlled contamination. The data shows that the $0.50 \mathrm{~nm}$ thick contaminant layer concurrently results in a $20^{\circ}$ increase of WCA $\left(69^{\circ}\right.$ to $\left.89^{\circ}\right)$ and an $8.5 \mathrm{~mJ} / \mathrm{m}^{2}$ decrease in surface energy. Figure 28a shows the correlation between WCA and surface energy as a function of surface contamination on $\mathrm{MoS}_{2}$. This relationship can act as a guide for determining extent of contamination of $\mathrm{MoS}_{2}$ as well as understanding how surface energy is affected by contaminants.

Assuming van der Waals interaction between water and substrate, Shih et al. showed through theoretical study that a single graphene layer blocks $70 \%$ of the van der Waals forces 
transmitting from the substrate to the water molecules. ${ }^{237}$ Applying this concept to $\mathrm{MoS}_{2}$ and assuming van der Waals interactions dominate, it is reasonable to conclude that a contaminant layer $0.50 \mathrm{~nm}$ thick will block a significant amount of the attractive forces originating from the substrate (i.e., bulk $\mathrm{MoS}_{2}$ ). Therefore, $30 \%$ would likely be the upper limit for the contribution of forces originating from $\mathrm{MoS}_{2}$ (or HOPG) and experienced by the water molecules assuming that the hydrocarbons have similar translucency to graphene.

Polarized charge distribution is a salient feature of TMDCs that differentiate them from single atom thick van der Waals materials such as graphene that does not have a natural dipole. Exploitation of the charge polarization in $\mathrm{MoS}_{2}$, and other TMDCs, may allow researchers to create next-generation transistors with promising results reported recently. Nguyen et al. showed that $\mathrm{MoS}_{2}$ can be grown preferentially onto ferroelectrically polarized lithium niobate in a process scalable for fabrication of low power transistors. ${ }^{312}$ Moreover, Santos and Kaxiras manipulated the dielectric constant of $\mathrm{MoS}_{2}$ by an external electric field indicating viability for use in uniquely tuned electronic devices. Interestingly, they also propose exfoliation of monolayers using the induced interlayer charge imbalance. ${ }^{313}$ Charge dipoles in self-assembled monolayers impact wettability; ${ }^{314}$ however, the implication for a bulk van der Waals material remains unclear. Reasonably, the observed WCA may be somewhat affected by a surface dipole but its effect may be insignificant for a bulk material; however, an investigation into this topic is necessary. The influence of a surface dipole will subsequently be masked by adsorbed contaminants. 

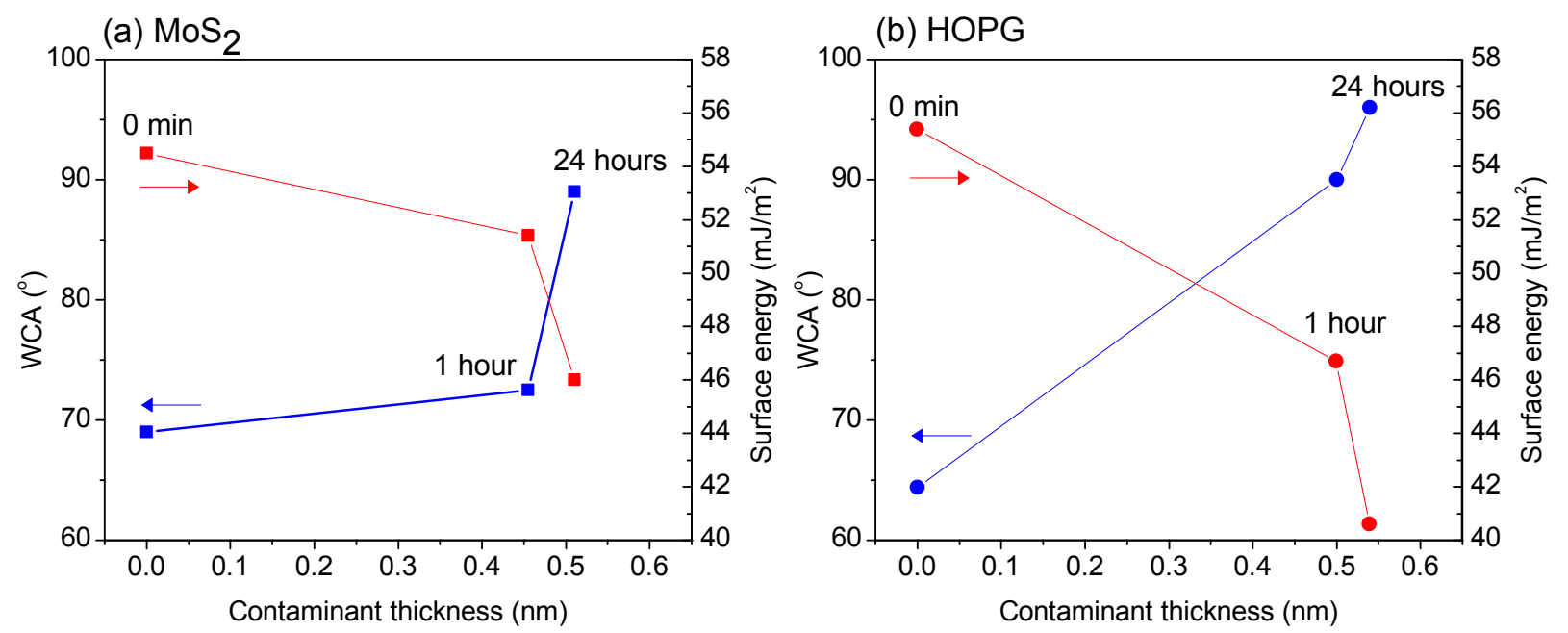

Figure 28. Correlation between surface contamination and WCA/surface energy on (a) $\mathrm{MoS}_{2}$ and (b) HOPG. Solid lines are a visual guide. Data from $\mathrm{MoS}_{2}$ is taken from this study. WCA and contaminant thickness data for HOPG is taken from Reference 76 and surface energy data for HOPG is taken from Reference 75. The leftmost data was taken on fresh surface at 0 min, the center data was taken on surface aged for 1 hour, and the rightmost data was taken on surface aged for 24 hours.

\section{5 $\quad \mathrm{MoS}_{2}$ QUALITY}

Quality of the $\mathrm{MoS}_{2}$ sample was verified by XPS where the sample was exfoliated and immediately placed in the XPS vacuum chamber. Data shown in Figure S1 of Reference 70 indicates that the sample is pure $\mathrm{MoS}_{2}$ and no additional peaks were discerned. ${ }^{315-317}$ Fitting the survey spectrum for Mo $3 \mathrm{~d}$ and S 2p peaks indicated that the results were nearly stoichiometric with atomic percentages of $37.4 \%$ and $62.6 \%$, respectively. The difference from stoichiometry may be due to defects (e.g., point defects, ridges, step edges) on the $\mathrm{MoS}_{2}$ surface and attenuation of sulfur atoms by molybdenum atoms. Additionally, Wenzel analysis shows that surface roughness has a negligible effect on wettability with the Young's contact angle $\left(\theta_{\mathrm{Y}}\right)$ of 
perfectly smooth $\mathrm{MoS}_{2}$ being $69.002^{\circ}$ (see Supporting Info of Reference 70 for calculation details and Figure S2 for AFM images pertaining to Wenzel analysis). ${ }^{172,} 173$

\subsection{COMPARISON OF MOS 2 TO HOPG}

These results on bulk $\mathrm{MoS}_{2}$ should be contrasted to similar studies performed on bulk graphite (Section 2.2 on page 12). Highly ordered pyrolytic graphite (HOPG) has a WCA of $64.4^{\circ} \pm 2.9^{\circ}$ within 10 seconds of exfoliation. Upon exposure to ambient air WCA increases to $c a .90^{\circ}$ after 60 minutes and $97.0^{\circ} \pm 1.8^{\circ}$ after 7 days. ${ }^{76}$ This WCA data on HOPG shows a trend very similar to $\mathrm{MoS}_{2}$ in the current study. Moreover, ATR-FTIR and ellipsometry data provide nearly analogous results. The methylene stretching peaks are very small or negligible on fresh $\mathrm{MoS}_{2}$ and HOPG, respectively, and increase upon exposure time in ambient air. Ellipsometry further indicates temporal dependence upon exposure of a fresh surface to ambient air. The hydrocarbon contaminant layer was $c a .0 .50 \mathrm{~nm}$ on the two surfaces. Similarity between results on HOPG and $\mathrm{MoS}_{2}$ indicate that both materials are intrinsically mildly hydrophilic and adsorb contaminants to reduce surface energy and exhibit their traditionally reported hydrophobicity.

Figure 28 summarizes the consequences of surface contamination on wettability and surface energy for $\mathrm{MoS}_{2}$ and HOPG. ${ }^{75,76}$ Three distinct observations can be made from this data: (1) the overall WCA change of $\mathrm{MoS}_{2}$ is smaller than the WCA change of HOPG, (2) the contaminant layer is thicker on HOPG after 1 hour and the total contaminant thickness for both materials is $c a .0 .55 \mathrm{~nm}$ after 24 hours, and (3) the largest WCA change for $\mathrm{MoS}_{2}$ occurs after 1

hour whereas the largest WCA change for HOPG occurs within the first hour. These observations may provide qualitative insight into the nature of the adsorbed contaminants. 
Assuming the same contaminants are adsorbed onto both $\mathrm{MoS}_{2}$ and $\mathrm{HOPG}$, it can be possible to elucidate qualitative information on the type of contaminants based on their size $(\sim 0.50 \mathrm{~nm})$ and affinity for the fresh substrate (adsorption occurs faster on HOPG).

\subsection{CONCLUSIONS}

Limitations of graphene, namely its zero bandgap, severely limit its usage in many applications ranging from biosensors for cancer detection, ultrasensitive detection of environmental gases, flexible devices, and low-energy LEDs. Fabrication of these devices will require either functionalization of graphene to create a bandgap or other materials. TMDCs have an intrinsic direct and indirect bandgap in their 2D and bulk phases, respectively. This article has provided experimental evidence that bulk $\mathrm{MoS}_{2}$ is intrinsically mildly hydrophilic with a WCA of $69.0^{\circ}$ and surface energy of $54.5 \mathrm{~mJ} / \mathrm{m}^{2}$. Upon exposure to ambient air, the high surface energy adsorbs hydrocarbons from the surrounding environment and becomes contaminated with a $c a$. $0.55 \mathrm{~nm}$ film, thereby reducing apparent surface energy.

Because properties of 2D material-based devices rely strongly on wettability and surface

energy, which are fundamental surface properties that are important for understanding how a particular surface will interact with another surface such as in heterostructures, airborne hydrocarbon contamination is a serious concern that should be taken into consideration during device design. These results potentially extend to all TMDCs - as well as graphene, graphite, black phosphorus, and REOs - and can open new opportunities for these materials when spontaneous hydrocarbon contamination is considered. 


\subsection{RESEARCH DESIGN}

\subsection{SAMPLE PREPARATION}

\subsubsection{HOPG and pyrolytic graphite}

Details of each graphite sample are listed in Table 1 and surface images are shown in Figure 1. Data in Section 2 and Section 3 was collected on SPI-2. Data in Section 4 and Section 5 was collected using primarily ZYA and PG; the sample is appropriately labeled throughout. All samples were used as received with no further cleaning or processing. Exfoliation was performed by the well-established tape method (Scotch brand 1-inch tape) where a piece of adhesive tape (Scotch brand 1-inch) was placed on the sample surface and gently rubbed to ensure contact between the tape and sample. ${ }^{12}$ The tape was then carefully pulled back, removing the upper surface layer, thereby exposing a fresh HOPG surface. Care was taken to ensure that a complete layer of HOPG was removed in each exfoliation. Testing was performed away from HOPG flakes formed by exfoliation. Pyrolytic graphite (PG) was exfoliated by carefully cleaving with a razor blade. This sample was purchased from Graphite Store and used as received. $^{318}$ 


\subsection{2 $\mathrm{MoS}_{2}$}

Bulk $\mathrm{MoS}_{2}$ (2D Semiconductors; $c a .10 \times 5 \times 2 \mathrm{~mm}$ ) was exfoliated with adhesive tape (Scotch brand 1-inch) to expose a fresh surface. ${ }^{12}$ The tape was applied to the upper sample surface and gently pressed to remove air bubbles and ensure contact between the tape and $\mathrm{MoS}_{2}$. The tape was then gently pulled away causing the upper $\mathrm{MoS}_{2}$ layer to be removed, thereby revealing a fresh surface on the bulk sample. The fresh surface was used for experiments only when exfoliation was clean with no flakes and the tape had a uniform coverage of removed material. This ensured that (1) the sample was actually exfoliated exposing a fresh surface and (2) tape residue did not remain on the bulk sample. The fresh $\mathrm{MoS}_{2}$ was tested within 10 seconds to obtain results on the pristine surface. ${ }^{70}$

\subsubsection{Synthesis of CVD graphene}

Four copper foil samples (Alfa Aesar, 99.8\%, $25 \mu \mathrm{m}$ thick) each $\sim 4 \mathrm{~cm}^{2}$ in size were placed at the center of a 1 inch diameter fused quartz tube. The tube was evacuated and heated to $1000{ }^{\circ} \mathrm{C}$ under a $2.0 \mathrm{sccm} \mathrm{H}_{2}$ gas flow at a pressure of 100-110 mTorr for $30 \mathrm{~min}$, followed by $\mathrm{CH}_{4}$ (carbon source) gas flow of $20 \mathrm{sccm}$ at $1000{ }^{\circ} \mathrm{C}$ for another $30 \mathrm{~min}$ at a total pressure of 500 mTorr. Then the copper foil was cooled to room temperature under $\mathrm{H}_{2}$ and $\mathrm{CH}_{4}$ gas flow and taken out from the tube furnace. One $\mathrm{G} / \mathrm{Cu}$ sample was tested immediately and referenced to as "fresh" surface. This sample was exposed to air only during transfer from the CVD chamber to the contact angle instrument ( $\sim 30$ seconds until first contact angle test). The remaining samples were placed in a fume hood to ensure a consistent airborne hydrocarbon contamination level. 
One liquid was used to test the same batch of samples, and testing using different liquids (water, diiodomethane, ethylene glycol, and glycerol) was conducted on different batches of samples. ${ }^{75}$

\subsection{WATER CONTACT ANGLE (WCA)}

Contact angle measurements were taken using a VCA Optima XE goniometer (AST Products) in ambient air at $22-25^{\circ} \mathrm{C}$ and $20-40 \%$ relative humidity with deionized (DI) water supplied from a Millipore Academic A10 with total organic carbon below $40 \mathrm{ppb}$. Contact angles were recorded by a charge coupled device (CCD) camera and analysis was performed using vendor supplied software. Static contact angles were conducted using $2 \mu \mathrm{L}$ water drops, unless otherwise noted. CAs of the fresh surface were taken within 10 seconds of exfoliation. All drops were placed on sample surface not previously exposed to water, thereby alleviating any influence from wetting history. $90^{\circ}$ is generally considered the critical value between hydrophilic $\left(\mathrm{CA}<90^{\circ}\right)$ and hydrophobic $\left(\mathrm{CA}>90^{\circ}\right)$ behavior. Here we define $\mathrm{CAs}$ close to $90^{\circ}$ are "hydrophobic" and lower CAs are more hydrophilic. Diiodomethane (99\%), ethylene glycol (99.8\%), and glycerol (99\%) were purchased from Sigma-Aldrich and used as received. ${ }^{75,76}$

\subsubsection{Graphene}

A separate testing syringe was dedicated for each test liquid to avoid cross-contamination. A liquid drop of $2 \mu \mathrm{L}$ was formed at the end of the syringe and carefully deposited onto the sample surface. The syringe was withdrawn and the image of static contact angle was taken within 3 seconds. The reported contact angle values are based on 5-8 repeats. ${ }^{75}$ 


\subsection{2 $\mathrm{MoS}_{2}$}

Static contact angles were conducted using $2 \mu \mathrm{L}$ water drops and $1 \mu \mathrm{L}$ diiodomethane drops. ${ }^{70}$

\subsubsection{Saturated 1-octadecene vapor bath for HOPG}

A saturated 1-octadecene environment was created by thoroughly washing a glass petri dish with acetone and stacking glass microscope slides in the middle to create a plateau to set the HOPG sample without coming into direct contact with the liquid hydrocarbon. Then $\sim 10 \mathrm{~mL}$ of 1octadecene was added to create a reservoir. The petri dish lid was put in place to create a closed environment where HOPG can be exposed to 1-octadecene at a greater partial pressure than in ambient air. The HOPG was removed from the closed petri dish for testing at the indicated time and replaced back into the closed environment immediately after testing. Aside from transfer and testing time, about 20 seconds total, the sample remained within the closed petri dish. ${ }^{76}$

\subsection{ATR-FTIR}

Measurements were conducted using a Bruker VERTEX-70LS FTIR and a Bruker Hyperion 2000 FTIR microscope in reflectance mode. A germanium 20x ATR objective was used along with a MCT A detector $\left(7000-600 \mathrm{~cm}^{-1}\right)$ cooled by liquid nitrogen. The system was purged for 20 minutes with nitrogen gas before the instruments were powered "ON." This purging allowed for removal of water and ambient gases within the instrument tubing; however, residual water and gases still remained because the system was only purged and not under vacuum. Peaks of 
water and $\mathrm{CO}_{2}$ are outside the wavenumber region of interest in this study and do not affect results. The nitrogen gas purge flowed throughout all experimentation and was only turned off at instrument shutdown. The instruments were powered "ON" and the liquid nitrogen reservoirs filled until the detectors were appropriately cooled. A background scan was taken of the surrounding air with the ATR objective set to pressure setting 1; the germanium crystal was not in contact with the sample during the background scan. The germanium ATR crystal was contacted with the HOPG sample at pressure setting 1 and spectra were collected for 150 scans at $4 \mathrm{~cm}^{-1}$ resolution with an acquisition time of 2.2 minutes. Vendor supplied software was used to analyze spectra with corrections applied for atmospheric $\mathrm{H}_{2} \mathrm{O}$ and $\mathrm{CO}_{2}$. HOPG was exfoliated using the tape method and the first test after exfoliation was taken within 10 seconds. The instrument was located in an analytical characterization laboratory. ${ }^{70,76}$

\subsection{SPECTROSCOPIC ELLIPSOMETRY}

Measurements were conducted with an Alpha-SE (J.A. Woollam Co.) spectroscopic ellipsometer with a wavelength range of $380 \mathrm{~nm}$ to $900 \mathrm{~nm}$ and an incident angle of $70^{\circ}$. Data was collected in situ with an acquisition time of 10 seconds at 10 second intervals. Modeling and analysis of data was performed using vendor-supplied software, CompleteEASE. The instrument was located in a wet chemistry laboratory. The effective optical constants of HOPG and $\mathrm{MoS}_{2}$ were determined using the b-spline model. ${ }^{70,76}$ See Appendix A for details pertaining to the ellipsometry measurements. 


\subsubsection{Graphene}

Ellipsometry measures the change in $\Psi$ and $\Delta$ of polarized light after interacting with a surface. Here $\Psi$ represents the shift in amplitude and $\Delta$ represents the shift in phase of the polarized light. ${ }^{105,180,319,320}$ Previous studies have showed that the change in phase shift $(\delta \Delta)$ is proportional to the film thickness. ${ }^{180,} 181$ The organic contamination on $\mathrm{SiO}_{2}$ has been characterized with ellipsometry and an increase of $\Delta$ with exposure time to organic contaminants was reported. ${ }^{105}$ In the current study, ellipsometry characterization was conducted on $\mathrm{G} / \mathrm{Cu}$ samples with respect to the aging time in ambient air to monitor the possible airborne hydrocarbon contamination. Since $\Delta$ is most sensitive to thickness changes at low wavelength, ${ }^{321}$ we monitored $\Delta$ at $501 \mathrm{~nm}$ and compared initial $\Delta$ on fresh $\mathrm{G} / \mathrm{Cu}$ to the $\Delta$ value obtained on the aged surface. The difference $\left[\Delta_{\text {fresh }}-\Delta_{\text {aged }}(\text { time })\right]_{\text {wavelength }}=501 \mathrm{~nm}$ was taken as $\delta \Delta$ and plotted against the time exposed to ambient air. $^{75}$

\subsection{RAMAN SPECTROSCOPY}

Micro-Raman spectra were obtained on a custom built setup using a $532 \mathrm{~nm}$ solid-state laser. The spot size was less than $\sim 1 \mu \mathrm{m}$ with laser power below $2 \mathrm{~mW}(\mathrm{HOPG})^{76}$ or $1 \mathrm{~mW}$ (graphene $)^{75}$ to avoid any thermal damage to the sample surface. A detailed description of this micro-Raman setup can be found in previous literature. ${ }^{77,} 108,322$ The integration time was 30 seconds (HOPG) or 60 seconds (graphene) and the spectra have been shifted along the y-axis for clarity. ${ }^{75,76}$ 


\subsection{X-RAY PHOTOELECTRON SPECTROSCOPY (XPS)}

Chemical analysis will be performed using a Thermo Scientific ESCALAB 250Xi X-ray Photoelectron Spectrometer (XPS) Microprobe with monochromated Al Ka x-ray source and $200 \mu \mathrm{m}$ spot size. Charge compensation is supplied by a low-energy electron source. The sample is inserted into the analysis chamber and the centre of rotation calibrated for angleresolved (AR) testing. Then the charge compensation flood gun and x-ray gun are turned on, respectively, and a survey spectrum collected using 10 scans and a pass energy of $150 \mathrm{eV}$. Next a high-resolution C1s scan is collected from 279-298 binding energy (eV) using 10 scans and a pass energy of $50 \mathrm{eV}$. Spectra analysis is performed using Thermo Avantage software. Data is collected at $0^{\circ}$ where the photoelectron emission and the surface normal are equal and at $60^{\circ}$ to increase surface sensitivity.

\subsection{ATOMIC FORCE MICROSCOPY (AFM)}

AFM imaging was performed in tapping mode on a Veeco Dimension V instrument using NSC15 Al-backside (MikroMasch; 40 N/m; 325 kHz) and NSC14 Al-backside (MikroMasch; $5.0 \mathrm{~N} / \mathrm{m} ; 160 \mathrm{kHz}$ ) cantilever probes. 


\subsection{X-RAY DIFFRACTION (XRD)}

XRD experiments will be conducted with a high-sensitivity modular x-ray diffraction system (D8 DISCOVER with GADDS) with a Cu x-ray source.

\subsection{ULTRA HIGH VACUUM (UHV) TREATMENT OF HOPG}

UHV experiments were conducted at a base pressure less than $1 \times 10^{-9}$ torr. The HOPG sample was exfoliated and aged in ambient air for 2 weeks. The sample was then placed in the vacuum chamber of a custom built XPS instrument and was evacuated to the appropriate pressure for 15 hours. ${ }^{76}$

\subsection{ULTRAVIOLET/OZONE (UV/O $\left.{ }_{3}\right)$}

$\mathrm{UV} / \mathrm{O}_{3}$ treatment was performed with a Bioforce Nanosciences UV/Ozone Procleaner. The HOPG sample was placed on the borosilicate glass platform inside the loading tray and treated for 5 minutes. ${ }^{76}$ 


\section{APPENDIX A}

\section{DETAILS OF ELLIPSOMETRY DATA ANALYSIS ${ }^{\dagger \dagger \dagger}$}

Ellipsometry measures the polarization state change of light upon reflection from a sample and has been widely used in real time to monitor film growth with data collected within seconds. ${ }^{319,}$ ${ }^{320}$ In this study, we monitored hydrocarbon adsorption onto freshly exfoliated HOPG. The high sensitivity of ellipsometry to sub-nanometer $(\mathrm{nm})$ film is crucial to observe the temporal surface change. Two measured quantities, $\Psi$ and $\Delta$, represent the ratio of Fresnel reflection coefficients between parallel (p) and perpendicular (s) polarized light. $\Delta$, which reflects phase differences of the p- and s-polarized light, is very sensitive to small surface changes. Measured values of $\Psi$ and $\Delta$ are shown in Figure 29 at specific wavelengths. As expected, $\Delta$ is more sensitive to surface changes and sensitivity is greater at lower wavelengths for both $\Psi$ and $\Delta .{ }^{321}$

An optical model is necessary to determine material dielectric functions $\left(\varepsilon=\varepsilon_{1}-i \varepsilon_{2}\right)$ and film thickness. The dielectric functions of the HOPG substrate were extracted from the first data point, considered uncontaminated HOPG, after exfoliation using a Kramers-Kronig consistent bspline layer. ${ }^{323}$ Optical constants of HOPG $\sim 10$ seconds after exfoliation are shown in Table 21

†† Published previously in the Supplementary data of Kozbial, A.; Li, Z.; Sun, J.; Gong, X.; Zhou, F.; Wang, Y.; Xu, H.; Liu, H.; Li, L. Understanding the Intrinsic Water Wettability of Graphite. Carbon 2014, 74, 218$225 .^{76}$ 
and Figure 30a. $\mathrm{n}$ is the index of refraction and $\mathrm{k}$ is the extinction coefficient related by the complex refractive index, $\mathrm{N}=\mathrm{n}-\mathrm{ik}$, which is related to the complex dielectric function $(\varepsilon)$. At $1.996 \mathrm{eV}$, our measured $\mathrm{n}$ and $\mathrm{k}$ are $3.202 \pm 0.030$ and $1.836 \pm 0.027$, respectively, matching well with literature values. ${ }^{324}$ Surface adsorption of hydrocarbons does not affect the optical constants of HOPG (Table 21). The HOPG optical constants were then fixed during modeling of the adsorbed hydrocarbon layer.

Simultaneous measurement of thickness and optical constants are difficult using ellipsometry; ${ }^{325}$ therefore, the adsorbed hydrocarbon layer on HOPG was modeled using a Cauchy dispersion equation with fixed index of refraction:

$$
\mathrm{n}(\lambda)=\mathrm{A}+\frac{\mathrm{B}}{\lambda^{2}}=1.45+\frac{0.01}{\lambda^{2}} \quad \text { Equation } 55
$$

where $\mathrm{n}$ is the refractive index and $\lambda$ is the wavelength in microns. The Cauchy model is appropriate for transparent and dielectric thin films and assumes no absorption of light (i.e., $\mathrm{k}=0),{ }^{321,325}$ correlating well to the thinly adsorbed hydrocarbon film on HOPG. Selection of the "A" constant in Equation 55 depends on the nature of material being adsorbed. For this study, $\mathrm{A}=1.45$ is an appropriate estimate for airborne hydrocarbon contamination of sub-nm thickness. ${ }^{326,} 327$ The effect of varying "A" is shown in Figure 30b. The "one-film" model approach is well documented and applicable to systems with a constant substrate (e.g., HOPG) and a film over-layer (e.g., hydrocarbon). ${ }^{324}$ See Figure 30c for the a schematic of the "one-film" model. 
Table 21. Index of refraction (n) and extinction coefficient (k) of SPI-2 HOPG. Data collected at $1.996 \mathrm{eV}$. Optical constants are determined from the b-spline model. Data presented as the average (standard deviation) of 11 experiments. Exfoliated data taken $\sim 10$ seconds after exfoliation of upper HOPG surface. Aged data taken $\sim 60 \mathrm{~min}$ after exfoliation.

\begin{tabular}{|c|c|c|}
\hline & $\mathrm{n}$ & $\mathrm{k}$ \\
\hline Exfoliated & $3.202(0.030)$ & $1.836(0.027)$ \\
\hline Aged & $3.169(0.029)$ & $1.852(0.031)$ \\
\hline
\end{tabular}
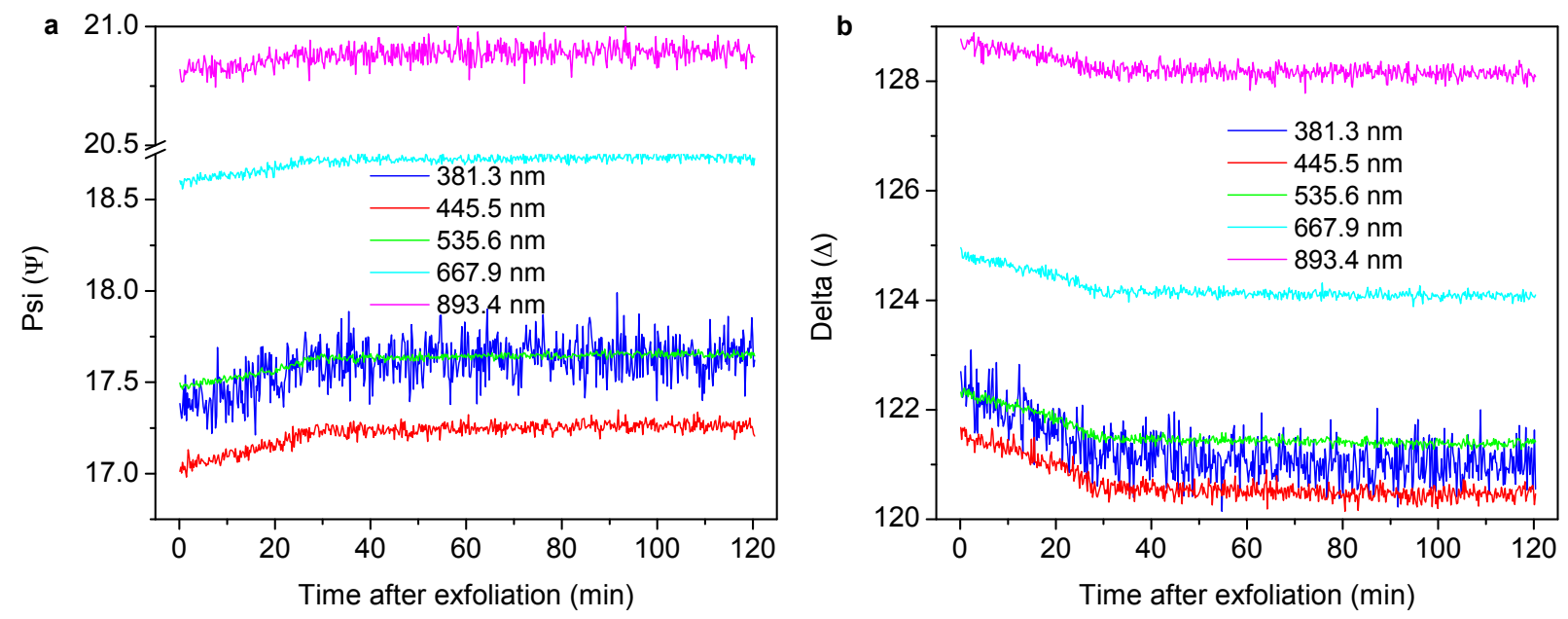

Figure 29. Temporal monitoring of ellipsometric quantities. (a) Psi ( $\Psi$ ) and (b) Delta $(\Delta)$ after HOPG exfoliation. 

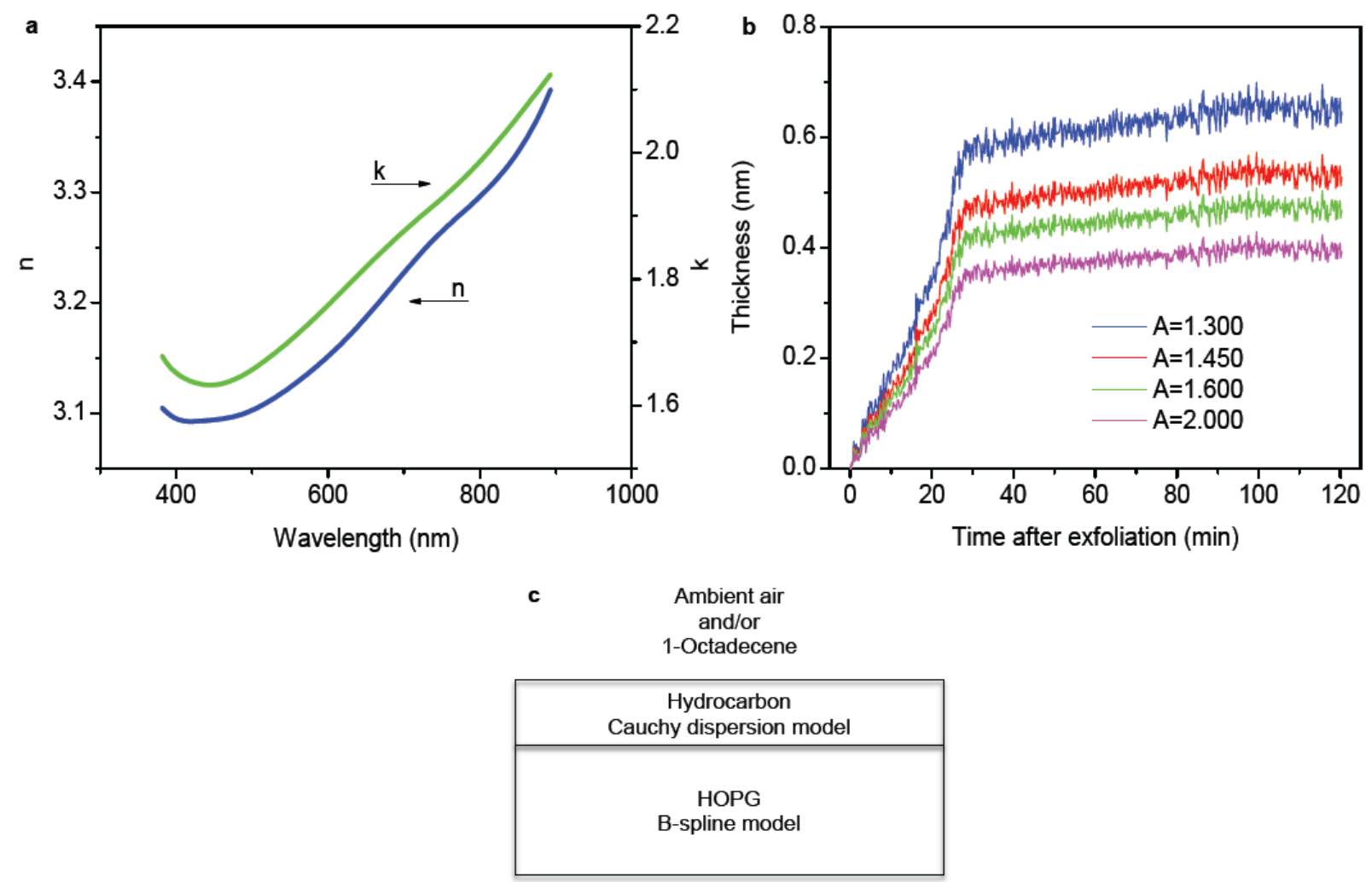

Figure 30. Details of ellipsometry data for HOPG. (a) Index of refraction (n) and extinction coefficient (k) of exfoliated HOPG 10 seconds after exfoliation. (b) Effect of varying value of the "A" constant in Cauchy model for determining thickness of adsorbed hydrocarbon layer on exfoliated HOPG. $A=1.450$ is an appropriate estimate for the refractive index of hydrocarbon mixtures. $^{326,327}$ (c) "One-film" model used for ellipsometric measurement of adsorbed hydrocarbon film thickness. 


\section{APPENDIX B}

\section{ELUCIDATING THE CRYSTAL STRUCTURE OF GRAPHITE}

X-ray diffraction (XRD) is an analytical technique for determining the crystallographic structure

of materials. $^{328}$ The layers of graphite are stacked in an organized ABAB manner and show only a single rocking curve at $c a .26 .52 \theta$ which originates from the (002) plane. ${ }^{11,329} \mathrm{Cu} \mathrm{K \alpha}$ radiation is generated at wavelengths of $1.541 \AA(\mathrm{K} \alpha 1)$ and $1.544 \AA(\mathrm{K} \alpha 2)$, thus a auxiliary peak at slightly higher $2 \theta$, due to the $\mathrm{K} \alpha 2$ radiation, must be stripped from the spectrum (the small peaks toward higher $2 \theta$ are artifacts of the $K \alpha 2$ stripping). The only other peaks observed in the spectra were satellite peaks of the (002) rocking curve which are shown in Figure 31c. Data was collected using a Bruker D8 DISCOVER x-ray diffractometer.

Pristine HOPG will have a single, well defined peak around $26.5^{\circ}$; however, Figure 31 shows two peaks resolved for all the HOPG samples (after K $\alpha 2$ stripping). This indicates that either (a) the sample has two different crystal structures, (b) there is a difference of interlayer spacing $-\mathrm{d}-$ throughout the sample, or (c) the two peaks are an artifact of K $\alpha 2$ stripping. The second and third case is most reasonable considering that both peaks are very near the expected $2 \theta$ for graphite. XRD data on these samples were collected after being exfoliated numerous times for other experiments, thus slight damage to the sample may have occurred (see Section 
4.2.2 for further analysis on surface damage caused by exfoliation). Moreover, Raman spectroscopy shows that the HOPG samples have interlayer coupling of the graphene layers (ABAB stacking) indicated by resolution of two 2D peaks (Figure 14). ${ }^{208}$ Nevertheless, the data unequivocally shows a strong (002) rocking curve and the calculated d value is reasonable (Table 22).

Spectra of the different HOPG samples are nearly analogous and consistent with previous reports (Figure 31; peak fitting parameters are shown in Table 22). ${ }^{2,} 7,11,329$ The salient difference between samples is that PG shows a broad peak at $26.0^{\circ} 2 \theta$ with a FWHM much larger than HOPG. The peak position for PG is shifted $c a .0 .50^{\circ}$ towards lower diffraction angle (20) which is a consequence of increased disorder within the sample. Li et al. showed through simulations that rotation, translation, curvature, and changes in interlayer spacing of the graphene layers cause the diffraction angle to decrease and the FWHM to increase. XRD, however, does not provide decoupled information on the type of disorientation that causes changes in rocking curve lineshape; therefore, further information can not be deduced. ${ }^{11}$

$\mathrm{XRD}$ is not a surface sensitive technique and provides information from the bulk material only; therefore, imperfections on the sample surface may be washed out because their contribution to the overall spectrum is very small. The x-ray penetration depth for graphite was calculated using an Excel routine provided for free download by the Industrial Group of the British Crystallographic Association (xrdpenetr). ${ }^{330}$ The calculated x-ray penetration depth to attain $99 \%$ maximum intensity was $508 \mu \mathrm{m}$ (762 $\mu \mathrm{m}$ to attain $99.9 \%$ maximum intensity; $2 \theta=26.5 ; \rho=2.26 \mathrm{~g} / \mathrm{cm}^{2}$ ). Considering the thickness of a single graphene layer is $3.35 \AA$, the $\mathrm{x}-$ ray sampling depth was $>1.5$ million graphene layers. ${ }^{331}$ 
Professor William Henry Bragg and his son, William Lawrence Bragg, won the Nobel prize in 1915 for determining the crystal structures of $\mathrm{NaCl}, \mathrm{ZnS}$, and diamond. ${ }^{332}$ This work was made possible by their observations that the constructive interference of x-rays occurs at phase shift multiples of $2 \pi$ and is strongest when $\theta$ satisfies the Bragg equation:

$$
2 \mathrm{~d} \sin \theta=\mathrm{n} \lambda \quad \text { Equation } 56
$$

where $\mathrm{d}$ is the interlayer spacing between atoms, $\theta$ is the scattering angle, $\mathrm{n}$ is a positive integer (which will be taken as 1 ), and $\lambda$ is the wavelength of incident $\mathrm{X}$-rays. ${ }^{333,334}$

Applying the Bragg equation to the XRD data (Table 22), the interlayer spacing for ZYA and PG is $3.36 \AA$ and $3.43 \AA$, respectively. The calculated $\mathrm{d}$ value for the HOPG samples, perfectly match the expected value for graphite..$^{9,11,331,335}$ The $\mathrm{d}$ value for PG is slightly larger because the sample is not highly oriented. ${ }^{11}$ The XRD data provides evidence that the HOPG and PG samples are $\mathrm{sp}^{2}$-hybridized carbon since the only response is from the (002) plane. ${ }^{11}$ Furthermore, there are no detectable differences between the HOPG samples and PG can be classified as turbostratic carbon which corroborates Raman results (Section 4.1.1 on page 54). 
Table 22. XRD fitting parameters and interlayer spacing. Data fit using the PsdVoigtl function where $\mathrm{x}_{\mathrm{c}}$ was held constant and A, FWHM, and $\mu$ were allowed to fit (see Table 27 and Table 28 in Appendix D). Fitting data is for the leftmost (green dashed line) data. The calculated interlayer spacing (d) is calculated from the Bragg equation.

\begin{tabular}{|c|c|c|c|c|}
\hline & ZYA & SPI-2 & ZYH & PG \\
\hline $\mathrm{x}_{\mathrm{c}}(2 \theta)$ & 26.48 & 26.45 & 26.44 & 25.99 \\
\hline $\mathrm{A}$ & 8.88 & 19.09 & 20.61 & 59.61 \\
\hline FWHM & 0.058 & 0.091 & 0.085 & 0.426 \\
\hline$\mu$ & 1.04 & 1.57 & 1.48 & 0.43 \\
\hline $\mathrm{d}(\AA)$ & 3.36 & 3.37 & 3.37 & 3.43 \\
\hline \multicolumn{5}{|l}{} \\
\hline $\mathrm{R}^{2}$ & 0.95 & 0.92 & 0.90 & 0.99 \\
\hline $\mathrm{X}^{2}$ & 8.11 & 11.57 & 23.44 & 2.32 \\
\hline
\end{tabular}



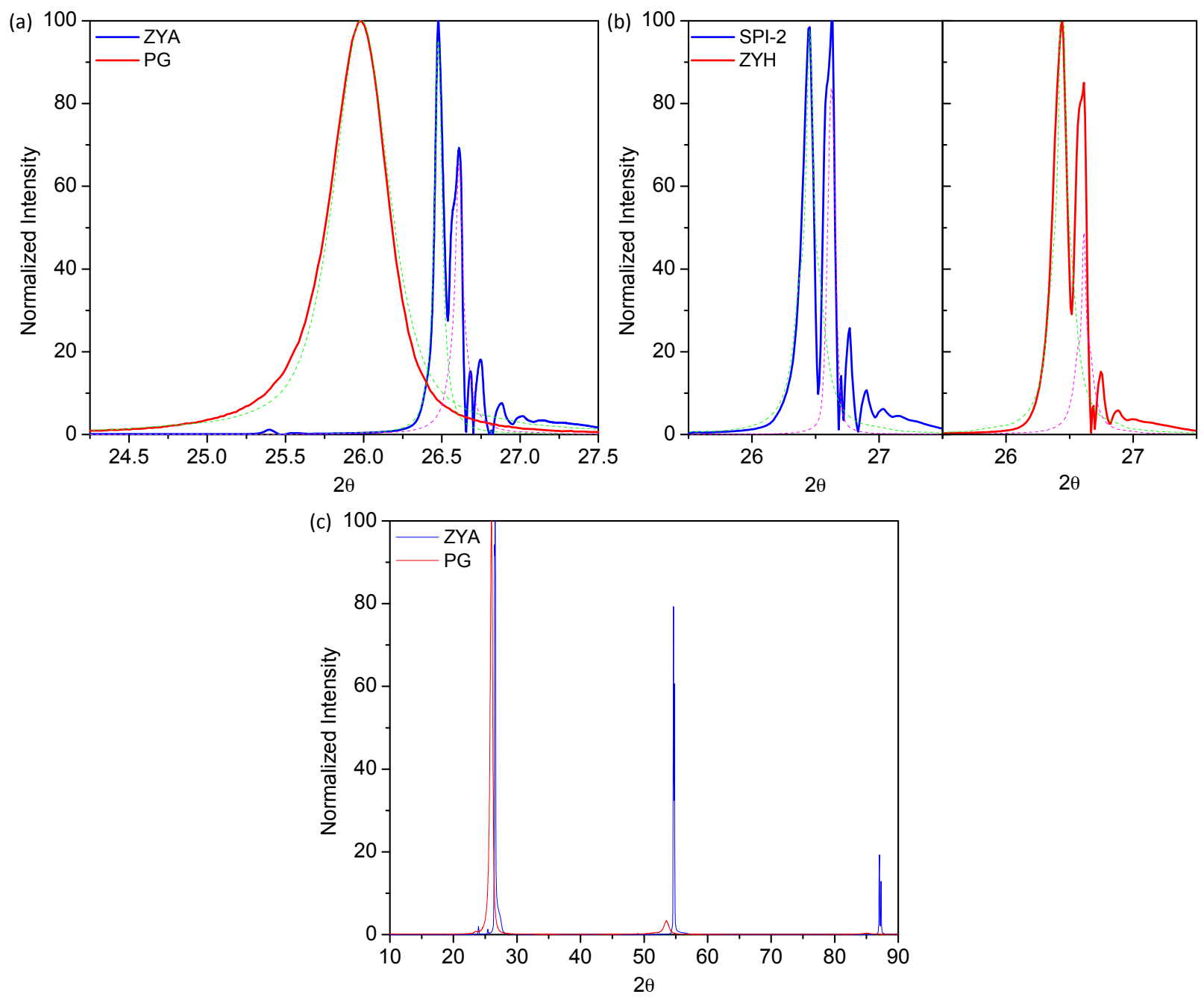

Figure 31. XRD spectra of freshly exfoliated graphite. (002) rocking curve for (a) ZYA and PG and (b) SPI-2 and ZYH. (c) Entire spectrum for ZYA and PG; spectrum for SPI-2 and ZYH is similar to the spectrum of ZYA. High resolution spectra were collected from $23^{\circ}-30^{\circ} 2 \theta$ with 0.002 increments and $0.3 \mathrm{sec} /$ increment. K $\alpha 2$ stripping was performed before peak fitting and dotted lines indicate peak fitting (parameters shown in Table 22). 


\section{APPENDIX C}

\section{ELUCIDATING SURFACE CHEMISTRY BY XPS}

\section{C.1 SURFACE CHEMISTRY}

XPS was performed on the graphite samples to determine surface chemistry and verify $\mathrm{sp}^{2}$ carbon hybridization. The focus of these experiments was to elucidate any chemical differences between the highest and lowest quality graphite samples. ZYA was cleaved in air using 1-inch Scotch tape, mounted to the sample holder, and placed into the sample preparation chamber within 30 seconds of exfoliation. PG was prepared similarly except the sample was cleaved with a razor blade to create a fresh surface. Testing was performed using a Thermo Scientific ESCALAB 250Xi X-ray Photoelectron Spectrometer (XPS) Microprobe with monochromated Al K $\alpha$ X-ray source $(1486.6 \mathrm{eV}$ ) and $200 \mu \mathrm{m}$ spot size (Appendix D discusses data collected at $900 \mu \mathrm{m}$ spot size). A low-energy electron source supplied charge compensation.

The following procedure was followed for all XPS experiments: (1) The survey spectrum was collected for 10 scans (pass $=150 \mathrm{eV}$ ) followed by high resolution (2) valence spectrum for 20 scans (pass $=50 \mathrm{eV}),(3) \mathrm{C} 1 \mathrm{~s}$ spectrum for 10 scans (pass=50 eV), (4) $\mathrm{O} 1 \mathrm{~s}$ spectrum for 20 scans (pass $=50 \mathrm{eV}$ ), and (5) $\mathrm{C}$ KLL Auger spectrum for 10 scans (pass=50 eV). Thermo 
Avantage software was used for spectra acquisition and peak fitting was performed using OriginPro. Data was collected at $0^{\circ}$ where the photoelectron emission and surface normal are equal $\left(90^{\circ}\right.$ takeoff angle). Angle Resolved-XPS (AR-XPS) was used to identify surface and bulk components. AR-XPS data were collected at $0^{\circ}$ (more bulk sensitive) and $60^{\circ}$ (more surface sensitive). ${ }^{336}$ Each spectrum is the average of separate experiments as indicated. The standard deviation of spectra from different experiments was very small and the average spectra is representative of the individual experiment. The only exception is $\mathrm{O} 1 \mathrm{~s}$ due to the very small amount of oxygen present on the sample. All data are normalized to the highest peak.

Figure 32 shows XPS data of ZYA and PG. Survey spectra show no discernible differences. There is a strong carbon peak at $284.3 \mathrm{eV}$ and a slight oxygen peak at $532.0 \mathrm{eV}$. Additionally there are valence peaks near $0 \mathrm{eV}$ and C KLL Auger peaks near $1200 \mathrm{eV}$. Peak fitting of the survey spectrum shows that carbon accounts for $c a .99 .6$ atomic percent (at $\%$ ) and oxygen accounts for ca. 0.4 at $\%$ for ZYA. Ashraf et al. reported a similar oxygen value, 0.60 at $\%$, for SPI-1 (comparable quality to ZYA) HOPG cleaved in air. ${ }^{230}$ In comparison, carbon accounts for $c a .99 .1$ at $\%$ and oxygen accounts for $c a .0 .9$ at $\%$ for PG. High resolution valence (Figure 32b) and C1s (Figure 32c) scans appear to show no significant difference between the two samples. The valence spectra have a peak stretching from $15-20 \mathrm{eV}$ with a slight shoulder at $12.5 \mathrm{eV}$. The featureless peak is indicative of a defect free graphitic surface. ${ }^{337}$ The flat, broad peak at $15-20 \mathrm{eV}$ is distinctly characteristic of graphite; whereas, $\mathrm{sp}^{3}$-hybridized carbon produces a sharp peak as first characterized by Shirley et $a .^{338,} 339$ and confirmed through DFT calculations. ${ }^{340}$ Deconvolution of the $\mathrm{C} 1 \mathrm{~s}$ spectra is rigorous and will be explored in detail (vide infra). 
Interestingly, the high resolution O1s spectra (Figure 33) are distinctly different. The peaks were fit with a single symmetric Gaussian function to quantify the peak area and amount of oxygen in each sample. The background was taken as a constant value from the average of endpoints and subtracted from the spectrum. The calculated peak area of ZYA and PG is 0.110 \pm 0.004 and $0.191 \pm 0.004$, respectively, indicating that there is $c a .75 \%$ more oxygen on the PG sample. This difference appears significant, however, the large error of the spectra when averaged from the six experiments (Figure 33a) indicates that there is variability between different surface sites. The variability is unsurprising since oxygen is not intrinsic to graphite and adsorbs at both defect sites and on the basal plane. ${ }^{230,241,317,341,342}$ Investigations on graphene doping show that molecular oxygen, organic molecules, and water vapor adsorb at edge sites, surface defects, and the basal plane in a manner which can also occur on graphite. ${ }^{343-}$ 345 The amount of oxygen detected in the survey scan (Figure 32a) is ca. 0.4-0.9 at $\%$ indicating that the overall contribution of oxygen to the spectrum is small. Nevertheless, both survey and O1s scans show that PG has about twice the amount of surface oxygen as ZYA.

C KLL Auger peaks acquired through XPS are shown in Figure 34. Following the spectra from low to high binding energy, both curves begin to increase at $1202 \mathrm{eV}$ and peak at $1218.6 \mathrm{eV}$. At this point, ZYA takes a slight dip then increases to peak at $1227.4 \mathrm{eV}$ while PG continues to decrease. Both spectra then follow the same path towards increasing binding energy. The only difference between the spectra is the peak location at $1227.4 \mathrm{eV}$ for ZYA and 1218.6 eV for PG. Presence of these peaks, opposed to a featureless, rounded curve, indicates that the samples have very low defect density. ${ }^{346}$ These features become less pronounced as 
defects and disorder are introduced to the sample as shown by Speranza and Laidani in which they irradiated HOPG surface with $\mathrm{Ar}^{+}$ions for increasing amount of time and observed that the features disappeared with increasing exposure to damaging $\mathrm{Ar}^{+}$ions. ${ }^{346}$
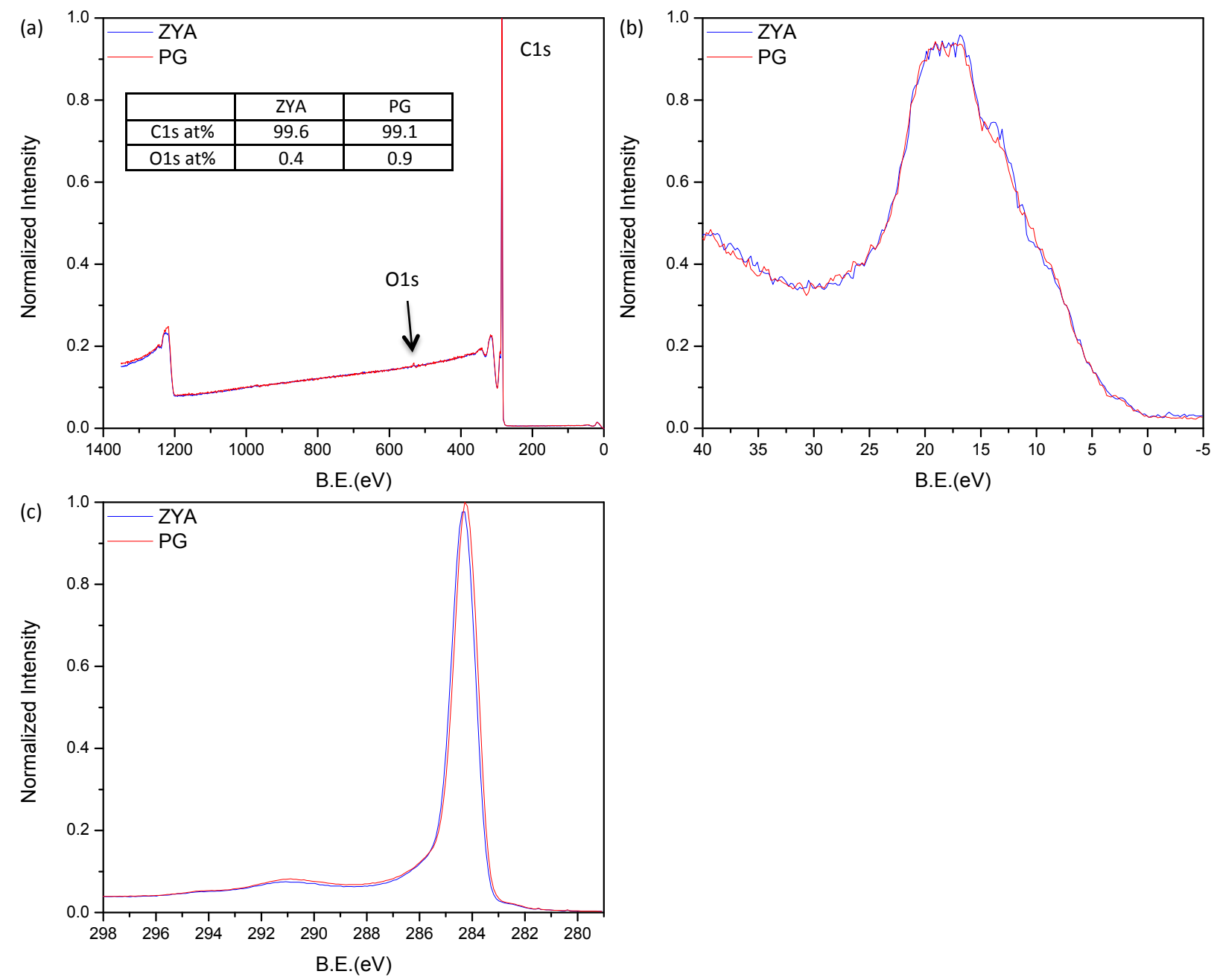

Figure 32. XPS spectra of graphite samples. (a) Survey, (b) valence, and (c) high resolution C1s spectra of ZYA and PG. Each spectrum is the average of six separate experiments. 

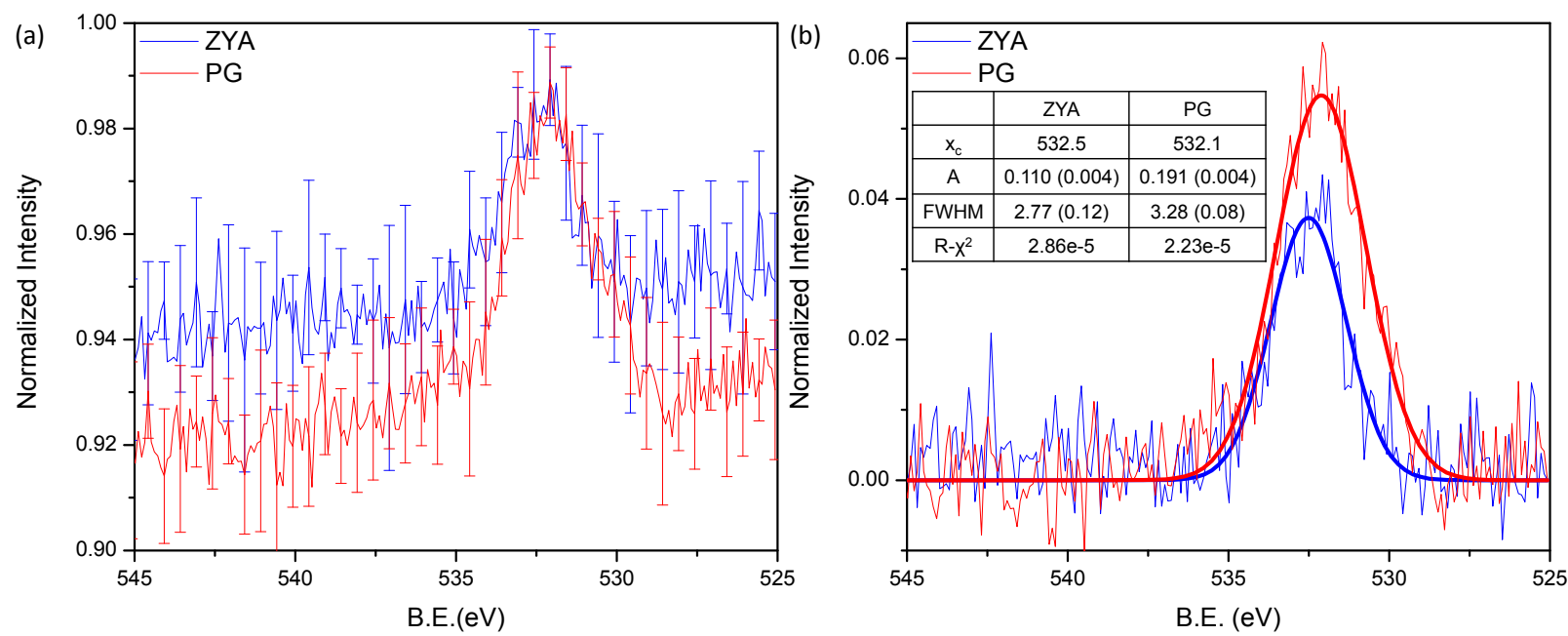

Figure 33. High resolution O1s spectra of ZYA and PG. Each spectrum is the average of six separate experiments and error bars indicate standard deviation. Inset of Figure 33b shows Gaussian function fitting results.

C KLL Auger spectra can be used to identify carbon hybridization by calculating the Dparameter. $^{347,} 348$ The D-parameter is simply the binding energy difference between the maximum and minimum peaks of the differentiated $\mathrm{x}$-ray C KLL spectra. Diamond is $100 \% \mathrm{sp}^{3}$ hybridized and has a D-parameter of 13 . Graphite is $100 \% \mathrm{sp}^{2}$-hybridized and has a D-parameter of 21. Interestingly, the relationship of $\mathrm{D}$-parameter on $\mathrm{sp}^{2} / \mathrm{sp}^{3}$ hybridization is linear and can be interpolated to determine the relative amount of hybridization of a carbon sample. ${ }^{337,349-352}$ Taking the first derivative of the spectra in Figure 34a yields Figure 34b for ZYA and Figure 34c for PG. The D-parameter can be directly estimated from the graphs and was determined to be 22.5 for both ZYA and PG. The vertical lines indicate the position of maximum $(1212 \mathrm{eV})$ and minimum $(1234.5 \mathrm{eV})$ peaks which were the same for both samples. Deviance of the experimental D-parameter from its theoretical value of 21 for $\mathrm{sp}^{2}$-hybridized carbon is attributed to sample variability. In fact, reported values for graphite and HOPG range from $21.1^{353}$ to 22.8 $\mathrm{eV}^{337,351,354,355}$ Our 22.5 value is nearly identical to the 22.6 value reported by Popov et al. ${ }^{355}$ 
This result indicates unambiguously that both samples are $\mathrm{sp}^{2}$-hybridized carbon. Considering this XPS data along with Raman and XRD results, we can conclude that both samples are high quality $\mathrm{sp}^{2}$-hybridized graphite with relatively little surface defects.
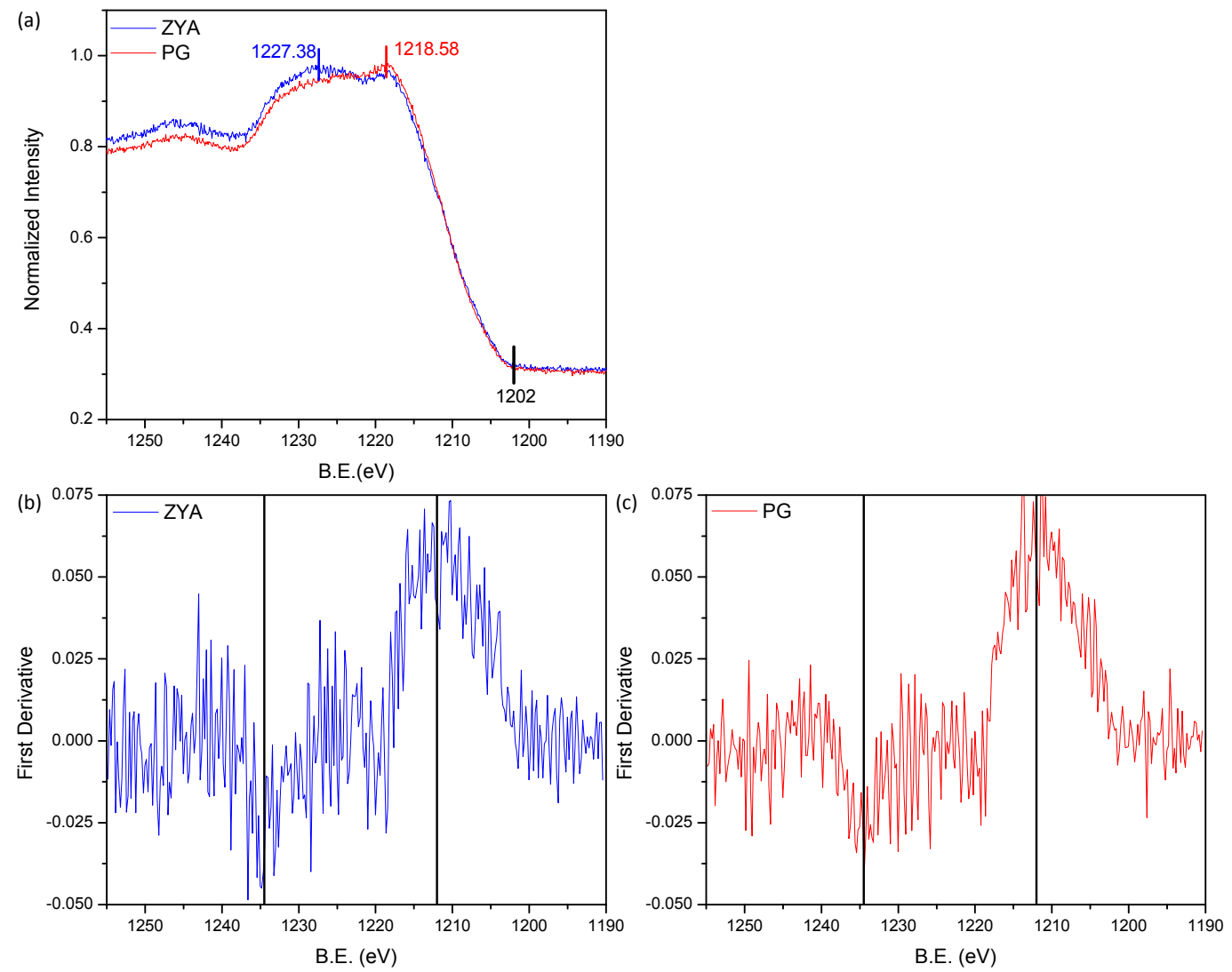

Figure 34. C KLL Auger spectra of graphite samples. (a) High resolution spectra of ZYA and PG. Each spectrum is the average of six separate experiments. First derivative of the (b) ZYA and (c) PG spectra in Figure 34a. The vertical lines indicate the maximum and minimum peak position and the binding energy difference between these two points is the D-parameter. 


\section{C.2 C1s PEAK FITTING METHODOLOGY}

There is no standard method for fitting the C1s spectra of graphitic materials and researchers have developed numerous methods to describe the asymmetric peak. Methods have ranged from (a) deconvoluting the main peak into nine separate symmetric peaks, ${ }^{356}$ (b) describing the peak at $284.4 \mathrm{eV}$ with a single Doniach-Sunjic function, ${ }^{357-360}$ and (c) fitting the surface and bulk components separately. ${ }^{335,358,361}$ Each method has inherent complexity and provides different information. For example, the Doniach-Sunjic function describes the asymmetry $(\alpha)$ of the main peak by using convoluted Gaussian/Lorentzian functions. Satellite and other peaks are concomitantly fit as a Gaussian/Lorentzian mix. Contrarily, deconvoluting into nine separate peaks allows for a more precise fit although several of the peaks become very small and there is risk of overfitting the spectrum.

Several researchers have investigated the physical meaning of the asymmetry parameter ( $\alpha$ ) by comparing graphite samples with varying degree of ion induced defects and fitting the

resulting spectra. ${ }^{346,360,362-364}$ Peak asymmetry (and asymmetry parameter $(\alpha)$ in the DoniachSunjic equation) correlates to the degree of defects within the sample where perfect $\mathrm{sp}^{2}$ carbon would have a single symmetric peak and $\alpha$ increases with amount of defects. ${ }^{360}$ That is, asymmetry correlates to the amount of surface defects. This approach is beneficial for understanding how the degree of surface defects affect the spectra. $\alpha$ provides quantitative defect information but does not provide information on the type of defects or constituent peaks of the main asymmetric peak.

ZYA and PG spectra were fit using the Doniach-Sunjic function for C1-C3 and 80\% Gaussian PsdVoigt1 for C4-C5 (vide infra). Section A.4 on page 178 has further details along 
with fitting results. Foremost, the Doniach-Sunjic asymmetry parameter $(\alpha)$ was similar for both ZYA and PG: 0.07 and 0.08, respectively, which is similar to previously reported values for HOPG and natural graphite. ${ }^{358,365-367}$ Considering that previous work intentionally irradiated the HOPG surface with ions to induce asymmetry of the $\mathrm{C} 1$ peak $(284.3 \mathrm{eV}){ }^{360}$ the results of this study conclude that intrinsic defects of high quality samples do not manifest with significant asymmetry; therefore, quantifying intrinsic defects by $\alpha$ is not possible.

Accurately fitting the C1s spectra of ZYA and PG is critical for elucidating any possible chemical differences between the two samples; therefore, five peak fitting functions were tried which deconvolute the main peak into five symmetric peaks. The background was first removed by the Shirley method followed by fitting each of the five peaks (C1-C5; Table 23) with the Gaussian, Gauss, Lorentzian, PsdVoigt1, or PsdVoigt2 function in OriginPro. Appendix D.2 (page 170) provides details of each function along with fitting results. Literature suggests that the peaks are primarily Gaussian and partially Lorentzian and the peaks were systematically fit to determine which model provided the best results. ${ }^{357,358,363,364}$

After methodically fitting the spectra by the different functions, this study ultimately relies on the PsdVoigt1 function:

$$
y=y_{0}+A\left[\mu \frac{2}{\pi} \frac{F W H M}{4\left(x-x_{C}\right)^{2}+F W H M^{2}}\right]+(1-\mu) \frac{e^{\frac{-4 \ln (2)\left(x-x_{C}\right)^{2}}{F W H M^{2}}}}{F W H M \sqrt{\frac{\pi}{4 \ln (2)}}} \quad \text { Equation } 57
$$

PsdVoigt1 is a linear combination of the Lorentzian and Gaussian functions where both terms have the same full width at half maximum (FWHM) value. ${ }^{368}$ The peak center $\left(\mathrm{x}_{\mathrm{c}}\right)$ was fixed 
according to Table 23 and $\mu=0$ for $\mathrm{C} 1-\mathrm{C} 2$ and $\mu=0.2$ for $\mathrm{C} 3-\mathrm{C} 5$, where the function is $100 \%$ Gaussian when $\mu=0$ and $100 \%$ Lorentzian when $\mu=1$. The peak amplitude (A; area) and FWHM were fit while the y-offset $\left(\mathrm{y}_{0}\right)$ was fixed at 0 .

\section{C.2.1 Fitting the C1s spectra}

The C1s spectra shown in Figure 35 has a prominent peak at $284.3 \mathrm{eV}$ characteristic of $\mathrm{sp}^{2}-$ hybridized carbon. $338,339,356,360,369,370$ This peak is asymmetric and becomes more asymmetric towards higher binding energy. This asymmetry originates from surface defects on the $\mathrm{sp}^{2}$ carbon lattice $357,360,370$ and necessitates deconvolution by several individual, symmetric peaks. The C1s spectra also has a satellite peak at $291 \mathrm{eV}$. Yang and Sacher presented a method to fit the C1s spectra using five constituent peaks whose attributes are shown in Table $23 .^{360,363,364}$ This method was chosen because it provided an appropriate level of detail into the chemical nature of the $\mathrm{C} 1 \mathrm{~s}$ peak allowing for differentiation between $\mathrm{sp}^{2}$ carbon and surface defects without overfitting the spectrum. The final results of fitting the ZYA and PG spectra are shown in Figure 35c and Figure 35d, respectively. See Section A.3 on page 177 for comments on fitting error.

Delocalized $\mathrm{sp}^{2}$ carbon $(\mathrm{C} 1)$ accounts for $60-65 \%$ of the $\mathrm{C} 1 \mathrm{~s}$ spectrum with the remainder being (C2) localized $\mathrm{sp}^{2}$ carbon indicating defects and damage, (C3) free radical $\mathrm{sp}^{3}$ and $\mathrm{C}-\mathrm{O}-\mathrm{C}$, (C4) $\pi \Rightarrow \pi^{*}$ shakeup of $\mathrm{C} 2$, and (C5) $\pi \Rightarrow \pi^{*}$ shakeup of $\mathrm{C} 1 .{ }^{360,363}$ The data in Table 23 shows that there are slight differences between ZYA and PG. The delocalized $\mathrm{sp}^{2}$ carbon indicative of graphite has an at $\%$ of 65.6 and 59.1 for ZYA and PG, respectively. Asymmetry of the large peak centered at $284.3 \mathrm{eV}$ is caused by $\mathrm{C} 2$ and C3: ZYA has a smaller amount of defects 
resulting in 16.8 at\%; whereas, PG has more defects resulting in 25.7 at\%. C4-C5 are similar for both samples.

Since C4-C5 are shakeup satellites and do not directly contribute to the main peak asymmetry, they were subtracted from the $\mathrm{C} 1 \mathrm{~s}$ spectra then $\mathrm{C} 1-\mathrm{C} 3$ were refit to determine their relative contribution. Figure 36 shows the $\mathrm{C} 1$ s spectra with only $\mathrm{C} 1-\mathrm{C} 3$ fit using the parameters in Table 23. The two peaks appear similar (Figure 36a) yet they result in different peak fits. Fitting PG to the exact same parameters as ZYA yielded a poor fit with large error. PG required shift of the $\mathrm{C} 2$ peak center $\left(\mathrm{x}_{\mathrm{c}}\right)$ by $0.1 \mathrm{eV}$ to provide a good fit. The error of the fit shown in Figure $36 \mathrm{~b}$ is $1-3 \%$ with greatest error around the $\mathrm{C} 1$ peak. $\mathrm{C} 2$ contributes the most to asymmetry with 13.6 at $\%$ and 23.7 at $\%$ in ZYA and PG, respectively. PG has about $75 \%$ more C2 contribution than ZYA. Moreover, C3 plays a more significant role in ZYA. This can be because $\mathrm{O}_{2}$ adsorbs at both defect sites and on the basal plane. ${ }^{230,241,317,341,342}$ ZYA has more basal surface while PG has more defect sites. Part of this difference may also be due to peak fitting since there is some inherent variability when fitting small peaks. Additionally, adsorbed contaminants can contribute to this behaviour since the sample is exfoliated in ambient air and transferred to the XPS preparation chamber which is then pumped down to UHV ( $\sim 20 \mathrm{~min})$. Contaminants will inevitably adsorb to the graphite surface upon exfoliation in air and subsequent pumpdown (see Section C.4 on page 165). Overall, the data indicates that ZYA has more pristine $\mathrm{sp}^{2}$ carbon and less defects.

Mangolini et al. used near-edge x-ray absorption fine structure (NEXAFS) spectroscopy and XPS to investigate the thickness and chemical nature of the adsorbed contamination layer on diamond and amorphous carbon. They reported that the contaminant layer has a thickness of 0.6 $\pm 0.2 \mathrm{~nm}$ and consists of $19 \pm 3 \% \mathrm{sp}^{2}$ carbon. Moreover, they report the ratio of adsorbed 
oxygen-to-carbon to be $0.11 \pm 0.02 .^{236}$ Localized $\mathrm{sp}^{2}$ carbon is indicative of contamination and was determined to be 13.6 at $\%$ and 23.7 at $\%$ on ZYA and PG, respectively (Figure $36 \mathrm{c}$ ). These values agree fairly well to the value reported by Mangolini et al. claiming that the contaminant layer consists of 19 at $\% \mathrm{sp}^{2}$ carbon. Furthermore, ZYA had 7.5 at $\%$ of C-O-C (and sp ${ }^{3}$ ) bonding which is fairly similar to the $11 \%$ oxygen-to-carbon ratio. These results show agreement between two complimentary methods and may further be refined for investigating what exactly adsorbs onto fresh graphitic surfaces.

Table 23. Peak attribution and fitting results for the C1s spectra of ZYA and PG. Spectra are the average of eleven separate experiments (Figure 35) and fitting results are presented as the average at $\%$ (standard deviation) using the PsdVoigt l function. Reduced- $\chi^{2}$ of ZYA and PG fit is $3.23 \times 10^{-5}$ and $4.27 \times 10^{-5}$, respectively.

\begin{tabular}{|c|c|c|c|c|c|c|c|}
\hline \multirow[b]{2}{*}{ Peak } & \multirow[b]{2}{*}{$\begin{array}{l}\text { Peak Center } \\
\left(\mathrm{x}_{\mathrm{c}}\right)^{360,363} \\
(\mathrm{ZYA} / \mathrm{PG})\end{array}$} & \multirow{2}{*}{$\begin{array}{c}\mu \\
0=100 \% \\
\text { Gaussian } \\
1=100 \% \\
\text { Lorentzian }\end{array}$} & \multirow[b]{2}{*}{ Attribute ${ }^{360,363}$} & \multicolumn{2}{|c|}{ ZYA } & \multicolumn{2}{|c|}{ PG } \\
\hline & & & & atomic\% $\%$ & FWHM & atomic\% & FWHM \\
\hline $\mathrm{C} 1$ & $284.3 \mathrm{eV}$ & 0 & $\begin{array}{l}\text { delocalized } \mathrm{sp}^{2} \\
\text { carbon }\end{array}$ & $\begin{array}{l}65.6 \\
(3.0)\end{array}$ & $\begin{array}{c}0.997 \\
(0.004)\end{array}$ & $\begin{array}{l}59.1 \\
(1.9)\end{array}$ & $\begin{array}{c}1.000 \\
(0.005)\end{array}$ \\
\hline $\mathrm{C} 2$ & $285.1 / 285.0 \mathrm{eV}$ & 0 & $\begin{array}{l}\text { localized } \mathrm{sp}^{2} \text { carbon } \\
\text { (defects, damage, } \\
\text { and contamination) }\end{array}$ & $\begin{array}{l}11.3 \\
(4.1)\end{array}$ & $\begin{array}{c}1.385 \\
(0.075)\end{array}$ & $\begin{array}{l}18.4 \\
(2.0)\end{array}$ & $\begin{array}{l}2.813 \\
(0.331)\end{array}$ \\
\hline $\mathrm{C} 3$ & $286.3 \mathrm{eV}$ & 0.2 & $\begin{array}{l}\text { free radical } \mathrm{sp}^{3} \& \\
\qquad \mathrm{C}-\mathrm{O}-\mathrm{C}\end{array}$ & $\begin{array}{c}5.5 \\
(2.2)\end{array}$ & $\begin{array}{c}1.606 \\
(0.204)\end{array}$ & $\begin{array}{c}7.3 \\
(2.1)\end{array}$ & $\begin{array}{c}6.010 \\
(1.010)\end{array}$ \\
\hline $\mathrm{C} 4$ & $287.7 / 287.5 \mathrm{eV}$ & 0.2 & $\pi \Rightarrow \pi^{*}$ shakeup of $\mathrm{C} 2$ & $\begin{array}{c}3.0 \\
(1.6)\end{array}$ & $\begin{array}{c}2.666 \\
(0.651)\end{array}$ & $\begin{array}{c}1.6 \\
(1.8)\end{array}$ & $\begin{array}{l}6.001 \\
(2.934)\end{array}$ \\
\hline $\mathrm{C} 5$ & $291.0 / 290.9 \mathrm{eV}$ & 0.2 & $\pi \Rightarrow \pi^{*}$ shakeup of $\mathrm{C} 1$ & $\begin{array}{l}14.7 \\
(1.7)\end{array}$ & $\begin{array}{c}5.219 \\
(0.253)\end{array}$ & $\begin{array}{l}13.7 \\
(0.7)\end{array}$ & $\begin{array}{c}5.474 \\
(0.543)\end{array}$ \\
\hline
\end{tabular}



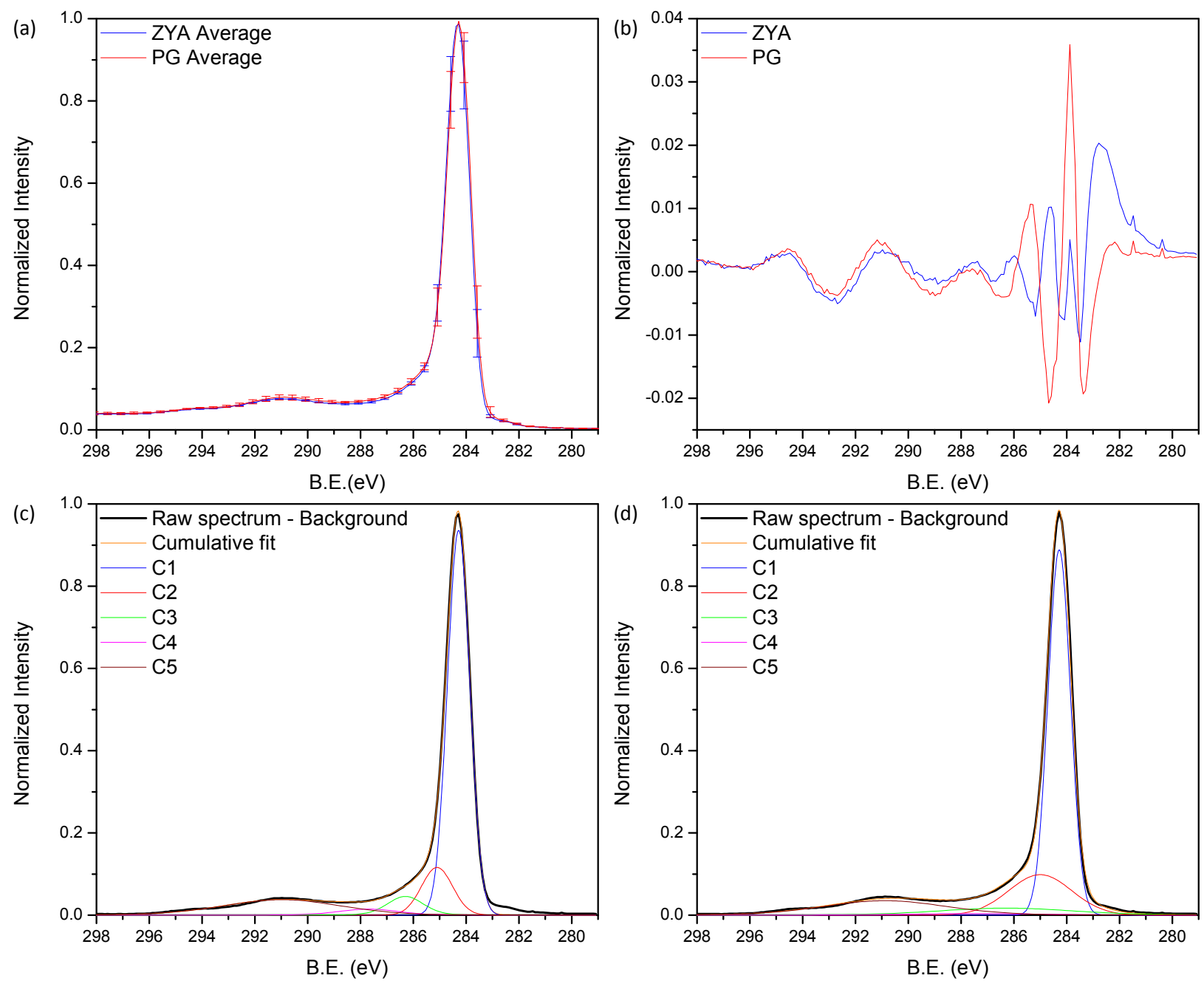

Figure 35. High resolution C1s spectra of ZYA and PG. (a) Each spectrum is the average of eleven separate experiments and error bars indicate standard deviation. (b) Residual/Error of

PsdVoigt1 model. (c) ZYA and (d) PG spectrum fit with five symmetric peaks. Shirley background was subtracted from raw data and peaks were fit with PsdVoigtl function with $\mu=0$ for $\mathrm{C} 1-\mathrm{C} 2$ and $\mu=0.2$ for $\mathrm{C} 3-\mathrm{C} 5$. 

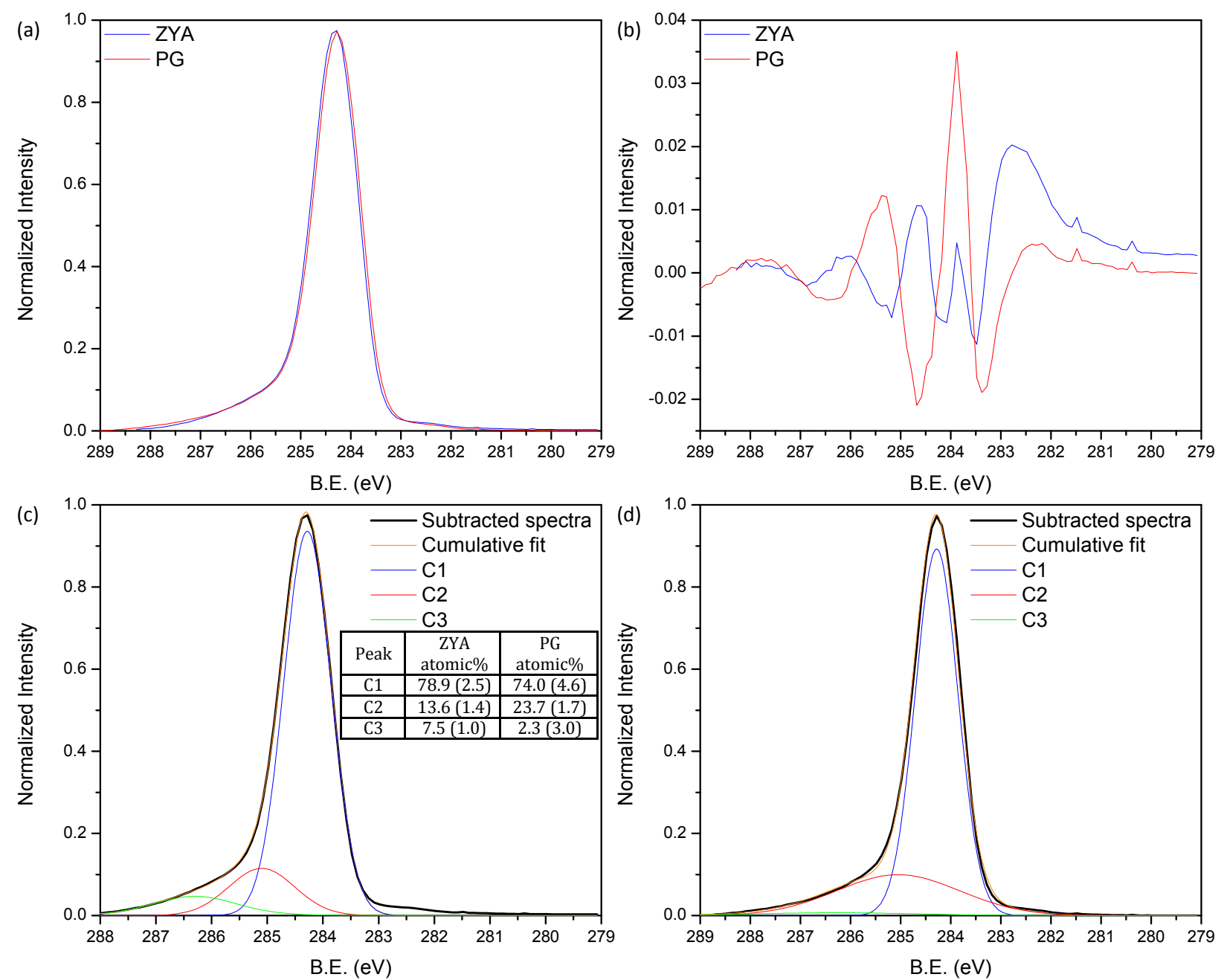

Figure 36. High resolution C1s spectra where only peaks $\mathrm{C} 1-\mathrm{C} 3$ are fit. (a) The data is taken from Figure 35 and the $\mathrm{C} 4-\mathrm{C} 5$ peaks are subtracted from the spectrum then $\mathrm{C} 1-\mathrm{C} 3$ are fit. (b) Residual/Error of PsdVoigt1 model. (c) ZYA spectrum with inset showing fitting results and (d) PG spectrum. Reduced- $\chi^{2}$ for ZYA and PG is $6.21 \times 10^{-5}$ and $7.28 \times 10^{-5}$, respectively.

\section{C.3 ANGLE RESOLVED XPS (AR-XPS)}

AR-XPS data was obtained at $0^{\circ}$ and $60^{\circ}$ (grazing angle) to determine differences in the bulk and surface components of ZYA and PG. Figure 37 shows the spectra along with fitting results for O1s. ZYA showed an $87 \%$ increase of oxygen at grazing angle while PG showed a $50 \%$ increase. This indicates that the oxygen contaminants are located primarily at the uppermost 
graphite surface which is in contact with air. It is important to note that the oxygen increase for ZYA is substantially greater than the oxygen increase for PG. If oxygen were solely on the surface for both ZYA and PG, the increase would be similar, i.e., closer to the value for ZYA. The diminished oxygen increase for PG indicates that oxygen resides both at the sample surface and within the sample, possibly intercalated during storage or residual from sample synthesis. This hypothesis is further supported by Raman spectroscopy which indicates that PG is turbostratic with no (or weak) interlayer coupling and XRD data (Appendix B) indicating that the interlayer spacing of PG is $3.43 \AA$ which is greater than the interlayer spacing of graphite (3.35 A). ${ }^{9,} 11$ This may allow for oxygen to intercalate and reside between individual graphene layers; whereas, strong interlayer coupling in ZYA minimizes opportunity for oxygen to intercalate. Moreover, PG has $44 \%$ and $15 \%$ more oxygen than ZYA at $0^{\circ}$ and $60^{\circ}$, respectively. After

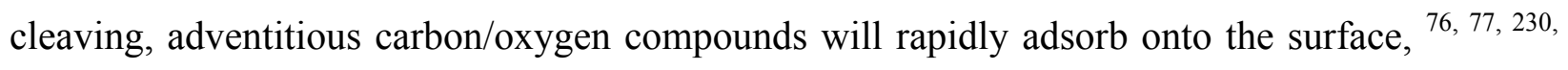
${ }^{241}$ and these compounds, along with atmospheric oxygen, adhere at graphite surface sites. ${ }^{230,241,}$ 317, 341-345 This is qualitatively consistent with Figure 33 showing that PG has greater oxygen content, thus more defects.

The C1s spectra were fit using the PsdVoigt1 function with peaks attributed according to Table 23 and the at $\%$ for ZYA and PG are shown in Table 24 . When error is considered in the data, there is no significant change in at $\%$ for any of the peaks as the test becomes more surface sensitive. Based on the origin of photoelectrons, the original hypothesis was that $\mathrm{C} 1$ would decrease and $\mathrm{C} 2-\mathrm{C} 3$ would increase with grazing angle; however, this clearly was not the case. $\mathrm{C} 1$ occurs within each layer of graphene, thus it is a bulk property and its contribution is expected to decrease with grazing angle. $\mathrm{C} 2$ is localized $\mathrm{sp}^{2}$ carbon due to defects. These defects are present within each layer of the sample but are more prominent on the surface due to 
exfoliation (see Section 4.2.2 on page 71), thus it is more surface sensitive and should increase. Similarly, C3 is $\mathrm{sp}^{3}$ carbon radical or C-O bonding. Since the bulk graphite is considered to be pristine and oxygen adsorbs at the surface, the $\mathrm{C} 3$ peak results from surface electrons and should increase with grazing angle. C4-C5 are $\pi \Rightarrow \pi^{*}$ shakeup satellites and the electrons originate throughout the entire sample; therefore, their change should be related to $\mathrm{C} 1$ and $\mathrm{C} 2$.

AR-XPS can not provide any quantitative information on defect density because the surface sensitivity of the test is not high enough (see Section C.4 on page 165). Also, the surface is not homogeneous because defects are randomly distributed and contaminant adsorption occurs at both the basal plane and defect sites. The adsorption can not be well controlled because it is a function of the specific surface being tested and the amount of hydrocarbons in air. This leads to a relatively large error as shown in Table 24. The variability is emblematic of AR-XPS data in general.

A feature of the AR-XPS data is the $\mathrm{C} 1$ peak shift towards higher binding energy as angle increases. As the test becomes more surface sensitive, surface defects become more prominent and cause a shift in the electron localization which manifests as a shift towards higher binding energy. ${ }^{358,360}$ Peak asymmetry also increases with grazing angle since surface defects have a greater contribution to the spectrum. ${ }^{358,361}$ In conclusion, ZYA and PG are $\mathrm{sp}^{2}$-hybridized carbon $(\mathrm{C} 1$ peak centered at $284.3 \mathrm{eV})$ with few surface defects and a small amount of adsorbed oxygen on the surface. Similar to previous results at $0^{\circ}$ (Figure 36 and Table 23), ZYA has a greater amount of pristine $\mathrm{sp}^{2}$ carbon (C1), less defects (C2-C3), and less adsorbed oxygen (O1s). 
Table 24. Fitting results of AR-XPS C1s spectra. Spectra is average of three separate experiments (Figure 37) and fitting results are presented as the average at\% (standard deviation) using the PsdVoigtl function.

\begin{tabular}{|c|c|c|c|c|}
\hline \multirow{2}{*}{} & \multicolumn{2}{|c|}{ ZYA } & \multicolumn{2}{c|}{ PG } \\
\cline { 2 - 5 } & $0^{\circ}$ & $60^{\circ}$ & $0^{\circ}$ & $60^{\circ}$ \\
\hline \multirow{2}{*}{ C1 } & $\mathbf{6 6 . 3}$ & $\mathbf{6 3 . 4}$ & $\mathbf{6 2 . 4}$ & $\mathbf{6 0 . 0}$ \\
& $\mathbf{( 5 . 1 )}$ & $\mathbf{( 4 . 3 )}$ & $\mathbf{( 0 . 6 )}$ & $\mathbf{( 2 . 5 )}$ \\
\hline \multirow{2}{*}{ C2 } & 12.7 & 11.9 & 16.6 & 14.1 \\
& $(8.8)$ & $(7.8)$ & $(4.0)$ & $(0.8)$ \\
\hline \multirow{2}{*}{ C3 } & 3.9 & 4.4 & 2.4 & 4.2 \\
& $(3.4)$ & $(3.1)$ & $(1.3)$ & $(0.5)$ \\
\hline \multirow{2}{*}{ C4 } & 2.0 & 3.1 & 4.1 & 9.9 \\
& $(1.8)$ & $(1.3)$ & $(1.1)$ & $(8.5)$ \\
\hline \multirow{2}{*}{ C5 } & 15.1 & 17.1 & 14.5 & 11.8 \\
& $(1.5)$ & $(7.9)$ & $(3.3)$ & $(6.3)$ \\
\hline \multirow{2}{*}{ R- $\chi^{2}$} & $2.27 \mathrm{e}-4$ & $8.37 \mathrm{e}-5$ & $5.32 \mathrm{e}-5$ & $4.53 \mathrm{e}-5$ \\
\hline
\end{tabular}



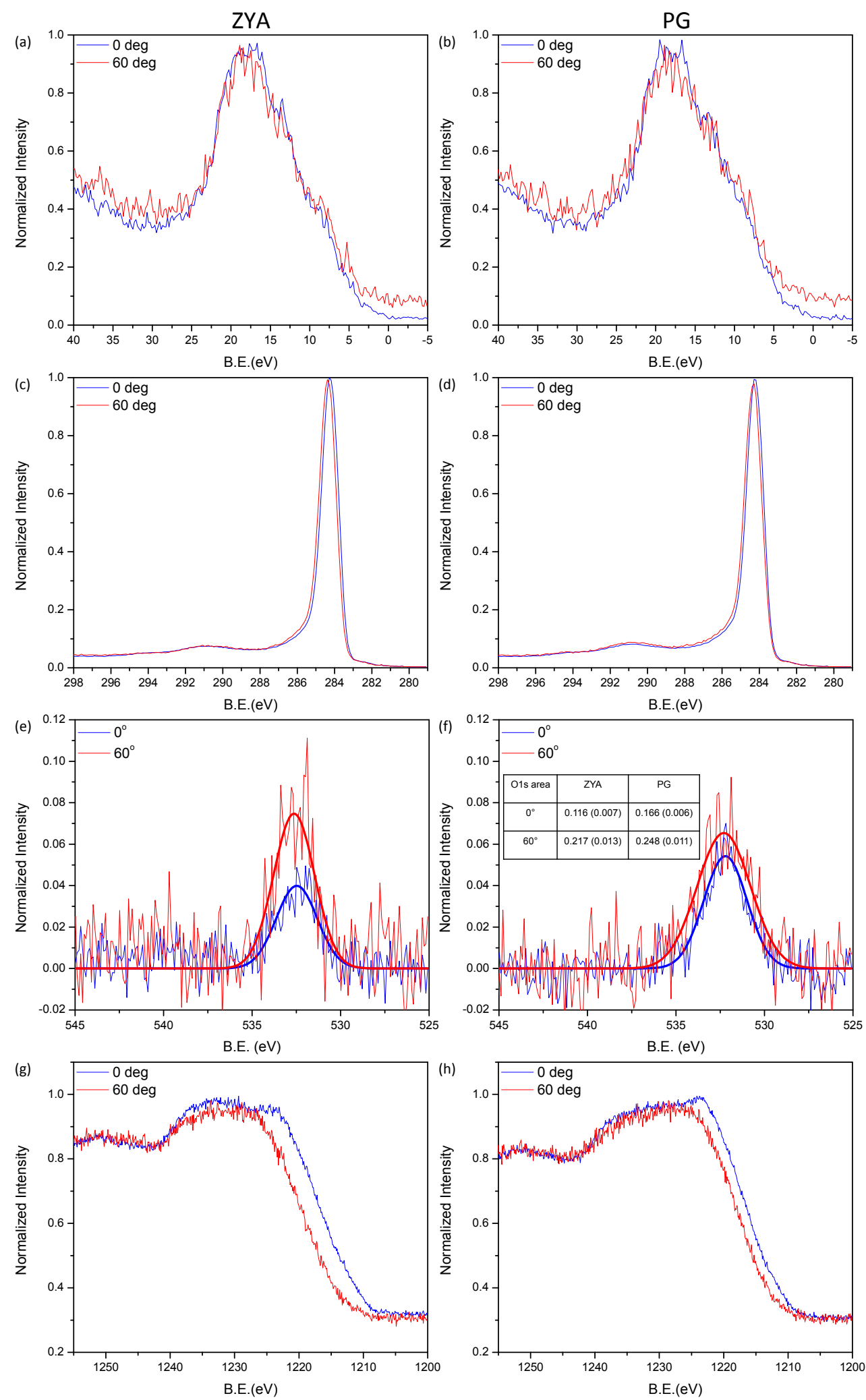

Figure 37. AR-XPS spectra of ZYA and PG. Each spectrum is the average of two separate experiments. O1s spectra were fit using a Gaussian function and inset shows peak area. 


\section{C.4 ATTENUATION AND EFFECTS OF CONTAMINATION}

XPS is a quasi-surface sensitive technique with a penetration depth $c a .8 .7 \mathrm{~nm}$ in HOPG (at $90^{\circ}$ takeoff angle). ${ }^{335}$ The number of sampled graphene layers $\left(\mathrm{N}_{\mathrm{GL}}\right)$ can be calculated using the interlayer spacing (d) of HOPG:

$$
N_{G L}=\frac{8.7 \mathrm{~nm}}{0.336 \mathrm{~nm}}=25.9 \quad \text { Equation } 58
$$

where $\mathrm{d}=0.336 \mathrm{~nm}$ for ZYA as determined by $\mathrm{x}$-ray diffraction (Table 22 on page 146). The relative intensity of photoelectrons detected from the surface $\left(I_{S}\right)$ is equal to $1 / \mathrm{N}_{\mathrm{GL}}$, thus the surface contribution to the overall XPS signal is $3.9 \%$ and the bulk contribution $\left(I_{B}\right)$ is $96.1 \%$. ${ }^{335}$

An alternative approach is to calculate $\mathrm{I}_{\mathrm{S}}$ using the exponential decay equation:

$$
\frac{I_{S}}{I_{B}}=e^{|d / \lambda \sin \theta|}-1 \quad \text { Equation } 59
$$

where $\lambda$ is the inelastic mean free path (IMFP) which equals $28-29 \AA$ for HOPG and $\theta$ is the takeoff angle $\left(90^{\circ}\right.$ when the penetration depth is $8.7 \mathrm{~nm}$ and least surface sensitive). ${ }^{335,358,371}$ This yields a surface contribution $\left(\mathrm{I}_{\mathrm{S}}\right)$ of $6.0 \%$. Similar calculations can be made for PG and results are shown in Table 25.

The surface contribution $\left(I_{S}\right)$ for both graphite samples at $0^{\circ}$ is $4 \%$ and $6 \%$ depending on the calculation method. This means that the XPS data presented above (specifically Table 23) has only $c a .5 \%$ contribution from the surface layer. At $60^{\circ}$, there is $c a .50 \%$ difference between the two calculation methods: $I_{S}$ nearly doubles when the experiment becomes more surface 
sensitive yet almost 13 graphene layers are still probed. The sample would need to be tilted $85^{\circ}$ ( $5^{\circ}$ takeoff angle) for only 2 graphene layers to be probed; ${ }^{335}$ however, data could not be acquired above $60^{\circ}$ tilt for the instrument and set-up used.

Table 25. Contribution of surface layer to XPS spectrum.

\begin{tabular}{|c|c|c|c|c|}
\hline & \multicolumn{2}{|c|}{ ZYA } & \multicolumn{2}{c|}{ PG } \\
\hline $\mathrm{d}(\AA)$ & \multicolumn{2}{|c|}{3.36} & \multicolumn{2}{c|}{3.43} \\
\hline & $0^{\circ}$ & $60^{\circ}$ & $0^{\circ}$ & $60^{\circ}$ \\
\hline $\mathrm{N}_{\mathrm{GL}}$ & 25.9 & 12.8 & 25.4 & 12.5 \\
\hline $\mathrm{I}_{\mathrm{S}}$ (Equation 58) & $3.9 \%$ & $7.8 \%$ & $3.9 \%$ & $8.0 \%$ \\
\hline $\mathrm{I}_{\mathrm{S}}$ (Equation 59) & $6.0 \%$ & $12.3 \%$ & $6.1 \%$ & $12.6 \%$ \\
\hline
\end{tabular}

Note: See Appendix B for calculation of interlayer spacing (d). The penetration depth at $60^{\circ}$ ( $30^{\circ}$ takeoff angle) is $4.3 \mathrm{~nm}$.

Hydrocarbon contamination occurs at the surface and does not diffuse or intercalate below the uppermost graphene layer; therefore, contributions from contamination originate on the surface. Moreover, contamination is expected to be small since the sample is exposed to ambient air for $<30$ seconds before being placed in the XPS preparation chamber. Once in the chamber, however, pumping down to UHV takes 20-30 minutes, in which time contaminants can still adsorb onto the surface although kinetics is significantly restricted. There is likely submonolayer coverage of hydrocarbon contaminants on the graphitic surface which adsorb to high energy defect sites. 
C-C photoelectrons originate throughout the entire sample and the contribution of surface carbons are only $c a .8-12 \%$ even when the sample is tilted $60^{\circ}$. This means that $c a .90 \%$ of the signal originates from bulk electrons and makes elucidating the surface defect density extremely difficult. Future investigations should strongly consider testing at the highest angle possible where the sample is tilted $80^{\circ}\left(10^{\circ}\right.$ takeoff angle) as demonstrated by Smith et al..$^{358}$ and Speranza et al. ${ }^{335}$ This will significantly increase surface sensitivity and allow for the spectra to be fit using the Doniach-Sunjic asymmetry parameter for surface and bulk components. ${ }^{335,358,371}$

\section{C.5 XPS CONCLUSIONS}

XPS was used to elucidate the chemical nature of high and low quality graphite samples. Originally, we postulated that the defect density could be calculated by comparing the at $\%$ of photoelectrons originating from delocalized $\mathrm{sp}^{2}$-hybridized carbon; however, this proved unobtainable for several reasons illustrated above. Nevertheless, valuable information pertaining to the quality of graphite samples and its hybridization was obtained through XPS and AR-XPS analysis. Survey spectra on freshly exfoliated samples show that ZYA consists of $99.6 \%$ carbon ( $0.4 \%$ oxygen) and PG consists of $99.1 \%$ carbon $(0.9 \%$ oxygen $)$. High resolution $\mathrm{C} 1 \mathrm{~s}$ spectra

indicate the graphite chemistry is more nuanced: ZYA has $5 \%$ more $\mathrm{sp}^{2}$-hybridized carbon than PG.

C1s spectra show that the pristine $\mathrm{sp}^{2}$ carbon content of ZYA and PG is $79 \%$ and $74 \%$, respectively. Defect sites and adsorbed oxygen contribute to the remaining $21 \%$ and $26 \%$. Unequivocally, ZYA is a higher quality sample with less defects and more adsorbed oxygen. The latter phenomena may be a consequence of oxygen preferentially adsorbing to the basal 
plane. In conclusion, ZYA and PG are $\mathrm{sp}^{2}$-hybridized carbon $(\mathrm{C} 1$ peak centered at $284.3 \mathrm{eV})$ with few surface defects and a small amount of adsorbed oxygen on the surface. ZYA has a greater amount of pristine $\mathrm{sp}^{2}$ carbon and less defects.

While this XPS data is very informative and provides information on sample quality, it does not provide direct evidence of defect density. High resolution C1s scans do show the relative contribution of $\mathrm{sp}^{2}$-hybridized carbon to other adsorbed species; however, the data does not provide a quantitative value for the amount of defects present on the sample surface. The amount of defects can only be estimated by analyzing the amount of delocalized and non- $\mathrm{sp}^{2}$ hybridized carbon and assuming that this contaminant contribution is proportional to the amount of defects (i.e., defect density) on the sample surface. Although reasonable, this method is convoluted and does not provide direct quantitative defect data; therefore, Raman spectroscopy was used to determine defect density on graphite samples instead of XPS. 


\section{APPENDIX D}

\section{DETAILS OF XPS DATA ACQUISITION AND ANALYSIS}

\section{D.1 X-RAY SPOT SIZE: $200 \mu \mathrm{m} v s .900 \mu \mathrm{m}$}

The ESCALAB 250Xi allows for the x-ray size to be adjusted between $200 \mu \mathrm{m}$ and $900 \mu \mathrm{m}$. All XPS experiments presented in the main document were conducted at $200 \mu \mathrm{m}$; however, this parameter was decided by first testing ZYA at both spot sizes. Figure 38 shows C1s spectra at both $200 \mu \mathrm{m}$ and $900 \mu \mathrm{m}$ spot size. The shape of both spectra are similar. The $900 \mu \mathrm{m}$ spot size has more asymmetry towards higher binding energy indicating more defects. ${ }^{357,360,370}$ This makes sense because $4.5 \mathrm{x}$ more surface is being tested at $900 \mu \mathrm{m}$ compared to $200 \mu \mathrm{m}$, thus more defects are detected. The spectra were fit using the PsdVoigtl function and fitting results are shown in Table 26.

The most significant difference between the two spot sizes is the $\mathrm{C} 2$ and $\mathrm{C} 3$ at $\%$. $\mathrm{C} 2$ is greater at $900 \mu \mathrm{m}$ and C3 is greater at $200 \mu \mathrm{m}$. This could be due to the type of defects being detected. C2 is localized electrons due to defects and adsorbed oxygen. C3 is free radicals and $\mathrm{C}-\mathrm{O}-\mathrm{C}$ bonding. At smaller spot size the amount of free radicals and localized defects are similar. At larger spot size, more localized defects are detected indicating that free radical and 
C-O-C sites are less common. The overall amount of defects are similar: 15.1 at $\%$ for $200 \mu \mathrm{m}$ and 17.1 at $\%$ for $900 \mu \mathrm{m}$. Additionally, $\mathrm{C} 1$ at $\%$ is similar at both spot sizes. This indicates that $\mathrm{x}$-ray spot size does not have an impact on the drawn conclusions.

Table 26. XPS fitting results of ZYA C1s spectra for different $\mathrm{x}$-ray spot sizes. Data is presented as the average atomic\% (standard deviation).

\begin{tabular}{|c|c|c|}
\hline & $200 \mu \mathrm{m}$ & $900 \mu \mathrm{m}$ \\
\hline $\mathrm{C} 1$ & $\begin{array}{c}\mathbf{6 8 . 3} \\
\mathbf{( 7 . 4 )}\end{array}$ & $\begin{array}{c}\mathbf{6 6 . 6} \\
\mathbf{( 5 . 8 )}\end{array}$ \\
\hline $\mathrm{C} 2$ & $\begin{array}{c}7.7 \\
(0.8)\end{array}$ & $\begin{array}{c}14.3 \\
(2.9)\end{array}$ \\
\hline $\mathrm{C} 3$ & $\begin{array}{c}7.4 \\
(4.5)\end{array}$ & $\begin{array}{c}2.8 \\
(1.9)\end{array}$ \\
\hline $\mathrm{C} 4$ & $\begin{array}{c}2.3 \\
(2.4)\end{array}$ & $\begin{array}{c}1.3 \\
(1.1)\end{array}$ \\
\hline $\mathrm{C} 5$ & $\begin{array}{c}14.2 \\
(0.4)\end{array}$ & $\begin{array}{c}14.9 \\
(0.1)\end{array}$ \\
\hline $\mathrm{R}-\chi^{2}$ & $3.15 \mathrm{e}-5$ & $8.08 \mathrm{e}-5$ \\
\hline
\end{tabular}

\section{D.2 C1s FITTING PROCEDURE}

Fitting the C1s spectrum can be done either by a Doniach-Sunjic function which accounts for asymmetry towards higher binding energy or deconvoluting the peak into several asymmetric peaks. ${ }^{360,363}$ The Doniach-Sunjic approach provides information on the relative amount of 
defects (since asymmetry increases with defects) but not the type of defects. Deconvoluting into several peaks will provide more detailed information on the type of defects and their contribution to the overall spectra. Therefore, it was chosen to deconvolute the C1s spectra of ZYA and PG into five peaks. The five peaks were chosen according to data presented by Yang and Sacher. ${ }^{360}$, 363 The peak attribution and peak center $\left(\mathrm{x}_{\mathrm{c}}\right)$ are shown in Table 23.

Five peak fitting functions were selected based on data found in literature for fitting $\mathrm{C} 1 \mathrm{~s}$ spectra and functions available in OriginPro. Factors influencing the fit of each peak are: peak position $\left(\mathrm{x}_{\mathrm{c}}\right)$, area $(\mathrm{A})$, full width at half maximum (FWHM), and \% Gaussian-Lorentzian $(\mu)$. Table 27 and Table 28 show details of each function and their fitting parameters. The PsdVoigt functions are controlled by a profile shape factor $(\mu)$ which dictates weight of the Lorentzian and Gaussian terms. The peak is $100 \%$ Gaussian when $\mu=0$ and the peak is $100 \%$ Lorentzian when $\mu=1$. Therefore, $\mu=0.2$ means that the peak is $20 \%$ Lorentzian and $80 \%$ Gaussian. ${ }^{368}$ 

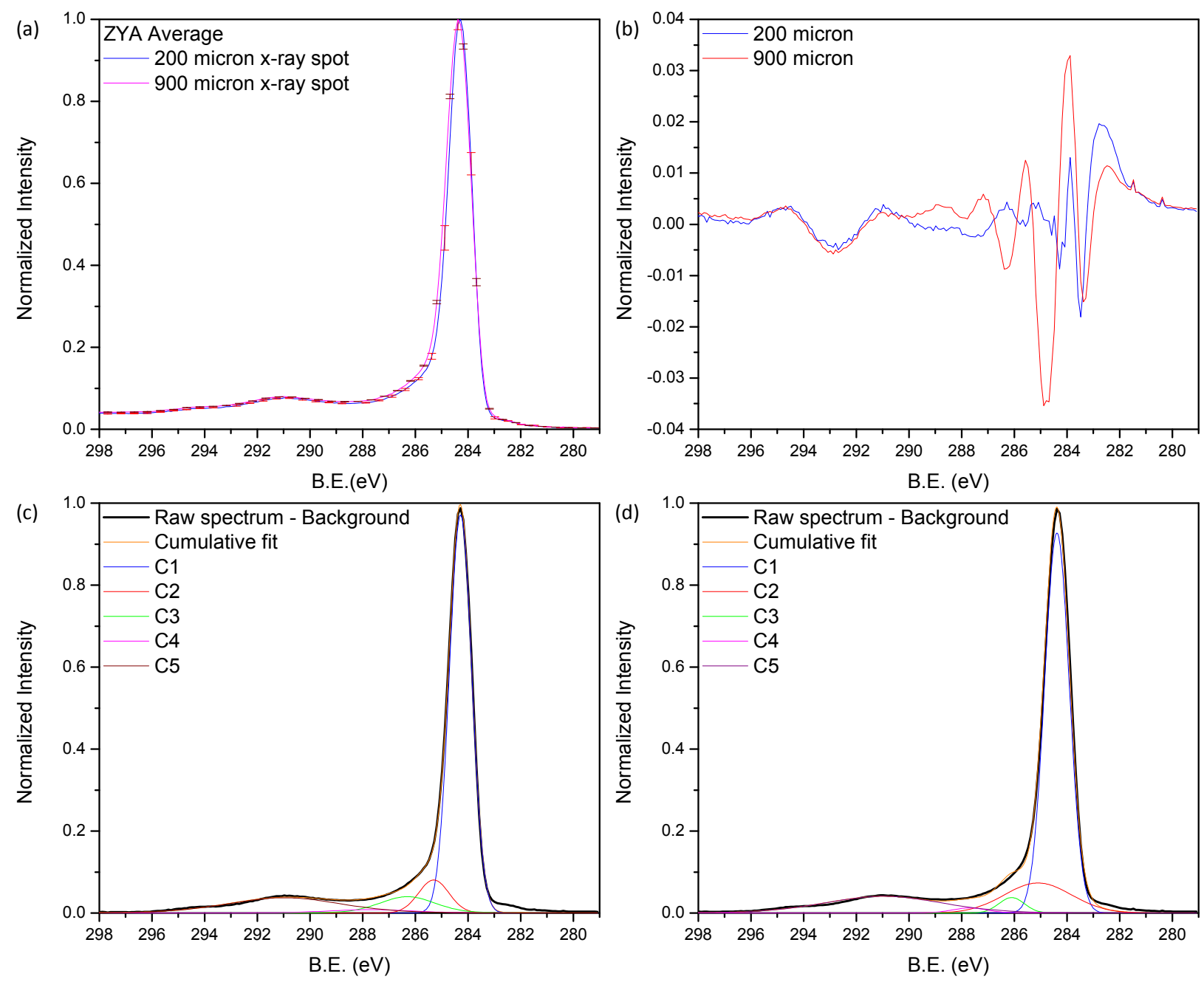

Figure 38. High resolution C1s spectra for different $\mathrm{x}$-ray spot sizes. (a) $200 \mu \mathrm{m}$ spectrum is the average of five separate experiments and $900 \mu \mathrm{m}$ spectrum is the average of three separate experiments. (b) Residual/Error of PsdVoigt1 model. (c) $200 \mu \mathrm{m}$ and (d) $900 \mu \mathrm{m}$ spectrum fit with five symmetric peaks. Shirley background was subtracted from raw data and peaks were fit with PsdVoigt 1 function with $\mu=0$ for $\mathrm{C} 1-\mathrm{C} 2$ and $\mu=0.2$ for C3-C5. 
Table 27. Details of functions used for fitting the $\mathrm{C} 1 \mathrm{~s}$ spectrum. ${ }^{368}$

\begin{tabular}{|c|c|}
\hline Function & $y=y_{0}+\frac{A e^{\frac{-4 \ln (2)\left(x-x_{c}\right)^{2}}{F W H M^{2}}}}{F W H M \sqrt{\frac{\pi}{4 \ln (2)}}}$ \\
\hline Gaussian & $y=y_{0}+\frac{A e^{\frac{-2\left(x-x_{c}\right)^{2}}{w^{2}}}}{w \sqrt{\frac{\pi}{2}}} \quad w=\frac{F W H M}{\sqrt{\ln 4}} \quad \sigma=\frac{w}{2}$ \\
\hline Gauss & $y=y_{0}+\frac{2 A}{\pi} \frac{F W H M}{4\left(x-x_{c}\right)^{2}+F W H M^{2}}$ \\
\hline Lorentzian & $y=y_{0}+A\left[\mu \frac{2}{\pi} \frac{F W H M}{4\left(x-x_{c}\right)^{2}+F W H M^{2}}\right]+(1-\mu) \frac{e^{\frac{-4 \ln (2)\left(x-x_{c}\right)^{2}}{F W H M^{2}}}}{F W H M \sqrt{\frac{\pi}{4 \ln (2)}}}$ \\
\hline PsdVoigt2 & $y=y_{0}+A\left[\mu \frac{2}{\pi} \frac{F W H M_{L}}{4\left(x-x_{c}\right)^{2}+F W H M_{L}^{2}}\right]+(1-\mu) \frac{e^{\frac{-4 \ln (2)\left(x-x_{c}\right)^{2}}{F W H M_{G}^{2}}}}{F W H M_{G} \sqrt{\frac{\pi}{4 \ln (2)}}}$ \\
\hline Doniach-Sunjic & $y=y_{0}+\frac{H \cos \left(\frac{a \pi}{2}+(1-a) \tan { }^{-1}\left(\frac{x-x_{c}}{w}\right)\right)}{\sqrt{\left(w^{2}+\left(x-x_{c}\right)^{2}\right)^{(1-a)}}}$ \\
\hline
\end{tabular}


Table 28. Parameters of functions used for fitting the C1s spectrum. ${ }^{368}$

\begin{tabular}{|c|c|c|c|c|}
\hline Function & Notes & Fit & Constant & Calculated \\
\hline Gaussian & FWHM version of Gaussian & $\begin{array}{c}\text { A } \\
\text { FWHM }\end{array}$ & $\begin{array}{l}\mathrm{y}_{\mathrm{o}} \\
\mathrm{x}_{\mathrm{c}}\end{array}$ & --- \\
\hline Gauss & Area version of Gaussian & $\begin{array}{l}\text { A } \\
\text { W }\end{array}$ & $\begin{array}{l}\mathrm{y}_{\mathrm{o}} \\
\mathrm{x}_{\mathrm{c}}\end{array}$ & $\begin{array}{c}\sigma \\
\text { FWHM } \\
\text { H }\end{array}$ \\
\hline Lorentzian & --- & $\begin{array}{c}\text { A } \\
\text { FWHM }\end{array}$ & $\begin{array}{l}\mathrm{y}_{\mathrm{o}} \\
\mathrm{x}_{\mathrm{c}}\end{array}$ & $\mathrm{H}$ \\
\hline PsdVoigt1 & $\begin{array}{l}\text { Linear combination of } \\
\text { Gaussian and Lorentzian } \\
\text { with same FWHM }\end{array}$ & $\begin{array}{c}\mathrm{A} \\
\mathrm{FWHM}\end{array}$ & $\begin{array}{l}\mathrm{y}_{\mathrm{o}} \\
\mathrm{x}_{\mathrm{c}} \\
\mu\end{array}$ & --- \\
\hline PsdVoigt2 & $\begin{array}{l}\text { Linear combination of } \\
\text { Gaussian and Lorentzian } \\
\text { with separate FWHM }\end{array}$ & $\begin{array}{c}\text { A } \\
\text { FWHM }_{\mathrm{G}} \\
\text { FWHM }_{\mathrm{L}}\end{array}$ & $\begin{array}{l}\mathrm{y}_{\mathrm{o}} \\
\mathrm{x}_{\mathrm{c}} \\
\mu\end{array}$ & --- \\
\hline Doniach-Sunjic & --- & $\begin{array}{l}\mathrm{H} \\
\mathrm{W} \\
\alpha\end{array}$ & $\begin{array}{l}\mathrm{y}_{\mathrm{o}} \\
\mathrm{x}_{\mathrm{c}}\end{array}$ & --- \\
\hline
\end{tabular}

Note: $\mathrm{A}=$ peak area; $\mathrm{w}=$ width; $\mathrm{FWHM}=$ full width at half maximum; $\mathrm{FWHM}_{\mathrm{L}}=\mathrm{FWHM}$ of Lorentzian term; $\mathrm{FWHM}_{\mathrm{G}}=\mathrm{FWHM}$ of Gaussian term; $\mathrm{H}=$ peak height; $\mathrm{y}_{\mathrm{o}}=\mathrm{y}$-offset $=0$; $\mathrm{x}_{\mathrm{c}}=$ peak center; $\mu=\%$ Gaussian-Lorentzian; $\alpha=$ asymmetry parameter.

Several combinations of the functions in Table 27 were used to determine which resulted in the best fit. Figure 40 shows some examples of spectra fit by various models and Figure 39 shows quantitative fitting data. Lorentzian and PsdVoigt2 fits were always the worst and removed from consideration. This left Gaussian, Gauss, and PsdVoigt1. Previous researchers have commonly settled on a mixture of Gaussian and Lorentzian curves, ${ }^{357,358,363,364}$ so several combinations were tried. Ultimately, PsdVoigt1 resulted in the best fitting with the lowest reduced $-\chi^{2}$. To determine the best $\% \mathrm{G}-\mathrm{L}(\mu)$, the peaks were fit by (a) $\mu=0.2(80 \%$ Gaussian) as shown in Figure 40c and (b) $\mu=0$ (100\% Gaussian) for C1-C2 and $\mu=0.2(20 \%$ Lorentzian) for 
C3-C5 as shown in Figure 40d. The second scheme (b) was chosen because it included a Lorentzian term for peaks C3-C5, which is supported by previous work. ${ }^{357,358,363,364}$ Table 29 includes notes on each model. Conclusion: The PsdVoigt1 model was chosen where $\mu=0$ for C1$\mathrm{C} 2$ and $\mu=0.2$ for $\mathrm{C} 3-\mathrm{C} 5$.

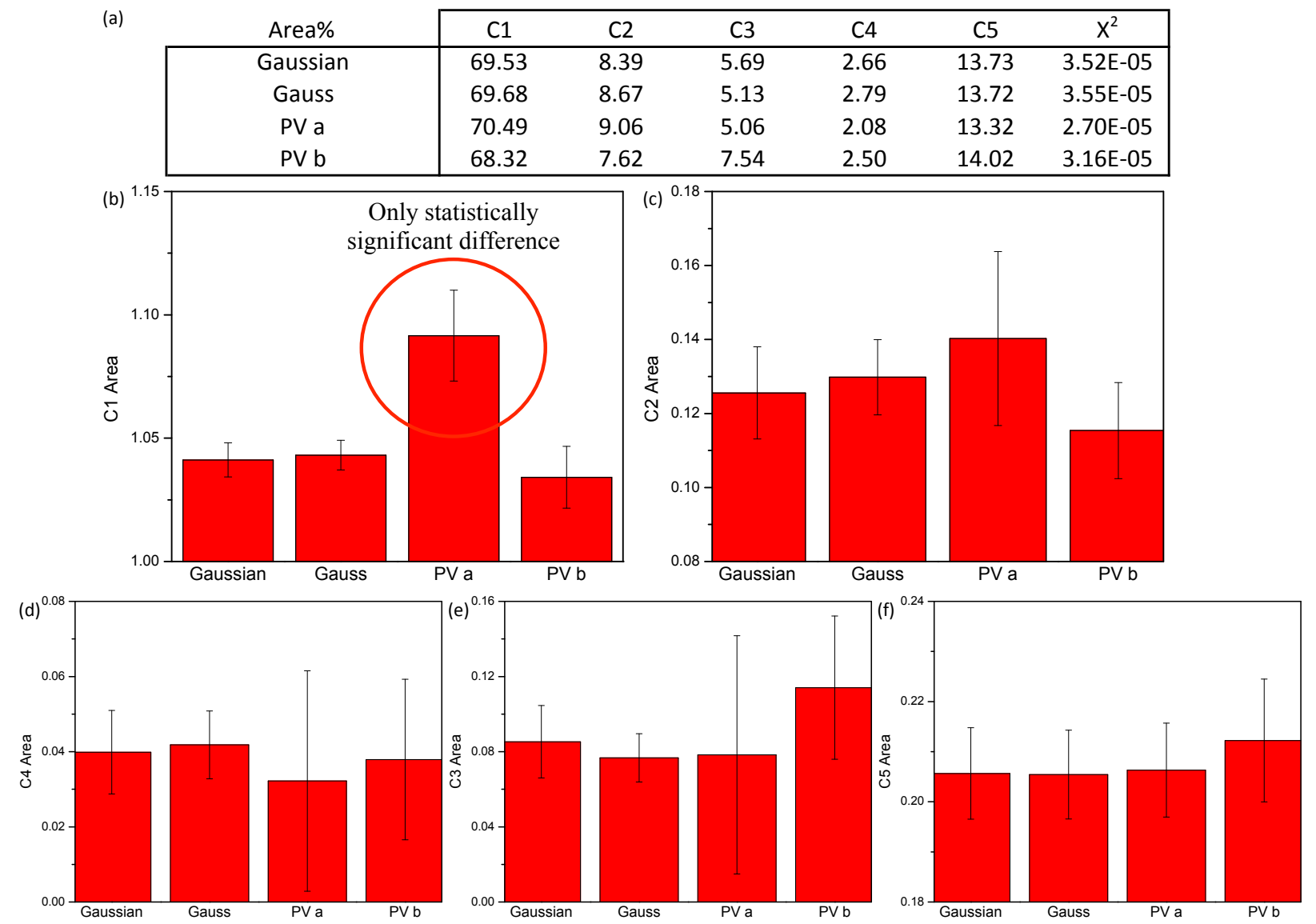

Figure 39. Fitting results of C1s spectra by various models. (a) Area\% of peaks C1-C5 fit by Gaussian, Gauss, and PsdVoigt functions and reduced- $\chi^{2}$ for models. (b-f) Calculated area for the modeled spectra. Gaussian: All peaks are fit by Gaussian function. Gauss: All peaks are fit by Gauss function. PVa: PsdVoigt1 function where $\mu=0.20$ for all peaks. PVb: PsdVoigt1 function where $\mu=0$ for $\mathrm{C} 1-\mathrm{C} 2$ and $\mu=0.2$ for $\mathrm{C} 3-\mathrm{C} 5$. 

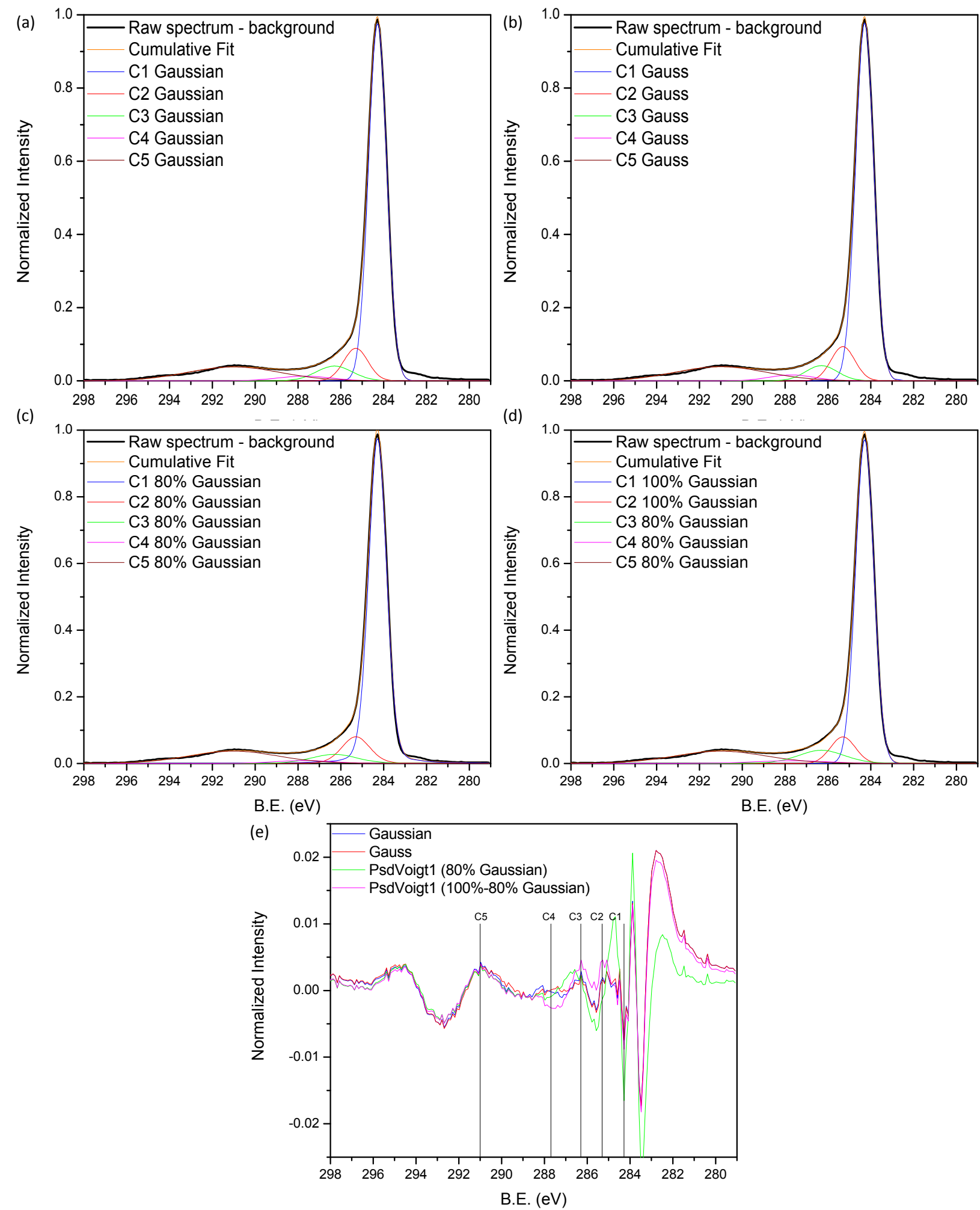

Figure 40. Peak fitting the $\mathrm{C} 1 \mathrm{~s}$ spectra using various models. Spectrum fit using (a) the Gaussian function, (b) the Gauss function, (c) $80 \%$ Gaussian function, (d) mixed 100\%/80\% Gaussian function. (e) Residual/Error of models. 


\section{D.3 COMMENTS ON ERROR OF C1s FIT}

The C1s fitting data presented in Figure 35 and Table 23 clearly show differences in the chemical composition of ZYA and PG. ZYA has a greater amount of pristine $\mathrm{sp}^{2}$ carbon (C1); however, this difference is reduced from 6.5 at $\%$ to 1.6 at $\%$ when error is considered. Even at the limit of experimental error, ZYA exhibits greater pristine surface than PG. Additionally, C2 at $\%$ is greater on PG than ZYA by $1 \%$ when considering error. There is no difference in the C3 peak of the two samples.

Peak fitting is an iterative process which converges when the error between the raw data and model is at a minimum. The error is quantitatively depicted as reduced $\chi^{2}$ which was $3.23 \mathrm{e}-$ 5 and 4.27e-5 for ZYA and PG, respectively, and the $\mathrm{R}^{2}$ value was 0.999 for both samples. While the overall fitting is very good, the fitting of each peak (C1-C5) has some degree of associated variability because the fitting is dependent on the other peaks within the model. In other words, $\mathrm{C} 1$ is dependent on $\mathrm{C} 2-\mathrm{C} 5$ and so forth. This results in intrinsic error of the model itself which is realistically estimated to be $1-3 \%$ (Figure $35 b$ ). This error is included in the standard deviation shown in Table 23.

To conclude, error has an effect on the quantitative conclusions pertaining to the defects of ZYA and PG. The data clearly shows that ZYA has greater amount of pristine carbon and less amount of defects. This qualitative conclusion remains true when error is considered, even though the quantitative values change. 


\section{D.4 DONIACH-SUNJIC C1s FIT}

Figure 41 shows the C1s fitting result for ZYA and PG using the Doniach-Sunjic function for peaks $\mathrm{C} 1-\mathrm{C} 3$ and the PsdVoigt1 function $(\mu=0.2)$ for peaks C4-C5. This provided a better fit than using the Doniach-Sunjic function for C1-C2 and the PsdVoigt1 function for C3-C5. Either way, the asymmetry parameter $(\alpha)$ was $c a .0 .07$ for ZYA and 0.08 for PG, suggesting that intrinsic defects can not be quantified by the Doniach-Sunjic function. Intentional defects, however, caused by irradiating with ions can be quantified by this function. ${ }^{360}$

Table 29. Observations and notes of peak fitting models.

\begin{tabular}{|c|c|}
\hline Function & Notes \\
\hline Gaussian & $\begin{array}{l}\text { - All peaks able to be fit. } \\
\text { - Similar result to other models. } \\
\text { - Lower } \chi^{2} \text { than Gauss. }\end{array}$ \\
\hline Gauss & $\begin{array}{l}\text { - All peaks able to be fit. } \\
\text { - Similar results to other models. } \\
\text { - Same result as Gaussian with slightly higher } \chi^{2} \text {. }\end{array}$ \\
\hline Lorentzian & $\begin{array}{l}\text { - Always fails to fit C4. Peak area becomes negative. } \\
\text { - Higher } \chi^{2} \text { than other models. }\end{array}$ \\
\hline PsdVoigt1 & $\begin{array}{l}\text { - All peaks able to be fit with result similar to Gaussian and Gauss. } \\
\text { - PVa: Fit all peaks with } \mu=0.2(80 \% \text { Gaussian). } \\
\text { - PVb: Fit } \mathrm{C} 1-\mathrm{C} 2 \text { with } \mu=0 \text { ( } 100 \% \text { Gaussian) and C3-C } 5 \text { with } \mu=0.2(80 \% \text { Gaussian }) \\
\text { - Allowing } \mu \text { to be fit (opposed to constant) does not provide realistic results. }\end{array}$ \\
\hline PsdVoigt2 & - Always fails to fit $\mathrm{C} 2$. $\mathrm{FWHM}_{\mathrm{L}}$ is unrealistic for $\mathrm{C} 2$ and $\mathrm{C} 4$. \\
\hline
\end{tabular}



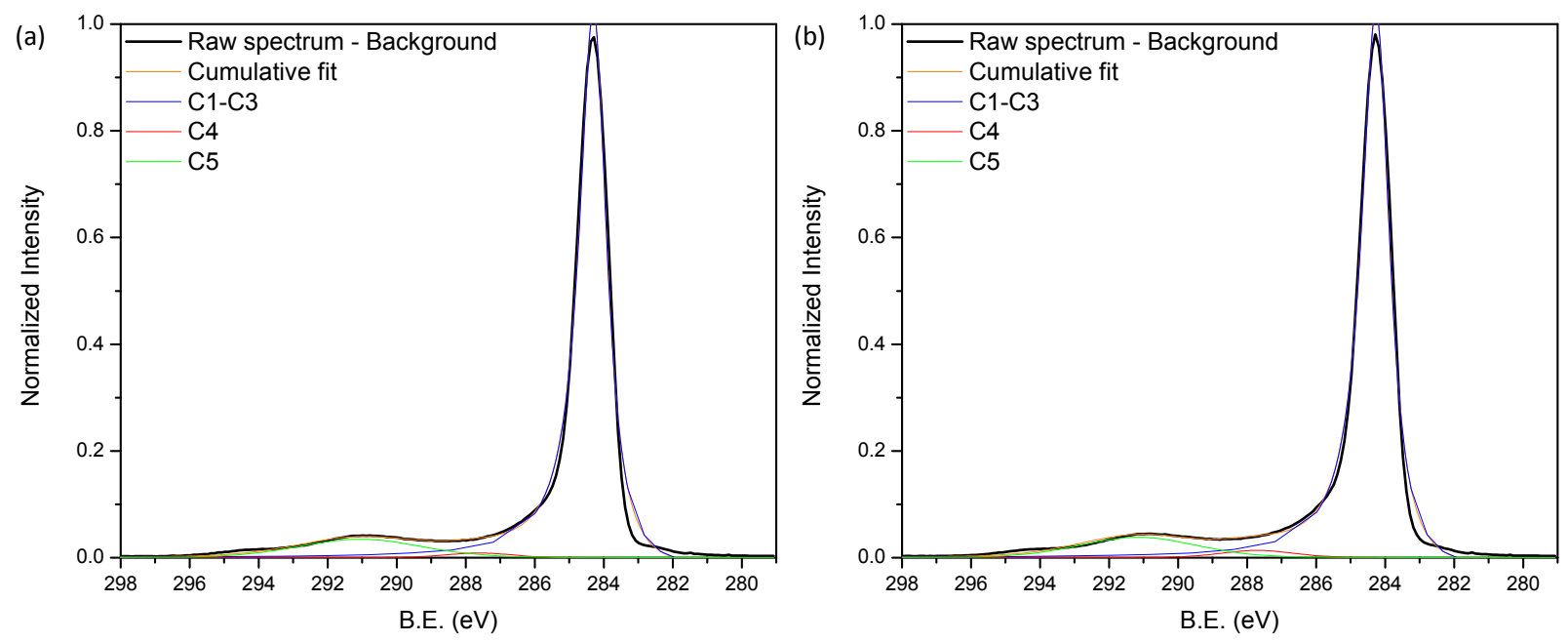

Figure 41. Doniach-Sunjic fitting results of the $\mathrm{C} 1$ s spectrum. C1-C3 were fit using the Doniach-Sunjic function and C4-C5 were fit using the PsdVoigt $(\mu=0.2)$ function for (a) ZYA and (b) PG. 


\section{BIBLIOGRAPHY}

1. Spreadborough, J. The Frictional Behaviour of Graphite. Wear 1962, 5, 18-30.

2. Ohler, M.; Baruchel, J.; Galez, P. An X-Ray Diffraction Topographic Study of Highly Oriented Pyrolytic Graphite. J. Phys. D: Appl. Phys. 1995, 28, A78.

3. Erlich, E. I.; Dan Hausel, W. Diamond Deposits: Origin, Exploration, and History of Discovery; Society for Mining, Metallurgy, and Exploration: 2002; pp 74-94.

4. Erdosh, G. Geology of Bogala Mine, Ceylon and the Origin of Vein-Type Graphite. Miner. Deposita 1970, 5, 375-382.

5. Liu, S.; Loper Jr, C. R. The Formation of Kish Graphite. Carbon 1991, 29, 547-555.

6. Nelson, J. B.; Riley, D. P. The Thermal Expansion of Graphite from $15^{\circ} \mathrm{C}$ to $800^{\circ} \mathrm{C}$ : Part I. Experimental. Pro. Phy. Soc. 1945, 57, 477.

7. Tuffanelli, A.; Sanchez del Rio, M.; Pareschi, G.; Gambaccini, M.; Taibi, A.; Fantini, A.; Ohler, M. Comparative Characterization of Highly Oriented Pyrolytic Graphite by Means of Diffraction Topography. Proc. SPIE 1999, 3773, 192-198.

8. Blackman, L. C. F.; Ubbelhode, A. R. Stress Recrystallization of Graphite. Proc. R. Soc. A 1962, 266, 20-32.

9. Bacon, G. The Interlayer Spacing of Graphite. Acta Crystallogr. 1951, 4, 558-561.

10. Walker, P. L.; McKinstry, H. A.; Wright, C. C. X-Ray Diffraction Studies of a Graphitized Carbon - Changes in Interlayer Spacing and Binding Energy with Temperature. Ind. Eng. Chem. Res. 1953, 45, 1711-1715.

11. Li, Z. Q.; Lu, C. J.; Xia, Z. P.; Zhou, Y.; Luo, Z. X-Ray Diffraction Patterns of Graphite and Turbostratic Carbon. Carbon 2007, 45, 1686-1695.

12. Novoselov, K. S.; Geim, A. K.; Morozov, S. V.; Jiang, D.; Zhang, Y.; Dubonos, S. V.; Grigorieva, I. V.; Firsov, A. A. Electric Field Effect in Atomically Thin Carbon Films. Science 2004, 306, 666-669. 
13. Lee, C.; Wei, X.; Kysar, J. W.; Hone, J. Measurement of the Elastic Properties and Intrinsic Strength of Monolayer Graphene. Science 2008, 321, 385-388.

14. Darwin, C. G. XCII. The Reflexion of X-Rays from Imperfect Crystals. Philos. Mag. S. 6 1922, 43, 800-829.

15. Thomson, G. P. Charles Galton Darwin. 1887-1962. Biogr. Mem. Fellows R. Soc. 1963, 9, 69-85.

16. Charles Galton Darwin.

https://en.wikipedia.org/w/index.php?title=Charles_Galton_Darwin\&oldid=703119754 (accessed February 9, 2016).

17. SPI Supplies HOPG. http://www.2spi.com/category/hopg-spi-supplies/hopg/ (accessed February 9, 2016).

18. HOPG Highly Ordered Pyrolytic Graphic [sic]. http://www.2spi.com/category/hopg/ (accessed February 12, 2016).

19. Mao, Y.; Chen, C.-L.; Zhang, Y. Molecular Dynamic Study on Contact Angle of Water Droplet on a Single-Wall Carbon Nanotube (SWCNT) Plate. Appl. Phys. A 2013, 111, 747-754.

20. Lau, K. K. S.; Bico, J.; Teo, K. B. K.; Chhowalla, M.; Amaratunga, G. A. J.; Milne, W. I.; McKinley, G. H.; Gleason, K. K. Superhydrophobic Carbon Nanotube Forests. Nano Lett. 2003, 3, 1701-1705.

21. Fowkes, F. M.; Harkins, W. D. The State of Monolayers Adsorbed at the Interface Solid-Aqueous Solution. J. Am. Chem. Soc. 1940, 62, 3377-3386.

22. Taherian, F.; Marcon, V.; van der Vegt, N. F. A.; Leroy, F. What Is the Contact Angle of Water on Graphene? Langmuir 2013, 29, 1457-1465.

23. Wang, S.; Zhang, Y.; Abidi, N.; Cabrales, L. Wettability and Surface Free Energy of Graphene Films. Langmuir 2009, 25, 11078-11081.

24. Morcos, I. On Contact Angle and Dispersion Energy of the Cleavage Graphite/Water System. J. Colloid Interface Sci. 1970, 34, 469-471.

25. Morcos, I. Surface Tension of Stress-Annealed Pyrolytic Graphite. J. Chem. Phys. 1972, 57, 1801-1802.

26. Ou, J.; Wang, J.; Liu, S.; Mu, B.; Ren, J.; Wang, H.; Yang, S. Tribology Study of Reduced Graphene Oxide Sheets on Silicon Substrate Synthesized via Covalent Assembly. Langmuir 2010, 26, 15830-15836.

27. Raj, R.; Maroo, S. C.; Wang, E. N. Wettability of Graphene. Nano Lett. 2013, 13, 15091515. 
28. Shin, Y. J.; Wang, Y.; Huang, H.; Kalon, G.; Wee, A. T. S.; Shen, Z.; Bhatia, C. S.; Yang, H. Surface-Energy Engineering of Graphene. Langmuir 2010, 26, 3798-3802.

29. Adamson, A. W.; Gast, A. P. The Solid-Liquid Interface- Contact Ange: Experimental Methods and Measurements of Contact Angle. In Physical Chemistry of Surfaces; John Wiley \& Sons, Inc.: New York, 1997; pp 362-372.

30. Kogan, M. J.; Dalcol, I.; Gorostiza, P.; Lopez-Iglesias, C.; Pons, R.; Pons, M.; Sanz, F.; Giralt, E. Supramolecular Properties of the Proline-Rich $\Gamma$-Zein N-Terminal Domain. Biophys. J. 2002, 83, 1194-1204.

31. Westreich, P.; Fortier, H.; Flynn, S.; Foster, S.; Dahn, J. R. Exclusion of Salt Solutions from Activated Carbon Pores and the Relationship to Contact Angle on Graphite. J. Phys. Chem. C 2007, 111, 3680-3684.

32. Tadros, M. E.; Hu, P.; Adamson, A. W. Adsorption and Contact Angle Studies: I. Water on Smooth Carbon, Linear Polyethylene, and Stearic Acid-Coated Copper. J. Colloid Interface Sci. 1974, 49, 184-195.

33. Schrader, M. E. Ultrahigh Vacuum Techniques in the Measurement of Contact Angles. IV. Water on Graphite (0001). J. Phys. Chem. 1975, 79, 2508-2515.

34. Schrader, M. E. Ultrahigh-Vacuum Techniques in the Measurement of Contact Angles. 5. LEED Study of the Effect of Structure on the Wettability of Graphite. J. Phys. Chem. 1980, 84, 2774-2779.

35. Daniel, S. G. The Adsorption on Metal Surfaces of Long Chain Polar Compounds from Hydrocarbon Solutions. T. Faraday Soc. 1951, 47, 1345-1359.

36. Liu, P.; Cao, L.; Zhao, W.; Xia, Y.; Huang, W.; Li, Z. Insights into the Superhydrophobicity of Metallic Surfaces Prepared by Electrodeposition Involving Spontaneous Adsorption of Airborne Hydrocarbons. Appl. Surf. Sci. 2015, 324, 576-583.

37. Russell, A. M.; Lee, K. L. Processing Methods: Surface Modification. In StructureProperty Relations in Nonferrous Metals; John Wiley \& Sons: Hoboken, NJ, 2005; pp 127.

38. Gaines Jr, G. L. On the Water Wettability of Gold. J. Colloid Interface Sci. 1981, 79, 295.

39. Schneegans, M.; Menzel, E. Gold Crystals Solidified in Air Are Hydrophilic. J. Colloid Interface Sci. 1982, 88, 97-99.

40. Smith, T. The Hydrophilic Nature of a Clean Gold Surface. J. Colloid Interface Sci. 1980, 75, 51-55. 
41. Shi, G.; Shen, Y.; Liu, J.; Wang, C.; Wang, Y.; Song, B.; Hu, J.; Fang, H. MolecularScale Hydrophilicity Induced by Solute: Molecular-Thick Charged Pancakes of Aqueous Salt Solution on Hydrophobic Carbon-Based Surfaces. Sci. Rep. 2014, 4, 6793.

42. Banks, C. E.; Davies, T. J.; Wildgoose, G. G.; Compton, R. G. Electrocatalysis at Graphite and Carbon Nanotube Modified Electrodes: Edge-Plane Sites and Tube Ends Are the Reactive Sites. Chem. Commun. 2005, 829-841.

43. Bowling, R. J.; Packard, R. T.; McCreery, R. L. Activation of Highly Ordered Pyrolytic Graphite for Heterogeneous Electron Transfer: Relationship between Electrochemical Performance and Carbon Microstructure. J. Am. Chem. Soc. 1989, 111, 1217-1223.

44. Cline, K. K.; McDermott, M. T.; McCreery, R. L. Anomalously Slow Electron Transfer at Ordered Graphite Electrodes: Influence of Electronic Factors and Reactive Sites. $J$. Phys. Chem. 1994, 98, 5314-5319.

45. Kneten, K. R.; McCreery, R. L. Effects of Redox System Structure on Electron-Transfer Kinetics at Ordered Graphite and Glassy Carbon Electrodes. Anal. Chem. 1992, 64, 25182524.

46. McDermott, M. T.; Kneten, K.; McCreery, R. L. Anthraquinonedisulfonate Adsorption, Electron-Transfer Kinetics, and Capacitance on Ordered Graphite Electrodes: The Important Role of Surface Defects. J. Phys. Chem. 1992, 96, 3124-3130.

47. McDermott, M. T.; McCreery, R. L. Scanning Tunneling Microscopy of Ordered Graphite and Glassy Carbon Surfaces: Electronic Control of Quinone Adsorption. Langmuir 1994, 10, 4307-4314.

48. McCreery, R. L.; McDermott, M. T. Comment on Electrochemical Kinetics at Ordered Graphite Electrodes. Anal. Chem. 2012, 84, 2602-2605.

49. Patel, A. N. Electroanalytical Applications of Carbon Electrodes. Doctoral Dissertation, University of Warwick, 2012. http://wrap.warwick.ac.uk/56386/

50. Patel, A. N.; Collignon, M. G.; O’Connell, M. A.; Hung, W. O. Y.; McKelvey, K.; Macpherson, J. V.; Unwin, P. R. A New View of Electrochemistry at Highly Oriented Pyrolytic Graphite. J. Am. Chem. Soc. 2012, 134, 20117-20130.

51. Patel, A. N.; McKelvey, K.; Unwin, P. R. Nanoscale Electrochemical Patterning Reveals the Active Sites for Catechol Oxidation at Graphite Surfaces. J. Am. Chem. Soc. 2012, 134, 20246-9.

52. Patel, A. N.; Tan, S. Y.; Unwin, P. R. Epinephrine Electro-Oxidation Highlights Fast Electrochemistry at the Graphite Basal Surface. Chem. Commun. 2013, 49, 8776-8.

53. Güell, A. G.; Cuharuc, A. S.; Kim, Y.-R.; Zhang, G.; Tan, S.-y.; Ebejer, N.; Unwin, P. R. Redox-Dependent Spatially Resolved Electrochemistry at Graphene and Graphite Step Edges. ACS Nano 2015, 9, 3558-3571. 
54. Unwin, P. R. Concluding Remarks: There's Nowt So Queer as Carbon Electrodes. Faraday Discuss. 2014, 172, 521-32.

55. Patel, A. N.; Tan, S.-y.; Miller, T. S.; Macpherson, J. V.; Unwin, P. R. Comparison and Reappraisal of Carbon Electrodes for the Voltammetric Detection of Dopamine. Anal. Chem. 2013, 85, 11755-11764.

56. Velický, M.; Bradley, D. F.; Cooper, A. J.; Hill, E. W.; Kinloch, I. A.; Mishchenko, A.; Novoselov, K. S.; Patten, H. V.; Toth, P. S.; Valota, A. T.; Worrall, S. D.; Dryfe, R. A. W. Electron Transfer Kinetics on Mono- and Multilayer Graphene. ACS Nano 2014, 8, 10089-10100.

57. Velický, M.; Bissett, M. A.; Toth, P. S.; Patten, H. V.; Worrall, S. D.; Rodgers, A. N. J.; Hill, E. W.; Kinloch, I. A.; Novoselov, K. S.; Georgiou, T.; Britnell, L.; Dryfe, R. A. W. Electron Transfer Kinetics on Natural Crystals of $\mathrm{MoS}_{2}$ and Graphite. Phys. Chem. Chem. Phys. 2015, 17, 17844-17853.

58. Patten, H. V.; Velický, M.; Clark, N.; Muryn, C. A.; Kinloch, I. A.; Dryfe, R. A. W. Electrochemistry of Well-Defined Graphene Samples: Role of Contaminants. Faraday Discuss. 2014, 172, 261-272.

59. Nioradze, N.; Chen, R.; Kurapati, N.; Khvataeva-Domanov, A.; Mabic, S.; Amemiya, S. Organic Contamination of Highly Oriented Pyrolytic Graphite as Studied by Scanning Electrochemical Microscopy. Anal. Chem. 2015, 87, 4836-4843.

60. Nioradze, N. Nanogap-Enabled Study of Electrode Reactions by Scanning Electrochemical Microscopy. Doctoral Dissertation, University of Pittsburgh, 2014. http://d-scholarship.pitt.edu/23890/

61. Chen, R.; Nioradze, N.; Santhosh, P.; Li, Z.; Surwade, S. P.; Shenoy, G. J.; Parobek, D. G.; Kim, M. A.; Liu, H.; Amemiya, S. Ultrafast Electron Transfer Kinetics of Graphene Grown by Chemical Vapor Deposition. Angew. Chem. Int. Ed. 2015, 54, 15134-15137.

62. Li, Z.; Kozbial, A.; Nioradze, N.; Parobek, D.; Shenoy, G. J.; Salim, M.; Amemiya, S.; Li, L.; Liu, H. Water Protects Graphitic Surface from Airborne Hydrocarbon Contamination. ACS Nano 2016, 10, 349-359.

63. Lai, C.-Y.; Tang, T.-C.; Amadei, C. A.; Marsden, A. J.; Verdaguer, A.; Wilson, N.; Chiesa, M. A Nanoscopic Approach to Studying Evolution in Graphene Wettability. Carbon 2014, 80, 784-792.

64. Amadei, C. A.; Lai, C.-Y.; Heskes, D.; Chiesa, M. Time Dependent Wettability of Graphite Upon Ambient Exposure: The Role of Water Adsorption. J. Chem. Phys. 2014, 141, 084709.

65. Boinovich, L. B.; Emelyanenko, A. M.; Pashinin, A. S.; Lee, C. H.; Drelich, J.; Yap, Y. K. Origins of Thermodynamically Stable Superhydrophobicity of Boron Nitride Nanotubes Coatings. Langmuir 2012, 28, 1206-1216. 
66. Wu, Y.; Aluru, N. R. Graphitic Carbon-Water Nonbonded Interaction Parameters. $J$. Phys. Chem. B 2013, 117, 8802-8813.

67. Accordino, S. R.; Montes de Oca, J. M.; Rodriguez Fris, J. A.; Appignanesi, G. A. Hydrophilic Behavior of Graphene and Graphene-Based Materials. J. Chem. Phys. 2015, $143,154704$.

68. Ramos-Alvarado, B.; Kumar, S.; Peterson, G. P. Wettability of Graphitic-Carbon and Silicon Surfaces: MD Modeling and Theoretical Analysis. J. Chem. Phys. 2015, 143, 044703.

69. Leroy, F.; Liu, S.; Zhang, J. Parametrizing Nonbonded Interactions from Wetting Experiments via the Work of Adhesion: Example of Water on Graphene Surfaces. $J$. Phys. Chem. C 2015, 119, 28470-28481.

70. Kozbial, A.; Gong, X.; Liu, H.; Li, L. Understanding the Intrinsic Water Wettability of Molybdenum Disulfide $\left(\mathrm{MoS}_{2}\right)$. Langmuir 2015, 31, 8429-8435.

71. Preston, D. J.; Miljkovic, N.; Sack, J.; Enright, R.; Queeney, J.; Wang, E. N. Effect of Hydrocarbon Adsorption on the Wettability of Rare Earth Oxide Ceramics. Appl. Phys. Lett. 2014, 105, 011601.

72. Zhang, W.; Ye, C.; Bi, L.; Yang, Z.; Zhou, R. Wetting and Diffusion of Water on Pristine and Strained Phosphorene. eprint arXiv:1512.02116 2015, 8.

73. Azimi, G.; Dhiman, R.; Kwon, H.-M.; Paxson, A. T.; Varanasi, K. K. Hydrophobicity of Rare-Earth Oxide Ceramics. Nature Mater. 2013, 12, 315-320.

74. Tian, Y.; Jiang, L. Wetting: Intrinsically Robust Hydrophobicity. Nature Mater. 2013, $12,291-292$.

75. Kozbial, A.; Li, Z.; Conaway, C.; McGinley, R.; Dhingra, S.; Vahdat, V.; Zhou, F.; D'Urso, B.; Liu, H.; Li, L. Study on the Surface Energy of Graphene by Contact Angle Measurements. Langmuir 2014, 30, 8598-8606.

76. Kozbial, A.; Li, Z.; Sun, J.; Gong, X.; Zhou, F.; Wang, Y.; Xu, H.; Liu, H.; Li, L. Understanding the Intrinsic Water Wettability of Graphite. Carbon 2014, 74, 218-225.

77. Li, Z.; Wang, Y.; Kozbial, A.; Shenoy, G.; Zhou, F.; McGinley, R.; Ireland, P.; Morganstein, B.; Kunkel, A.; Surwade, S. P.; Li, L.; Liu, H. Effect of Airborne Contaminants on the Wettability of Supported Graphene and Graphite. Nature Mater. 2013, 12, 925-931.

78. Abrams, L.; Cicha, W. V.; Manzer, L. E.; Subramoney, S. A New Catalyst for an Old Process Driven by Environmental Issues. In Stud. Surf. Sci. Catal., Corma, A.; Mendioroz, F. V. M. S.; Luis, J.; Fierro, G., Eds. Elsevier: 2000; Vol. 130, pp 455-460. 
79. Rodríguez-reinoso, F. The Role of Carbon Materials in Heterogeneous Catalysis. Carbon 1998, 36, 159-175.

80. Ammann, M.; Kalbarer, M.; Jost, D. T.; Tobler, L.; Rossler, E.; Piguet, D.; Gaggeler, H. W.; Baltensperger, U. Heterogeneous Production of Nitrous Acid on Soot in Polluted Air Masses. Nature 1998, 395, 157-160.

81. Korpiel, J. A.; Vidic, R. D. Effect of Sulfur Impregnation Method on Activated Carbon Uptake of Gas-Phase Mercury. Environ. Sci. Technol. 1997, 31, 2319-2325.

82. Ravishankara, A. R. Heterogeneous and Multiphase Chemistry in the Troposphere. Science 1997, 276, 1058-1065.

83. Hutter, J. L.; Bechhoefer, J. Calibration of Atomic-Force Microscope Tips. Rev. Sci. Instrum. 1993, 64, 1868-1873.

84. Ritzert, N. L.; Rodríguez-López, J.; Tan, C.; Abruña, H. D. Kinetics of Interfacial Electron Transfer at Single-Layer Graphene Electrodes in Aqueous and Nonaqueous Solutions. Langmuir 2013, 29, 1683-1694.

85. Giraudet, J.; Claves, D.; Guérin, K.; Dubois, M.; Houdayer, A.; Masin, F.; Hamwi, A. Magnesium Batteries: Towards a First Use of Graphite Fluorides. J. Power Sources 2007, $173,592-598$.

86. Lin, M.-C.; Gong, M.; Lu, B.; Wu, Y.; Wang, D.-Y.; Guan, M.; Angell, M.; Chen, C.; Yang, J.; Hwang, B.-J.; Dai, H. An Ultrafast Rechargeable Aluminium-Ion Battery. Nature 2015, 520, 324-328.

87. Zhao, M.-Q.; Zhang, Q.; Huang, J.-Q.; Tian, G.-L.; Nie, J.-Q.; Peng, H.-J.; Wei, F. Unstacked Double-Layer Templated Graphene for High-Rate Lithium-Sulphur Batteries. Nat. Commun. 2014, 5.

88. Huang, C.; Xiao, J.; Shao, Y.; Zheng, J.; Bennett, W. D.; Lu, D.; Saraf, L. V.; Engelhard, M.; Ji, L.; Zhang, J.; Li, X.; Graff, G. L.; Liu, J. Manipulating Surface Reactions in Lithium-Sulphur Batteries Using Hybrid Anode Structures. Nat. Commun. 2014, 5.

89. Berger, C.; Song, Z.; Li, X.; Wu, X.; Brown, N.; Naud, C.; Mayou, D.; Li, T.; Hass, J.; Marchenkov, A. N.; Conrad, E. H.; First, P. N.; Heer, W. A. d. Electronic Confinement and Coherence in Patterned Epitaxial Graphene. Science 2006, 312, 1191-1196.

90. Ahn, J.-H.; Choi, J.-Y.; Hong, B. H.; Jang, H.; Kim, J. M.; Kim, K. S.; Kim, K. S.; Kim, P.; Lee, S. Y.; Zhao, Y. Large-Scale Pattern Growth of Graphene Films for Stretchable Transparent Electrodes. Nature 2009, 457, 706-710.

91. Feller, D.; Jordan, K. D. Estimating the Strength of the Water/Single-Layer Graphite Interaction. J. Phys. Chem. A 2000, 104, 9971-9975. 
92. Garcia, R.; Osborne, K.; Subashi, E. Validity of the "Sharp-Kink Approximation" for Water and Other Fluids. J. Phys. Chem. B 2008, 112, 8114-8119.

93. Kota, A. K.; Kwon, G.; Choi, W.; Mabry, J. M.; Tuteja, A. Hygro-Responsive Membranes for Effective Oil-Water Separation. Nat. Commun. 2012, 3, 1025.

94. Park, H.; Rowehl, J. A.; Kim, K. K.; Bulovic, V.; Kong, J. Doped Graphene Electrodes for Organic Solar Cells. Nanotechnology 2010, 21, 505204-505209.

95. Lee, C.; Li, Q.; Kalb, W.; Liu, X.-Z.; Berger, H.; Carpick, R. W.; Hone, J. Frictional Characteristics of Atomically Thin Sheets. Science 2010, 328, 76-80.

96. Mohanty, N.; Berry, V. Graphene-Based Single-Bacterium Resolution Biodevice and DNA Transistor: Interfacing Graphene Derivatives with Nanoscale and Microscale Biocomponents. Nano Lett. 2008, 8, 4469-4476.

97. Li, S.; Li, H.; Wang, X.; Song, Y.; Liu, Y.; Jiang, L.; Zhu, D. Super-Hydrophobicity of Large-Area Honeycomb-Like Aligned Carbon Nanotubes. J. Phys. Chem. B 2002, 106, 9274-9276.

98. Li, H.; Wang, X.; Song, Y.; Liu, Y.; Li, Q.; Jiang, L.; Zhu, D. Super-“Amphiphobic” Aligned Carbon Nanotube Films. Angew. Chem. Int. Ed. 2001, 40, 1743-1746.

99. Cao, P.; Xu, K.; Varghese, J. O.; Heath, J. R. The Microscopic Structure of Adsorbed Water on Hydrophobic Surfaces under Ambient Conditions. Nano Lett. 2011, 11, 55815586.

100. Luna, M.; Colchero, J.; Baró, A. M. Study of Water Droplets and Films on Graphite by Noncontact Scanning Force Microscopy. J. Phys. Chem. B 1999, 103, 9576-9581.

101. Martinez-Martin, D.; Longuinhos, R.; Izquierdo, J. G.; Marele, A.; Alexandre, S. S.; Jaafar, M.; Gómez-Rodríguez, J. M.; Bañares, L.; Soler, J. M.; Gomez-Herrero, J. Atmospheric Contaminants on Graphitic Surfaces. Carbon 2013, 61, 33-39.

102. Bourges-Monnier, C.; Shanahan, M. E. R. Influence of Evaporation on Contact Angle. Langmuir 1995, 11, 2820-2829.

103. Hu, H.; Larson, R. G. Evaporation of a Sessile Droplet on a Substrate. J. Phys. Chem. B 2002, 106, 1334-1344.

104. Shinozaki, A.; Arima, K.; Morita, M.; Kojima, I.; Azuma, Y. FTIR-ATR Evaluation of Organic Contaminant Cleaning Methods for $\mathrm{SiO}_{2}$ Surfaces. Anal. Sci. 2003, 19, 15571559.

105. Roche, A.; Wyon, C.; Marthon, S.; Ple, J. F.; Olivier, M.; Rochat, N.; Chabli, A.; Danel, A.; Juhel, M.; Tardif, F. Detection of Organic Contamination on Silicon Substrates: Comparison of Several Techniques. AIP Conference Proceedings 2001, 550, 297-301. 
106. Leitner, T.; Kattner, J.; Hoffmann, H. Infrared Reflection Spectroscopy of Thin Films on Highly Oriented Pyrolytic Graphite. Appl. Spectrosc. 2003, 57, 1502-1509.

107. Webb, M. J.; Palmgren, P.; Pal, P.; Karis, O.; Grennberg, H. A Simple Method to Produce Almost Perfect Graphene on Highly Oriented Pyrolytic Graphite. Carbon 2011, 49, 3242-3249.

108. Surwade, S. P.; Li, Z.; Liu, H. Thermal Oxidation and Unwrinkling of Chemical Vapor Deposition-Grown Graphene. J. Phys. Chem. C 2012, 116, 20600-20606.

109. Adamson, A. W. Physical Chemistry of Surfaces; John Wiley \& Sons, Inc.: New York, 1990; pp 403-408.

110. Edwards, R. S.; Coleman, K. S. Graphene Synthesis: Relationship to Applications. Nanoscale 2013, 5, 38-51.

111. Shih, C.-J.; Wang, Q. H.; Lin, S.; Park, K.-C.; Jin, Z.; Strano, M. S.; Blankschtein, D. Breakdown in the Wetting Transparency of Graphene. Phys. Rev. Lett. 2012, 109, 176101.

112. Wu, S. Polymer Interface and Adhesion. In Marcel Dekker: New York, 1982; pp 142146.

113. Strobel, M.; Corn, S.; Lyons, C. S.; Korba, G. A. Plasma Fluorination of Polyolefins. J. Polym. Sci., Part A: Polym. Chem. 1987, 25, 1295-1307.

114. Clouet, F.; Shi, M. K.; Prat, R.; Holl, Y.; Marie, P.; Leonard, D.; DePuydt, Y.; Bertrand, P.; Dewez, J.-L.; Doren, A. Plasma Surface Modification of Polymers: Relevance to Adhesion. In Strobel, M.; Lyons, C. S.; Mittal, K. L., Eds.; VSP: Utrecht, The Netherlands, 1994; pp 68.

115. Wu, S. Calculation of Interfacial Tension in Polymer Systems. Journal of Polymer Science C 1971, 34, 19-30.

116. Bunn, C. W.; Daubeny, R. P. The Unit Cell of Polyethylene. T. Faraday Soc. 1954, 50, 1173-1177.

117. Keller, A. Crystal Configurations and Their Relevance to the Crystalline Texture and Crystallization Mechanism in Polymers. Kolloid-Z.u.Z.Polymere 1964, 197, 98-115.

118. Poynor, A.; Hong, L.; Robinson, I. K.; Granick, S.; Zhang, Z.; Fenter, P. A. How Water Meets a Hydrophobic Surface. Phys. Rev. Lett. 2006, 97, 266101.

119. Yin, S.; Wang, C.; Qiu, X.; Xu, B.; Bai, C. Theoretical Study of the Effects of Intermolecular Interactions in Self-Assembled Long-Chain Alkanes Adsorbed on Graphite Surface. Surf. Interface Anal. 2001, 32, 248-252.

120. Geim, A.; Novoselov, K. S. The Rise of Graphene. Nature Mater. 2007, 6, 183-191. 
121. Zhu, Y.; Murali, S.; Cai, W.; Li, X.; Suk, J. W.; Potts, J. R.; Ruoff, R. S. Graphene and Graphene Oxide: Synthesis, Properties, and Applications. Adv. Mater. 2010, 22, 3906-24.

122. Zhu, Y.; Murali, S.; Stoller, M. D.; Ganesh, K. J.; Cai, W.; Ferreira, P. J.; Pirkle, A.; Wallace, R. M.; Cychosz, K. A.; Thommes, M.; Su, D.; Stach, E. A.; Ruoff, R. S. Carbon-Based Supercapacitors Produced by Activation of Graphene. Science 2011, 332, 1537-1541.

123. Rafiee, J.; Mi, X.; Gullapalli, H.; Thomas, A. V.; Yavari, F.; Shi, Y.; Ajayan, P. M.; Koratkar, N. A. Wetting Transparency of Graphene. Nature Mater. 2012, 11, 217-22.

124. Ghosh, S.; An, X.; Shah, R.; Rawat, D.; Dave, B.; Kar, S.; Talapatra, S. Effect of 1Pyrene Carboxylic-Acid Functionalization of Graphene on Its Capacitive Energy Storage. J. Phys. Chem. C 2012, 116, 20688-20693.

125. Selvakumar, N.; Barshilia, H. C.; Rajam, K. S. Effect of Substrate Roughness on the Apparent Surface Free Energy of Sputter Deposited Superhydrophobic Polytetrafluoroethylene Coatings: A Comparison of Experimental Data with Different Theoretical Models. J. Appl. Phys. 2010, 108, 013505.

126. Koenig, S. P.; Boddeti, N. G.; Dunn, M. L.; Bunch, J. S. Ultrastrong Adhesion of Graphene Membranes. Nat. Nanotechnol. 2011, 6, 543-6.

127. Schedin, F.; Geim, A.; Morozov, S. V.; Hill, E. W.; Blake, P.; Katsnelson, M. I.; Novoselov, K. S. Detection of Individual Gas Molecules Adsorbed on Graphene. Nature Mater. 2007, 6, 652-5.

128. Kim, K.-S.; Lee, H.-J.; Lee, C.; Lee, S.-K.; Jang, H.; Ahn, J.-H.; Kim, J.-H.; Lee, H.-J. Chemical Vapor Deposition-Grown Graphene: The Thinnest Solid Lubricant. ACS Nano 2011, 5, 5107-14.

129. Owens, D. K.; Wendt, R. C. Estimation of the Surface Free Energy of Polymers. J. Appl. Polym. Sci. 1969, 13, 1741-1747.

130. Li, D.; Neumann, A. W. Contact Angles on Hydrophobic Solid Surfaces and Their Interpretation. J. Colloid Interface Sci. 1992, 148, 190-200.

131. Zhu, H.; Qin, X.; Sun, X.; Yan, W.; Yang, J.; Xie, Y. Rocking-Chair Configuration in Ultrathin Lithium Vanadate-Graphene Hybrid Nanosheets for Electrical Modulation. Sci. Rep. 2013, 3, 1246.

132. Hernandez, Y.; Nicolosi, V.; Lotya, M.; Blighe, F. M.; Sun, Z.; De, S.; McGovern, I. T.; Holland, B.; Byrne, M.; Gun'Ko, Y. K.; Boland, J. J.; Niraj, P.; Duesberg, G.; Krishnamurthy, S.; Goodhue, R.; Hutchison, J.; Scardaci, V.; Ferrari, A. C.; Coleman, J. N. High-Yield Production of Graphene by Liquid-Phase Exfoliation of Graphite. Nat. Nanotechnol. 2008, 3, 563-568. 
133. Wang, Q. H.; Kalantar-Zadeh, K.; Kis, A.; Coleman, J. N.; Strano, M. S. Electronics and Optoelectronics of Two-Dimensional Transition Metal Dichalcogenides. Nat.

Nanotechnol. 2012, 7, 699-712.

134. Deshmukh, R. R.; Shetty, A. R. Comparison of Surface Energies Using Various Approaches and Their Suitability. J. Appl. Polym. Sci. 2008, 107, 3707-3717.

135. Kwok, D. Y.; Ng, H.; Neumann, A. W. Experimental Study on Contact Angle Patterns: Liquid Surface Tensions Less Than Solid Surface Tensions. J. Colloid Interface Sci. 2000, 225, 323-328.

136. Dalal, E. N. Calculation of Solid Surface Tensions. Langmuir 1987, 3, 1009-1015.

137. Żenkiewicz, M. Methods for the Calculation of Surface Free Energy of Solids. JAMME 2007, 24, 1-9.

138. Neumann, A. W.; Good, R. J.; Hope, C. J.; Sejpal, M. An Equation-of-State Approach to Determine Surface Tensions of Low-Energy Solids from Contact Angles. J. Colloid Interface Sci. 1974, 49, 291-304.

139. Fowkes, F. M. Attractive Forces at Interfaces. Ind. Eng. Chem. 1964, 56, 40-52.

140. Subedi, D. P. Contact Angle Measurement for the Surface Characterization of Solids. The Himalayan Physics 2011, 2, 1-4.

141. Young, T. An Essay on the Cohesion of Fluids. Philos. Trans. R. Soc. London 1805, 95, 65-87.

142. Kloubek, J. Development of Methods for Surface Free Energy Determination Using Contact Angles of Liquids on Solids. Adv. Colloid Interface Sci. 1992, 38, 99-142.

143. Sharma, P. K.; Rao, K. H. Analysis of Different Approaches for Evaluation of Surface Energy of Microbial Cells by Contact Angle Goniometry. Adv. Colloid Interface Sci. 2002, 98, 341-463.

144. Chibowski, E.; Perea-Carpio, R. Problems of Contact Angle and Solid Surface Free Energy Determination. Adv. Colloid Interface Sci. 2002, 98, 245-264.

145. Good, R. J. Contact Angle, Wetting, and Adhesion: A Critical Review. J. Adhes. Sci. Technol. 1992, 6, 1269-1302.

146. Kwok, D. Y.; Neumann, A. W. Contact Angle, Wettability and Adhesion; Mittall, K. L., Eds.; VSP International Science: Boston, 2003; pp 117.

147. Marcellin Berthelot. https://en.wikipedia.org/wiki/Marcellin_Berthelot (accessed March 22, 2016). 
148. Berthelot, D. Sur Le Mélange Des Gaz. Comptes Rendus Hebdomadaires des Séances de l'Académie des Sciences 1898, 126, 1703-1855.

149. Dupré, A.; Dupré, P. Théorie Mécanique De La Chaleur; Gauthier-Villan: Paris, 1869.

150. Helm, C. A. Agnes Pockels: Life, Letters, and Papers.

https://www.aps.org/programs/women/workshops/upload/helm.pdf (accessed February 11, 2016).

151. Derrick, M. E. Agnes Pockels, 1862-1935. J. Chem. Educ. 1982, 59, 1030.

152. Rayleigh. Surface Tension. Nature 1891, 43, 437-439.

153. Schrader, M. E. Young-Dupre Revisited. Langmuir 1995, 11, 3585-3589.

154. Adam, N. K.; Jessop, G. CCL.-Angles of Contact and Polarity of Solid Surfaces. J. Chem. Soc., Trans. 1925, 127, 1863-1868.

155. Pockels, A. Über Randwinkel Und Ausbreitung Von Flüssigkeiten Auf Festen Körpern. Physik. Z. 1914, 15, 39-46.

156. Girifalco, L. A.; Good, R. J. A Theory for the Estimation of Surface and Interfacial Energies. I. Derivation and Application to Interfacial Tension. J. Phys. Chem. 1957, 61, 904-909.

157. Li, D.; Neumann, A. W. Equation of State for Interfacial Tensions of Solid-Liquid Systems. Adv. Colloid Interface Sci. 1992, 39, 299-345.

158. Kwok, D. Y.; Neumann, A. W. Contact Angle Measurement and Contact Angle Interpretation. Adv. Colloid Interface Sci. 1999, 81, 167-249.

159. Żenkiewicz, M. Application of Neumann's Method for Examination of Surface Free Energy of Modified Polyethylene Foils. Archives of Materials Science 1989, 10, 43-54.

160. Żenkiewicz, M. New Method for Analyzing the Surface Free Energy of Polymeric Materials Calculated with Owens-Wendt and Neumann Methods. Polimery 2006, 51, 584-587.

161. Żenkiewicz, M. Comparative Study on the Surface Free Energy of a Solid Calculated by Different Methods. Polym. Test. 2007, 26, 14-19.

162. Fowkes, F. M. Additivity of Intermolecular Forces at Interfaces. I. Determination of the Contribution to Surface and Interfacial Tensions of Dispersion Forces in Various Liquids. J. Phys. Chem. 1963, 67, 2538-2541.

163. Fowkes, F. M. Donor-Acceptor Interactions at Interfaces. J. Adhesion 1972, 4, 155-159. 
164. Fowkes, F. M. Determination of Interfacial Tensions, Contact Angles, and Dispersion Forces in Surfaces by Assuming Additivity of Intermolecular Interactions in Surfaces. $J$. Phys. Chem. 1962, 66, 382-382.

165. Fowkes, F. M. Donor-Acceptor Interactions at Interfaces. In Adhesion and Adsorption of Polymers, Lee, L.-H., Ed. Springer US: Boston, MA, 1980; pp 43-52.

166. Siboni, S.; Della Volpe, C.; Maniglio, D.; Brugnara, M. The Solid Surface Free Energy Calculation: II. The Limits of the Zisman and of the "Equation-of-State" Approaches. $J$. Colloid Interface Sci. 2004, 271, 454-472.

167. Della Volpe, C.; Maniglio, D.; Brugnara, M.; Siboni, S.; Morra, M. The Solid Surface Free Energy Calculation: I. In Defense of the Multicomponent Approach. J. Colloid Interface Sci. 2004, 271, 434-453.

168. Ferrari, A. C.; Meyer, J. C.; Scardaci, V.; Casiraghi, C.; Lazzeri, M.; Mauri, F.; Piscanec, S.; Jiang, D.; Novoselov, K. S.; Roth, S.; Geim, A. K. Raman Spectrum of Graphene and Graphene Layers. Phys. Rev. Lett. 2006, 97.

169. Reina, A.; Jia, X.; Ho, J.; Nezich, D.; Son, H.; Bulovic, V.; Dresselhaus, M.; Kong, J. Large Area, Few-Layer Graphene Films on Arbitrary Substrates by Chemical Vapor Deposition. Nano Lett. 2009, 9, 30-35.

170. Hyatt, H. A.; Cherlow, J. M.; Fenner, W. R.; Porto, S. P. S. Cross Section for the Raman Effect in Molecular Nitrogen Gas. J. Opt. Soc. Am. 1973, 63, 1604-1606.

171. Johs, B.; Herzinger, C. M. Quantifying the Accuracy of Ellipsometer Systems. Physica Status Solidi C 2008, 5, 1031-1035.

172. Wenzel, R. Resistance of Solid Surfaces to Wetting by Water. Ind. Eng. Chem. 1936, 28, 988-994.

173. Ramón-Torregrosa, P. J.; Rodríguez-Valverde, M. A.; Amirfazli, A.; Cabrerizo-Vílchez, M. A. Factors Affecting the Measurement of Roughness Factor of Surfaces and Its Implications for Wetting Studies. Colloids Surf., A 2008, 323, 83-93.

174. Poon, C. Y.; Bhushan, B. Comparison of Surface Roughness Measurements by Stylus Profiler, AFM and Non-Contact Optical Profiler. Wear 1995, 190, 76-88.

175. Dhingra, S.; Hsu, J.-F.; Vlassiouk, I.; D'Urso, B. Chemical Vapor Deposition of Graphene on Large-Domain Ultra-Flat Copper. Carbon 2014, 69, 188-193.

176. Not So Transparent. Nature Mater. 2013, 12, 865.

177. Chibowski, E.; Terpilowski, K. Surface Free Energy of Sulfur-Revisited: I. Yellow and Orange Samples Solidified against Glass Surface. J. Colloid Interface Sci. 2008, 319, 505-513. 
178. Terpilowski, K.; Holysz, L.; Chibowski, E. Surface Free Energy of Sulfur-Revisited: II. Samples Solidified against Different Solid Surfaces. J. Colloid Interface Sci. 2008, 319, 514-519.

179. Sharma, M.; Donadio, D.; Schwegler, E.; Galli, G. Probing Properties of Water under Confinement: Infrared Spectra. Nano Lett. 2008, 8, 2959-2962.

180. Fukuzawa, K.; Shimuta, T.; Nakada, A.; Zhang, H.; Mitsuya, Y. Measurement of Thickness of Molecularly Thin Lubricant Film Using Ellipsometric Microscopy. IEEE Trans. Magn. 2005, 41, 808-811.

181. McMillan, T.; Rutledge, J. E.; Taborek, P. Ellipsometry of Liquid Helium Films on Gold, Cesium, and Graphite. J. Low Temp. Phys. 2005, 138, 995-1011.

182. Cassie, A. B. D.; Baxter, S. Wettability of Porous Surfaces. T. Faraday Soc. 1944, 40, 546-551.

183. Hamada, I. Adsorption of Water on Graphene: A van der Waals Density Functional Study. Phys. Rev. B 2012, 86.

184. Voloshina, E.; Usvyat, D.; Schutz, M.; Dedkov, Y.; Paulus, B. On the Physisorption of Water on Graphene: A CCSD(T) Study. Phys. Chem. Chem. Phys. 2011, 13, 12041-7.

185. Rubes, M.; Kysilka, J.; Nachtigall, P.; Bludsky, O. DFT/CC Investigation of Physical Adsorption on a Graphite (0001) Surface. Phys. Chem. Chem. Phys. 2010, 12, 6438-44.

186. Suzuki, S.; Green, P. G.; Bumgarner, R. E.; Dasgupta, S.; Goddard, W. A.; Blake, G. A. Benzene Forms Hydrogen Bonds with Water. Science 1992, 257, 942-945.

187. Ghaderi, N.; Peressi, M. First-Principle Study of Hydroxyl Functional Groups on Pristine, Defected Graphene, and Graphene Epoxide. The Journal of Physical Chemistry C 2010, $114,21625-21630$.

188. Xu, K.; Heath, J. R. Contact with What. Nature Mater. 2013, 12, 872-873.

189. Kidambi, P. R.; Bayer, B. C.; Blume, R.; Wang, Z. J.; Baehtz, C.; Weatherup, R. S.; Willinger, M. G.; Schloegl, R.; Hofmann, S. Observing Graphene Grow: CatalystGraphene Interactions During Scalable Graphene Growth on Polycrystalline Copper. Nano Lett. 2013, 13, 4769-78.

190. Kimouche, A.; Renault, O.; Samaddar, S.; Winkelmann, C.; Courtois, H.; Fruchart, O.; Coraux, J. Modulating Charge Density and Inelastic Optical Response in Graphene by Atmospheric Pressure Localized Intercalation through Wrinkles. Carbon 2014, 68, 73-79.

191. Tuinstra, F.; Koenig, J. L. Raman Spectrum of Graphite. J. Chem. Phys. 1970, 53, 11261130. 
192. Ferrari, A. C.; Robertson, J. Interpretation of Raman Spectra of Disordered and Amorphous Carbon. Phys. Rev. B 2000, 61, 14095-14107.

193. Das, A.; Chakraborty, B.; Sood, A. K. Raman Spectroscopy of Graphene on Different Substrates and Influence of Defects. Bull. Mater. Sci. 2008, 31, 579-584.

194. Cançado, L. G.; Jorio, A.; Ferreira, E. H. M.; Stavale, F.; Achete, C. A.; Capaz, R. B.; Moutinho, M. V. O.; Lombardo, A.; Kulmala, T. S.; Ferrari, A. C. Quantifying Defects in Graphene via Raman Spectroscopy at Different Excitation Energies. Nano Lett. 2011, 11, 3190-3196.

195. Pimenta, M. A.; Dresselhaus, G.; Dresselhaus, M. S.; Cançado, L. G.; Jorio, A.; Saito, R. Studying Disorder in Graphite-Based Systems by Raman Spectroscopy. Phys. Chem. Chem. Phys. 2007, 9, 1276-1290.

196. Jorio, A.; Saito, R.; Dresselhaus, G.; Dresselhaus, M. S. Dispersive G'-Band and HigherOrder Processes: The Double Resonance Process. In Raman Spectroscopy in Graphene Related Systems, Wiley-VCH Verlag GmbH \& Co. KGaA: 2011; pp 277-298.

197. Compagnini, G.; Puglisi, O.; Foti, G. Raman Spectra of Virgin and Damaged Graphite Edge Planes. Carbon 1997, 35, 1793-1797.

198. Zeng, J.; Yao, H. J.; Zhang, S. X.; Zhai, P. F.; Duan, J. L.; Sun, Y. M.; Li, G. P.; Liu, J. Swift Heavy Ions Induced Irradiation Effects in Monolayer Graphene and Highly Oriented Pyrolytic Graphite. Nucl. Instrum. Meth. B 2014, 330, 18-23.

199. Lucchese, M. M.; Stavale, F.; Ferreira, E. H. M.; Vilani, C.; Moutinho, M. V. O.; Capaz, R. B.; Achete, C. A.; Jorio, A. Quantifying Ion-Induced Defects and Raman Relaxation Length in Graphene. Carbon 2010, 48, 1592-1597.

200. Cançado, L. G.; Pimenta, M. A.; Neves, B. R. A.; Dantas, M. S. S.; Jorio, A. Influence of the Atomic Structure on the Raman Spectra of Graphite Edges. Phys. Rev. Lett. 2004, 93, 247401.

201. Casiraghi, C.; Hartschuh, A.; Qian, H.; Piscanec, S.; Georgi, C.; Fasoli, A.; Novoselov, K. S.; Basko, D. M.; Ferrari, A. C. Raman Spectroscopy of Graphene Edges. Nano Lett. 2009, 9, 1433-1441.

202. Krauss, B.; Nemes-Incze, P.; Skakalova, V.; Biro, L. P.; Klitzing, K. v.; Smet, J. H. Raman Scattering at Pure Graphene Zigzag Edges. Nano Lett. 2010, 10, 4544-4548.

203. Eckmann, A.; Felten, A.; Mishchenko, A.; Britnell, L.; Krupke, R.; Novoselov, K. S.; Casiraghi, C. Probing the Nature of Defects in Graphene by Raman Spectroscopy. Nano Lett. 2012, 12, 3925-3930.

204. Russell, A. M.; Lee, K. L. Defects and Their Effects on Materials Properties. In Structure-Property Relations in Nonferrous Metals; John Wiley \& Sons: Hoboken, NJ, 2005; pp 18-27. 
205. Pollard, A. J.; Brennan, B.; Stec, H.; Tyler, B. J.; Seah, M. P.; Gilmore, I. S.; Roy, D. Quantitative Characterization of Defect Size in Graphene Using Raman Spectroscopy. Appl. Phys. Lett. 2014, 105, 253107.

206. Knight, D. S.; White, W. B. Characterization of Diamond Films by Raman Spectroscopy. J. Mater. Res. 1989, 4, 385-393.

207. Lespade, P.; Marchand, A.; Couzi, M.; Cruege, F. Caracterisation De Materiaux Carbones Par Microspectrometrie Raman. Carbon 1984, 22, 375-385.

208. Ferrari, A. C. Raman Spectroscopy of Graphene and Graphite: Disorder, ElectronPhonon Coupling, Doping and Nonadiabatic Effects. Solid State Commun. 2007, 143, 4757.

209. Laor, U.; Schatz, G. C. The Role of Surface Roughness in Surface Enhanced Raman Spectroscopy (SERS): The Importance of Multiple Plasmon Resonances. Chem. Phys. Lett. 1981, 82, 566-570.

210. Kruszewskh, S.; Skonieczny, J. Roughness Effects in Surface Enhanced Raman Scattering - Evidence for Electromagnetic and Charge Transfer Enhancement Mechanism. Acta Phys. Pol., A 1991, 80, 611-620.

211. Hoffman, A. S.; Ratner, B. Lecture on Contact Angles. University of Washington, 2005. http://www.uweb.engr.washington.edu/education/pdf/ashsurfscontact\%20angles05.pdf (accessed December 17, 2014).

212. Isaac Childres; Jauregui, L. A.; Park, W.; Cao, H.; Chen, Y. P. Raman Spectroscopy of Graphene and Related Materials. In New Developments in Photon and Materials Research, Jang, J. I., Ed. Nova Science Publishers: 2013.

213. Lazzeri, M.; Piscanec, S.; Mauri, F.; Ferrari, A. C.; Robertson, J. Phonon Linewidths and Electron-Phonon Coupling in Graphite and Nanotubes. Phys. Rev. B 2006, 73, 155426.

214. Dresselhaus, M. S.; Kalish, R. Ion Implantation in Diamond, Graphite and Related Materials. In Springer Series in Materials Science; Springer-Verlag Berlin Heidelberg: 1992.

215. Mélinon, P.; Hannour, A.; Bardotti, L.; Prével, B.; Gierak, J.; Bourhis, E.; Faini, G.; Canut, B. Ion Beam Nanopatterning in Graphite: Characterization of Single Extended Defects. Nanotechnology 2008, 19, 235305.

216. Meng, X.; Tongay, S.; Kang, J.; Chen, Z.; Wu, F.; Li, S.-S.; Xia, J.-B.; Li, J.; Wu, J. Stable P- and N-Type Doping of Few-Layer Graphene/Graphite. Carbon 2013, 57, 507514.

217. Ferrari, A. C.; Basko, D. M. Raman Spectroscopy as a Versatile Tool for Studying the Properties of Graphene. Nat. Nanotechnol. 2013, 8, 235-246. 
218. Mignuzzi, S.; Kumar, N.; Brennan, B.; Gilmore, I. S.; Richards, D.; Pollard, A. J.; Roy, D. Probing Individual Point Defects in Graphene via near-Field Raman Scattering. Nanoscale 2015, 7, 19413-19418.

219. Mignuzzi, S.; Pollard, A. J.; Bonini, N.; Brennan, B.; Gilmore, I. S.; Pimenta, M. A.; Richards, D.; Roy, D. Effect of Disorder on Raman Scattering of Single-Layer $\mathrm{MoS}_{2}$. Phys. Rev. B 2015, 91, 195411.

220. Cho, C.; Gon Lee, Y.; Jung, U.; Goo Kang, C.; Lim, S.; Jun Hwang, H.; Choi, H.; Hun Lee, B. Correlation between the Hysteresis and the Initial Defect Density of Graphene. Appl. Phys. Lett. 2013, 103, 083110.

221. Beams, R.; Cançado, L. G.; Novotny, L. Raman Characterization of Defects and Dopants in Graphene. J. Phys.: Condens. Matter 2015, 27, 083002.

222. Chang, H.; Bard, A. J. Observation and Characterization by Scanning Tunneling Microscopy of Structures Generated by Cleaving Highly Oriented Pyrolytic Graphite. Langmuir 1991, 7, 1143-1153.

223. Mizes, H. A.; Harrison, W. A. The Signature of Point Defects in Layered Materials. $J$. Vac. Sci. Technol., A 1988, 6, 300-304.

224. Soto, M. R. The Effect of Point Defects on the STM Image of Graphite. Surf. Sci. 1990, 225, 190-194.

225. McCreery, R. L. Raman Microscopy and Imaging. In Raman Spectroscopy for Chemical Analysis, John Wiley \& Sons, Inc.: 2005; pp 293-332.

226. Mallet-Ladeira, P.; Puech, P.; Toulouse, C.; Cazayous, M.; Ratel-Ramond, N.; Weisbecker, P.; Vignoles, G. L.; Monthioux, M. A Raman Study to Obtain Crystallite Size of Carbon Materials: A Better Alternative to the Tuinstra-Koenig Law. Carbon 2014, 80, 629-639.

227. Spori, D. M. Structural Influences on Self-Cleaning Surfaces. ETH Zurich, 2010. http://dx.doi.org/10.3929/ethz-a-006193586

228. McHale, G. Cassie and Wenzel: Were They Really So Wrong? Langmuir 2007, 23, 8200-8205.

229. Wilson, N. R.; Guille, M.; Dumitrescu, I.; Fernandez, V. R.; Rudd, N. C.; Williams, C. G.; Unwin, P. R.; Macpherson, J. V. Assessment of the Electrochemical Behavior of Two-Dimensional Networks of Single-Walled Carbon Nanotubes. Anal. Chem. 2006, 78, 7006-7015.

230. Ashraf, A.; Wu, Y.; Wang, M. C.; Aluru, N. R.; Dastgheib, S. A.; Nam, S. Spectroscopic Investigation of the Wettability of Multilayer Graphene Using Highly Ordered Pyrolytic Graphite as a Model Material. Langmuir 2014, 30, 12827-36. 
231. Kozubek, R.; Ochedowski, O.; Zagoranskiy, I.; Karlušić, M.; Schleberger, M. Application of HOPG and CVD Graphene as Ion Beam Detectors. Nucl. Instrum. Meth. B 2014, 340, 81-84.

232. Heine, D. R.; Grest, G. S.; Webb, E. B. Surface Wetting of Liquid Nanodroplets: Droplet-Size Effects. Phys. Rev. Lett. 2005, 95, 107801.

233. Wang, R.; Bai, S. Effect of Droplet Size on Wetting Behavior on Laser Textured SiC Surface. Appl. Surf. Sci. 2015, 353, 564-567.

234. Yen, T.-H. Wetting Characteristics of Nanoscale Water Droplet on Silicon Substrates with Effects of Surface Morphology. Molec. Simul. 2011, 37, 766-778.

235. Katz, D. L.; Saltman, W. Surface Tension of Hydrocarbons. Ind. Eng. Chem. 1939, 31, 91-94.

236. Mangolini, F.; McClimon, J. B.; Rose, F.; Carpick, R. W. Accounting for NanometerThick Adventitious Carbon Contamination in X-Ray Absorption Spectra of CarbonBased Materials. Anal. Chem. 2014, 86, 12258-12265.

237. Shih, C.-J.; Strano, M. S.; Blankschtein, D. Wetting Translucency of Graphene. Nature Mater. 2013, 12, 866-869.

238. Parobek, D.; Liu, H. Wettability of Graphene. 2D Mater. 2015, 2, 032001.

239. Amadei, C. A.; Lai, C.-Y.; Esplandiu, M. J.; Alzina, F.; Vecitis, C. D.; Verdaguer, A.; Chiesa, M. Elucidation of the Wettability of Graphene through a Multi-Length-Scale Investigation Approach. RSC Adv. 2015, 5, 39532-39538.

240. Ramos-Alvarado, B.; Kumar, S.; Peterson, G. P. On the Wettability Transparency of Graphene-Coated Silicon Surfaces. J. Chem. Phys. 2016, 144, 014701.

241. Wei, Y.; Jia, C. Q. Intrinsic Wettability of Graphitic Carbon. Carbon 2015, 87, 10-17.

242. Marbou, K.; Al Ghaferi, A.; Jouiad, M. In-Situ Characterization of Wettability Alteration in HOPG. SOP T. Nanotechnol., in press.

243. Aria, A. I.; Kidambi, P. R.; Weatherup, R. S.; Xiao, L.; Williams, J. A.; Hofmann, S. Time Evolution of the Wettability of Supported Graphene under Ambient Air Exposure. J. Phys. Chem. C 2016, 120, 2215-2224.

244. Gao, L.; McCarthy, T. J. “Artificial Lotus Leaf” Prepared Using a 1945 Patent and a Commercial Textile. Langmuir 2006, 22, 5998-6000.

245. Chibowski, E.; Ontiveros-Ortega, A.; Perea-Carpio, R. On the Interpretation of Contact Angle Hysteresis. J. Adhes. Sci. Technol. 2002, 16, 1367-1404. 
246. Chibowski, E.; Jurak, M. Comparison of Contact Angle Hysteresis of Different Probe Liquids on the Same Solid Surface. Colloid. Polym. Sci. 2012, 291, 391-399.

247. Diaz, M. E.; Fuentes, J.; Cerro, R. L.; Savage, M. D. Hysteresis During Contact Angles Measurement. J. Colloid Interface Sci. 2010, 343, 574-583.

248. Eral, H. B.; 't Mannetje, D. J. C. M.; Oh, J. M. Contact Angle Hysteresis: A Review of Fundamentals and Applications. Colloid. Polym. Sci. 2012, 291, 247-260.

249. Decker, E. L.; Frank, B.; Suo, Y.; Garoff, S. Physics of Contact Angle Measurement. Colloids Surf., A 1999, 156, 177-189.

250. Erbil, H. Y. The Debate on the Dependence of Apparent Contact Angles on Drop Contact Area or Three-Phase Contact Line: A Review. Surf. Sci. Rep. 2014, 69, 325-365.

251. Raj, R.; Enright, R.; Zhu, Y.; Adera, S.; Wang, E. N. Unified Model for Contact Angle Hysteresis on Heterogeneous and Superhydrophobic Surfaces. Langmuir 2012, 28, 15777-15788.

252. Tadmor, R. Line Energy and the Relation between Advancing, Receding, and Young Contact Angles. Langmuir 2004, 20, 7659-7664.

253. Larsen, S. T.; Taboryski, R. A Cassie-Like Law Using Triple Phase Boundary Line Fractions for Faceted Droplets on Chemically Heterogeneous Surfaces. Langmuir 2009, 25, 1282-1284.

254. Rivetti, M.; Teisseire, J.; Barthel, E. Surface Fraction Dependence of Contact Angles Induced by Kinks in the Triple Line. Phys. Rev. Lett. 2015, 115, 016101.

255. Kashaninejad, N.; Chan, W. K.; Nguyen, N.-T. Eccentricity Effect of Micropatterned Surface on Contact Angle. Langmuir 2012, 28, 4793-4799.

256. Murakami, D.; Jinnai, H.; Takahara, A. Wetting Transition from the Cassie-Baxter State to the Wenzel State on Textured Polymer Surfaces. Langmuir 2014, 30, 2061-2067.

257. Housecroft, C. E.; Sharpe, A. G. Inorganic Chemistry; Ashford Colour Press Ltd: Gosport, United Kingdom, 2005.

258. Oxtoby, D. W.; Gillis, H. P.; Campion, A. Principles of Modern Chemistry; Thomson Brooks/Cole: United States, 2008.

259. Raj, R.; Adera, S.; Enright, R.; Wang, E. N. High-Resolution Liquid Patterns via ThreeDimensional Droplet Shape Control. Nat. Commun. 2014, 5.

260. Extrand, C. W. Contact Angles and Hysteresis on Surfaces with Chemically Heterogeneous Islands. Langmuir 2003, 19, 3793-3796. 
261. Gao, L.; McCarthy, T. J. How Wenzel and Cassie Were Wrong. Langmuir 2007, 23, $3762-3765$.

262. Gao, L.; McCarthy, T. J. Wetting 101 . Langmuir 2009, 25, 14105-14115.

263. Chibowski, E. Surface Free Energy of a Solid from Contact Angle Hysteresis. Adv. Colloid Interface Sci. 2003, 103, 149-172.

264. R.N. Wenzel, Former Mellon Chemist, Dies. Pittsburgh Press February 5, 1979, https://news.google.com/newspapers?id=o3cqAAAAIBAJ\&sjid=BVkEAAAAIBAJ\&pg $=7057 \% 2 \mathrm{C} 1634631$.

265. Pease, D. C. The Significance of the Contact Angle in Relation to the Solid Surface. $J$. Phys. Chem. 1945, 49, 107-110.

266. Bartell, F. E.; Shepard, J. W. Surface Roughness as Related to Hysteresis of Contact Angles. II. The Systems Paraffin-3 Molar Calcium Chloride Solution-Air and ParaffinGlycerol-Air. J. Phys. Chem. 1953, 57, 455-458.

267. Extrand, C. W.; Moon, S. I. Which Controls Wetting? Contact Line Versus Interfacial Area: Simple Experiments on Capillary Rise. Langmuir 2012, 28, 15629-15633.

268. Gao, L.; McCarthy, T. J. An Attempt to Correct the Faulty Intuition Perpetuated by the Wenzel and Cassie "Laws". Langmuir 2009, 25, 7249-7255.

269. Panchagnula, M. V.; Vedantam, S. Comment on How Wenzel and Cassie Were Wrong by Gao and Mccarthy. Langmuir 2007, 23, 13242-13242.

270. Israelachvili, J. N.; Gee, M. L. Contact Angles on Chemically Heterogeneous Surfaces. Langmuir 1989, 5, 288-289.

271. Choi, W.; Tuteja, A.; Mabry, J. M.; Cohen, R. E.; McKinley, G. H. A Modified CassieBaxter Relationship to Explain Contact Angle Hysteresis and Anisotropy on NonWetting Textured Surfaces. J. Colloid Interface Sci. 2009, 339, 208-216.

272. Li, W.; Amirfazli, A. A Thermodynamic Approach for Determining the Contact Angle Hysteresis for Superhydrophobic Surfaces. J. Colloid Interface Sci. 2005, 292, 195-201.

273. Reyssat, M.; Quéré, D. Contact Angle Hysteresis Generated by Strong Dilute Defects. $J$. Phys. Chem. B 2009, 113, 3906-3909.

274. Yeh, K.-Y.; Chen, L.-J.; Chang, J.-Y. Contact Angle Hysteresis on Regular Pillar-Like Hydrophobic Surfaces. Langmuir 2008, 24, 245-251.

275. Yu, Y.; Wu, Q.; Zhang, K.; Ji, B. Effect of Triple-Phase Contact Line on Contact Angle Hysteresis. Sci. China: Phys., Mech. Astron. 2012, 55, 1045-1050. 
276. Zheng, Q. S.; Yu, Y.; Zhao, Z. H. Effects of Hydraulic Pressure on the Stability and Transition of Wetting Modes of Superhydrophobic Surfaces. Langmuir 2005, 21, 1220712212.

277. McHale, G.; Newton, M. I.; Shirtcliffe, N. J. Dynamic Wetting and Spreading and the Role of Topography. J. Phys.: Condens. Matter 2009, 21, 464122.

278. Li, R.; Shan, Y. Contact Angle and Local Wetting at Contact Line. Langmuir 2012, 28, 15624-15628.

279. Marmur, A.; Bittoun, E. When Wenzel and Cassie Are Right: Reconciling Local and Global Considerations. Langmuir 2009, 25, 1277-1281.

280. Fadeev, A. Y.; McCarthy, T. J. Trialkylsilane Monolayers Covalently Attached to Silicon Surfaces: Wettability Studies Indicating That Molecular Topography Contributes to Contact Angle Hysteresis. Langmuir 1999, 15, 3759-3766.

281. Adamson, A. W.; Gast, A. P. Wetting, Flotation, and Detergency. In Physical Chemistry of Surfaces; John Wiley \& Sons: New York, 1997; pp 465-499.

282. de Gennes, P. G. Wetting: Statics and Dynamics. Rev. Mod. Phys. 1985, 57, 827-863.

283. de Gennes, P. G.; Brochard-Wyart, F.; Quere, D. Capillarity and Wetting Phenomena; Springer: New York, 2003.

284. Israelachvili, J. Intermolecular \& Surface Forces; Academic Press Limited: London, 1991.

285. Dickinson, R. G.; Pauling, L. The Crystal Structure of Molybdenite. J. Am. Chem. Soc. 1923, 45, 1466-1471.

286. Novoselov, K. S.; Jiang, D.; Schedin, F.; Booth, T. J.; Khotkevich, V. V.; Morozov, S. V.; Geim, A. K. Two-Dimensional Atomic Crystals. Proc. Natl. Acad. Sci. U.S.A. 2005, $102,10451-10453$.

287. Graphene Is Not Alone. Nat. Nanotechnol. 2012, 7, 683.

288. Akinwande, D.; Petrone, N.; Hone, J. Two-Dimensional Flexible Nanoelectronics. Nat. Commun. 2014, 5, 5678 .

289. Lee, J.; Dak, P.; Lee, Y.; Park, H.; Choi, W.; Alam, M. A.; Kim, S. Two-Dimensional Layered $\mathrm{MoS}_{2}$ Biosensors Enable Highly Sensitive Detection of Biomolecules. Sci. Rep. 2014, 4, 7352 .

290. Splendiani, A.; Sun, L.; Zhang, Y.; Li, T.; Kim, J.; Chim, C. Y.; Galli, G.; Wang, F. Emerging Photoluminescence in Monolayer $\mathrm{MoS}_{2}$. Nano Lett. 2010, 10, 1271-5. 
291. Yazyev, O. V.; Kis, A. $\mathrm{MoS}_{2}$ and Semiconductors in the Flatland. Mater. Today 2015, 18, 20-30.

292. Sarkar, D.; Liu, W.; Xie, X.; Anselmo, A. C.; Mitragotri, S.; Banerjee, K. MoS ${ }_{2}$ FieldEffect Transistor for Next-Generation Label-Free Biosensors. ACS Nano 2014, 8, 39924003.

293. Jiang, S.; Cheng, R.; Ng, R.; Huang, Y.; Duan, X. Highly Sensitive Detection of Mercury(II) Ions with Few-Layer Molybdenum Disulfide. Nano Res. 2015, 8, 257-262.

294. Kelebek, S. Critical Surface Tension of Wetting and of Floatability of Molybdenite and Sulfur. J. Colloid Interface Sci. 1988, 124, 504-514.

295. Zhang, S.; Zeng, X.; Tang, Z.; Tan, M. J. Exploring the Antisticking Properties of Solid Lubricant Thin Films in Transfer Molding. Int. J. Mod Phys B 2002, 16, 1080-1085.

296. Gaur, A. P.; Sahoo, S.; Ahmadi, M.; Dash, S. P.; Guinel, M. J.; Katiyar, R. S. Surface Energy Engineering for Tunable Wettability through Controlled Synthesis of $\mathrm{MoS}_{2}$. Nano Lett. 2014, 14, 4314-21.

297. Chow, P. K.; Singh, E.; Viana, B. C.; Gao, J.; Luo, J.; Li, J.; Lin, Z.; Elías, A. L.; Shi, Y.; Wang, Z.; Terrones, M.; Koratkar, N. Wetting of Mono and Few-Layered $\mathrm{WS}_{2}$ and $\mathrm{MoS}_{2}$ Films Supported on $\mathrm{Si} / \mathrm{SiO}_{2}$ Substrates. ACS Nano 2015, 9, 3023-3031.

298. Ambrosi, A.; Sofer, Z.; Pumera, M. Lithium Intercalation Compound Dramatically Influences the Electrochemical Properties of Exfoliated $\mathrm{MoS}_{2}$. Small 2015, 11, 605-612.

299. Keong Koh, E. W.; Chiu, C. H.; Lim, Y. K.; Zhang, Y.-W.; Pan, H. Hydrogen Adsorption on and Diffusion through $\mathrm{MoS}_{2}$ Monolayer: First-Principles Study. Int. $J$. Hydrogen Energy 2012, 37, 14323-14328.

300. Dresselhaus, M. S. Intercalation in Layered Materials; Plenum Press: New York, 1986.

301. Wilson, J. A.; Yoffe, A. D. The Transition Metal Dichalcogenides Discussion and Interpretation of the Observed Optical, Electrical and Structural Properties. Adv. Phys. 1969, 18, 193-335.

302. Wang, H.; Yuan, H.; Sae Hong, S.; Li, Y.; Cui, Y. Physical and Chemical Tuning of Two-Dimensional Transition Metal Dichalcogenides. Chem. Soc. Rev. 2015, 44, 26642680.

303. Barrado, A. I.; García, S.; Barrado, E.; Pérez, R. M. PM 2.5 -bound PAHs and HydroxyPAHs in Atmospheric Aerosol Samples: Correlations with Season and with Physical and Chemical Factors. Atmos. Environ. 2012, 49, 224-232.

304. Srogi, K. Monitoring of Environmental Exposure to Polycyclic Aromatic Hydrocarbons: A Review. Environ. Chem. Lett. 2007, 5, 169-195. 
305. Prevedouros, K.; Brorström-Lundén, E.; J. Halsall, C.; Jones, K. C.; Lee, R. G. M.; Sweetman, A. J. Seasonal and Long-Term Trends in Atmospheric PAH Concentrations: Evidence and Implications. Environ. Pollut. 2004, 128, 17-27.

306. Tang, N.; Hattori, T.; Taga, R.; Igarashi, K.; Yang, X.; Tamura, K.; Kakimoto, H.; Mishukov, V. F.; Toriba, A.; Kizu, R.; Hayakawa, K. Polycyclic Aromatic Hydrocarbons and Nitropolycyclic Aromatic Hydrocarbons in Urban Air Particulates and Their Relationship to Emission Sources in the Pan-Japan Sea Countries. Atmos. Environ. 2005, $39,5817-5826$.

307. Li, Z.; Sjodin, A.; Porter, E. N.; Patterson Jr, D. G.; Needham, L. L.; Lee, S.; Russell, A. G.; Mulholland, J. A. Characterization of $\mathrm{PM}_{2.5}$-bound Polycyclic Aromatic Hydrocarbons in Atlanta. Atmos. Environ. 2009, 43, 1043-1050.

308. Wang, G.; Kawamura, K.; Zhao, X.; Li, Q.; Dai, Z.; Niu, H. Identification, Abundance and Seasonal Variation of Anthropogenic Organic Aerosols from a Mega-City in China. Atmos. Environ. 2007, 41, 407-416.

309. De Nicola, F.; Maisto, G.; Prati, M. V.; Alfani, A. Temporal Variations in PAH Concentrations in Quercus ilex L. (Holm Oak) Leaves in an Urban Area. Chemosphere 2005, 61, 432-440.

310. Nakajima, D.; Yoshida, Y.; Suzuki, J.; Suzuki, S. Seasonal Changes in the Concentration of Polycyclic Aromatic Hydrocarbons in Azalea Leaves and Relationship to Atmospheric Concentration. Chemosphere 1995, 30, 409-418.

311. Lodovici, M.; Dolara, P.; Taiti, S.; Del Carmine, P.; Bernardi, L.; Agati, L.; Ciappellano, S. Polynuclear Aromatic Hydrocarbons in the Leaves of the Evergreen Tree Laurus nobilis. Sci. Total Environ. 1994, 153, 61-68.

312. Nguyen, A.; Sharma, P.; Scott, T.; Preciado, E.; Klee, V.; Sun, D.; Lu, I. H.; Barroso, D.; Kim, S.; Shur, V. Y.; Akhmatkhanov, A. R.; Gruverman, A.; Bartels, L.; Dowben, P. A. Toward Ferroelectric Control of Monolayer $\mathrm{MoS}_{2}$. Nano Lett. 2015.

313. Santos, E. J. G.; Kaxiras, E. Electrically Driven Tuning of the Dielectric Constant in $\mathrm{MoS}_{2}$ Layers. ACS Nano 2013, 7, 10741-10746.

314. Graupe, M.; Takenaga, M.; Koini, T.; Colorado, R.; Lee, T. R. Oriented Surface Dipoles Strongly Influence Interfacial Wettabilities. J. Am. Chem. Soc. 1999, 121, 3222-3223.

315. Xu, J.; Cao, X. Characterization and Mechanism of $\mathrm{MoS}_{2} / \mathrm{CdS}$ Composite Photocatalyst Used for Hydrogen Production from Water Splitting under Visible Light. Chem. Eng. J. 2015, 260, 642-648.

316. Polyakov, M.; Vandenberg, M.; Hanft, T.; Poisot, M.; Bensch, W.; Muhler, M.; Grunert, W. Hydrocarbon Reactions on $\mathrm{MoS}_{2}$ Revisited, I: Activation of $\mathrm{MoS}_{2}$ and Interaction with Hydrogen Studied by Transient Kinetic Experiments. J. Catal. 2008, 256, 126-136. 
317. Tan, S. M.; Ambrosi, A.; Sofer, Z.; Huber, Š.; Sedmidubský, D.; Pumera, M. Pristine Basal- and Edge-Plane-Oriented Molybdenite $\mathrm{MoS}_{2}$ Exhibiting Highly Anisotropic Properties. Chem. Eur. J. 2015, 21, 7170-7178.

318. Pyrolytic Graphite Plate.

https://www.graphitestore.com/itemDetails.asp?item_id=3454\&prd_id=483\&cat_id=46\& curPage $=1$ (accessed September 2, 2014).

319. Koster, G.; Rijnders, G. In Situ Characterization of Thin Film Growth; Woodhead Publishing: Cambridge, 2011.

320. Nham, H. S.; Hess, G. B. Ellipsometric Study of Krypton, Methane, and Argon Films on Graphite: How Complete Is Wetting? Langmuir 1989, 5, 575-582.

321. Prunici, P.; Hess, P. Ellipsometric in Situ Measurement of Oxidation Kinetics and Thickness of (C2 - C20) Alkylsilyl (Sub)Monolayers. J. Appl. Phys. 2008, 103, 0243127.

322. Zhao, S.; Surwade, S. P.; Li, Z.; Liu, H. Photochemical Oxidation of CVD-Grown Single Layer Graphene. Nanotechnology 2012, 23, 355703.

323. Johs, B.; Hale, J. S. Dielectric Function Representation by B-Splines. Physica Status Solidi A 2008, 205, 715-719.

324. Wall, J. F.; Brumfield, J. C.; Murray, R. W.; Irene, E. A. Spectroscopic Ellipsometry and Atomic Force Microscopy of Polyphenylene Oxide Films. J. Electrochem. Soc. 1994, $141,306-310$.

325. Kattner, J.; Hoffmann, H. Simultaneous Determination of Thicknesses and Refractive Indices of Ultrathin Films by Multiple Incidence Medium Ellipsometry. J. Phys. Chem. B 2002, 106, 9723-9729.

326. Lama, R. F.; Lu, B. C. Y. Refractive Indices of Binary Hydrocarbon Mixtures. J. Chem. Eng. Data 1966, 11, 47-49.

327. O'Brien, R. N.; Quon, D. Refractive Index of Some Alcohols and Saturated Hydrocarbons at 6328 A. J. Chem. Eng. Data 1968, 13, 517-517.

328. Freund, A. K.; Munkholm, A.; Brennan, S. X-Ray Diffraction Properties of Highly Oriented Pyrolytic Graphite. 1996, 2856, 68-79.

329. Kim, E.; Oh, I.; Kwak, J. Atomic Structure of Highly Ordered Pyrolytic Graphite Doped with Boron. Electrochem. Commun. 2001, 3, 608-612.

330. Taylor, D. Library of Excel Routines.

http://ig.crystallography.org.uk/news/n00t1.htm\#spread (accessed February 12, 2016). 
331. Scientific Background on the Nobel Prize in Physics 2010: Graphene. https://www.nobelprize.org/nobel_prizes/physics/laureates/2010/advancedphysicsprize2010.pdf (accessed February 15, 2016).

332. The Nobel Prize in Physics 1915.

http://www.nobelprize.org/nobel_prizes/physics/laureates/1915/ (accessed February 9, 2016).

333. Bragg, W. H.; Bragg, W. L. The Reflection of X-Rays by Crystals. Proc. R. Soc. A 1913, $88,428-438$.

334. Meyers, H. P. Introductory Solid State Physics; CRC Press: 1997.

335. Speranza, G.; Minati, L. The Surface and Bulk Core Lines in Crystalline and Disordered Polycrystalline Graphite. Surf. Sci. 2006, 600, 4438-4444.

336. Dimitriou, M. D.; Kramer, E. J.; Hawker, C. J. Advanced Techniques for the Characterization of Surface Structure in Polymer Thin Films and Coatings. Arab. J. Sci. Eng. 2013, 39, 1-13.

337. Lascovich, J. C.; Scaglione, S. Comparison among XAES, PELS and XPS Techniques for Evaluation of sp ${ }^{2}$ Percentage in a-C:H. Appl. Surf. Sci. 1994, 78, 17-23.

338. McFeely, F. R.; Kowalczyk, S. P.; Ley, L.; Cavell, R. G.; Pollak, R. A.; Shirley, D. A. XRay Photoemission Studies of Diamond, Graphite, and Glassy Carbon Valence Bands. Phys. Rev. B 1974, 9, 5268-5278.

339. Schäfer, J.; Ristein, J.; Graupner, R.; Ley, L.; Stephan, U.; Frauenheim, T.; Veerasamy, V. S.; Amaratunga, G. A. J.; Weiler, M.; Ehrhardt, H. Photoemission Study of Amorphous Carbon Modifications and Comparison with Calculated Densities of States. Phys. Rev. B 1996, 53, 7762-7774.

340. Endo, K.; Koizumi, S.; Otsuka, T.; Suhara, M.; Morohasi, T.; Kurmaev, E. Z.; Chong, D. P. Analysis of XPS and XES of Diamond and Graphite by DFT Calculations Using Model Molecules. J. Comput. Chem. 2001, 22, 102-108.

341. Marchon, B.; Carrazza, J.; Heinemann, H.; Somorjai, G. A. TPD and XPS Studies of $\mathrm{O}_{2}$, $\mathrm{CO}_{2}$, and $\mathrm{H}_{2} \mathrm{O}$ Adsorption on Clean Polycrystalline Graphite. Carbon 1988, 26, 507-514.

342. Barber, M.; Evans, E. L.; Thomas, J. M. Oxygen Chemisorption on the Basal Faces of Graphite: An XPS Study. Chem. Phys. Lett. 1973, 18, 423-425.

343. Yang, Y.; Murali, R. Binding Mechanisms of Molecular Oxygen and Moisture to Graphene. Appl. Phys. Lett. 2011, 98, 093116.

344. Ryu, S.; Liu, L.; Berciaud, S.; Yu, Y.-J.; Liu, H.; Kim, P.; Flynn, G. W.; Brus, L. E. Atmospheric Oxygen Binding and Hole Doping in Deformed Graphene on a $\mathrm{SiO}_{2}$ Substrate. Nano Lett. 2010, 10, 4944-4951. 
345. Lazar, P.; Karlický, F.; Jurečka, P.; Kocman, M.; Otyepková, E.; Šafářová, K.; Otyepka, M. Adsorption of Small Organic Molecules on Graphene. J. Am. Chem. Soc. 2013, 135, 6372-6377.

346. Speranza, G.; Laidani, N. Measurement of the Relative Abundance of $\mathrm{sp}^{2}$ and $\mathrm{sp}^{3}$ Hybridised Atoms in Carbon-Based Materials by XPS: A Critical Approach. Part II. Diamond Relat. Mater. 2004, 13, 451-458.

347. Mizokawa, Y.; Miyasato, T.; Nakamura, S.; Geib, K. M.; Wilmsen, C. W. Comparison of the CKLL First-Derivative Auger Spectra from XPS and AES Using Diamond, Graphite, $\mathrm{SiC}$ and Diamond-Like-Carbon Films. Surf. Sci. 1987, 182, 431-438.

348. Kaciulis, S.; Mezzi, A.; Calvani, P.; Trucchi, D. M. Electron Spectroscopy of the Main Allotropes of Carbon. Surf. Interface Anal. 2014, 46, 966-969.

349. Kaciulis, S. Spectroscopy of Carbon: From Diamond to Nitride Films. Surf. Interface Anal. 2012, 44, 1155-1161.

350. XPS Simplified: Carbon. http://xpssimplified.com/elements/carbon.php (accessed September 3, 2015).

351. Lesiak, B.; Zemek, J.; Houdkova, J.; Kromka, A.; Jóźwik, A. Electron Spectra Line Shape Analysis of Highly Oriented Pyrolytic Graphite and Nanocrystalline Diamond. Anal. Sci. 2010, 26, 217-222.

352. Haerle, R.; Riedo, E.; Pasquarello, A.; Baldereschi, A. $s p^{2} / s p^{3}$ Hybridization Ratio in Amorphous Carbon from C 1s Core-Level Shifts: X-Ray Photoelectron Spectroscopy and First-Principles Calculation. Phys. Rev. B 2001, 65, 045101.

353. Zemek, J.; Zalman, J.; Luches, A. XAES and XPS Study of Amorphous Carbon Nitride Layers. Appl. Surf. Sci. 1998, 133, 27-32.

354. Titantah, J. T.; Lamoen, D. $\mathrm{sp}^{3} / \mathrm{sp}^{2}$ Characterization of Carbon Materials from FirstPrinciples Calculations: X-Ray Photoelectron Versus High Energy Electron Energy-Loss Spectroscopy Techniques. Carbon 2005, 43, 1311-1316.

355. Popov, C.; Kulisch, W.; Bliznakov, S.; Mednikarov, B.; Spasov, G.; Pirov, J.; Jelinek, M.; Kocourek, T.; Zemek, J. Characterization of the Bonding Structure of Nanocrystalline Diamond and Amorphous Carbon Films Prepared by Plasma Assisted Techniques. Appl. Phys. A 2007, 89, 209-212.

356. Speranza, G.; Minati, L.; Anderle, M. The C1s Core Line in Irradiated Graphite. J. Appl. Phys. 2007, 102, 043504.

357. Susi, T.; Pichler, T.; Ayala, P. X-Ray Photoelectron Spectroscopy of Graphitic Carbon Nanomaterials Doped with Heteroatoms. Beilstein J. Nanotechnol. 2015, 6, 177-192. 
358. Smith, R. A. P.; Armstrong, C. W.; Smith, G. C.; Weightman, P. Observation of a Surface Chemical Shift in Carbon $1 s$ Core-Level Photoemission from Highly Oriented Pyrolytic Graphite. Phys. Rev. B 2002, 66, 245409.

359. Doniach, S.; Sunjic, M. Many-Electron Singularity in X-Ray Photoemission and X-Ray Line Spectra from Metals. J. Phys. C 1970, 3, 285.

360. Yang, D.-Q.; Sacher, E. Carbon 1s X-Ray Photoemission Line Shape Analysis of Highly Oriented Pyrolytic Graphite: The Influence of Structural Damage on Peak Asymmetry. Langmuir 2006, 22, 860-862.

361. Hunt, M. R. C. Surface and Bulk Components in Angle-Resolved Core-Level Photoemission Spectroscopy of Graphite. Phys. Rev. B 2008, 78, 153408.

362. Yang, D.-Q.; Rochette, J.-F.; Sacher, E. Controlled Chemical Functionalization of Multiwalled Carbon Nanotubes by Kiloelectronvolt Argon Ion Treatment and Air Exposure. Langmuir 2005, 21, 8539-8545.

363. Yang, D. Q.; Sacher, E. s-p Hybridization in Highly Oriented Pyrolytic Graphite and Its Change on Surface Modification, as Studied by X-Ray Photoelectron and Raman Spectroscopies. Surf. Sci. 2002, 504, 125-137.

364. Yang, D. Q.; Sacher, E. $\mathrm{Ar}^{+}-$Induced Surface Defects on HOPG and Their Effect on the Nucleation, Coalescence and Growth of Evaporated Copper. Surf. Sci. 2002, 516, 43-55.

365. Cheung, T. T. P. X-Ray Photoemission of Carbon: Lineshape Analysis and Application to Studies of Coals. J. Appl. Phys. 1982, 53, 6857-6862.

366. Nozières, P.; De Dominicis, C. T. Singularities in the X-Ray Absorption and Emission of Metals. III. One-Body Theory Exact Solution. Phys. Rev. 1969, 178, 1097-1107.

367. Balasubramanian, T.; Andersen, J. N.; Walldén, L. Surface-Bulk Core-Level Splitting in Graphite. Phys. Rev. B 2001, 64, 205420.

368. Curve Fitting Functions. www.originlab.com/doc/Origin-Help/Curve-Fitting-Function (accessed September 3, 2015).

369. Blyth, R. I. R.; Buqa, H.; Netzer, F. P.; Ramsey, M. G.; Besenhard, J. O.; Golob, P.; Winter, M. XPS Studies of Graphite Electrode Materials for Lithium Ion Batteries. Appl. Surf. Sci. 2000, 167, 99-106.

370. van Attekum, P. M. T. M.; Wertheim, G. K. Excitonic Effects in Core-Hole Screening. Phys. Rev. Lett. 1979, 43, 1896-1898.

371. Cumpson, P. J.; Seah, M. P. Elastic Scattering Corrections in AES and XPS. II. Estimating Attenuation Lengths and Conditions Required for Their Valid Use in Overlayer/Substrate Experiments. Surf. Interface Anal. 1997, 25, 430-446. 